UNIVERSIDADE DE BRASÍLIA

FACULDADE DE TECNOLOGIA

DEPARTAMENTO DE ENGENHARIA MECÂNICA

Formulações do Método dos Elementos de Contorno para Análise de Placas Espessas Isotrópicas e Ortotrópicas

Autor: André Pereira Santana Orientador: Prof. Dr. Éder Lima de Albuquerque

TESE DE DOUTORADO EM CIÊNCIAS MECÂNICAS

PUBLICAÇÃO: ENM.TD-23/14

BRASÍLIA/DF: DEZEMBRO 2014 


\section{UNIVERSIDADE DE BRASÍLIA \\ FACULDADE DE TECNOLOGIA \\ DEPARTAMENTO DE ENGENHARIA MECÂNICA}

Tese de Doutorado

\section{Formulações do Método dos Elementos - de Contorno para Análise de Placas Espessas Isotrópicas e Ortotrópicas}

Autor: André Pereira Santana

Orientador: Prof. Dr. Éder Lima de Albuquerque

A Banca Examinadora composta pelos membros abaixo aprovou esta Tese:

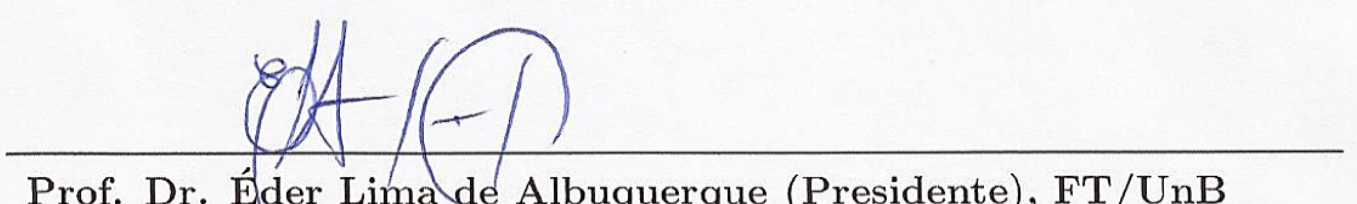

Prof. Dr. Éder Limalde Albuquerque (Presidente), FT/UnB

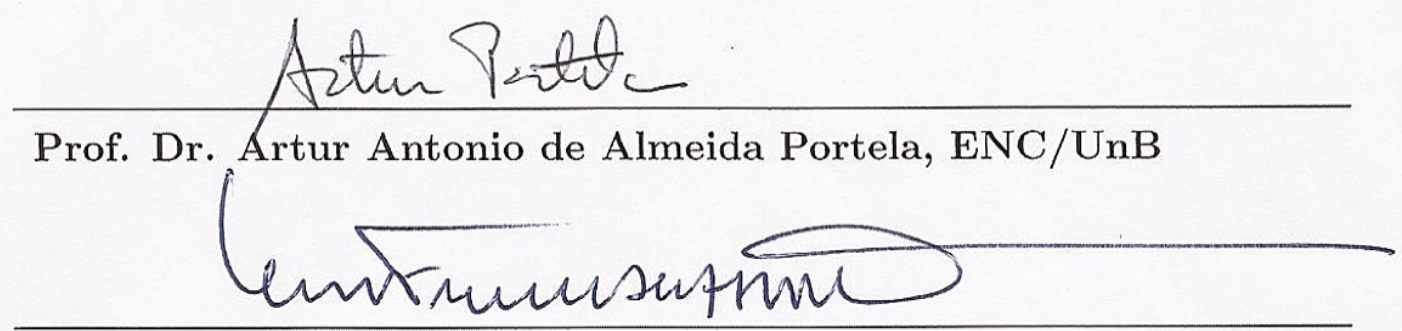

Prof. Dr. Carlos Friedrich Loeffler Neto, CT/UFES

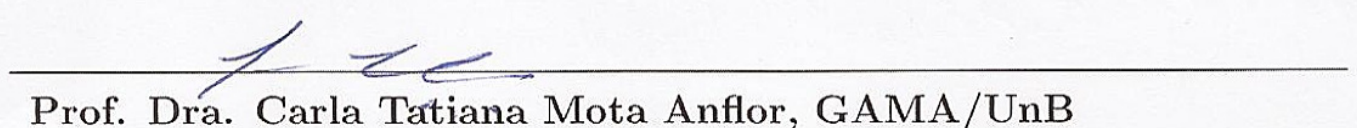

Prof. Dra. Carla Tátiana Mota Anflor, GAMA/UnB

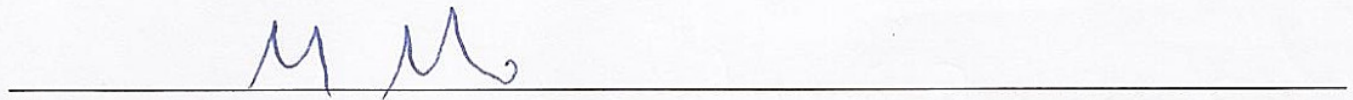

Prof. Dr. Paulo Sollero, FEM/UNICAMP 
Ficha catalográfica elaborada pela Biblioteca Central da Universidade de Brasília. Acervo 1019221.

Santana, André Pereira.

A345f Formulações do método dos elementos de contorno para anál ise de placas espessas isotrópicas e ortotrópicas / André Perei ra Santana. - 2014.

xxviii, 202 p. : il. ; $30 \mathrm{~cm}$.

Tese (doutorado) - Universidade de Brasília, Faculdade de Tecnologia, Pós-Graduação em Ciências Mecânicas, 2014. Or ientação: Éder Lima de Albuquerque. Inclui bibliografia.

1. Materiais compostos. 2. Métodos de elementos de contorno. I. Albuquerque, Éder Lima de. orient. II. Título. 


\author{
UNIVERSIDADE DE BRASÍLIA \\ FACULDADE DE TECNOLOGIA \\ DEPARTAMENTO DE ENGENHARIA MECÂNICA
}

\title{
Formulações do Método dos Elementos de Contorno para Análise de Placas Espessas Isotrópicas e Ortotrópicas
}

Autor: André Pereira Santana

Orientador: Prof. Dr. Éder Lima de Albuquerque

Curso: Engenharia Mecânica

Área de concentração: Ciências Mecânicas

Tese de Doutorado apresentada ao curso de Doutorado em Ciências Mecânicas da Faculdade de Tecnologia da Universidade de Brasília, como requisito para a obtenção do título de Doutor em Ciências Mecânicas.

Brasília, 2014

DF - Brasil 


\section{Dedicatória}

- Aos meus pais e irmãos que apesar das adversidades financeiras sempre incentivaram meus estudos. Obrigado por tudo! 


\section{Agradecimentos}

- Inicialmente, a Deus, pela saúde, pelo crescimento e por fim, pela missão cumprida.

- Ao meu orientador, Prof. Éder Lima de Albuquerque, pela extraordinária orientação, comprometimento, apoio, paciência, por tantos conhecimentos transmitidos, pela amizade e estímulo constante para o bom decorrer do trabalho.

- Aos professores do departamento de mecânica e materiais do IFMA por terem sempre me propiciado as melhores condições para realizar este trabalho (Carmem, Castro, Ernandes, Junior, Jean, Juca, Keyll, Mauro, Milton, Politi, Tiago (in memorian) e Waldemir).

- Ao Prof. Ferdinando pela amizade conquistada nos últimos anos.

- Aos meus primeiros alunos do IFMA: Daniel Coimbra, Kadu, Nathan Freitas, Raul Everton e Vitor Cordeiro.

- Aos alunos de iniciação cientifica e monografia: Alisson Augusto, Helio Vitor, Kamyla Castro, Samuel Santos (in memorian), Francisco de Assis, Newton Mesquita, Clarianne Natali, Vitor Cordeiro e Eric Maia. Obrigado pela confiança!

- Aos amigos Eric e Shirlen pela companhia saudável.

- A Clarianne Natali pela amizade e respeito.

- Ao amigo, Dalmo Galdez pela grande amizade cultivada desde a graduação!

- A Adriana Reis, Carlos André e Diogo Moraes, pela disposição de sempre.

- À Marilise, pela amizade conquistada e companhia agradável de sempre!

- Aos amigos Jairo Useche e Lucas Campos que sempre mostraram-se dispostos a colaborar neste trabalho.

- Ao Prof. Paulo Sollero, pela amizade, pelos ensinamentos passados durante o período de estágio docente na UNICAMP.

- Também gostaria de mencionar minha sincera admiração e gratidão aos professores Doval, Rubens e Politi, que fundamentaram minha base científica e muito contribuiram para o meu crescimento profissional.

- Aos professores Luciano Mendes Bezerra e Artur Portela pelas críticas construtivas durante a qualificação de doutorado.

- A Rodrigo Rocha pelo companheirismo. 
- Aos meus tios Salomão e Antonio que sempre impulsionaram meu crescimento profissional, fica aqui meu muito obrigado.

- Aos amigos de república, Adilto e Flavio, pela amizade.

- A todos que não foram citados aqui e que sempre acreditaram no meu trabalho e na minha vontade de transmitir conhecimento.

- A Universidade de Brasília pelas condições de trabalho.

- A FAPEMA pelo apoio financeiro. 
All we need to do is make sure we keep talking. (Trecho da música Keep Talking - Pink Floyd.) 


\section{Resumo}

Santana, André Pereira, Formulações do Método dos Elementos de Contorno para Análise de Placas Espessas Isotrópicas e Ortotrópicas. Brasília, 2014. Tese de Doutorado, Faculdade de Tecnologia, Universidade de Brasília.

Este trabalho apresenta formulações estáticas do método dos elementos de contorno para problemas de placas isotrópicas e ortotrópicas em flexão através da teoria de Reissner e Mindlin, respectivamente. A solução fundamental de placas espessas ortotrópicas que leva em conta o efeito do cisalhamento transversal são obtidas usando o operador de Hörmander e transformada de Radon. O operador de Hörmander é usado para transformar os sistemas de equações diferenciais parciais que representa as equações de equilíbrio em apenas uma equação diferencial parcial. Usando a transformada de Radon, essa equação diferencial parcial é reduzida a uma equação diferencial ordinária. Para obter a transformação inversa, integrais singulares precisam ser calculadas. Uma simples quadratura é usada onde integrais fortes e hipersingulares são tratadas no sentido de Cauchy e Hadamard, respectivamente. Derivadas da solução fundamental são usadas na equação integral de contorno para o cálculo de momentos em pontos internos. A formulação desenvolvida é aplicada no cálculo de deslocamentos, tensões e momentos em placas submetidas a cargas distribuída no domínio da estrutura. As integrais de superfície provenientes das cargas de domínio são transformadas em integrais de contorno usando o método da integração radial. Apenas o contorno é discretizado em todas as formulações implementadas e são utilizados elementos constantes (1 nó por elemento). Os resultados obtidos são comparados com resultados analíticos disponíveis na literatura, com o método sem malhas e com o método dos elementos finitos. Em geral há uma boa concordância entre os resultados obtidos neste trabalho com os resultados da literatura.

Palavras chaves: Métodos dos elementos de contorno, placas espessas, materiais compósitos. 


\begin{abstract}
Santana, André Pereira, Formulation of the boundary element method to the analysis of isotropic and orthotropic thick plates. Brazilia, 2014. PhD Thesis, Faculty of Technology, University of Brazilia.
\end{abstract}

This work presents a static formulation of the boundary element method for problems of isotropic and orthotropic plates at bending through Reissner and Mindlin's theory, respectively. The fundamental solution of the orthotropic shear deformable plates are obtained using Hörmander operator and Randon transform. The Hörmander operator is used to transform the partial differential equation system that represents the equilibrium equation in only one partial differential equation. Using Radon transform, this partial differential equation is reduced to an ordinary differetial equation. To obtain the inverse transformation, singular integrals need to be computed. A simple quadrature is used where strong and hypersingular integrals are treated in Cauchy and Hadamard sense, respectively. Derivatives of fundamental solutions are used in boundary integral equations to compute moments at internal points. The developed formulations are applied to compute displacements, stresses and moments at plates submitted to transversal loads in the structure domain. Surface integrals that come from domain loads are transformed into boundary integrals using the radial integration method. Only the boundary is discretized at all the implemented formulations and are used constants elements (1 node per element). Results are compared with analytical meshless and finite element results available in literature. In general there is a good agreement between the results obtained in this work and those available in literature.

Key words: Boundary element method, thick plates, composite materials. 


\section{Sumário}

1 Introdução $\quad 1$

1.1 Revisão bibliográfica . . . . . . . . . . . . . . . . . . . . . 1

1.2 Considerações sobre materiais compósitos . . . . . . . . . . . . . . . 3

1.3 Considerações sobre placas . . . . . . . . . . . . . . . . . 4

1.4 Evolução do método dos elementos de contorno . . . . . . . . . . . . . 5

1.5 Objetivos deste trabalho . . . . . . . . . . . . . . . 6

1.6 Descrição do presente trabalho . . . . . . . . . . . . . . 7

2 Materiais compósitos $\quad 10$

2.1 Introdução . . . . . . . . . . . . . . . . . . . . . . . . . 10

2.2 Compósitos . . . . . . . . . . . . . . . . . . . . 10

2.3 Componentes constituintes de um material compósito . . . . . . . . . . . . 11

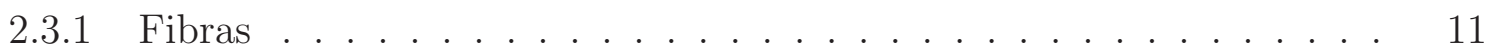

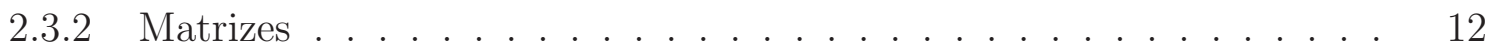

2.4 Interesse dos materiais compósitos . . . . . . . . . . . . . . . . . . 13 
2.5 Aplicações dos materiais compósitos . . . . . . . . . . . . . . . 13

2.6 Laminados . . . . . . . . . . . . . . . . . . . . . . . . . . . . . 15

2.7 Equação constitutiva do laminado . . . . . . . . . . . . . . . . . . . . . . . 17

3 Equações de elasticidade linear $\quad 20$

3.1 Introdução . . . . . . . . . . . . . . . . . . . . . . . . . . . 20

3.2 Deslocamentos e deformações . . . . . . . . . . . . . . . . . . 20

3.3 Tensões e equações de equilíbrio . . . . . . . . . . . . . . . . . . . . 24

3.4 Equação constitutiva . . . . . . . . . . . . . . . . . . . . . . . 26

3.5 Relações de tensão $\times$ deformação e deslocamento em placas . . . . . . . . . 27

3.6 Equações de equilíbrio em coordenadas cilíndricas . . . . . . . . . . . . . . . 29

4 Placas espessas isotrópicas $\quad 31$

4.1 Introdução . . . . . . . . . . . . . . . . . . . . . . . . 31

4.2 Teoria de Mindlin . . . . . . . . . . . . . . . . . . . . . . 31

4.3 Esforços generalizados e curvaturas . . . . . . . . . . . . . . . . . . 34

4.4 Trabalho virtual . . . . . . . . . . . . . . . . . . . . . 38

4.5 Equações de equilíbrio . . . . . . . . . . . . . . . . . . . . . . 42

4.6 Relações tensões $\times$ deslocamentos . . . . . . . . . . . . . . . . . . . . . 44

4.7 Condições de contorno . . . . . . . . . . . . . . . . . . . 47

4.7 .1 Borda apoiada . . . . . . . . . . . . . . 47

4.7 .2 Borda engastada . . . . . . . . . . . . . . 47 
4.7 .3 Borda livre . . . . . . . . . . . . . . . . . . 48

4.8 Avaliação das constantes . . . . . . . . . . . . . . . . . . . . 49

4.9 Equação de Navier . . . . . . . . . . . . . . . . . . . . . 50

5 Método dos elementos de contorno para placas isotrópicas 53

5.1 Introdução . . . . . . . . . . . . . . . . . . . . . . . . 53

5.2 Representação integral . . . . . . . . . . . . . . . . . . . . 53

5.3 Equação integral de contorno . . . . . . . . . . . . . . . . . . 59

5.4 Discretização . . . . . . . . . . . . . . . . . . . . . . . 60

5.4.1 Elementos constantes . . . . . . . . . . . . . . . . . . 60

5.5 Tratamento de singularidades . . . . . . . . . . . . . . 62

5.6 Sistema de equações $\ldots \ldots$. . . . . . . . . . . . . . . . . . . . . 63

5.7 Tensões em pontos internos . . . . . . . . . . . . . . . . 63

5.8 Equação integral hipersingular . . . . . . . . . . . . . . . . 65

5.8.1 Equação integral de flexão . . . . . . . . . . . . . . . . . 67

5.8.2 Equação integral da força cortante . . . . . . . . . . . . . . 71

5.9 Tensões no contorno f . . . . . . . . . . . . . . . . . . . . 75

5.10 Equação integral de tração . . . . . . . . . . . . . . . . . 75

6 Transformação das integrais de domínio em integrais de contorno $\quad 77$

6.1 Transformação exata . . . . . . . . . . . . . . . . . . 77 
7.1 Resultados . . . . . . . . . . . . . . . . . . . . . . . 81

7.1.1 Placa quadrada apoiada nos quatro lados sob carga uniformemente distribuída . . . . . . . . . . . . . . . . . . 81

7.1.2 Placa quadrada engastada nos quatro lados sob carga uniformemente distribuída . . . . . . . . . . . . . . . . . 96

7.1.3 Placa quadrada com um lado engastado e três lados apoiados sob carga uniformemente distribuída . . . . . . . . . . . . . . 103

8 Placas espessas ortotrópicas

8.1 Introdução . . . . . . . . . . . . . . . . . . . . . . . . . . . . . 108

8.2 Equações básicas . . . . . . . . . . . . . . . . . . . . . . 108

8.3 Equação diferencial de equilíbrio . . . . . . . . . . . . . . . . . 110

8.4 Relação entre forças generalizadas $\left(M_{\alpha \beta}\right.$ e $\left.Q_{\alpha}\right)$ e deslocamentos $(\Psi$ e $W)$. . 111

8.5 Forças e deslocamentos no contorno $\Gamma \ldots \ldots \ldots \ldots$. . . . . . . . . 112

8.6 Solução fundamental . . . . . . . . . . . . . . . . . . . . . . . . . . . . . . . 112

8.6.1 Cálculo da solução fundamental . . . . . . . . . . . . . . . . . . 121

8.7 Tratamento numérico da solução fundamental . . . . . . . . . . . . . . . 123

8.7.1 Solução fundamental $U_{i j}^{*} \ldots \ldots \ldots$. . . . . . . . . . . 123

8.7 .2 Solução fundamental $P_{i j}^{*} \ldots \ldots \ldots$. . . . . . . . . . . . 129

8.8 Equações integrais de contorno . . . . . . . . . . . . . . . . . . . . 133

8.8.1 Derivadas da solução fundamental $U_{i j}^{*}$ e $P_{i j}^{*} \ldots \ldots$. . . . . . . . . . 134 
8.8.2 Tratamento de singularidades . . . . . . . . . . . . . 137

8.8.3 Validação dos resultados . . . . . . . . . . . . . . . . . 138

9 Resultados para placas ortotrópicas $\quad 140$

9.1 Resultados . . . . . . . . . . . . . . . . . . . . . 140

9.1.1 Placa quadrada apoiada nos quatro lados sob carga uniformemente distribuída . . . . . . . . . . . . . . . . . . . 141

9.1.2 Placa quadrada engastada nos quatro lados sob carga uniformemente distribuída . . . . . . . . . . . . . . . . . . . 149

9.1.3 Placa quadrada com um lado engastado e os demais livres . . . . . . 156

10 Conclusões $\quad 159$

10.1 Considerações finais . . . . . . . . . . . . . . . . . . . . 159

10.2 Trabalhos Futuros . . . . . . . . . . . . . . . . 160

A Método de Hörmander 172

A.1 Exemplo do uso do operador de Hörmander . . . . . . . . . . . . . . . 173

$\begin{array}{lr}\text { B Transformada de Radon } & 176\end{array}$

B.1 Definição da transformada de Radon . . . . . . . . . . . . . . . . . 177

B.2 Exemplo do uso da transformada de Radon . . . . . . . . . . . . . . . 178

$\begin{array}{ll}\text { C Método das diferenças finitas } & 181\end{array}$

$\begin{array}{lr}\text { D Funções singulares } & 182\end{array}$ 
$\begin{array}{lll}\text { F } & \text { Quadratura para integrais singulares } & 187\end{array}$

F.1 Definição . . . . . . . . . . . . . . . . . . . . . . . . . . . 187

F.2 Quadratura proposta .......................... 189

G Desenvolvimento da derivada da solução fundamental 191

H Desenvolvimento dos kernels da solução fundamental $U_{i j}^{*} \quad 196$

H.1 Desenvolvimento do kernel $\tilde{U}_{\alpha \beta} \quad \ldots \ldots \ldots$. . . . . . . . . . . . . . . . 198

H.2 Desenvolvimento dos kernels $\tilde{U}_{3 \alpha}=-\tilde{U}_{\alpha 3} \ldots \ldots \ldots \ldots$. . . . . . . . . 200

H.3 Desenvolvimento do kernel $\tilde{U}_{33} \ldots \ldots \ldots \ldots$. . . . . . . . . . . . . . 201 


\section{Símbolos}

\section{Letras gregas}

$\alpha, \beta=$ Constantes.

$\varepsilon=$ Deformação.

$\bar{\varepsilon}=$ Deformação virtual.

$\chi=$ Curvatura.

$\phi=$ Rotação.

$\Gamma=$ Contorno.

$\gamma=$ Deformação cisalhante.

$\mu=$ Raíz do polinômio característico.

$\nu=$ Razão de Poisson.

$\Omega=$ Domínio.

$\theta=$ Rotação.

$\bar{\theta}=$ Rotação virtual.

$\rho=$ Distância entre pontos.

$\sigma=$ Tensão normal.

$\tau=$ Tensão cisalhante.

$\Pi=$ Energia.

$\psi=$ Deformação por cisalhamento.

$\chi=$ Deformação por flexão.

$\lambda=$ Fator de correção.

$\nabla=$ Operador laplaciano. 
$\Delta=$ Operador diferencial.

$\zeta, \xi, \eta=$ Ponto campo.

$\Phi=$ Função.

$\mu=$ Constantes.

$\partial=$ Derivada parcial.

$\varphi=$ Função.

$\pi=$ pi.

$\varphi, \psi=$ Funções suaves.

$\psi=$ Solução fundamental.

$\Re=$ Real.

\section{Letras arábicas}

$A=$ Área .

$a, b, c=$ Constantes.

$b=$ Força de corpo generalizada.

$h=$ Espessura .

$C, K=$ Constantes.

$D=$ Rigidez a flexão.

$\mathbf{C}=$ Tensor de quarta ordem.

$\mathbf{D}=$ Matriz de rigidez de flexão.

$\mathbf{D}^{\prime}=$ Matriz $\mathbf{D}$ transformada.

$d_{i}=$ Parte real de $\mu$.

$E=$ Módulo de elasticidade.

$G=$ Módulo de cisalhamento.

$e_{i}=$ Parte imaginária de $\mu$.

$p=$ Forças de superfície generalizadas.

$M, m=$ Momentos.

$N=$ Função de interpolação.

$\mathbf{n}=$ Vetor normal ao contorno. 
$p=$ Força de superfície.

$Q=$ Força cortante.

$\mathrm{Q}=$ Matriz de rigidez.

$\overline{\mathbf{Q}}=$ Matriz de rigidez transformada.

$q=$ Força distribuída.

$R_{i}=$ Função.

$r_{i}, s_{i}, q_{i}, p_{i}=$ Constantes.

$S_{i}=$ Função.

$\mathbf{T}=$ Matriz de transformação.

$t=$ Forças de superfície.

$u=$ Deslocamento no plano.

$w=$ Deslocamento transversal.

$\bar{\omega}=$ Deslocamento transversal virtual.

$z=$ Distância transversal do plano médio à um ponto.

$W=$ Esforços externos.

$T=$ Esforços internos.

$F=$ Forças de volume.

$L=$ Operador diferencial.

$I=$ Integral.

$K=$ Constantes de Bessel.

$V=$ Volume.

$x, y=$ Ponto campo.

$X=$ Ponto campo.

$X^{\prime}=$ Ponto interno.

$A, B=$ Funções de Bessel.

$D=$ Módulo de rigidez.

$U, P, V, W=$ Kernels.

$H, G=$ Matrizes.

$s, f=$ Funções.

$d, g=$ Funções 


\section{Subscritos}

$\Gamma=$ Contorno.

$\Omega=$ Domínio.

1, 2, $3=$ Direções principais.

$f=$ Fibra.

$L=$ Direção longitudinal às fibras.

$m=$ Matriz

$n=$ Direção normal.

$s=$ Direção tangencial.

$T=$ Direção transversal às fibras.

$t=$ Tração.

$x_{1}, x_{2}, x_{3}=$ Eixos do sistema de coordenadas.

$u_{1}, u_{2}, u_{3}=$ Eixos do sistema de coordenadas.

$u_{r}, u_{\theta}, w=$ Eixos de coordenadas cilíndricas.

$\alpha, \beta, \gamma=$ Índices gregos.

$i, j, k=$ Índices latinos.

\section{Sobrescritos}

1, 2, 3 = Nós do elemento.

$*=$ Soluções fundamentais.

. = Primeira derivada.

.. = Segunda derivada. 


\section{Abreviações}

$e x p=$ Exponencial.

$\log =$ Logaritmo.

ref $=$ Referência.

num = Numérico.

dif $=$ Diferença.

$M E C=$ Método dos Elementos de Contorno.

$M E F=$ Método dos Elementos Finitos.

$M D F=$ Método das Diferenças Finitas.

$C D C=$ Condição de Contorno.

$V P C=$ Valor principal de Cauchy.

$V P H=$ Valor principal de Hadamard.

$c o=$ Cofator .

$\sin =$ Seno.

$\cos =$ Cosseno.

tan $=$ Tangente.

$l i m=$ Limite.

$a d j=$ Adjunto . 


\section{Lista de Figuras}

2.1 Compósitos com reforço tipo: a) unidirecional; b) tecido bidirecional; c) fibras picadas; e d) manta contínua. . . . . . . . . . . . . . . . 11

2.2 Boeing 787 - Principais aplicações estruturais em materiais compósitos. Crédito: The Boeing Company. . . . . . . . . . . . . . . . . . 14

2.3 Mercedes-Benz SLR McLaren - Chassi construído em fibra de carbono. Crédito: Portal Mercedes-Benz Brasil. . . . . . . . . . . . . . . . . 15

2.4 Lâmina compósita reforçada com fibras longas unidirecionais. . . . . . . . . . 16

2.5 Ângulo entre fibras unidirecionais e sistema de referência. . . . . . . . . . . . 18

3.1 Estado de deformação de um sólido. . . . . . . . . . . . . . . . . . . 21

3.2 Forças de superfície e volume. . . . . . . . . . . . . . . . . . 25

3.3 Sistema de coordenadas cilíndricas. . . . . . . . . . . . . . . 28

3.4 Elemento infinitesimal. . . . . . . . . . . . . . . . . . . 29

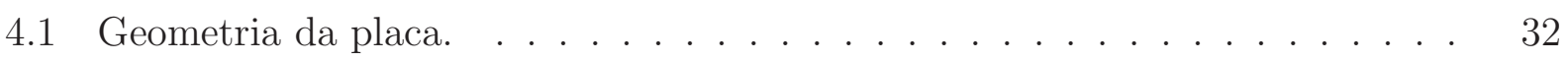

4.2 Deslocamentos generalizados. . . . . . . . . . . . . . . . . 33

4.3 Distribuição de tensões ao longo da espessura. . . . . . . . . . . . . . . . 35 
4.4 Convenção de sinal para forças de superfície generalizadas em pontos do contorno.

4.5 Rotações no contorno. . . . . . . . . . . . . . . . . . . . . . . 40

4.6 Equilíbrio em um elemento diferencial de placa. . . . . . . . . . . . . . . 42

4.7 Placa com borda apoiada. . . . . . . . . . . . . . . . . . 48

4.8 Placa com borda engastada. . . . . . . . . . . . . . . . . . . . . 48

5.1 Domínio bidimensional dividido em elementos de contorno. . . . . . . . . . . 61

5.2 Deslocamento e tração no contorno. . . . . . . . . . . . . . . . . . . . . . 61

5.3 Região semicircular em torno do ponto fonte. . . . . . . . . . . . . . 66

6.1 Transformação da integral de domínio em integral de contorno. . . . . . . . .

7.1 Placa quadrada apoiada nos quatro lados. . . . . . . . . . . . . . . 82

7.2 Discretização com 40 elementos no contorno e 1 nó interno. . . . . . . . . . . 83

7.3 Discretização com 40 elementos no contorno e 19 nos internos. . . . . . . . . 92

7.4 Deslocamento vertical ao longo da linha central da placa. . . . . . . . . . 93

7.5 Momentos na direção $x$ ao longo da linha central da placa. . . . . . . . . . . 93

7.6 Momentos na direção y ao longo da linha central da placa. . . . . . . . . . . 94

7.7 Mapa de cor do deslocamento vertical do nó central da placa de espessura

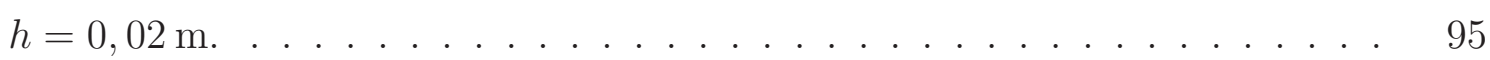

7.8 Mapa de cor do momento $\left(m_{x x}\right)$ da placa de espessura $h=0,02 \mathrm{~m} . \quad \ldots \quad 95$

7.9 Placa quadrada engastada nos quatro lados. . . . . . . . . . . . . . . . 96 
7.10 Deslocamentos na direção $w$ ao longo da linha central da placa.

7.11 Momentos na direção $x$ ao longo da linha central da placa. . . . . . . . . . .

7.12 Momentos na direção y ao longo da linha central da placa.

7.13 Momentos na direção $x$ ao longo do lado $A B$ da placa.

100

7.14 Momentos na direção $y$ ao longo do lado $A B$ da placa.

7.15 Mapa de cor do deslocamento vertical do nó central da placa de espessura $h=0,02 \mathrm{~m}$.

7.16 Mapa de cor do momento $\left(m_{x x}\right)$ da placa de espessura $h=0,02 \mathrm{~m}$.

7.17 Placa quadrada com um lado engastado e os outros três apoiados.

7.18 Mapa de cor do deslocamento vertical do nó central da placa de espessura $h=0,02 \mathrm{~m}$.

7.19 Mapa de cor do momento $\left(m_{x x}\right)$ da placa de espessura $h=0,02 \mathrm{~m}$. 107

8.1 Kernel $\tilde{U}_{11}$. 124

8.2 Kernel $\tilde{U}_{12}$.

8.3 Kernel $\tilde{U}_{21}$.

8.4 Kernel $\tilde{U}_{22}$.

8.5 Kernel $\tilde{U}_{31}$.

8.6 Kernel $\tilde{U}_{32}$.

8.7 Kernel $\tilde{U}_{13}$.

8.8 Kernel $\tilde{U}_{23}$. 127

8.9 Kernel $\tilde{U}_{33}$. 
8.10 Kernel $\tilde{P}_{11}$. . . . . . . . . . . . . . . . . . . . . . . . . . 129

8.11 Kernel $\tilde{P}_{12} \ldots \ldots \ldots \ldots \ldots \ldots \ldots \ldots \ldots$

8.12 Kernel $\tilde{P}_{21} \ldots \ldots \ldots \ldots \ldots \ldots \ldots \ldots$

8.13 Kernel $\tilde{P}_{22} \ldots \ldots \ldots \ldots \ldots \ldots \ldots \ldots$

8.14 Kernel $\tilde{P}_{31} \ldots \ldots \ldots \ldots \ldots \ldots \ldots \ldots \ldots$

8.15 Kernel $\tilde{P}_{32} \ldots \ldots \ldots \ldots \ldots \ldots \ldots \ldots \ldots \ldots$

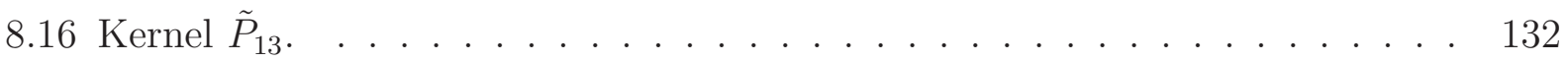

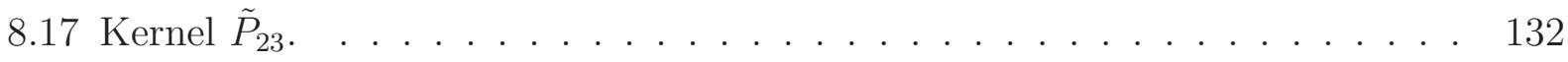

8.18 Kernel $\tilde{P}_{33} \ldots \ldots \ldots \ldots \ldots \ldots \ldots \ldots \ldots \ldots$

9.1 Placa quadrada apoiada nos quatro lados. . . . . . . . . . . . . . . 141

9.2 Discretização com 48 elementos no contorno e 1 nó interno. . . . . . . . . . . . 142

9.3 Discretização com 32 elementos no contorno e 15 nós internos. . . . . . . . . 143

9.4 Variação do deslocamento transversal $w$ devido um carregamento estático uniformemente distribuído. . . . . . . . . . . . . . . . . . 144

9.5 Variação do momento $m_{x x}$ devido um carregamento estático uniformemente distribuído. . . . . . . . . . . . . . . . . . 145

9.6 Variação da tensão $\sigma_{x x}$ devido um carregamento estático uniformemente distribuído. . . . . . . . . . . . . . . . . . . . . 145

9.7 Mapa de cor do deslocamento vertical do nó central da placa de espessura $h=0,0254 \mathrm{~m} \ldots \ldots \ldots \ldots \ldots \ldots \ldots \ldots \ldots$

9.8 Mapa de cor do momento $\left(m_{x x}\right)$ da placa de espessura $h=0,0254 \mathrm{~m} . \quad \ldots \quad 148$ 
9.9 Placa quadrada engastada nos quatro lados. . . . . . . . . . . . . . . . 149

9.10 Variação do deslocamento transversal $w$ devido um carregamento estático uniformemente distribuído. . . . . . . . . . . . . . . . . . . 150

9.11 Variação do momento $m_{x x}$ devido um carregamento estático uniformemente distribuído.

9.12 Variação da tensão $\sigma_{x x}$ devido um carregamento estático uniformemente distribuído.

9.13 Momentos na direção $x$ ao longo do lado $A B$ da placa.

9.14 Momentos na direção y ao longo do lado $A B$ da placa.

9.15 Mapa de cor do deslocamento vertical do nó central da placa de espessura $h=0,0254 \mathrm{~m}$

9.16 Mapa de cor do momento $\left(m_{x x}\right)$ da placa de espessura $h=0,0254 \mathrm{~m}$.

9.17 Placa quadrada com um lado engastado e três lados livres.

9.18 Deslocamento vertical ao longo da linha central da placa. . . . . . . . . . . 157

9.19 Momento $\left(m_{x x}\right)$ ao longo da linha central da placa. . . . . . . . . . . . 157

9.20 Tensões $\left(\sigma_{x x}\right)$ ao longo da linha central da placa. . . . . . . . . . . . . . . 158

B.1 Transformada de Radon para o caso tri-dimensional: integração no plano $\alpha=$ $z_{i} x_{i}$ e trasformada da Inversa: integração sobre uma esfera unitária $z_{i} z_{i}=1$.

B.2 Coordenadas esféricas $z_{1}, z_{2}$ e $z_{3} \ldots \ldots \ldots \ldots \ldots \ldots$

E.1 Pontos de Gauss sem o uso da transformada de Telles (regular) e com seu uso. 186

F.1 Gráfico da função $1 / x \ldots \ldots$. . . . . . . . . . . . . . . . . . . . . 190 


\section{Lista de Tabelas}

7.1 Deslocamentos e esforços no centro de uma placa apoiada sujeita a um carregamento distribuído comparados com a condição soft de Palermo (2000). .

7.2 Deslocamentos e esforços no centro da placa para diferentes relações espessura/lado. . . . . . . . . . . . . . . . . . .

7.3 Deslocamentos e esforços no centro de uma placa apoiada sujeita a um carregamento distribuído comparados com a condição soft de Palermo (2000). .

7.4 Deslocamentos e esforços no centro da placa para diferentes relações espessura/lado. . . . . . . . . . . . . . . . . . . .

7.5 Deslocamentos e esforços no centro de uma placa apoiada sujeita a um carregamento distribuído: $h=0,02 \mathrm{~m}$ e $\nu=0,0$.

7.6 Deslocamentos e esforços no centro de uma placa apoiada sujeita a um carregamento distribuído: $h=0,02 \mathrm{~m}$ e $\nu=0,3$.

7.7 Deslocamentos e esforços no centro de uma placa apoiada sujeita a um carregamento distribuído: $h=0,2 \mathrm{~m}$ e $\nu=0,0 \ldots \ldots \ldots$

7.8 Deslocamentos e esforços no centro de uma placa apoiada sujeita a um carregamento distribuído: $h=0,2 \mathrm{~m}$ e $\nu=0,3 \ldots \ldots \ldots$

7.9 Momento no lado BC da placa apoiada. . . . . . . . . . . . . 
7.10 Deslocamentos e esforços no centro de uma placa engastada sujeita a um carregamento distribuído. . . . . . . . . . . . . . . . . . . 97

7.11 Deslocamentos e esforços no centro da placa para diferentes relações $h / a . \quad$. $\quad 97$

7.12 Esforços no lado BC da placa para diferentes relações $h / a . . .97$

7.13 Deslocamentos e esforços no centro de uma placa engastada sujeita a um car-

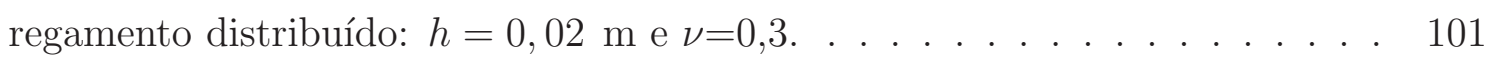

7.14 Deslocamentos e esforços no centro de uma placa engastada-apoiada sujeita a um carregamento distribuído: $h=0,02 \mathrm{~m}$ e $\nu=0,3 \ldots \ldots \ldots$. . . . . . . 104

7.15 Deslocamentos e esforços no centro de uma placa engastada-apoiada sujeita a

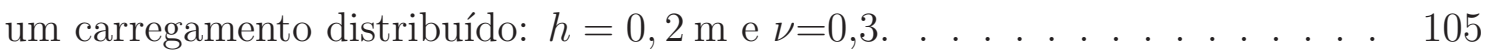

7.16 Deslocamentos e esforços ao longo do lado $C D$ da placa engastada-apoiada

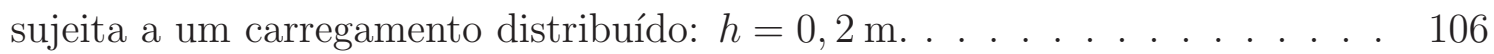

8.1 Matriz $P_{i j k}(:,:, 1)$ obtida através do MEC e MDF. . . . . . . . . . . 139

8.2 Matriz $P_{i j k}(:,:, 2)$ obtida através do MEC e MDF . . . . . . . . . . 139

8.3 Matriz $P_{i j k}(:,:, 3)$ obtida através do MEC e MDF. . . . . . . . . . . 139

9.1 Deslocamento transversal, momentos e tensões no centro da placa apoiada. . 143

9.2 Valores obtidos no centro da placa apoiada de espessura $h=0,0127 \mathrm{~m}$. . . 146

9.3 Momento no lado BC da placa apoiada. . . . . . . . . . . . . . . . . . 147

9.4 Deslocamento vertical e momentos no centro da placa engastada. . . . . . . . 150

9.5 Deslocamentos e esforços no centro da placa para diferentes relações $h / a$. . . 151

9.6 Valores obtidos no centro da placa engastada de espessura $h=0,0127 \mathrm{~m}$. . 152 
F.1 Solução para função $1 / x$ através da quadratura proposta por Campos e Albuquerque $(2013) \ldots \ldots \ldots \ldots \ldots \ldots$ 


\section{Capítulo 1}

\section{Introdução}

O presente trabalho trata de três assuntos: os materiais compósitos, as placas e o método dos elementos de contorno. A seguir são apresentadas as razões para a escolha desses três assuntos como objeto de estudo, bem como um pouco de sua história.

\subsection{Revisão bibliográfica}

Desde meados dos anos de 1800 tem-se desenvolvido modelos análiticos para representar o comportamento de placas. Os primeiros trabalhos foram desenvolvidos por Sophie Germain, Lagrange e Poisson (Pilkey e Wunderlich, 1994). As equações integrais ficaram conhecidas no século XIX e tiveram suas primeiras aplicações nos trabalhos de Betti (1872) e Somigliana (1885) com trabalhos voltados para elasticidade plana. A primeira formulação do método dos elementos de contorno baseado em equações integrais foi proposta por Kupradze (1965). Em seguida Rizzo (1967) propôs uma formulação para o MEC mais completa. Cruze (1969), Ricardella (1973) e Lachat (1973) introduziram funções de forma constante, linear e quadrático, respectivamente na formulação do MEC. Os trabalhos de Bezine (1978) e Stern (1979) foram as primeiras formulações do método dos elementos de contorno para placas finas utilizando as hipóteses de Kirchhoff (1950). 
Formulações de elementos de contorno para problemas anisotrópicos foram apresentados por Sollero e Aliabadi (1995). Portela et al. (1992a) apresentou para problemas estáticos a formulação de elementos de contorno dual. Aliabadi e Sollero (1998) apresentaram a formulação de elementos de contorno dual na análise de propagação de trincas em laminados ortotrópicos. Albuquerque et al. (1999) estendeu a formulação do método dos elementos de contorno dual para problemas elasto-dinâmicos em materiais anisotrópicos.

A formulação do método dos elementos de contorno para placas espessas tem atraído a atenção de muitos pesquisadores nos últimos anos. O primeiro trabalho sobre placas espessas de Reissner e Mindlin usando o MEC foi proposto por Weeën (1982). Barcellos e Westphal (1992) mostraram como transformar a solução fundamental de Reissner na solução fundamental de Kirchhoff, atribuindo zeros para partes do integrando que representam as deformações por cisalhamento. EL-Zafrany et al. (1994) apresentaram a derivação da equação integral de contorno e da solução fundamental para flexão de placas espessas de Reissner (1947). Palermo Jr. (2002) estudou a resposta harmônica de placas usando a solução no domínio da frequência para equação integral para hipóteses de Mindlin (1951).

Existem poucos trabalhos sobre o MEC para placas e cascas espessas ortotrópicas ou anisotrópicas que levem em conta o efeito do cisalhamento transversal. Wang e Huang (1991) apresentaram uma solução fundamental para placas espessas ortotrópicas considerando o efeito do cisalhamento transversal. Wang e Schweizerhof (1995) apresentaram uma formulação para análise estática de cascas anisotrópicas moderamente espessas. Em Wang e Schweizerhof (1996) essa formulação foi estendida para cascas ortotrópicas moderamente espessas.

O tratamento de funções singulares presentes na solução fundamental de placas finas e espessas é outro ponto importante na formulação do método dos elementos de contorno. Rajamoham e Raamachandran (1999) propuseram uma formulação onde as singularidades foram evitadas pela colocação do ponto fonte fora do domínio para evitar o tratamento de singularidades da solução fundamental em placas anisotrópicas. Rashed (1999) apresentou a derivação completa da equação hipersingular e demonstrou o procedimento para tratar a integrais hipersingulares para condições de contorno gerais de placas espessas isotrópicas. 
Wang e Huang (1991) apresentaram uma formulação do MEC para placas moderamente espessas ortotrópicas e usando a subtração de singularidade para tratar as singularidades nos kernels da solução fundamental. Marczak e Creus (2002) apresentaram o tratamento de singularidades em placas espessas através do uso da série de Laurent. Portela et al. (1992b), apresentaram a subtração de singularidades na formulação de elementos de contorno para placas trincadas. Paiva et al. (2003) apresentou um tratamento análitico para integrais singulares e hipersingulares da formulação de placas finas baseado na teoria de Kirchhoff.

\subsection{Considerações sobre materiais compósitos}

Inúmeras conquistas tecnológicas recentes, principalmente as relacionadas com as aplicações relevantes em áreas tais como aeronáutica, aeroespacial, petroquímica, naval, bioengenharia, automobilística, construção civil, de artigos esportivos, entre outras, somente se tornaram viáveis após o advento dos materiais compósitos estruturais ou simplesmente compósitos. Esta classe de materiais é bastante ampla e abrangente, compreendendo desde os polímeros reforçados com fibras, os materiais híbridos metal/compósito, os concretos estruturais e outros compósitos que incorporam matriz metálica ou cerâmica.

Embora a associação do termo compósitos esteja ligada às chamadas tecnologias de ponta, nas quais peças e dispositivos oriundos desse material são empregados em componentes utilizados em satélites, aeronaves e helicópteros, implantes ortopédicos e odontológicos, veículos de Fórmula 1, plataformas marítimas de petróleo, pontes, telescópios, instrumentos músicais e estruturas inteligentes em geral, a origem desta importante classe de materiais remonta a milhões de anos, uma vez que as madeiras, os ossos e os tecidos musculares são exemplos notáveis em termos de eficiência estrutural dos chamados compósitos naturais.

As pesquisas por materiais de alta resistência, alta rigidez e baixo peso impuseram ciclos históricos na aplicação de materiais na indústria aeronáutica. Partindo do uso da madeira, passando pelas ligas de alumínio e magnésio, chega-se ao estado atual, no qual a indústria aeroespacial está, cada vez mais, substituindo o uso de metais pelo uso de compósitos. 
A princípio com utilização restrita à aeroespacial, atualmente a utilização dos materiais compósitos modernos vem se estendendo aos mais diversos ramos industriais. O motivo desse crescimento é que esses materiais apresentam características bastante desejavéis para muitas aplicações em engenharia. Devido a sua grande importância, os materiais compósitos têm sido objeto de muitos estudos, com vários livros publicados sobre o assunto (Agarwal e Broutman, 1990, Gibson, 1994 e Hull e Clyne, 1996).

\subsection{Considerações sobre placas}

Placas, por definição, são elementos estruturais, simétricos em relação a um plano médio, cuja dimensão menor, que está na direção normal a este plano, é denominada espessura da placa. O carregamento, normalmente, é transversal ao plano médio da placa. De acordo com o material do qual é constituída, as placas podem ser classificadas como: anisotrópicas (com propriedades diferentes em direções não perpendiculares), ortotrópicas (com propriedades diferentes em duas direções perpendiculares), ou isotrópicas (com propriedades iguais em qualquer direção). E de acordo com a espessura, as placas são classificadas como finas e espessas. A literatura recomenda, para metais, a formulação de placas finas para $1 / 80<t / a<1 / 5$ e espessas para $t / a>1 / 5$, onde $t$ é a espessura e $a$ é a aresta da placa quadrada (Timoshenko, 1959). Os modelos matemáticos para placas finas não levam em conta os efeitos da deformação por cisalhamento transversal enquanto que nos modelos de placas espessas estes efeitos são considerados. A adoção de hipóteses simplificadoras, visando analisar a placa como um elemento bidimensional, fez surgir diferentes teorias para verificar o comportamento geral desta superfície estrutural. Kirchhoff (1850) estabeleceu as hipóteses fundamentais da teoria de placas finas, deduzindo a expressão da energia potencial para uma placa inclinada e aplicando o princípio dos trabalhos virtuais para obter uma equação diferencial, onde a rigidez a flexão foi definida em termos do módulo de Young e coeficiente de Poisson. Adicionalmente, ele percebeu que as três condições de contorno naturais propostas por Poisson (1829) não eram compatíveis com a natureza de quarta ordem da equação diferencial obtida e mostrou que estas poderiam ser reduzidas a duas condições 
de contorno naturais. Esta teoria não leva em conta o efeito da deformação pelo esforço cortante, assumindo-se que retas normais ao plano médio da placa permanecem normais após a deformação. As hipóteses apresentadas por Kirchhoff resultaram em uma equação diferencial de quarta ordem, na qual o deslocamento é dado em função de duas coordenadas no plano médio da placa. Esta equação pode ser uma eficiente representação do comportamento de placas finas para pequenos deslocamentos, apresentando boa precisão de resultados para uma grande variedade de carregamentos e geometrias. Entretanto, a teoria desenvolvida por Kirchhoff não apresenta bons resultados quando são analisadas placas de maior espessura. Mindlin (1951) formulou uma teoria para analisar placas moderadamente espessas onde, assumindo-se que as distorções que ocorrem na espessura são constantes, as tensões são obtidas a partir de uma função imposta para as deformações ao longo da espessura. O sistema de equações diferenciais obtido é de sexta ordem e também satisfaz as três condições de contorno requeridas. Uma formulação similar a de Mindlin foi obtida por Reissner (1947) porém, ao invés de uma função para as deformações foi imposto uma função para as tensões ao longo da espessura. As formulações apresentadas por Kirchhoff e Mindlin podem ser consideradas como expressivas contribuições para o aprimoramento da teoria bidimensional de placas.

\subsection{Evolução do método dos elementos de contorno}

Atualmente a simulação computacional de fenômenos físicos de problemas de engenharia tem sido uma das principais ferramentas que auxiliam os engenheiros no desenvolvimento de projetos. Vários fenômenos físicos, nas diversas áreas do conhecimento, podem ser representadas por equações diferenciais parciais. Em casos práticos porém, dificilmente essas equações possuem soluções analíticas. Por isso, métodos numéricos são empregados para se obter uma solução aproximada das equações que governam o problema. Problemas envolvendo análises térmicas, análises de tensões, escoamento de fluidos e eletromagnetismo, são apenas alguns exemplos da vasta quantidade de problemas que podem ser modelados através do método dos elementos de contorno. A formulação de elementos de contorno possui como atrativo a possibilidade de reduzir o número de dimensões do problema, o que leva a um 
conjunto reduzido de equações e a uma quantidade menor de dados requeridos para a computação. Só para citar alguns pesquisadores e suas diferentes linhas de pesquisas dentro da formulação de elementos de contorno temos: Loeffler e Mansur (1986) proporam a formulação do MEC para vibrações livres de barras e membranas, Dias Júnior et al. (2013) abordaram a formação de cones de água em reservatórios de petroléo usando o MEC, Bezerra e Saigal (1993) proporam a formulação do MEC para detecção de falhas em problemas de elasticidade e Pouzada (1999), estudou a formulação do MEC para a análise de problemas de propagação de ondas eletromagnéticas. Estes problemas podem ser modelados sob as variadas condições de contorno com o objetivo de simular computacionalmente as condições reais de serviço de um determinado componente mecânico. Essa prática tem sido cada vez mais utilizada atualmente, pois proporciona uma significativa economia de tempo e recursos financeiros durante o desenvolvimento de projetos. Vários ensaios práticos e a construção de protótipos, que outrora eram indispensavéis, podem ser substituídos por uma simulação computacional adequada feita por um programa de análise numérica.

\subsection{Objetivos deste trabalho}

Usando o método dos elementos de contorno, os objetivos deste trabalho são:

- Apresentar a formulação de elementos de contorno para placas espessas isotrópicas e ortotrópicas considerando o efeito da deformação por cisalhamento na direção transversal;

- Apresentar o tratamento da integral de domínio presente na equação integral de contorno através do método da integração radial (MIR);

- Calcular deslocamentos, momentos e tensões em pontos internos e no contorno de placas espessas isotrópicas e ortotrópicas;

- Emprego da transformada de Hörmander e transformada de Radon;

- Emprego da quadratura de Gauss e Telles; 
- Emprego da quadratura de Campos e Albuquerque (2013).

As hipóteses adotadas são:

- Problemas estáticos;

- Regime elástico.

\subsection{Descrição do presente trabalho}

A motivação para o desenvolvimento de uma formulação de elementos de contorno para análise do comportamento mecânico dos materiais compósitos advém da crescente utilização desses materiais devido às suas excelentes propriedades mecânicas. O método dos elementos de contorno vem sendo desenvolvido há várias décadas e através dos anos se consolidando como uma ferramenta de análise computacional extremamente útil em várias áreas da engenharia. Entretanto, a análise de problemas de materiais anisotrópicos usando o método dos elementos de contorno ainda demanda muita pesquisa. A presente contribuição deste trabalho para literatura é o cálculo das tensões em pontos internos e no contorno de placas espessas ortotrópicas sujeitas a carregamentos transversalmente distribuídos ou concentrados usando a quadratura proposta por Campos e Albuquerque (2013). Este trabalho é uma continuação do trabalho de Reis et al. (2013), que obteve resultados para deslocamentos em placas espessas ortotrópicas usando a transformada de Telles (1987) no cálculo da solução fundamental. Os únicos trabalhos que calculam deslocamentos e esforços (momentos) em placas espessas ortotrópicas encontrados na literatura foi apresentado por Wang e Huang (1991) e Reis et al. (2013). Três tipos de singularidades estão presentes nos kernels da solução fundamental de placas espessas ortotrópicas, como mostrado em Reis et al. (2013): fraca, forte e hipersingular. É proposta uma quadratura numérica para tratamento de tais singularidades utilizando-se poucos pontos de integração (Campos e Albuquerque, 2013). A precisão dos resultados numéricos apresentados no presente trabalho é analisada pela comparação com resultados numéricos obtidos na literatura. Em toda formulação desenvolvida 
foram utilizados elementos constantes para placas isotrópicas e ortotrópicas afim de simplicar a formulação matemática do problema.

O presente trabalho é disposto de 10 capítulos.

No Capítulo 1 é apresentada uma revisão bibliográfica sobre placas, compósitos e o métodos dos elementos de contorno.

No Capítulo 2 é apresentada uma classificação dos compósitos quanto à sua forma e uma visão geral sobre suas características, tais como: composição, interesse e aplicações.

No Capítulo 3 são apresentadas algumas considerações sobre a teoria da elasticidade, bem como relações entre tensões e deformações.

No Capítulo 4 são apresentadas algumas considerações sobre placas espessas isotrópicas onde se leva em conta o efeito do cisalhamento transversal. Serão obtidas as equações de equilíbrio de placas.

No Capítulo 5 a formulação de elementos de contorno para placas isotrópicas é apresentada de forma detalhada. Será apresentada a obtenção da equação integral de contorno. Também será apresentada a obtenção da solução fundamental. São apresentados os tratamentos de singularidades de forma numérica e análitica. É apresentada a formulação para cálculo de tensões em pontos internos e do contorno.

No Capítulo 6 é apresentada uma formulação para o tratamento da integral de domínio na formulação de elementos de contorno. A transformação exata da integral de domínio proveniente da carga distribuída em integral de contorno é realizada utilizando o método da integração radial (MIR).

No Capítulo 7 são apresentados alguns resultados numéricos obtidos para validar as rotinas implementadas. Foram obtidos valores para deslocamentos, momentos, e tensões em pontos internos e no contorno de placas isotrópicas.

No Capítulo 8 são obtidas as equações de equilíbrio de placas ortotrópicas e a formulação de elementos de contorno para placas ortotrópicas é apresentada. É proposta uma formulação 
para o tratamento das singularidades forte e hipersingular.

No Capítulo 9 são apresentados alguns resultados numéricos obtidos para validar as rotinas implementadas. Foram obtidos valores para deslocamentos, momentos e tensões em pontos internos e no contorno de placas ortotrópicas.

Por fim, no Capítulo 10 são apresentadas as considerações finais e conclusões obtidas através dos resultados obtidos nesta tese. Também são citados temas do presente trabalho que ainda demandam pesquisa. 


\section{Capítulo 2}

\section{Materiais compósitos}

\subsection{Introdução}

Neste capítulo é feita uma revisão sobre materiais compósitos e sua aplicabilidade no ramo industrial. Também será feita uma pequena descrição da modelagem matemática dos materiais compósitos.

\subsection{Compósitos}

Um material compósito (ou composto) é formado pela união de dois materiais de naturezas diferentes, resultando em um material de performance superior àquela de seus componentes tomados separadamente. O material resultante é um arranjo de fibras, contínuas ou não, de um material resistente (reforço) que são impregnados em uma matriz de resistência mecânica inferior as fibras. Após décadas de uso restrito em alguns setores da indústria, como na área de míssies, foguetes e aeronaves de geometrias complexas, os compósitos poliméricos estruturais, também denominados compósitos, têm ampliado a sua utilização em diferentes setores da indústria moderna, com um crescimento de uso de 5\% ao ano (Rezende e Botelho, 2008). Atualmente, a utilização de estruturas de alto desempenho e com baixo peso tem sido 
buscada também nas indústrias automotiva, esportiva, de construção civil, entre outras.

\subsection{Componentes constituintes de um material compósito}

\subsubsection{Fibras}

A fibra é o elemento constituinte que confere ao material compósito suas características mecânicas: rigidez, resistência à ruptura, etc. As fibras podem ser curtas, medindo centímetros, que são injetadas no momento da moldagem da peça, ou longas, que são cortadas após a fabricação da peça. Os compósitos obtidos com fibras contínuas podem apresentar reforço unidirecional, reforço bidirecional (tecidos), fibras picadas ou manta contínua. No caso de reforços uni-direcional ou bi-direcional, o material é moldado em camadas de forma que, em cada camada do compósito, as fibras são contínuas e dotadas de direções preferenciais (ver Figura 2.1).

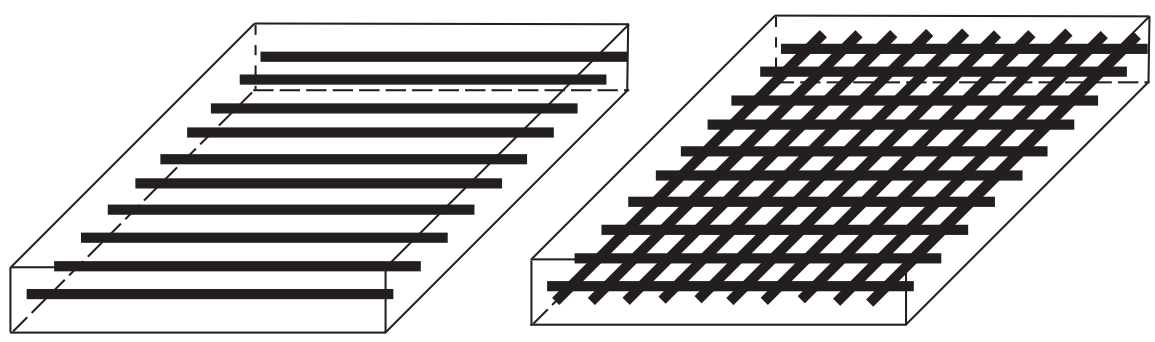

a) Unidirecional

b) Bidirecional

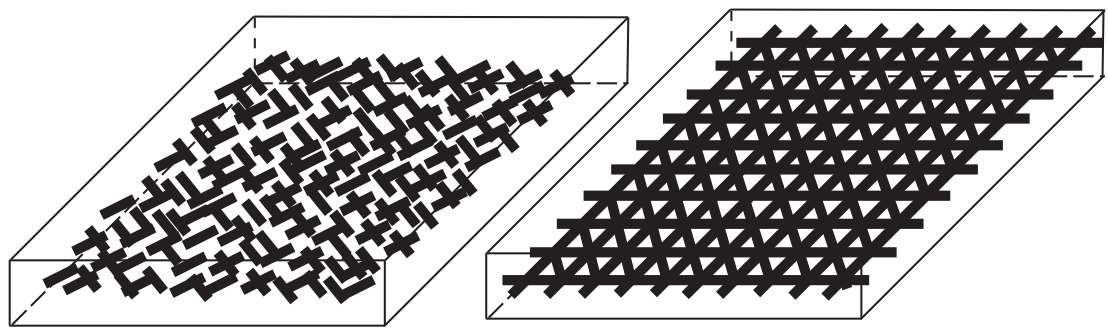

c) Fibras Picadas $\quad$ d) Manta Contínua

Figura 2.1: Compósitos com reforço tipo: a) unidirecional; b) tecido bidirecional; c) fibras picadas; e d) manta contínua. 
Se considerarmos que os compósitos esquematizados na Figura 2.1 são fabricados a partir de uma mesma matriz e de um tipo específico de fibra e submetidos a esforços de tração longitudinais, pode-se verificar diferenças em relação à eficiência de comportamento mecânico. Os compósitos obtidos a partir de lâminas com fibras unidirecionais e tecidos bidirecionais tendem a ser muito mais eficientes estruturalmente em relação aos compósitos obtidos com fibras picadas e mantas contínuas. No caso da Figura 2.1a, a resistência mecânica e a rigidez teriam maiores valores na direção longitudinal, em relação a Figura 2.1b, onde os resultados de resistência mecânica e rigidez apresentariam valores intermediários. Nas Figuras 2.1c e 2.1d, os valores de resistência mecânica e rigidez seriam menores que nas situações anteriores. Entretanto, tal fato só se verifica para esforços mecânicos longitudinais. Se os esforços fossem aplicados transversalmente, o melhor desempenho ocorreria na Figura 2.1b, e o pior desempenho, na Figura 2.1a. Estas tendências indicam que a orientação das fibras em relação aos esforços aplicados, considerando-se o fato de serem contínuas ou não, influênciam significativamente nas propriedades mecânicas dos compósitos. Os tipos mais comuns de fibras são: de vidro, de aramida (kevlar), carbono e boro.

\subsubsection{Matrizes}

As matrizes tem como função principal transferir as solicitações mecânicas às fibras e protegê-las do ambiente externo. As matrizes podem ser resinosas (polyester, epóxi, etc), minerais (carbono) e metálicas (ligas de alumínio). A escolha entre um tipo de fibra e uma matriz depende fundamentalmente da aplicação ao qual será dado o material compósito: características mecânicas elevadas, resistência à alta temperatura, resistência à corrosão, etc. O custo em muitos casos pode também ser um fator de escolha entre um ou outro componente. Deve ser observada também a compatibilidade entre as fibras e as matrizes. 


\subsection{Interesse dos materiais compósitos}

O interesse dos materiais compósitos está ligado a dois fatores: econômico e desempenho. O fator econômico vem do fato do material composto ser muito mais leve que os materiais metálicos, o que implica numa economia de combustível e consequentemente, num aumento de carga útil (vantagens especiais nas áreas aeronáutica e aeroespacial). A redução na massa total do produto pode chegar a $30 \%$ ou mais, em função da aplicação dada ao material compósito. O custo de fabricação de algumas peças em material compósito pode ser também sensívelmente menor se comparado com os materiais metálicos. O fator performance está ligado à procura por um melhor desempenho de componentes estruturais, sobretudo no que diz respeito as características mecânicas (resistência a ruptura, resistência à ambientes agressivos, etc.). O caráter anisotrópico dos materiais compósitos é o fator primordial para a obtenção das propriedades mecânicas requeridas pelo componente. A leveza, juntamente com as excelentes características mecânicas, faz com que os materiais compósitos sejam cada vez mais utilizados dentro de atividades esportivas.

\subsection{Aplicações dos materiais compósitos}

A aplicação dos materiais compósitos surgiu inicialmente na área aeronáutica devido à necessidade de estruturas de alto desempenho e baixo peso. Atualmente, uma grande variedade de peças em materiais compósitos pode ser encontrada nos aviões em substituição aos materiais metálicos: fuselagem, spoilers, portas de trem de aterrissagem, portas internas, etc. (ver Figura 2.2).

Em muitos destes componentes, sua concepção foge da definição dada inicialmente para materiais compósitos, pois nestes casos os componentes são fabricados normalmente por placas de baixa densidade, contra-placadas por placas finas de alta resistência. Esta configuração normalmente é dita sanduíche. De uma forma mais ampla, estas configurações são também consideradas materiais compósitos, pois combinam diferentes materiais. Dentro da área aeronáutica, os helicópteros possuem também vários componentes em material compósito: pás 


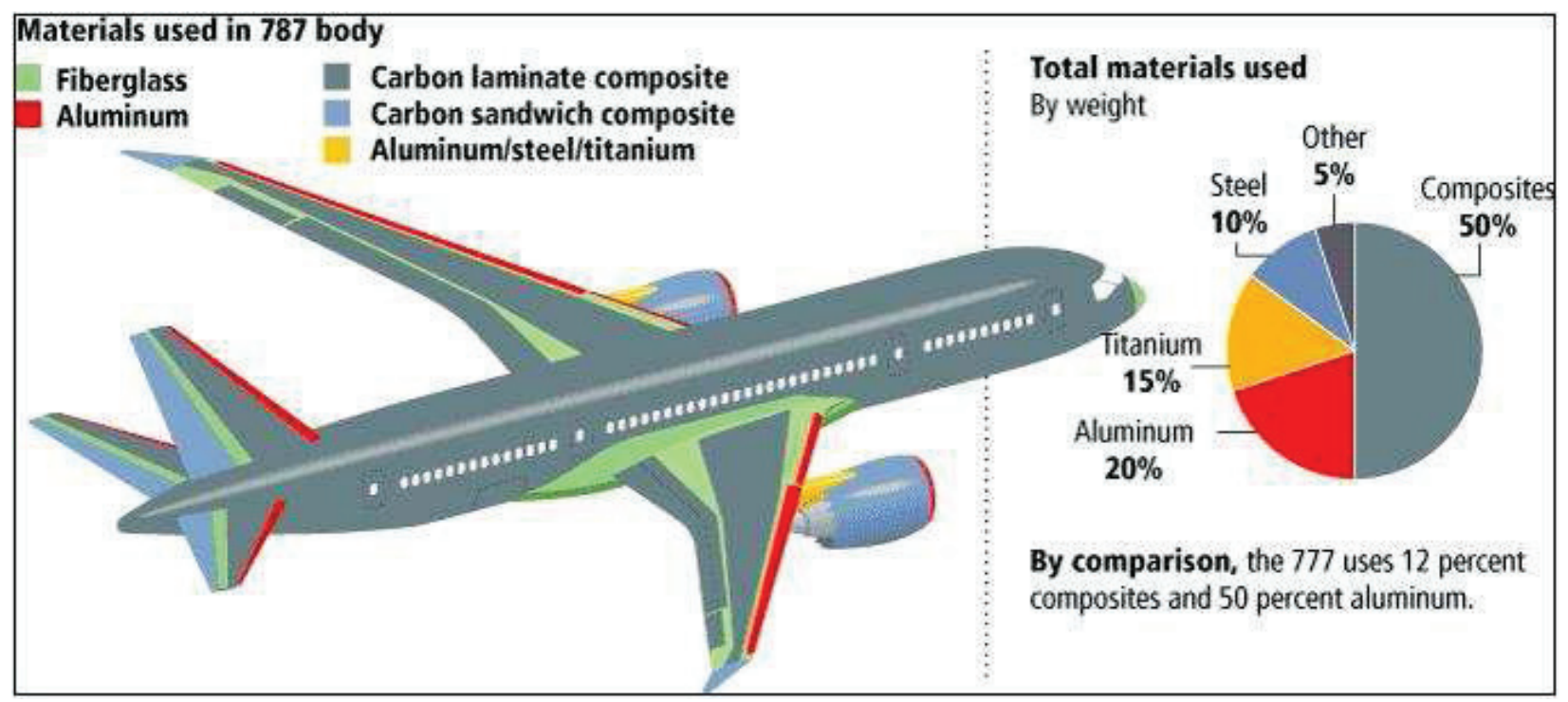

Figura 2.2: Boeing 787 - Principais aplicações estruturais em materiais compósitos. Crédito: The Boeing Company.

da hélice principal, hélice traseira, árvore de transmissão, fuselagem, etc.

A utilização dos materiais compósitos dentro da indústria automobilística é bem mais recente do que na área aeronáutica. Inicialmente, eram produzidos somente parachoques e tetos de automóveis. Atualmente, o material compósito é utilizado para a fabricação de capots, carters de óleo, colunas de direção, árvores de transmissão, molas laminadas, painéis, etc. Uma das grandes vantagens trazidas para o meio automobilístico pelos materiais compósitos é, além da redução do peso, a facilidade em confeccionar peças com superfícies complexas (ver Figura 2.3). Uma atividade esportiva notória que emprega material compósito é a Fórmula 1, que pode ser considerada como um laboratório para as inovações tecnológicas.

Em muitos casos, o que se emprega dentro dos carros de Fórmula 1 será utilizado futuramente nos carros de passeio. Neste caso, o aumento da relação potência/peso é fundamental para um bom desempenho do carro nas pistas. A configuração mais freqüentemente utilizada nestes carros é do tipo sanduíche que é utilizada para a confecção da carroceria.

Em praticamente todas as atividades esportivas, a redução do peso está diretamente ligada a redução do tempo de execução de uma prova esportiva. Como exemplo disto, pode- 


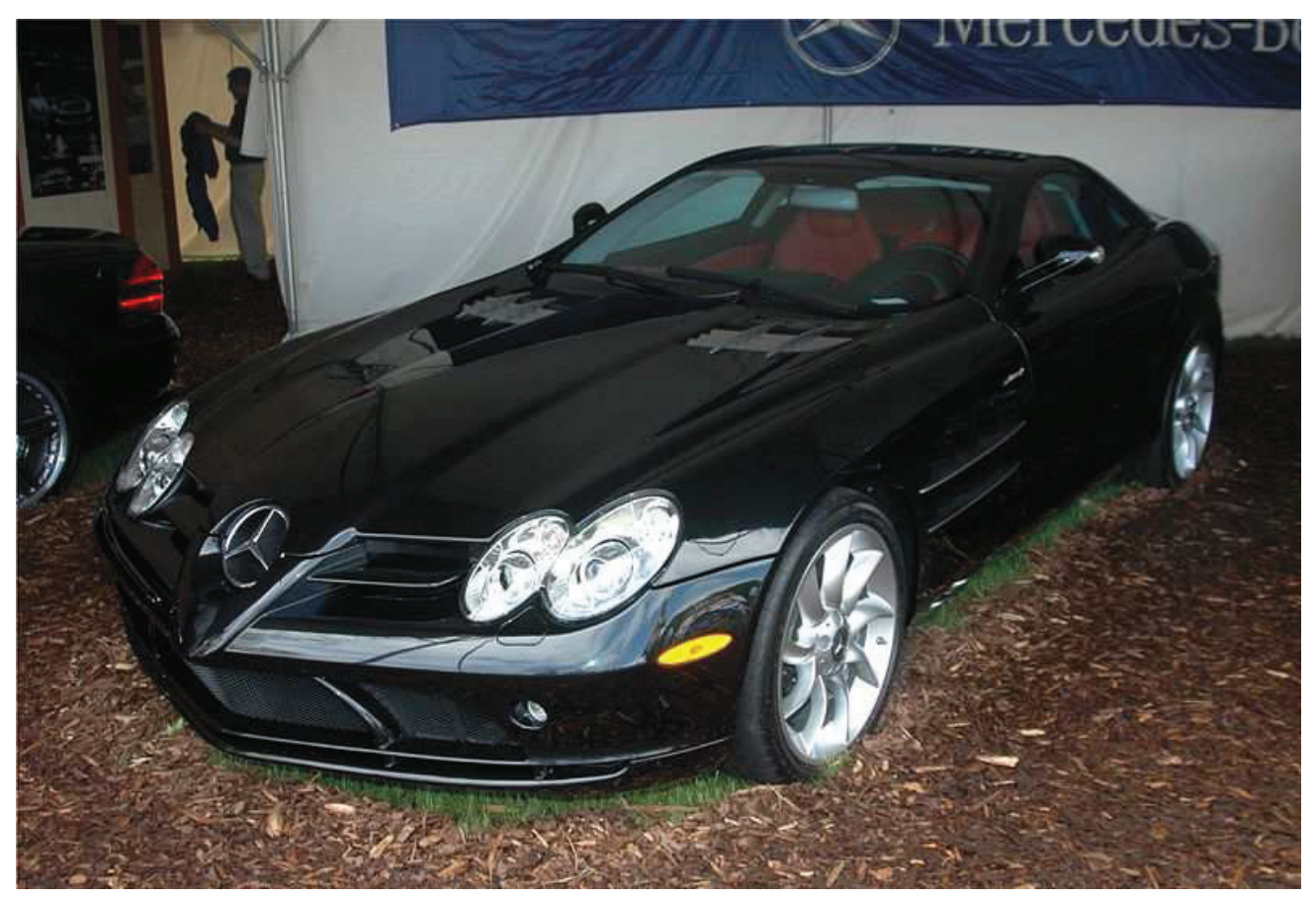

Figura 2.3: Mercedes-Benz SLR McLaren - Chassi construído em fibra de carbono. Crédito: Portal Mercedes-Benz Brasil.

mos citar: barcos a vela, skis, bicicletas, etc. Em alguns casos, o que se procura é a agilidade, e a perfeição, como no tênis, com suas raquetes; no golf, com seus tacos; e no surf, com suas pranchas.

\subsection{Laminados}

Os materiais compósitos são, na maioria dos casos, utilizados na forma de laminados, onde as lâminas são coladas umas sobre as outras com orientações e espessura das fibras podendo ser diferentes uma das outras. Segundo Holmes e Just (1983), as propriedades elásticas de uma lâmina de material compósito reforçado com fibras longas unidirecionais (ver Figura 2.4) podem ser avaliadas a partir de uma visão macroscópica, considerando esta lâmina como um material homogêneo. Assim, qualquer propriedade elástica $(C)$ desta lâmina pode ser expressa como: 


$$
C=C\left(E_{f}, \nu_{f}, V_{f}, E_{m}, \nu_{m}, V_{m}\right)
$$

onde os subscritos $f$ e $m$ referem-se respectivamente à fibra e à matriz. $E$ é o módulo de elasticidade, $\nu$ é a razão de Poisson e $V$ é a proporção em volume de cada componente da lâmina compósita.

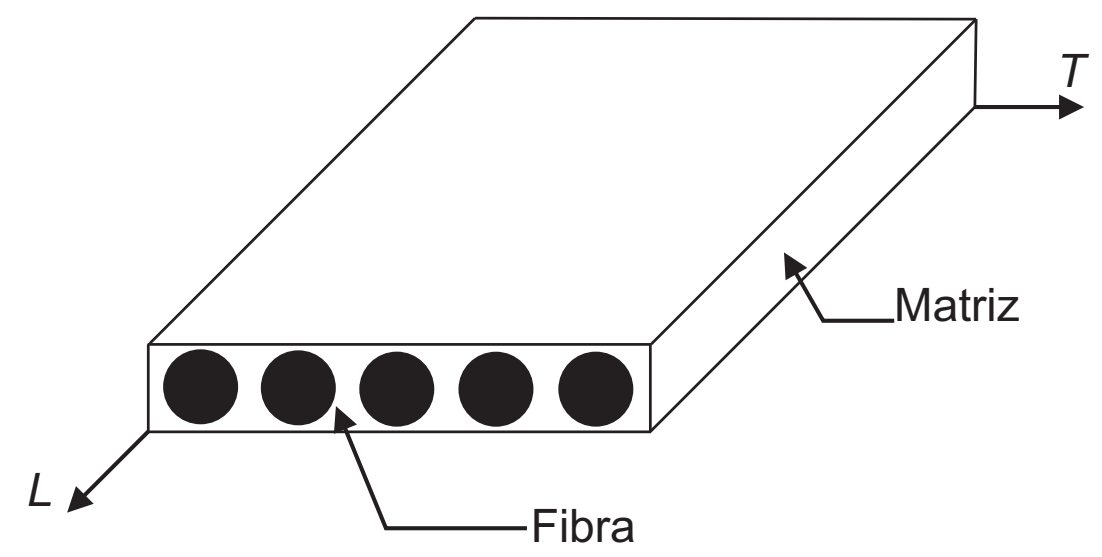

Figura 2.4: Lâmina compósita reforçada com fibras longas unidirecionais.

As propriedades elásticas necessárias para a determinação do comportamento da lâmina, e posteriormente do laminado, são:

- O módulo de elasticidade longitudinal (na direção das fibras) $\left(E_{L}\right)$.

- O módulo de elasticidade transversal (ortogonal às fibras) $\left(E_{T}\right)$.

- O módulo de cisalhamento $\left(G_{L T}\right)$.

- A razão de Poisson principal $\left(\nu_{L T}\right)$.

- A razão de Poisson secundária $\left(\nu_{T L}\right)$.

Os subscritos $L$ e $T$ referem-se respectivamente às direções longitudinal e transversal à direção das fibras. Holmes e Just (1983) apresentam as relações entre as propriedades dos componentes da lâmina, fibra e matriz, e as propriedades da lâmina, dadas por: 


$$
\begin{aligned}
E_{L} & =\left(E_{f}-E_{m}\right) V_{f}+E_{m} \\
\nu_{L T} & =\left(\nu_{f}-\nu_{m}\right) V_{f}+\nu_{m} \\
E_{T} & =\frac{E_{f} E_{m}}{E_{m} V_{f}+E_{f}\left(1-V_{f}\right)} \\
\nu_{T L} & =\nu_{L T} \frac{E_{T}}{E_{L}} \\
G_{L T} & =\frac{G_{f} G_{m}}{G_{m} V_{f}+G_{f}\left(1-V_{f}\right)}
\end{aligned}
$$

onde $G_{f}=\frac{E_{f}}{2\left(1+\nu_{f}\right)}$ e $G_{m}=\frac{E_{m}}{2\left(1-\nu_{m}\right)}$.

Ainda segundo Holmes e Just (1983), estes resultados teóricos são consistentes com resultados experimentais, desconsiderando os efeitos do tempo de ensaio e da temperatura.

\subsection{Equação constitutiva do laminado}

Como apresentado por Agarwal e Broutman (1990), as tensões em cada lâmina podem ser calculadas a partir das deformações através da relação:

$$
\left\{\begin{array}{c}
\sigma_{1} \\
\sigma_{2} \\
\tau_{12}
\end{array}\right\}=\left[\begin{array}{lll}
\bar{Q}_{11} & \bar{Q}_{12} & \bar{Q}_{16} \\
\bar{Q}_{12} & \bar{Q}_{22} & \bar{Q}_{26} \\
\bar{Q}_{16} & \bar{Q}_{26} & \bar{Q}_{66}
\end{array}\right]\left\{\begin{array}{c}
\epsilon_{1} \\
\epsilon_{2} \\
\gamma_{12}
\end{array}\right\}
$$

onde, a matriz $[\bar{Q}]$ é dada por:

$$
[\bar{Q}]=[T]^{-1}[Q][T]
$$

A matriz de transformação $[T]$ é dada por: 


$$
[T]=\left[\begin{array}{ccc}
m^{2} & n^{2} & 2 m n \\
n^{2} & m^{2} & -2 m n \\
-m n & m n & m^{2}-n^{2}
\end{array}\right]
$$

sendo

$$
\begin{aligned}
& m=\cos \theta \\
& n=\sin \theta
\end{aligned}
$$

onde $\theta$ é o ângulo entre as fibras e o sistema de referência (ver Figura 2.5).

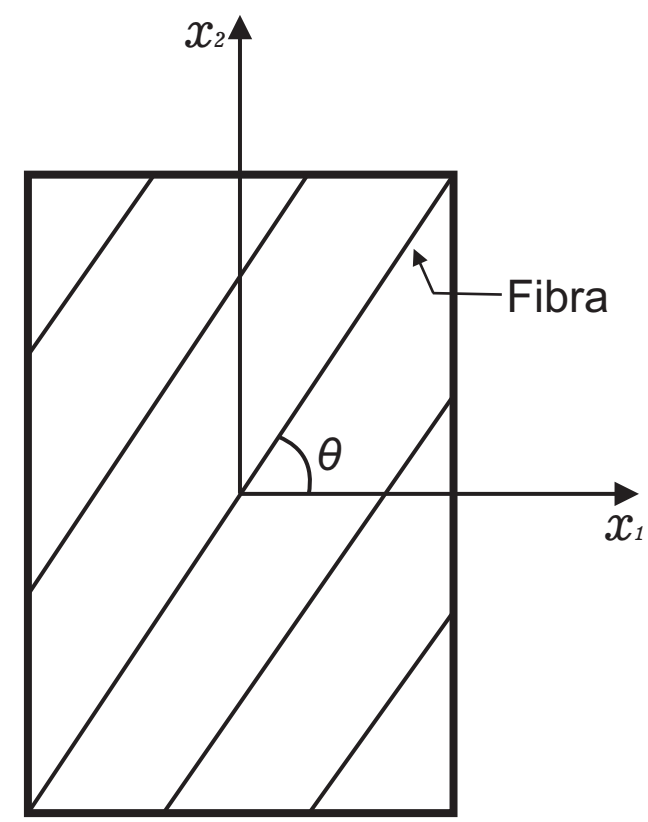

Figura 2.5: Ângulo entre fibras unidirecionais e sistema de referência.

A matriz de rigidez $[Q]$ é dada, em termos das constantes de engenharia por:

$$
[Q]=\left[\begin{array}{ccc}
\frac{E_{L}}{1-\nu_{L T} \nu_{T L}} & \frac{\nu_{L T} E_{T}}{1-\nu_{L T} \nu_{T L}} & 0 \\
\frac{\nu_{L T} E_{T}}{1-\nu_{L T} \nu_{T L}} & \frac{E_{T}}{1-\nu_{L T} \nu_{T L}} & 0 \\
0 & 0 & G_{L T}
\end{array}\right]
$$


As tensões nas direções longitudinal e transversal do reforço com fibras podem ser calculadas por:

$$
\left\{\begin{array}{c}
\sigma_{L} \\
\sigma_{T} \\
\tau_{L T}
\end{array}\right\}=[T]\left\{\begin{array}{c}
\sigma_{1} \\
\sigma_{2} \\
\tau_{12}
\end{array}\right\}
$$




\section{Capítulo 3}

\section{Equações de elasticidade linear}

\subsection{Introdução}

Neste Capítulo é feita uma revisão da teoria da elasticidade aplicada a materiais isotrópicos. É mostrado a relação entre tensões $\times$ deformações e equações de equilíbrio em coordenadas cartesianas e cilíndricas para placas. As formulações presentes neste capítulo serão usadas nos capítulos posteriores na obtenção da formulação de elementos de contorno. Neste trabalho, os índices latinos $i, j$ e $k$ variam de 1 a 3 e os índices gregos $\alpha, \beta$ e $\gamma$ variam de 1 a 2 .

\subsection{Deslocamentos e deformações}

A deformação num corpo sólido contínuo e homogêneo é caracterizada pela extensão de elementos lineares e distorção entre os elementos lineares definidos no domínio do sólido. Este sólido é considerado rígido quando a distância de qualquer par de pontos do seu interior permanece constante durante o carregamento. Assim, pode-se dizer que o movimento de corpo rígido é caracterizado pela translação e rotação. Considere o sólido representado na Figura 3.1. A partir de um sistema de coordenadas cartesianas, as coordenadas do ponto $A$ 
de um sólido inicialmente na posição inicial (não deformado) são denotadas por $\left(x_{1}, x_{2}, x_{3}\right)$. As coordenadas deste mesmo ponto depois da deformação são denotadas por $\left(x_{1}+u_{1}, x_{2}+\right.$ $u_{2}, x_{3}+u_{3}$ ), sendo $u_{1}, u_{2}$ e $u_{3}$ as componentes do vetor deslocamento.

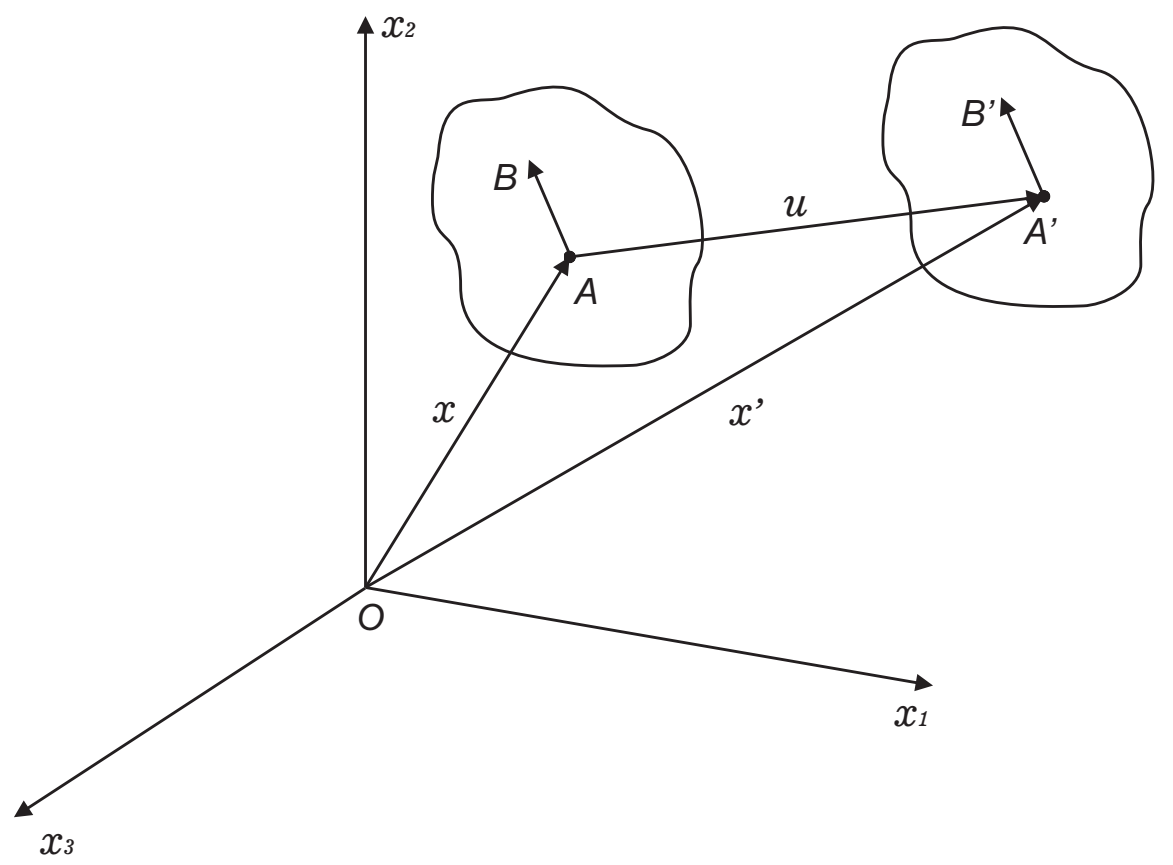

Figura 3.1: Estado de deformação de um sólido.

A posição não deformada correspondente ao ponto $A$ é definida pelo vetor $\mathbf{x}=\overline{O A}$, com componentes $x_{i}{ }^{1}$. A posição $A$ ' corresponde a configuração deformada definida pelo vetor $\mathbf{x}^{\prime}=\overline{O A^{\prime}}$, o ponto $B$ é definido pelo vetor posição $\mathbf{x}+\mathbf{d} \mathbf{x}=\overline{O B}$, cujas componentes são $x_{i}+d x_{i}$ e o ponto $B^{\prime}$ corresponde a configuração deformada cujo vetor posição é $\mathbf{x}^{\prime}+\mathbf{d} \mathbf{x}^{\prime}=$ $\overline{O B^{\prime}}$, cujas componentes são $x_{i}^{\prime}+d x_{i}^{\prime}$. O vetor deslocamento, sofrido por $A$ no sólido é o vetor $\overline{A A^{\prime}}$ o qual é designado por $u$ e cujas componentes são $u_{i}$. O vetor $u$ representa a distância percorrida pelo ponto material entre a posição inicial e a posição final. Sendo $u_{i}$ as componentes do vetor deslocamento $u=\left[u_{1}, u_{2}, u_{3}\right]^{T}$. As coordenadas da posição deformada $A^{\prime}$ podem ser obtidas através da posição não deformada $A$ da seguinte forma:

\footnotetext{
${ }^{1}$ Com $i=1,2$ e 3 .
} 


$$
x_{i}^{\prime}=x_{i}+u_{i}
$$

O comprimento do segmento $\overline{A B}$, é designado por $d s$ :

$$
(d s)^{2}=d x_{i} d x_{i}
$$

A repetição dos índices na equação (3.2) é sinônimo de somatório das parcelas $d x_{1}^{2}, d x_{2}^{2}$ e $d x_{3}^{2}$. O comprimento do vetor $\overline{A^{\prime} B^{\prime}}$ no sólido deformado é designado por $d s^{\prime}$ :

$$
\left(d s^{\prime}\right)^{2}=d x_{i}^{\prime} d x_{i}^{\prime}
$$

A diferença $\left(d s^{\prime}\right)^{2}-(d s)^{2}$ está relacionada com as deformações. Esta diferença pode ser calculada a partir das equações (3.1), (3.2) e (3.3) tendo em conta a formulação de Lagrange para a qual as coordenadas $x_{1}, x_{2}$ e $x_{3}$ são independentes e identificam a posição do ponto na configuração inicial do sólido. Aquela diferença é dada por:

$$
\left(d s^{\prime}\right)^{2}-(d s)^{2}=2 E_{i j} d x_{i} d x_{j}
$$

onde

$$
E_{i j}=\frac{1}{2}\left(\frac{\partial u_{i}}{\partial x_{j}}+\frac{\partial u_{j}}{\partial x_{i}}+\frac{\partial u_{k}}{\partial x_{i}} \frac{\partial u_{k}}{\partial x_{j}}\right)
$$

O tensor $E_{i j}$ é designado por tensor das deformações de Lagrange. A equação (3.5) define as deformações com base na configuração inicial do sólido e medida no sistema de eixos inicial. A deformação com base na configuração deformada do sólido é dada por:

$$
\left(d s^{\prime}\right)^{2}-(d s)^{2}=2 E_{i j}^{\prime} d x_{i}^{\prime} d x_{j}^{\prime}
$$

onde 


$$
E_{i j}^{\prime}=\frac{1}{2}\left(\frac{\partial u_{i}}{\partial x_{j}^{\prime}}+\frac{\partial u_{j}}{\partial x_{i}^{\prime}}-\frac{\partial u_{k}}{\partial x_{i}^{\prime}} \frac{\partial u_{k}}{\partial x_{j}^{\prime}}\right)
$$

O tensor $E_{i j}^{\prime}$ é designado por tensor das deformações de Almansi. A equação (3.7) define as deformações com base na configuração final do sólido. Para grandes deformações pode-se usar o tensor das deformações de Lagrange ou o tensor das deformações de Almansi. Para pequenas deformações são desprezados os termos de segunda ordem das equações (3.5) e (3.7), tornando-se dessa forma desnecessário distinguir entre as deformações de Lagrange e Almansi. Dessa forma temos as deformações consideradas como lineares e escritas do seguinte modo:

$$
\varepsilon_{i j}=\frac{1}{2}\left(\frac{\partial u_{i}}{\partial x_{j}}+\frac{\partial u_{j}}{\partial x_{i}}\right)
$$

O tensor das deformações lineares, equação (3.8), apresenta nove componentes e pode ser expresso através da matriz $(3 \times 3)$ abaixo:

$$
\left[\begin{array}{ccc}
\varepsilon_{11} & \varepsilon_{12} & \varepsilon_{13} \\
\varepsilon_{21} & \varepsilon_{22} & \varepsilon_{23} \\
\varepsilon_{31} & \varepsilon_{32} & \varepsilon_{33}
\end{array}\right]=\left[\begin{array}{ccc}
\frac{\partial u_{1}}{\partial x_{1}} & \frac{1}{2}\left(\frac{\partial u_{1}}{\partial x_{2}}+\frac{\partial u_{2}}{\partial x_{1}}\right) & \frac{1}{2}\left(\frac{\partial u_{1}}{\partial x_{3}}+\frac{\partial u_{3}}{\partial x_{1}}\right) \\
\frac{1}{2}\left(\frac{\partial u_{1}}{\partial x_{2}}+\frac{\partial u_{2}}{\partial x_{1}}\right) & \frac{\partial u_{2}}{\partial x_{2}} & \frac{1}{2}\left(\frac{\partial u_{2}}{\partial x_{3}}+\frac{\partial u_{3}}{\partial x_{2}}\right) \\
\frac{1}{2}\left(\frac{\partial u_{1}}{\partial x_{3}}+\frac{\partial u_{3}}{\partial x_{1}}\right) & \frac{1}{2}\left(\frac{\partial u_{2}}{\partial x_{3}}+\frac{\partial u_{3}}{\partial x_{2}}\right) & \frac{\partial u_{3}}{\partial x_{3}}
\end{array}\right]
$$

As componentes de deformação $\varepsilon_{11}, \varepsilon_{22}$ e $\varepsilon_{33}$ correspondem as extensões nas direções dos eixos $x_{1}, x_{2}$ e $x_{3}$. As componentes $\varepsilon_{12}, \varepsilon_{13}, \varepsilon_{23}, \varepsilon_{21}, \varepsilon_{31}$ e $\varepsilon_{32}$ correspondem as distorções ou deformações por cisalhamento. O tensor de deformações, $\varepsilon_{i j}$ num ponto qualquer do sólido, com base na equação (3.9), é obtido a partir do vetor de deslocamentos, $u_{i}$ num ponto. O problema inverso também pode ser obtido. O número de deformações independentes na equação (3.9) é seis e o número de deslocamentos é três, dessa forma, o número de equações a integrar é superior ao número de deslocamentos a obter, sendo necessário estabelecer condições de integrabilidade das deformações, e estas condições são chamadas de condições de compatibilidade e são dadas por: 


$$
\varepsilon_{i j, k l}+\varepsilon_{k l, i j}-\varepsilon_{i k, j l}-\varepsilon_{j l, i k}=0
$$

A equação (3.10), também chamada de equação tensorial, corresponde a 81 equações das quais apenas seis são independentes, e as demais representam identidades ou repetições que surgem em consequência da simetria do tensor de deformações.

\subsection{Tensões e equações de equilíbrio}

A existência de deformação não é independente da ocorrência de tensões que podem ser definidas pelo tensor das tensões. No caso de se considerar pequenas deformações, é suficiente considerar-se só um tensor das tensões, uma vez que nessas condições há coincidência entre as deformações de Lagrange e Almansi. De igual modo não se destinguem as tensões na configuração inicial e as tensões na configuração deformada do sólido. Considere um elemento infinitesimal de volume $d V$ com as superfícies paralelas aos eixos coordenados (ver Figura 3.2). As tensões atuando em três faces ortogonais são nove e constituem o chamado tensor das tensões que é representado sobre a forma matricial:

$$
\sigma_{i j}=\left[\begin{array}{ccc}
\sigma_{11} & \sigma_{12} & \sigma_{13} \\
\sigma_{21} & \sigma_{22} & \sigma_{23} \\
\sigma_{31} & \sigma_{32} & \sigma_{33}
\end{array}\right]
$$

onde o primeiro índice diz respeito à direção da normal ao plano que está sendo considerado e o segundo índice diz respeito à direção da tensão. As tensões $\sigma_{11}, \sigma_{22}$ e $\sigma_{33}$ são tensões normais e as tensões $\sigma_{12}, \sigma_{13}, \sigma_{21}, \sigma_{23}, \sigma_{31}$ e $\sigma_{32}$ são tensões de cisalhamento ou tangenciais. Nas outras arestas do elemento é possível considerar tensões que são:

$$
\sigma_{11}+\frac{\partial \sigma_{11}}{\partial x_{1}} d x_{1}
$$




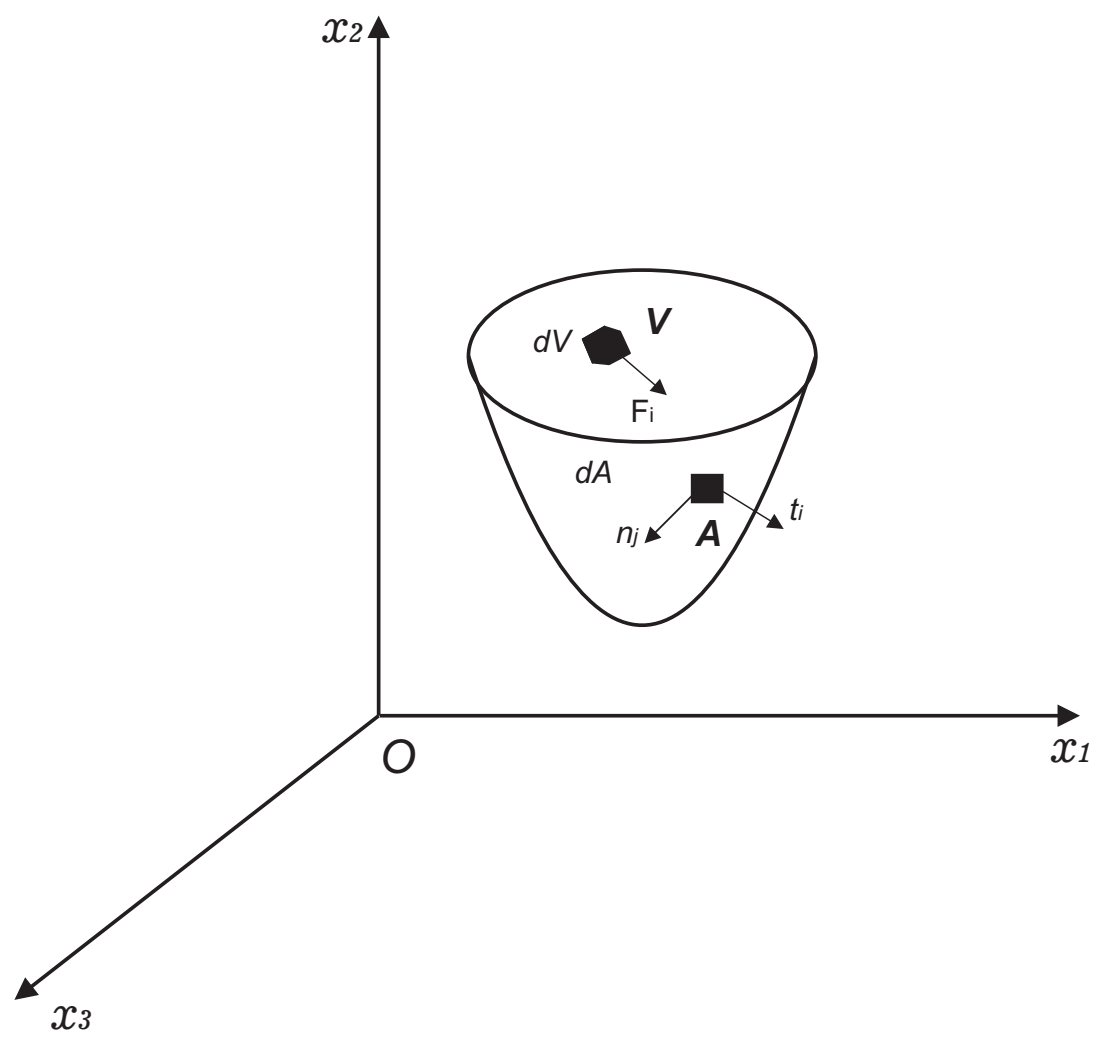

Figura 3.2: Forças de superfície e volume.

$$
\begin{gathered}
\sigma_{22}+\frac{\partial \sigma_{22}}{\partial x_{2}} d x_{2} \\
\sigma_{12}+\frac{\partial \sigma_{12}}{\partial x_{1}} d x_{1}
\end{gathered}
$$

Considerando as forças aplicadas às seis faces do elemento infinitesimal obtemos as seguintes equações:

$$
\begin{aligned}
& \frac{\partial \sigma_{11}}{\partial x_{1}}+\frac{\partial \sigma_{12}}{\partial x_{2}}+\frac{\partial \sigma_{13}}{\partial x_{3}}+F_{1}=0 \\
& \frac{\partial \sigma_{21}}{\partial x_{1}}+\frac{\partial \sigma_{22}}{\partial x_{2}}+\frac{\partial \sigma_{23}}{\partial x_{3}}+F_{2}=0
\end{aligned}
$$




$$
\frac{\partial \sigma_{31}}{\partial x_{1}}+\frac{\partial \sigma_{32}}{\partial x_{2}}+\frac{\partial \sigma_{33}}{\partial x_{3}}+F_{3}=0
$$

As equações (3.15) à (3.17) satisfazem as condições de equilíbrio das forças atuantes num elemento infinitesimal em termos das tensões nas vizinhanças dos pontos. A equação de equilíbrio tambem pode ser escrita da seguinte forma:

$$
\sigma_{i j, j}+F_{i}=0
$$

As forças de superfície agindo em um elemento infinitesimal de área $d A$ (Figura 3.2), na superfície do sólido em uma direção $n_{j}$, normal a superfície são dados por:

$$
t_{i}=\sigma_{i j} n_{j}
$$

sendo $n_{j}$ os cossenos diretores dos ângulos formados pelo vetor normal e os eixos coordenados $x_{i}$ (Dinis, 2004).

\subsection{Equação constitutiva}

Em uma região elástica linear de um sólido contínuo e homogêneo, a equação que relaciona o tensor das tensões $\left(\sigma_{i j}\right)$ com o tensor das deformações $\left(\varepsilon_{i j}\right)$ é a chamada lei de Hooke. Em qualquer ponto do sólido, as componentes de tensão relacionam-se com as componentes de deformação da seguinte forma:

$$
\sigma_{i j}=C_{i j k l} \varepsilon_{k l}
$$

onde o tensor de quarta ordem $C_{i j k l}$ é formado por componentes que contém as constantes elásticas do material. O tensor $C_{i j k l}$ é composto por 81 constantes elásticas. Porém, se for levado em consideração a simetria dos tensores de segunda ordem envolvidos, a existência 
de funções de densidade de energia e as propriedades de isotropia, as constantes podem ser resumidas a apenas duas $(E, \nu)$. Desta forma, a equação (3.20) pode ser escrita da seguinte forma:

$$
\sigma_{i j}=\frac{E \nu}{(1+\nu)(1-2 \nu)} \delta_{i j} \varepsilon_{k k}+\frac{E}{(1+\nu)} \varepsilon_{i j}
$$

onde

$\delta_{i j}$ : delta de Kronecker,

$E$ : módulo de elasticidade,

$\nu$ : coeficiente de Poisson,

$G$ : módulo de cisalhamento, $G=\frac{E}{2(1+\nu)}$.

A equação (3.21) pode ser escrita em termos de deformações da seguinte forma:

$$
\varepsilon_{i j}=\frac{1+\nu}{E} \sigma_{i j}-\frac{\nu}{E} \delta_{i j} \sigma_{k k}
$$

\subsection{Relações de tensão $\times$ deformação e deslocamento em placas}

Considere um sistema de coordenadas cartesianas mostrado na Figura 3.3 onde relacionase coordenadas cartesianas $x_{1}, x_{2}$ e $x_{3}$ com coordenadas cilíndricas $r, \theta$ e $x_{3}$ de forma a escrever as relações básicas para teoria de flexão de placas.

Com base na equação (3.8), para uma placa sujeita a carregamentos normais em sua superfície, os deslocamentos resultantes em sua superfície média são dados por $u, \nu$ e $w$. Assim, através de um sistema de coordenadas cartesianas, as componentes de deformação em função dos deslocamentos podem ser escritas da seguinte forma: 


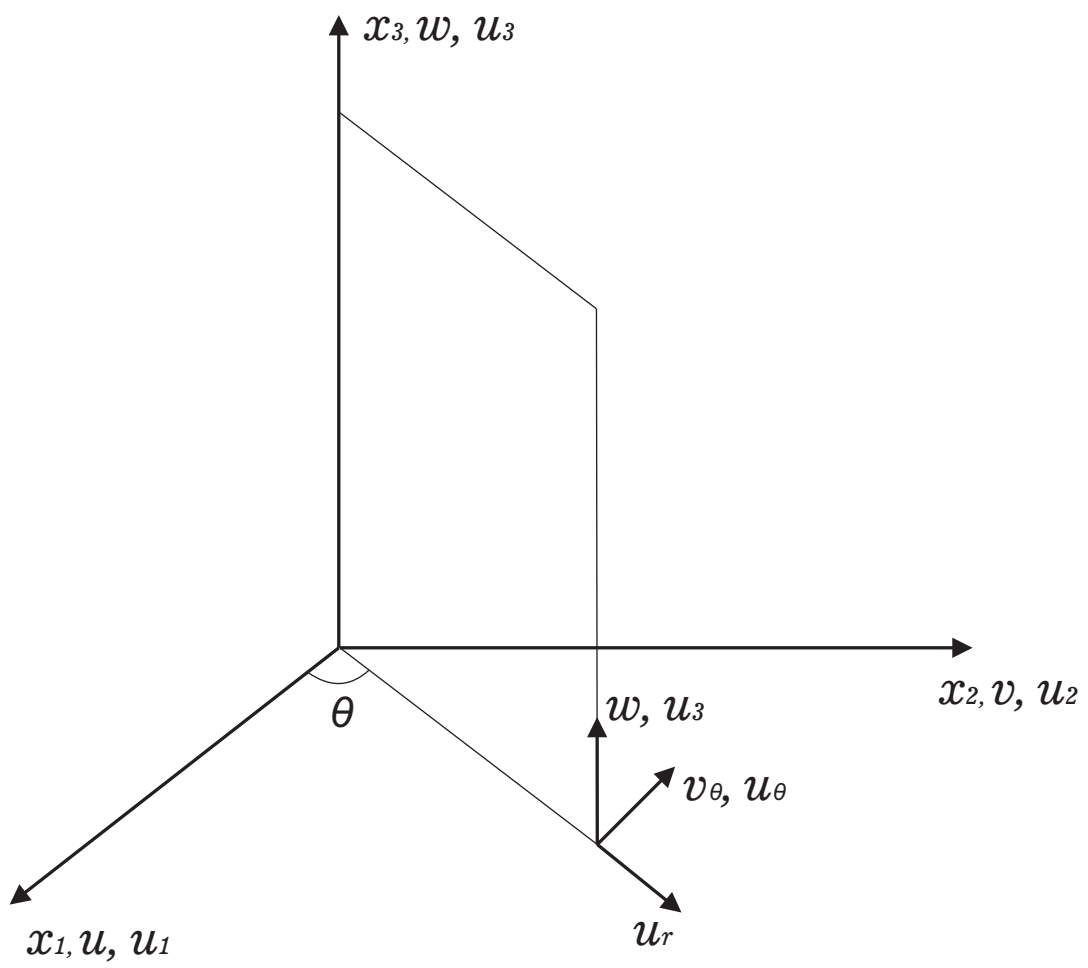

Figura 3.3: Sistema de coordenadas cilíndricas.

$$
\left\{\begin{array}{c}
\varepsilon_{11} \\
\varepsilon_{22} \\
\varepsilon_{33}
\end{array}\right\}=\left\{\begin{array}{c}
\frac{\partial u}{\partial x_{1}} \\
\frac{\partial \nu}{\partial x_{2}} \\
\frac{\partial w}{\partial x_{3}}
\end{array}\right\}
$$

para as deformações lineares e

$$
\left\{\begin{array}{c}
\gamma_{12} \\
\gamma_{13} \\
\gamma_{23}
\end{array}\right\}=\left\{\begin{array}{c}
\frac{\partial \nu}{\partial x_{1}}+\frac{\partial u}{\partial x_{2}} \\
\frac{\partial u}{\partial x_{3}}+\frac{\partial w}{\partial x_{1}} \\
\frac{\partial w}{\partial x_{2}}+\frac{\partial \nu}{\partial x_{3}}
\end{array}\right\}
$$

para as distorções ou deformações angulares.

Os deslocamentos em coordenadas cilíndricas devido um carregamento aplicado são dados por $u_{r}, v_{\theta}$ e $w$ : 


$$
\begin{gathered}
\left\{\begin{array}{c}
\varepsilon_{r r} \\
\varepsilon_{\theta \theta} \\
\varepsilon_{33}
\end{array}\right\}=\left\{\begin{array}{c}
\frac{\partial u_{r}}{\partial r} \\
\frac{1}{r} \frac{\partial \nu_{\theta}}{\partial \theta}+\frac{u_{r}}{r} \\
\frac{\partial w}{\partial x_{3}}
\end{array}\right\} \\
\left\{\begin{array}{c}
\gamma_{r \theta} \\
\gamma_{r 3} \\
\gamma_{\theta 3}
\end{array}\right\}=\left\{\begin{array}{c}
\frac{1}{r} \frac{\partial u_{r}}{\partial \theta}+\frac{\partial \nu_{\theta}}{\partial r}-\frac{\nu_{\theta}}{r} \\
\frac{\partial u_{r}}{\partial x_{3}}+\frac{\partial w}{\partial r} \\
\frac{\partial \nu_{\theta}}{\partial x_{3}}+\frac{1}{r} \frac{\partial w}{\partial \theta}
\end{array}\right\}
\end{gathered}
$$

\subsection{Equações de equilíbrio em coordenadas cilíndricas}

O equilíbrio estático de um elemento infinitesimal onde atuam em cada ponto um conjunto de tensões internas é mostrado na Figura 3.4.

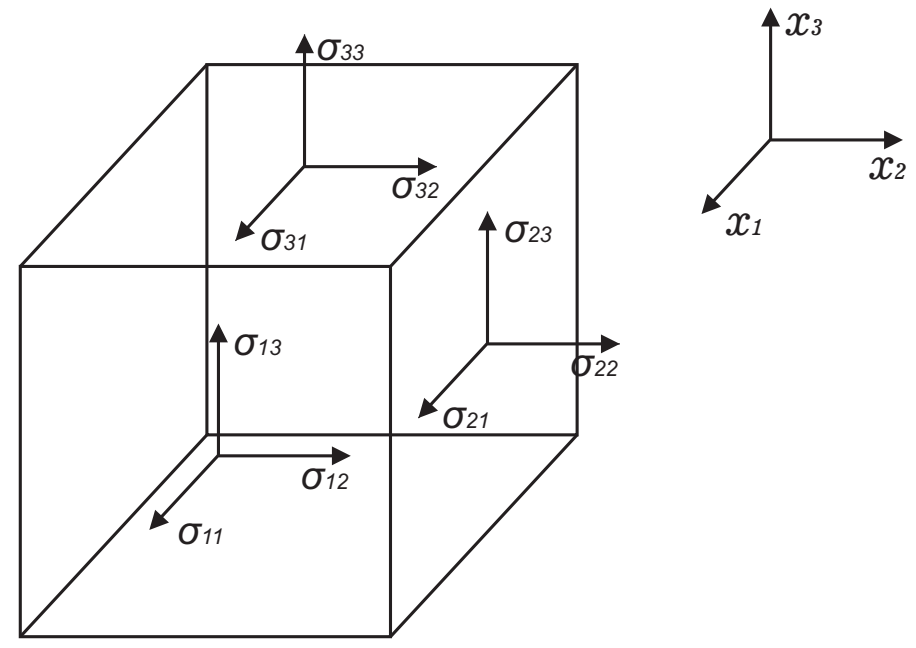

Figura 3.4: Elemento infinitesimal.

Como mostrado no item 3.3, onde o elemento sólido está sujeito a forças volumétricas $F_{i}$ e forças de superfície $t_{i}$, foram obtidas as equações de equilíbrio expressas pelas equações (3.15) à (3.17) em coordenadas cartesianas. Em coordenadas cilíndricas as equações de equilíbrio são dadas por:

$$
\frac{\partial \sigma_{r r}}{\partial r}+\frac{1}{r} \frac{\partial \sigma_{\theta r}}{\partial \theta}+\frac{\partial \sigma_{3 r}}{\partial x_{3}}+\frac{1}{r}\left(\sigma_{r r}-\sigma_{\theta \theta}\right)+F_{r}=0
$$




$$
\begin{aligned}
& \frac{\partial \sigma_{r \theta}}{\partial r}+\frac{1}{r} \frac{\partial \sigma_{\theta \theta}}{\partial \theta}+\frac{\partial \sigma_{3 \theta}}{\partial x_{3}}+\frac{2}{r} \sigma_{r \theta}+F_{\theta}=0 \\
& \frac{\partial \sigma_{r 3}}{\partial r}+\frac{1}{r} \frac{\partial \sigma_{\theta 3}}{\partial \theta}+\frac{\partial \sigma_{33}}{\partial x_{3}}+\frac{1}{r} \sigma_{r 3}+F_{3}=0
\end{aligned}
$$




\section{Capítulo 4}

\section{Placas espessas isotrópicas}

\subsection{Introdução}

Neste Capítulo é obtida a equação de equilíbrio para placas espessas isotrópicas. A teoria de placas é simplificada para um modelo bi-dimensional a partir do modelo original tri-dimensional. A idéia básica da teoria de placas é assumir a distribuição de tensões através da espessura, como se faz na teoria de tensões de Reissner, ou assumir a distribuição de deformações através da espessura como na teoria baseada nos deslocamentos proposta por Mindlin. Tanto a teoria de Reissner quanto a de Mindlin são teorias de placas espessas, por levarem em consideração a deformação de cisalhamento ao longo da direção transversal. Estas teorias descrevem as deformações ao longo da espessura através de uma função de primeira ordem, ou seja, função linear.

\subsection{Teoria de Mindlin}

A teoria de placas espessas de Mindlin (1951) surge em consequência da existência de placas que não podem ser consideradas finas, pois os efeitos das tensões de cisalhamento transversais podem ser significativos. Para este tipo de placas, as hipóteses de Kirchhoff 
consideradas válidas para as placas finas deixam de ser admissíveis (Kirchhoff, 1950). A teoria de placas pode ser modificada considerando-se que a espessura tem dimensões mais elevadas. Considere uma placa arbitrária de espessura $h$, como mostrado na Figura 4.1, com domínio $\Omega$ e contorno $\Gamma$ no espaço $x_{i}{ }^{1}$. O sistema $O x_{1} x_{2} x_{3}$, o qual é definido como o plano médio da placa antes da deformação coincide com $O x_{1} x_{2}$, onde $x_{3}=0$. Os deslocamentos generalizados são denotados como $u_{i}$, onde $u_{\alpha}(\alpha=1,2)$ denota rotações $\left(\theta_{1}\right.$ e $\left.\theta_{2}\right)$ e $u_{3}$ denota a deflexão transversal $w$ (ver Figura 4.2).
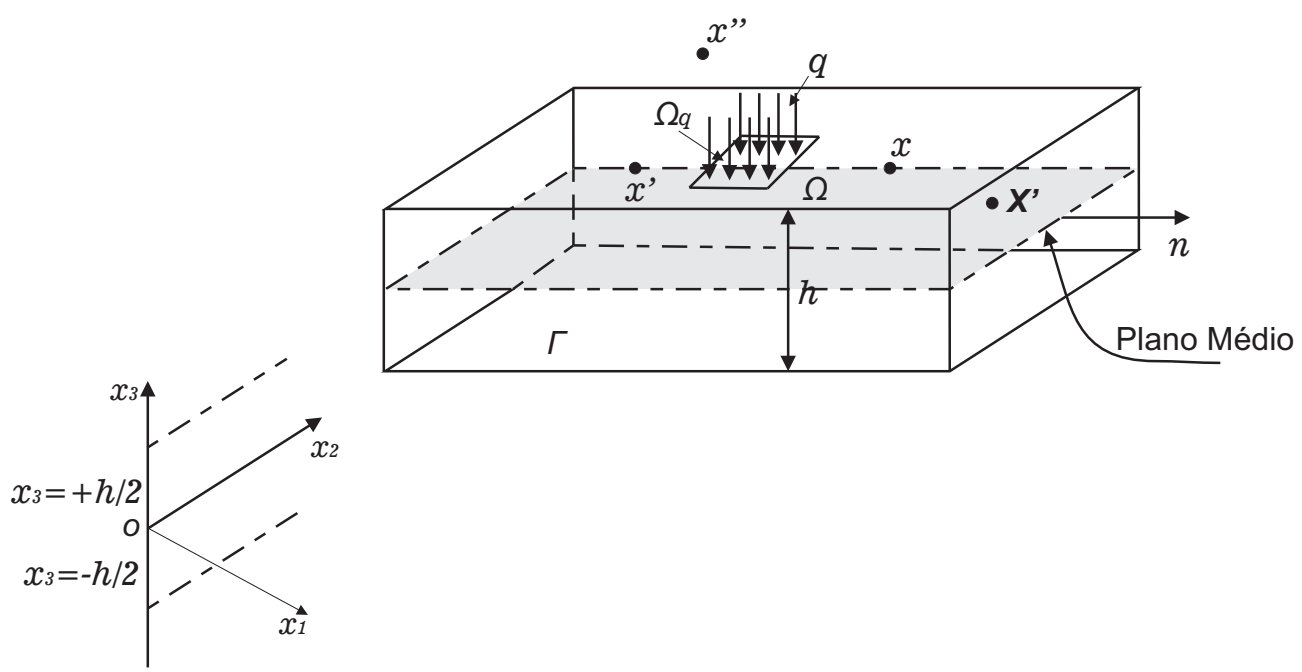

Figura 4.1: Geometria da placa.

As hipóteses de Mindlin para placas espessas isotrópicas, utilizadas para representar deformações e tensões quando submetidas a carregamentos normais ao plano médio, são:

1. A superfície média é plana e indeformável, ou seja, as deformações no plano são nulas: $\varepsilon_{11}=\varepsilon_{22}=\varepsilon_{12}=0$.

2. Os pontos pertencentes à normal ao plano médio da placa depois da deformação de flexão permanecem numa direção linear mas não normal ao plano médio.

3. As tensões na direção normal ao plano médio, $\sigma_{33}$, são desprezivéis em comparação as tensões $\sigma_{11}$ e $\sigma_{22}$. Assim, considera-se que as deformações à normal são: $\varepsilon_{33}=0$.

\footnotetext{
${ }^{1} \mathrm{Com} i=1,2$ e 3 .
} 


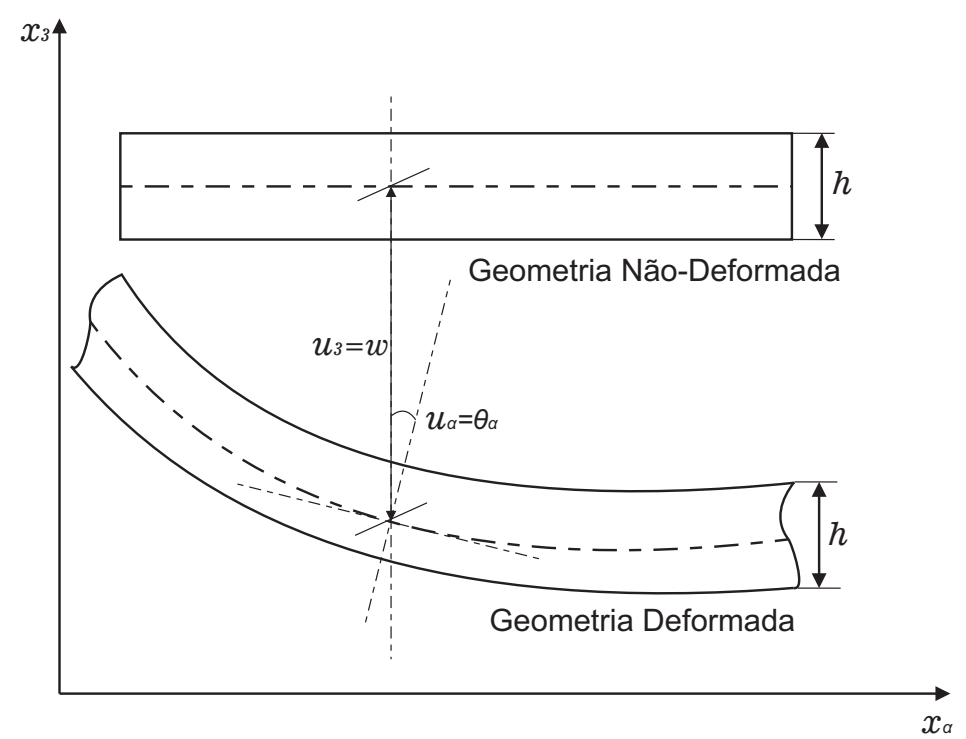

Figura 4.2: Deslocamentos generalizados.

Neste caso, o tensor de tensões para teoria clássica de placas é dado por:

$$
\sigma_{i j}=\left[\begin{array}{ccc}
\sigma_{11} & \sigma_{12} & \sigma_{13} \\
\sigma_{21} & \sigma_{22} & \sigma_{23} \\
\sigma_{31} & \sigma_{32} & \sigma_{33}
\end{array}\right]
$$

Levando-se em consideração a hipótese 2 , os deslocamentos $u_{1}$ e $u_{2}$ de um ponto $P$ da placa situado a uma distância $x_{3}$ do plano médio podem ser calculados a partir dos valores das rotações $\theta_{1}$ e $\theta_{2}$ da normal que, após a deformação, se admitiu ser linear mas não normal ao plano médio, conforme mostrado na Figura 4.2. O vetor de deslocamentos $\left(u_{1}, u_{2}, u_{3}\right)^{T}$.

$$
\left\{\begin{array}{l}
u_{1} \\
u_{2} \\
u_{3}
\end{array}\right\}=-\left\{\begin{array}{c}
-x_{3} \theta_{1} \\
-x_{3} \theta_{2} \\
\omega\left(x_{1}, x_{2}\right)
\end{array}\right\}
$$

Dessa forma, as deformações no plano $O x_{1} x_{2}$ para placa sujeita a flexão a uma distância $x_{3}$ do plano médio da placa podem ser obtidas através das equações (3.8) e (4.2) da seguinte forma: 


$$
\left\{\begin{array}{c}
\varepsilon_{11} \\
\varepsilon_{22} \\
\varepsilon_{12}
\end{array}\right\}=\left\{\begin{array}{c}
-x_{3} \frac{\partial \theta_{1}}{\partial x_{1}} \\
-x_{3} \frac{\partial \theta_{2}}{\partial x_{2}} \\
-x_{3} \frac{\partial \theta_{1}}{\partial x_{2}}-x_{3} \frac{\partial \theta_{2}}{\partial x_{1}}
\end{array}\right\}
$$

As deformações de cisalhamento $\gamma_{13}$ e $\gamma_{23}$ são dadas por:

$$
\left\{\begin{array}{c}
\gamma_{13} \\
\gamma_{23}
\end{array}\right\}=\left\{\begin{array}{l}
-\theta_{1}+\frac{\partial \omega}{\partial x_{1}} \\
-\theta_{2}+\frac{\partial \omega}{\partial x_{2}}
\end{array}\right\}
$$

Na superfície média a coordenada, $x_{3}=0$, temos:

$$
\varepsilon_{11}=\varepsilon_{22}=\varepsilon_{12}=0
$$

As deformações de cisalhamento transversais são consideradas constantes ao longo da espessura e são diferentes de zero, contrariamente a teoria proposta por Kirchhoff. As deformações $\varepsilon_{11}$, $\varepsilon_{22}$ e $\varepsilon_{21}$ variam linearmente ao longo da espessura da placa, o que está de acordo com as hipóteses de Mindlin.

As tensões $\sigma_{11}, \sigma_{22}$ e $\sigma_{12}$ variam linearmente ao longo do eixo $O x_{3}$ como mostra a Figura 4.3 , sendo que para $x_{3}=0$ as tensões tornam-se nulas de acordo com a teoria de Mindlin. As tensões de cisalhamento são constantes ao longo da espessura. Esta aproximação das tensões de cisalhamento contrariam o fato da tensão de cisalhamento se anularem na superfície da placa, ou seja, quando $x_{3}=-h / 2$ e $x_{3}=h / 2$, o que sugere a possibilidade de se considerar teorias que sejam de ordem superior.

\subsection{Esforços generalizados e curvaturas}

Os esforços unitários, os momentos fletores, $M_{11}$ e $M_{22}$, o momento torçor $M_{12}$ e forças cisalhantes $Q_{1}$ e $Q_{2}$ são calculados de modo análogo ao considerado no caso de placas finas. 

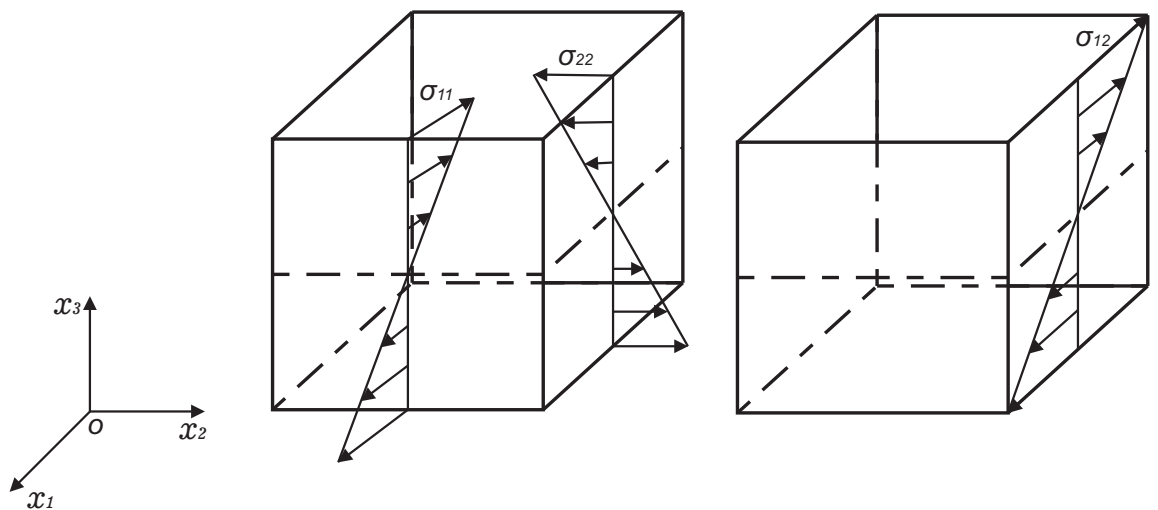

Figura 4.3: Distribuição de tensões ao longo da espessura.

O momento fletor $M_{11}$, é o momento resultante por unidade de comprimento na direção $O x_{1}$ das tensões $\sigma_{11}$ ao longo da espessura da placa, ou seja:

$$
M_{11}=\int_{-h / 2}^{h / 2} \sigma_{11} x_{3} d x_{3}
$$

De modo semelhante se definem os momentos fletores, $M_{22}$ e $M_{12}$ que resultam das tensões $\sigma_{22}$ e $\sigma_{12}$, ou seja:

$$
\begin{aligned}
& M_{22}=\int_{-h / 2}^{h / 2} \sigma_{22} x_{3} d x_{3} \\
& M_{12}=\int_{-h / 2}^{h / 2} \sigma_{12} x_{3} d x_{3}
\end{aligned}
$$

As forças cortantes definem-se a partir das tensões $\sigma_{13}$ e $\sigma_{23}$ da seguinte forma:

$$
\begin{aligned}
& Q_{1}=\int_{-h / 2}^{h / 2} \sigma_{13} d x_{3} \\
& Q_{2}=\int_{-h / 2}^{h / 2} \sigma_{23} d x_{3}
\end{aligned}
$$


Integrando as equações (4.5) à (4.7) para os momentos, obtém-se:

$$
\begin{gathered}
M_{11}=-D\left(\frac{\partial \theta_{1}}{\partial x_{1}}+\nu \frac{\partial \theta_{2}}{\partial x_{2}}\right) \\
M_{22}=-D\left(\frac{\partial \theta_{2}}{\partial x_{2}}+\nu \frac{\partial \theta_{1}}{\partial x_{1}}\right) \\
M_{12}=\frac{1-\nu}{2} D\left(\frac{\partial \theta_{1}}{\partial x_{2}}+\nu \frac{\partial \theta_{2}}{\partial x_{1}}\right)
\end{gathered}
$$

sendo $D=E h^{3} / 12\left(1-\nu^{2}\right)$, o módulo de rigidez à flexão da placa. As curvaturas da superfície fletida podem ser expressas por $\chi_{11}, \chi_{22}$ e $\chi_{12}$ :

$$
\begin{gathered}
\chi_{11}=\partial \theta_{1} / \partial x_{1} \\
\chi_{22}=\partial \theta_{2} / \partial x_{2}
\end{gathered}
$$

$$
\chi_{12}=\partial \theta_{1} / \partial x_{2}+\partial \theta_{2} / \partial x_{1}
$$

As equações (4.10), (4.11) e (4.12) podem ser expressas da seguinte forma:

$$
\left\{\begin{array}{l}
M_{11} \\
M_{22} \\
M_{12}
\end{array}\right\}=-D\left[\begin{array}{ccc}
1 & \nu & 0 \\
\nu & 1 & 0 \\
0 & 0 & (1-\nu / 2)
\end{array}\right]\left\{\begin{array}{l}
\chi_{11} \\
\chi_{22} \\
\chi_{12}
\end{array}\right\}
$$

A contribuição das rotações para as deformações devido as forças cortantes podem ser dadas por: 


$$
\left\{\begin{array}{l}
\phi_{1} \\
\phi_{2}
\end{array}\right\}=\left\{\begin{array}{l}
-\theta_{1}+\frac{\partial \omega}{\partial x_{1}} \\
-\theta_{2}+\frac{\partial \omega}{\partial x_{2}}
\end{array}\right\}
$$

Os esforços cortantes tomam a seguinte forma em função das rotações:

$$
\left\{\begin{array}{l}
Q_{1} \\
Q_{2}
\end{array}\right\}=G^{\prime}\left[\begin{array}{ll}
1 & 0 \\
0 & 1
\end{array}\right]\left\{\begin{array}{l}
\phi_{1} \\
\phi_{2}
\end{array}\right\}
$$

onde $G^{\prime}=E h / 2(1+\nu)$.

No cálculo dos esforços cortantes é por vezes usual incluir o fator de correção a fim de melhor representar os esforços de cisalhamento. Este fator é multiplicado pelos esforços acabados de calcular e tem o valor de 5/6. Pode-se notar que a partir das deformações de cisalhamento, equação (4.17), obtemos os esforços cortantes, equação (4.18), conduzindo assim as tensões de cisalhamento, que são constantes ao longo da espessura. Os momentos fletores $M_{\alpha \beta}$ e os esforços cortantes $Q_{\alpha}$ podem ser escritos em notação indicial da seguinte maneira:

$$
\begin{gathered}
M_{\alpha \beta}=\int_{-h / 2}^{h / 2} x_{3} \sigma_{\alpha \beta} d x_{3} \\
Q_{\alpha}=\int_{-h / 2}^{h / 2} x_{3} \sigma_{\alpha 3} d x_{3}
\end{gathered}
$$

onde $\sigma_{\alpha \beta}$ são componentes da tensão normal através da espessura da placa e $\sigma_{\alpha 3}$ são as componentes da tensão de cisalhamento transversal. As forças de superfície generalizadas em um ponto do contorno, $x \in \Gamma$, podem ser definidas como:

$$
\begin{gathered}
p_{\alpha}=M_{\alpha \beta} n_{\beta} \\
p_{3}=Q_{\alpha} n_{\alpha}
\end{gathered}
$$


onde $n_{\beta}$ e $n_{\alpha}$ são as componentes do vetor normal ao contorno $\Gamma$ da placa. A Figura 4.4 mostra a convenção de sinal para as forças de superfície generalizadas.

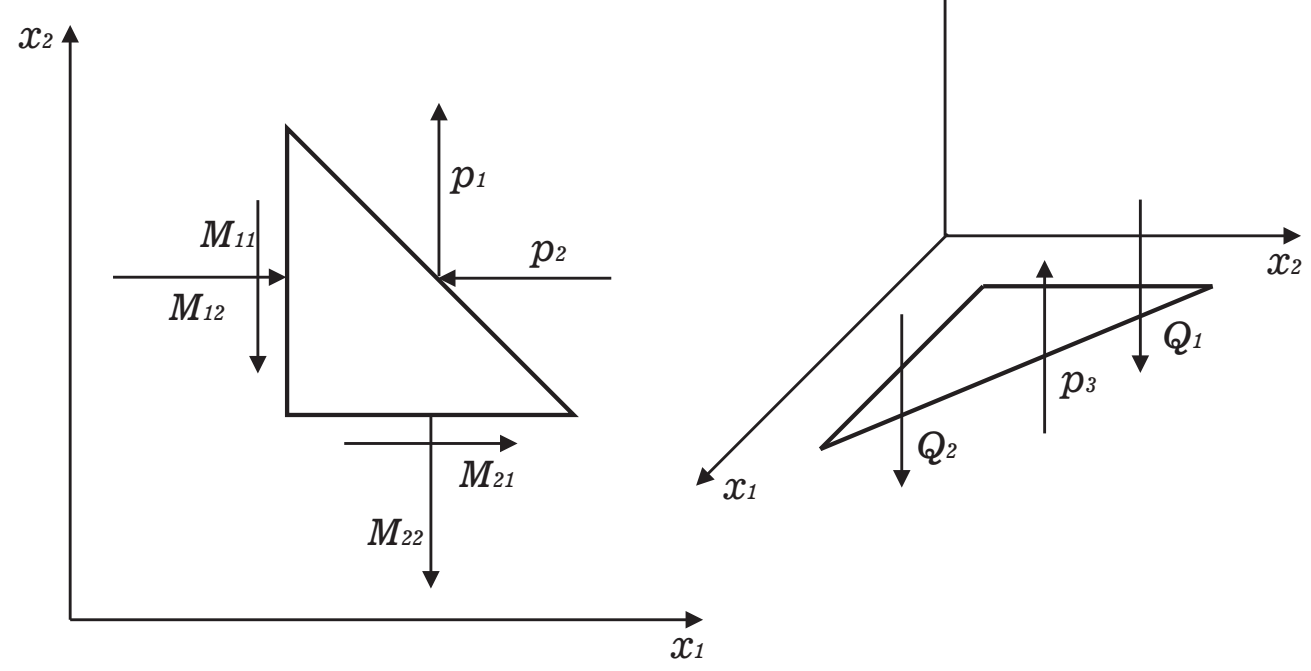

Figura 4.4: Convenção de sinal para forças de superfície generalizadas em pontos do contorno.

\subsection{Trabalho virtual}

O trabalho interno virtual das tensões $\sigma_{i j}$ para uma deformação virtual $\varepsilon_{i j}^{-}$é definido da seguinte forma (Dinis, 2004):

$$
\delta T=\int_{V} \sigma_{i j} \bar{\varepsilon}_{i j} d V
$$

sendo a integração estendida ao volume da placa $d V$. Tendo em conta as equações (4.3) e (4.4) que definem as tensões e as deformações em termos dos deslocamentos e rotações, a expressão do trabalho virtual, equação (4.23), toma a seguinte forma:

$$
\delta T=\int_{S} \int_{-e / 2}^{e / 2}\left\{\left[\frac{E}{\left(1-\nu^{2}\right)}\left(\frac{\partial \theta_{1}}{\partial x_{1}}+\nu \frac{\partial \theta_{2}}{\partial x_{2}}\right) \frac{\partial \overline{\theta_{1}}}{\partial x_{1}}\right] x_{3}^{2}+\left[\frac{E}{\left(1-\nu^{2}\right)}\left(\frac{\partial \theta_{2}}{\partial x_{1}}+\nu \frac{\partial \theta_{1}}{\partial x_{1}}\right) \frac{\partial \overline{\theta_{2}}}{x_{2}}\right] x_{3}^{2}+\right.
$$




$$
\begin{array}{r}
{\left[\frac{E}{2(1+\nu)}\left(\frac{\partial \theta_{1}}{\partial x_{2}}+\frac{\partial \theta_{2}}{\partial x_{1}}\right)\left(\frac{\partial \overline{\theta_{1}}}{\partial x_{2}}+\frac{\partial \overline{\theta_{2}}}{\partial x_{1}}\right)\right] x_{3}^{2}-\left[\frac{E}{2(1+\nu)}\left(-\theta_{1}+\frac{\partial \omega}{\partial x_{1}}\right)\left(-\overline{\theta_{1}}+\frac{\partial \bar{\omega}}{\partial x_{1}}-\right)\right]-} \\
\left.\left[\frac{E}{2(1+\nu)}\left(-\theta_{2}+\frac{\partial \omega}{\partial x_{2}}\right)\left(-\overline{\theta_{2}}+\frac{\partial \bar{\omega}}{\partial x_{2}}-\right)\right]\right\} d S d x_{3}
\end{array}
$$

onde $\bar{\omega}, \overline{\theta_{1}}$ e $\overline{\theta_{2}}$ são deslocamentos e rotações virtuais. Procedendo à integração ao longo da espessura e substituindo as equações (4.10), (4.11), (4.12) e (4.18), obtém-se:

$$
\begin{array}{r}
\delta T=\int_{S}\left[M_{11}\left(\frac{\partial \overline{\theta_{1}}}{\partial x_{1}}\right)+M_{22}\left(\frac{\partial \overline{\theta_{2}}}{\partial x_{2}}\right)+M_{12}\left(\frac{\partial \overline{\theta_{1}}}{\partial x_{1}}+\frac{\partial \overline{\theta_{2}}}{\partial x_{2}}\right)\right] d S+ \\
\int_{S}\left[Q_{1}\left(-\overline{\theta_{1}}+\frac{\partial \bar{\omega}}{\partial x_{1}}\right)+Q_{2}\left(-\overline{\theta_{2}}+\frac{\partial \bar{\omega}}{\partial x_{2}}\right)\right] d S
\end{array}
$$

Aplicando o teorema de Green as integrais que envolvem as primeiras derivadas dos deslocamentos e rotações virtuais, obtém-se:

$$
\begin{array}{r}
\delta T=\int_{S}\left[-\frac{\partial M_{11}}{\partial x_{1}} \overline{\theta_{1}}-\frac{\partial M_{22}}{\partial x_{2}} \overline{\theta_{2}}-\frac{\partial M_{12}}{\partial x_{2}} \overline{\theta_{1}}-\frac{\partial M_{12}}{\partial x_{1}} \overline{\theta_{2}}+Q_{1} \overline{\theta_{1}}+Q_{2} \overline{\theta_{2}}+\frac{\partial Q_{1}}{\partial x_{1}} \bar{\omega}+\frac{\partial Q_{2}}{\partial x_{2}} \bar{\omega}\right] d S+ \\
\int_{\Gamma}\left[M_{11} \overline{\theta_{1}} \cos (\theta)+M_{22} \overline{\theta_{2}} \sin (\theta)+M_{12} \overline{\theta_{1}} \sin (\theta)+M_{12} \overline{\theta_{2}} \cos (\theta)\right] d \Gamma- \\
\int_{\Gamma}\left[Q_{1} \bar{\omega} \cos (\theta)+Q_{2} \bar{\omega} \sin (\theta)\right] d \Gamma
\end{array}
$$

onde $\theta$ é usado para designar o ângulo da normal ao contorno, $\Gamma$, com a direção do contorno, como mostrado na Figura 4.5.

Tendo em conta que as rotações segundo $x_{1}$ e $x_{2}$ se relacionam com as rotações segundo a normal ao contorno $\theta_{n}$ e com as rotações segundo a tangente $\theta_{s}$ da seguinte forma:

$$
\theta_{1}=\theta_{n} \cos (\theta)+\theta_{s} \sin (\theta)
$$



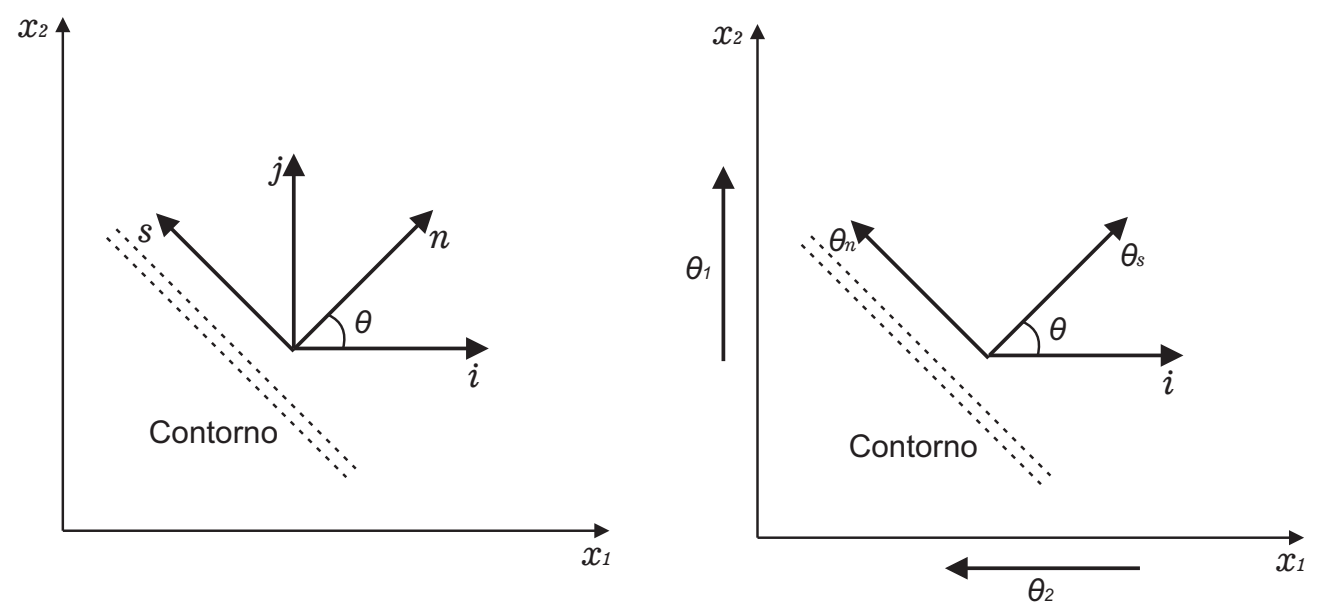

Figura 4.5: Rotações no contorno.

$$
\theta_{2}=\theta_{n} \sin (\theta)-\theta_{s} \cos (\theta)
$$

e que o momento normal, $M_{n}$, o momento tangente, $M_{s}$, e o esforço cortante, $Q$, no contorno, se relacionam com os esforços $M_{11}, M_{22}, M_{12}, Q_{1}$ e $Q_{2}$ da seguinte forma:

$$
\begin{gathered}
M_{n}(\theta)=M_{11} \cos ^{2}(\theta)+M_{22} \sin ^{2}(\theta)-2 M_{12} \sin (\theta) \cos (\theta) \\
M_{t}(\theta)=\left(M_{11}-M_{22}\right) \sin (\theta) \cos (\theta)-M_{12}\left(\cos ^{2}(\theta)-\sin ^{2}(\theta)\right) \\
Q(\theta)=Q_{1} \cos (\theta)+Q_{2} \sin (\theta)
\end{gathered}
$$

A equação (4.26) toma a forma:

$$
\begin{array}{r}
\delta T=\int_{S}\left[-\frac{\partial M_{11}}{\partial x_{1}} \overline{\theta_{1}}-\frac{\partial M_{22}}{\partial x_{2}} \overline{\theta_{2}}-\frac{\partial M_{12}}{\partial x_{2}} \overline{\theta_{1}}-\frac{\partial M_{12}}{\partial x_{1}} \overline{\theta_{2}}+Q_{1} \overline{\theta_{1}}+Q_{2} \overline{\theta_{2}}+\frac{\partial Q_{1}}{\partial x_{1}} \bar{\omega}+\frac{\partial Q_{2}}{\partial x_{2}} \bar{\omega}\right] d S+ \\
\int_{\Gamma}\left[M_{n} \overline{\theta_{n}}+M_{s} \overline{\theta_{n}}\right] d \Gamma-\int_{\Gamma} Q \bar{\omega} d \Gamma
\end{array}
$$


O trabalho realizado pelas forças externas, no caso da placa estar sujeita a uma distribuição de carga, $q$, normal ao plano médio da placa é, $\delta W$, calculado do seguinte modo a partir da carga e da deformação virtual:

$$
\delta W=-\int_{S} q \bar{\omega} d s
$$

O teorema dos trabalhos virtuais estabelece o equilíbrio do sistema, ou seja, a soma dos trabalhos internos e externos realizados deve ser nula. Dessa forma, tem-se:

$$
\delta T+\delta W=0
$$

Tendo em conta as equações (4.32) e (4.33) que definem o trabalho virtual dos esforços internos $\delta T$ e dos esforços externos $\delta W$, é possível obter três equações de equilíbrio que são coeficientes das rotações virtuais e dos deslocamentos virtuais nas integrais estendidas a superfície da placa e as condições de contorno representadas nas integrais estendidas ao contorno da placa. A energia potencial é definida a partir das energias de deformação interna e potencial externa, ou seja:

$$
\Pi=T+W
$$

sendo portanto definida de acordo com a expressão:

$$
\Pi=\frac{1}{2} \int_{V} \sigma_{i j} \varepsilon_{i j} d V-\int_{S} q d S
$$

ou seja, em termos dos esforços e deformações generalizadas:

$$
\begin{array}{r}
\Pi=\frac{1}{2} \int_{S}\left[M_{11}\left(\frac{\partial \theta_{1}}{\partial x_{1}}\right)+M_{22}\left(\frac{\partial \theta_{2}}{\partial x_{2}}\right)+M_{12}\left(\frac{\partial \theta_{1}}{\partial x_{2}}+\frac{\partial \theta_{2}}{\partial x_{1}}\right)\right] d S- \\
\frac{1}{2} \int_{S}\left[Q_{1}\left(-\theta_{1}+\frac{\partial \omega}{\partial x_{1}}\right)+Q_{2}\left(-\theta_{2}+\frac{\partial \omega}{\partial x_{2}}\right)\right] d S-
\end{array}
$$




$$
\int_{S} q \omega d S
$$

$\mathrm{Ou}$,

$$
\begin{array}{r}
\Pi=\frac{1}{2} \int_{S}\left(M_{11} \chi_{11}+M_{22} \chi_{22}+M_{12} \chi_{12}\right) d S- \\
-\frac{1}{2}\left(Q_{11} \chi_{1}+Q_{22}\right) d S-\int_{S} q \omega d S
\end{array}
$$

As equações de equilíbrio podem ser determinadas através da equação (4.38).

\subsection{Equações de equilíbrio}

As equações de equilíbrio podem ser formadas considerando o equilíbrio de um elemento de placa como mostrado na Figura 4.6. Este elemento de placa tem dimensões $d x_{1}, d x_{2}$ e $d x_{3}$ e está sujeito a um carregamento uniforme $q$ (por unidade de área), considerando positivo quando aplicado na direção de $x_{3}$. O equilíbrio dos momentos quando aplicado no eixo $x_{1}$ pode ser escrito como:

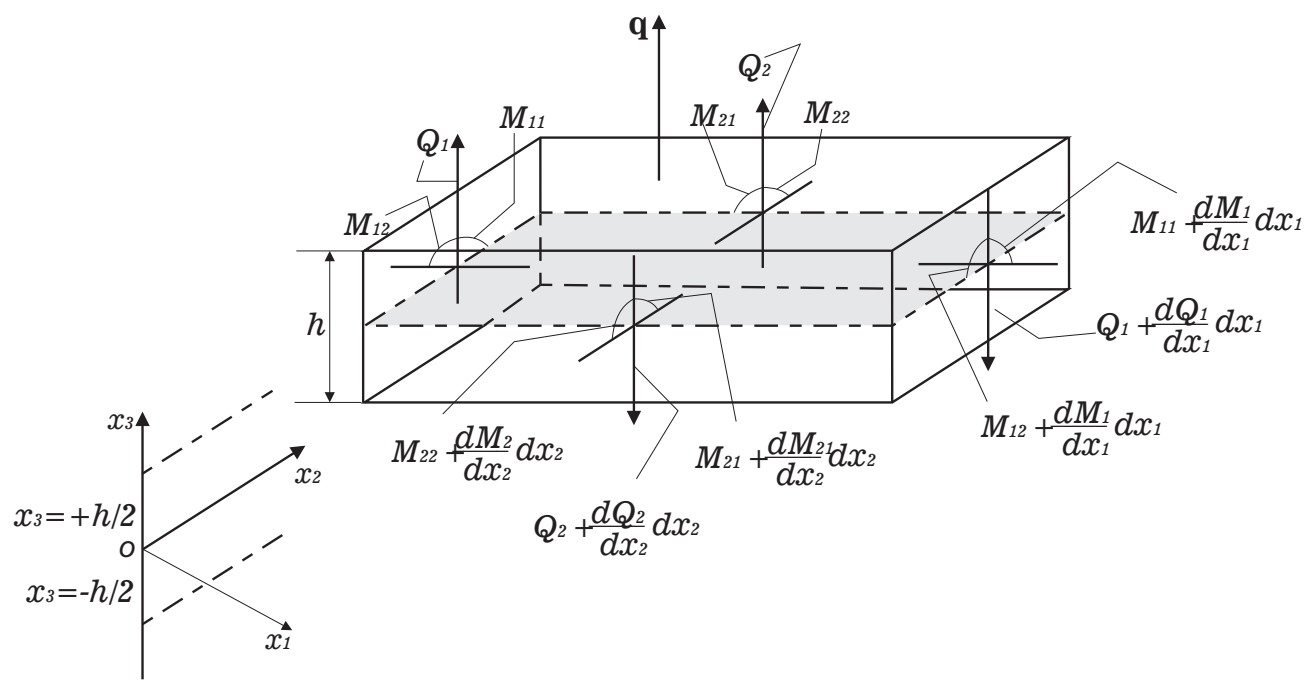

Figura 4.6: Equilíbrio em um elemento diferencial de placa. 


$$
\frac{\partial M_{22}}{\partial x_{2}}+\frac{\partial M_{12}}{\partial x_{1}}-Q_{2}=0
$$

De forma similar considera-se o equilíbrio do momento sobre o eixo $x_{2}$ é dado:

$$
\frac{\partial M_{11}}{\partial x_{1}}+\frac{\partial M_{12}}{\partial x_{2}}-Q_{1}=0
$$

O equilíbrio das forças na direção de $x_{3}$ pode ser escrito como:

$$
\frac{\partial Q_{1}}{\partial x_{1}}+\frac{\partial Q_{2}}{\partial x_{2}}+q=0
$$

Substituindo as equações (4.16) e (4.17) nas equações (4.39), (4.40) e (4.41), obtém-se:

$$
\begin{gathered}
-D\left(\frac{\partial^{2} \theta_{1}}{\partial x_{1}^{2}}+\frac{1-\nu}{2} \frac{\partial^{2} \theta_{1}}{\partial x_{2}^{2}}+\frac{1+\nu}{2} \frac{\partial^{2} \theta_{2}}{\partial x_{1} \partial x_{2}}\right)-G\left(\frac{\partial \omega}{\partial x_{1}}-\theta_{1}\right)=0 \\
-D\left(\frac{(1+\nu)}{2} \frac{\partial^{2} \theta_{1}}{\partial x_{1} \partial x_{2}}+\frac{(1-\nu)}{2} \frac{\partial^{2} \theta_{2}}{\partial x_{1}^{2}}+\frac{\partial^{2} \theta_{2}}{\partial x_{2}^{2}}\right)-G\left(\frac{\partial \omega}{\partial x_{2}}-\theta_{2}\right)=0 \\
G\left(\frac{\partial^{2} \omega}{\partial x_{1}^{2}}+\frac{\partial^{2} \omega}{\partial x_{2}^{2}}-\frac{\partial \theta_{1}}{\partial x_{1}}-\frac{\partial \theta_{2}}{\partial x_{2}}\right)-q\left(x_{1}, x_{2}\right)=0
\end{gathered}
$$

Estas são as equações de equilíbrio em termos dos deslocamentos transversais e das rotações. Nota-se que é necessário determinar através das equações (4.42), (4.43) e (4.44) o deslocamento transversal e as rotações. Através das equações (4.3), (4.4), (4.16) e (4.17) obtém-se os esforços cortantes e tensões. As equações (4.39), (4.40) e (4.41) representam a equação governante de placas na forma diferencial. Estas equações podem ser escritas em notação indicial como segue:

$$
M_{\alpha \beta, \beta}-Q_{\alpha}=0
$$




$$
Q_{\alpha, \alpha}+q=0
$$

Nota-se que as equações (4.45) e (4.46) possuem cinco termos desconhecidos, entretanto são apenas três equações. Dessa forma, sera necessária uma equação adicional para definir o problema através das relações de tensões $\times$ deslocamentos e aplicações de condições de contorno adequadas.

\subsection{Relações tensões $\times$ deslocamentos}

As tensões são derivadas usando-se o princípio básico da energia mínima para as tensões (Teorema de Castigliano), definindo a energia complementar П como segue (Rashed, 1999):

$$
\Pi=\Pi_{s}-\Pi_{b}
$$

onde $\Pi_{s}$ denota a energia de deformação e $\Pi_{b}$ denota o trabalho realizado ao longo do contorno. Para materiais elásticos isotrópicos, estes termos são dados por:

$$
\Pi_{s}=\frac{1}{2} \int_{\Omega}\left\{\frac{M_{\alpha \beta} M_{\alpha \beta}(1+\nu)-\nu M_{\alpha \alpha} M_{\theta \theta}}{\left(1-\nu^{2}\right) D}-\frac{2}{C_{n}} q M_{\alpha \alpha}+\frac{1}{C_{s}} Q_{\alpha} Q_{\alpha}\right\} d \Omega
$$

$\mathrm{e}$

$$
\Pi_{b}=\frac{1}{2} \int_{\Gamma}\left(M_{n} u_{n}+M_{t} u_{t}+Q_{n} u_{3}\right) d \Gamma
$$

onde

$$
\begin{gathered}
M_{n}=M_{\alpha \beta} n_{\beta} n_{\alpha} \\
M_{t}=M_{\alpha \beta} n_{\beta} t_{\alpha}
\end{gathered}
$$




$$
\begin{array}{r}
Q_{n}=p_{3} \\
u_{n}=u_{\gamma} n_{\gamma} \\
u_{t}=u_{\gamma} t_{\gamma}
\end{array}
$$

$M_{n}, M_{t}, Q_{n}, u_{n}$ e $u_{t}$, denotam respectivamente, o momento normal, o momento tangencial, o esforço cortante, o deslocamento normal e o deslocamento tangencial em pontos do contorno $\Gamma, t_{\alpha}$ denota a componente do vetor tangente em um ponto no contorno e $\nu$ denota o coeficiente de Poisson. As constantes $D, C_{n}$ e $C_{s}$ são dependentes das propriedades do material (conforme a equação (4.48)). Pode-se notar na equação (4.48) que o primeiro termo no lado direito representa a contribuição da deformação de flexão à energia de deformação. O segundo termo representa o efeito da tensão normal transversal na energia de deformação e o último termo é a contribuição da deformação de cisalhamento à energia de deformação. Este último termo representa a principal diferença entre a teoria clássica de placas (Kirchhoff, 1950) e a teoria de placas deformadas por cisalhamento (Mindlin, 1951).

Afim de obter a mínima energia nas equações (4.45) e (4.46), estas equações tem de ser ponderadas por $u_{i}$ e integradas sobre o domínio $\Omega$. Dessa forma, a variação da soma integral das equações (4.45) e (4.46) com a energia complementar será definido como zero, isto é:

$$
\delta\left[\Pi+\int_{\Omega}\left(M_{\alpha \beta, \beta}-Q_{\alpha}\right) u_{\alpha}+\left(Q_{\alpha, \alpha}+q\right) u_{3} d \Omega\right]=0
$$

onde $\delta[$.$] denota o operador variacional delta (Dym, 1973). Integrando por partes e despre-$ zando as variações das derivadas, tem-se:

$$
\begin{array}{r}
\int_{\Omega}\left\{\left[\frac{M_{\alpha \beta}(1+\nu)-\nu M_{\theta \theta} \delta_{\alpha \beta}}{\left(1-\nu^{2}\right) D}-\frac{q}{C_{n}} \delta_{\alpha \beta}-u_{\alpha, \beta}\right] \delta\left[M_{\alpha \beta}\right]\right. \\
\left.+\left[\frac{Q_{\alpha}}{C_{s}}-u_{3, \alpha}-u_{\alpha}\right] \delta\left[Q_{\alpha}\right]\right\} d \Omega=0
\end{array}
$$

que implica em: 


$$
\frac{M_{\alpha \beta}(1+\nu)-\nu M_{\theta \theta} \delta_{\alpha \beta}}{\left(1-\nu^{2}\right) D}-\frac{q}{C_{n}} \delta_{\alpha \beta}-u_{\alpha, \beta}=0
$$

e

$$
\frac{Q_{\alpha}}{C_{s}}-u_{3, \alpha}-u_{\alpha}=0
$$

$\mathrm{ou}$,

$$
M_{\alpha \beta}=D \frac{1-\nu}{2}\left(2 \chi_{\alpha \beta}+\frac{2 \nu}{(1-\nu)} \chi_{\gamma \gamma} \delta_{\alpha \beta}\right)+D \frac{(1+\nu)}{C_{n}} q \delta_{\alpha \beta}
$$

$\mathrm{e}$

$$
Q_{\alpha}=C_{s} \psi_{\alpha}
$$

onde

$$
\chi_{\alpha \beta}=\frac{1}{2}\left(u_{\alpha, \beta}+u_{\beta, \alpha}\right)
$$

e

$$
\psi_{\alpha}=u_{\alpha}+u_{3, \alpha}
$$

sendo $\delta_{\alpha \beta}$ é a função delta de Kronecker, $\chi_{\alpha \beta}$ denota a deformação por flexão e $\psi_{\alpha}$ denota a deformação por cisalhamento. As equações (4.55) e (4.56) representam a lei de Hooke generalizada. As Equações (4.55) e (4.56) juntas com as equações (4.57) e (4.58) representam a relações entre as tensões $\times$ deslocamentos generalizados. 


\subsection{Condições de contorno}

Após a criação das relações tensão $\times$ deslocamentos, o problema é definido em termos dos valores das condições de contorno, $u_{i}$ e $p_{i}$. Os possíveis tipos de condições de contorno podem ser resumidas como segue:

\subsubsection{Borda apoiada}

A condição de borda apoiada o movimento segundo o eixo $O x_{3}$ está impedido, podendo no entanto girar livremente, Figura 4.7. As condições de contorno apoiada são:

$$
u_{3}=0 \Longleftrightarrow u_{t}=0 \Longleftrightarrow M_{n}=M_{\text {aplicado }}
$$

sendo $u_{3}$ o deslocamento transversal, $u_{t}$ a rotação tangente e $M_{n}$ o momento que provoca uma rotação normal no bordo simplesmente apoiado. As condições de contorno da placa mostrada são:

1. Ao longo dos lados $A B$ e $C D$ que correspondem a $x_{1}=0$ e $x_{1}=a$ :

$$
u_{3}=0 \Longleftrightarrow u_{t}=0 \Longleftrightarrow M_{n}=0
$$

2. Ao longo dos lados $A C$ e $B D$ que correspondem a $x_{2}=0$ e $x_{2}=b$ :

$$
u_{3}=0 \Longleftrightarrow u_{t}=0 \Longleftrightarrow M_{n}=0
$$

\subsubsection{Borda engastada}

A condição de borda engastada segundo o eixo $O x_{3}$ está impedindo deslocamentos e rotações, Figura 4.8. As condições de contorno engastada são:

$$
u_{3}=0 \Longleftrightarrow u_{t}=0 \Longleftrightarrow u_{n}=0
$$




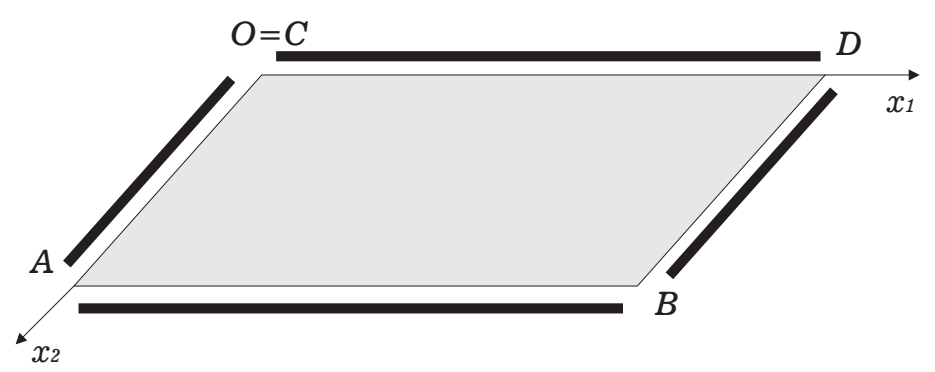

Figura 4.7: Placa com borda apoiada.

sendo $u_{3}$ o deslocamento transversal, $u_{t}$ a rotação tangente e $u_{n}$ a rotação normal. As condições de contorno da placa mostrada são:

1. Ao longo dos lados $A B$ e $C D$ que correspondem a $x_{1}=0$ e $x_{1}=a$ :

$$
u_{3}=0 \Longleftrightarrow u_{t}=0 \Longleftrightarrow u_{n}=0
$$

2. Ao longo dos lados $A C$ e $B D$ que correspondem a $x_{2}=0$ e $x_{2}=b$ :

$$
u_{3}=0 \Longleftrightarrow u_{t}=0 \Longleftrightarrow u_{n}=0
$$

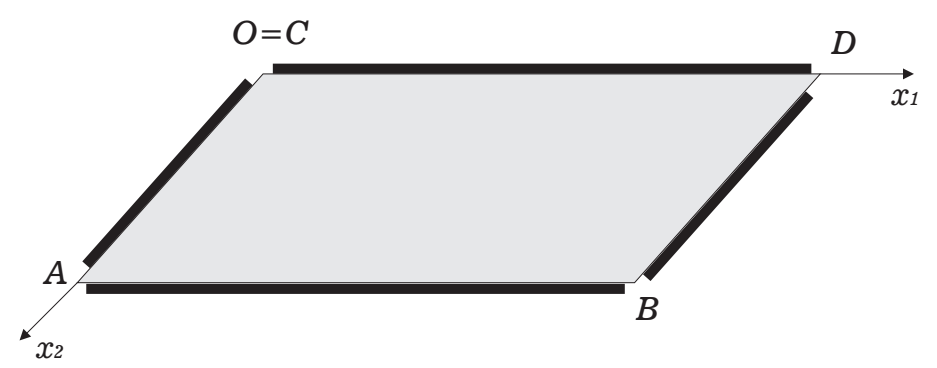

Figura 4.8: Placa com borda engastada.

\subsubsection{Borda livre}

Se a placa tiver um ou mais lados livres, em todos os pontos do bordo livre os momentos fletores $M_{n}$ e $M_{t}$ e os esforços transversais $Q$ devem ser nulos. 


\subsection{Avaliação das constantes}

Afim de calcular os valores das constantes $D, C_{n}$ e $C_{s}$, a energia de deformação na equação (4.48) será igual a energia de deformação liberada da placa quando é tratada como uma estrutura tri-dimensional (Timoshenko e Goodier, 1934). A energia de deformação é dada por:

$$
\begin{aligned}
\Pi_{s}= & \frac{1}{2} \int_{\Omega} \int_{-h / 2}^{h / 2}\left\{\frac{1}{E}\left(\sigma_{\alpha \beta} \sigma_{\alpha \beta}(1+\nu)-\nu \sigma_{\alpha \alpha} \sigma_{\theta \theta}\right)\right. \\
& \left.+\frac{1}{E}\left(\sigma_{33}-2 \nu \sigma_{\alpha \alpha}\right) \sigma_{33}+\frac{1}{G} \sigma_{\alpha 3} \sigma_{\alpha 3}\right\} d x_{3} d \Omega
\end{aligned}
$$

onde $E$ é o módulo de elasticidade e $G$ é o módulo de cisalhamento. Afim de comparar as equações (4.48) e (4.59), uma variação apropriada de tensões através da espessura será assumida. Reissner (1947) propôs que as tensões devido à flexão variam linearmente ao longo da espessura e as tensões devido os esforços cortantes variam parabolicamente através da espessura, através das seguintes relações:

$$
\begin{gathered}
\sigma_{\alpha \beta}=\frac{12 x_{3}}{h^{3}} M_{\alpha \beta} \\
\sigma_{3 \alpha}=\frac{3}{2 h}\left[1-\left(\frac{2 x_{3}}{h}\right)^{2}\right] Q_{\alpha}
\end{gathered}
$$

Estas relações satisfazem a equação diferencial tridimensional de equilíbrio desde que o momento fletor e a força cortante satisfaçam as equações (4.45) e (4.46). Da consideração de equilíbrio em três dimensões e considerando que a carga $q$ é aplicada em $x_{3}= \pm h / 2$, a tensão normal $\sigma_{33}$ pode ser obtida como segue:

$$
\sigma_{33}=\frac{1}{4} \frac{2 x_{3}}{h}\left[3-\left(\frac{2 x_{3}}{h}\right)^{2}\right] q
$$


Substituindo as equações (4.60) e (4.61) na equação (4.59), então integrando com relação a $x_{3}$ e comparando com a equação (4.48), os valores das constantes $D, C_{n}$ e $C_{s}$ são:

$$
\begin{gathered}
D=\frac{E h^{3}}{12\left(1-\nu^{2}\right)} \\
C_{n}=\frac{5 E h}{6 \nu} \\
C_{s}=\frac{5}{6} G h
\end{gathered}
$$

Substituindo os valores das constantes $D, C_{n}$ e $C_{s}$ nas equações (4.55) e (4.56), obtémse:

$$
\begin{gathered}
M_{\alpha \beta}=D \frac{1-\nu}{2}\left(u_{\alpha, \beta}+u_{\beta, \alpha}+\frac{2 \nu}{1-\nu} u_{\gamma, \gamma} \delta_{\alpha \beta}\right)+\frac{\nu q}{(1-\nu) \lambda^{2}} \delta_{\alpha \beta} \\
Q_{\alpha}=D \frac{1-\nu}{2} \lambda^{2}\left(u_{\alpha}+u_{3, \alpha}\right)
\end{gathered}
$$

As equações (4.66) e (4.67) representam as relações entre tensões $\times$ deslocamentos generalizados, onde $\lambda=\sqrt{10} / h$ é o fator cisalhante.

\subsection{Equação de Navier}

A equação generalizada de Navier pode ser formada por substituição dos valores das constantes nas equações (4.45) e (4.46):

$$
L_{i j} u_{j}+b_{i}=0
$$

em que, 


$$
\begin{gathered}
L_{\alpha \beta}=D \frac{(1-\nu)}{2}\left[\left(\nabla^{2}-\lambda^{2}\right) \delta_{\alpha \beta}+\frac{1+\nu}{1-\nu} \partial_{\alpha} \partial_{\beta}\right] \\
L_{\alpha 3}=-C \partial_{\alpha} \\
L_{3 \beta}=C \partial_{\beta} \\
L_{33}=C \nabla^{2}
\end{gathered}
$$

e

$$
C=D\left(\frac{1-\nu}{2}\right) \lambda^{2}
$$

onde $L_{i j}$ é o operador diferencial de Navier, $\nabla^{2}$ é o operador laplaciano, e $b_{i}$ é uma força de corpo generalizada que é dada por (Rashed, 1999):

$$
\begin{gathered}
b_{\alpha}=\frac{\nu}{(1-\nu) \lambda^{2}} q_{, \alpha} \\
b_{3}=q
\end{gathered}
$$

Substituindo as equações (4.69), (4.70), (4.71) e (4.72) na equação (4.68):

$$
\begin{aligned}
& D \nabla^{2} w_{1}+\frac{D}{2}(1+\nu) \frac{\partial}{\partial x_{2}}\left(-\frac{\partial w_{1}}{\partial x_{2}}+\frac{\partial w_{2}}{\partial x_{1}}\right)-C w_{1}-C \frac{\partial w_{3}}{\partial x_{1}}=0 \\
& \frac{D}{2}(1+\nu) \frac{\partial}{\partial x_{1}}\left(-\frac{\partial w_{1}}{\partial x_{2}}+\frac{\partial w_{2}}{\partial x_{1}}\right)+D \nabla^{2} w_{2}-C w_{2}-C \frac{\partial w_{3}}{\partial x_{2}}=0
\end{aligned}
$$




$$
\begin{gathered}
C \nabla^{2} w_{3}+C \frac{\partial w_{1}}{\partial x_{1}}+C \frac{\partial w_{2}}{\partial x_{2}}+q_{3}=0 \\
B \nabla^{2} u_{1}+\frac{B}{2}(1+\nu) \frac{\partial}{\partial x_{2}}\left(-\frac{\partial u_{1}}{\partial x_{2}}+\frac{\partial u_{2}}{\partial x_{1}}\right)+q_{1}=0
\end{gathered}
$$




\section{Capítulo 5}

\section{Método dos elementos de contorno para placas isotrópicas}

\subsection{Introdução}

Neste Capítulo é desenvolvida a formulação do método dos elementos de contorno para placas isotrópicas. A formulação isotrópica é desenvolvida com base na teoria de reciprocidade de Betti, os kernels presentes na solução fundamental isotrópica são dados por Vander Weeën (1982). As integrais de domínio provenientes das forças de corpo são transformadas em integrais de contorno usando o método dos elementos de contorno de integração radial (MIR).

\subsection{Representação integral}

A equação integral pode ser obtida através da representação das equações (4.45) e (4.46) através da seguinte identidade integral (Rashed, 1999):

$$
\int_{\Omega}\left[\left(M_{\alpha \beta, \beta}-Q_{\alpha}\right) U_{\alpha}^{*}+\left(Q_{\alpha, \alpha}+q\right) U_{3}^{*}\right] d \Omega=0
$$


onde, $U_{i}^{*}\left(i=\alpha^{1}, 3\right)$ são funções ponderadoras. Expandindo a equação (5.1), tem-se:

$$
\int_{\Omega} U_{\alpha}^{*} M_{\alpha \beta, \beta} d \Omega-\int_{\Omega} U_{\alpha}^{*} Q_{\alpha} d \Omega+\int_{\Omega} U_{3}^{*} Q_{\alpha, \alpha} d \Omega+\int_{\Omega} U_{3}^{*} q d \Omega=0
$$

A segunda identidade de Green é aplicada no primeiro e segundo termo no lado esquerdo da equação (5.2), tem-se:

$$
\int_{\Omega} U_{\alpha}^{*} M_{\alpha \beta, \beta} d \Omega=\int_{\Gamma} U_{\alpha}^{*} M_{\alpha \beta} n_{\beta} d \Gamma-\int_{\Omega} M_{\alpha \beta} U_{\alpha, \beta}^{*} d \Omega
$$

Fazendo $p_{\alpha}=M_{\alpha \beta} n_{\beta}$, tem-se:

$$
\int_{\Omega} U_{\alpha}^{*} M_{\alpha \beta, \beta} d \Omega=\int_{\Gamma} U_{\alpha}^{*} p_{\alpha} d \Gamma-\int_{\Omega} M_{\alpha \beta} U_{\alpha, \beta}^{*} d \Omega
$$

Da mesma forma, o terceiro termo da equação (5.2) pode ser escrito como:

$$
\int_{\Omega} U_{3}^{*} Q_{\alpha, \alpha} d \Omega=\int_{\Gamma} U_{3}^{*} Q_{\alpha} n_{\alpha} d \Gamma-\int_{\Omega} Q_{\alpha} U_{3, \alpha}^{*} d \Omega
$$

Fazendo $p_{3}=Q_{\alpha} n_{\alpha}$, tem-se:

$$
\int_{\Omega} U_{3}^{*} Q_{\alpha, \alpha} d \Omega=\int_{\Gamma} U_{3}^{*} p_{3} d \Gamma-\int_{\Omega} Q_{\alpha} U_{3, \alpha}^{*} d \Omega
$$

Substituindo as equações (5.4) e (5.6) na equação (5.1), tem-se:

$$
\int_{\Gamma} U_{\alpha}^{*} p_{\alpha} d \Gamma-\int_{\Omega} M_{\alpha \beta} U_{\alpha, \beta}^{*} d \Omega-\int_{\Omega} Q_{\alpha} U_{\alpha}^{*} d \Omega+\int_{\Gamma} U_{3}^{*} p_{3} d \Gamma-\int_{\Omega} Q_{\alpha} U_{3, \alpha}^{*} d \Omega+\int_{\Omega} q U_{3}^{*} d \Omega=0
$$

A equação (5.7) toma a seguinte forma:

$$
{ }^{1} \alpha=1,2
$$




$$
\int_{\Gamma} U_{\alpha}^{*} p_{\alpha} d \Gamma+\int_{\Gamma} U_{3}^{*} p_{3} d \Gamma+\int_{\Omega} q U_{3}^{*} d \Omega-\left[\int_{\Omega} Q_{\alpha} U_{\alpha}^{*} d \Omega+\int_{\Omega} U_{3, \alpha}^{*} Q_{\alpha} d \Omega\right]-\int_{\Omega} M_{\alpha \beta} U_{\alpha, \beta}^{*} d \Omega=0
$$

Os dois primeiros termos do lado direito da equação (5.8) podem ser substituídos pela seguinte notação:

$$
\int_{\Gamma} U_{\alpha}^{*} p_{\alpha} d \Gamma+\int_{\Gamma} U_{3}^{*} p_{3} d \Gamma=\int_{\Gamma} U_{j}^{*} p_{j} d \Gamma
$$

onde, $j=1,3$. Dessa forma, tem-se:

$$
\int_{\Gamma} p_{j} U_{j}^{*} d \Gamma+\int_{\Omega} q U_{3}^{*} d \Omega+\int_{\Omega} Q_{\alpha}\left(U_{\alpha}^{*}+U_{3, \alpha}^{*}\right) d \Omega+\int_{\Omega} M_{\alpha \beta} U_{\alpha, \beta}^{*} d \Omega=0
$$

Substituindo os esforços resultantes $\left(M_{\alpha \beta}\right.$ e $\left.Q_{\alpha}\right)$ com os deslocamentos generalizados e suas derivadas usando as equações (4.66) e (4.67), e então aplicando a segunda identidade de Green para a integral $M_{\alpha \beta}$, obtém-se:

$$
\begin{array}{r}
\int_{\Gamma} p_{j} U_{j}^{*} d \Gamma+\int_{\Omega} Q_{\alpha}^{*}\left(u_{\alpha}+\alpha_{3, \alpha}\right) d \Omega \\
-\int_{\Gamma} U_{\alpha, \beta}^{*}\left\{\frac{D(1-\nu)}{2}\left[u_{\alpha} n_{\beta}+u_{\beta} n_{\alpha}+\frac{2 \nu}{(1-\nu)} u_{\gamma} n_{\gamma} \delta_{\alpha \beta}\right]\right\} d \Gamma \\
-\int_{\Gamma} \frac{D(1-\nu)}{2}\left[u_{\alpha} U_{\alpha, \beta \beta}^{*}+u_{\beta} U_{\alpha, \beta \alpha}^{*}+\frac{2 \nu}{(1-\nu)} u_{\gamma} U_{\alpha, \beta \gamma}^{*} \delta_{\alpha \beta}\right] d \Gamma \\
+\int_{\Omega}\left[q U_{3}^{*}+\frac{\nu q}{(1-\nu) \lambda^{2}} \delta_{\alpha \beta} U_{\alpha, \beta}^{*}\right] d \Omega=0
\end{array}
$$

O segundo termo do lado esquerdo da equação (5.11) pode ser decomposto usando a segunda identidade de Green e fazendo o uso das relações das equações (4.21) e (4.22), tem-se:

$$
\begin{array}{r}
\int_{\Omega} Q_{\alpha}\left(u_{\alpha}+u_{3, \alpha}\right) d \Omega=\int_{\Omega} Q_{\alpha}^{*} u_{\alpha} d \Omega+\int_{\Omega} Q_{\alpha}^{*} u_{3, \alpha} d \Omega \\
=\int_{\Omega} Q_{\alpha}^{*} u_{\alpha} d \Omega+\int_{\Gamma} Q_{\alpha}^{*} u_{3} n_{\alpha} d \Gamma-\int_{\Omega} Q_{\alpha, \alpha}^{*} u_{3} d \Omega \\
=\int_{\Gamma} p_{3} u_{3} d \Gamma+\int_{\Omega}\left(Q_{\alpha}^{*} u_{\alpha}-Q_{\alpha, \alpha}^{*} u_{3}\right) d \Omega
\end{array}
$$


Substituindo a equação (5.12) na (5.11) e agrupando, tem-se:

$$
\begin{array}{r}
\int_{\Gamma}\left(U_{j}^{*} p_{j}-P_{j}^{*} u_{j}\right) d \Gamma+\int_{\Omega}\left[U_{3}^{*}+\frac{\nu}{(1-\nu) \lambda^{2}} U_{\theta, \theta}^{*}\right] d \Omega \\
+\int_{\Omega}\left[\left(M_{\alpha \beta, \beta}^{*}-Q_{\alpha}^{*}\right) u_{\alpha}+Q_{\alpha, \alpha}^{*} u_{\alpha}\right] d \Omega=0
\end{array}
$$

A equação (5.13) representa o teorema da reciprocidade de Betti para placas de Reissner. O estado $(\cdot)^{*}$ é escolhido arbitrariamente. Este estado é definido para cargas generalizadas concentradas: dois momentos de flexão $(i=\alpha=1,2)$ e uma força cisalhante centrada $(i=3)$ em um ponto arbitrário $X^{\prime} \in \Omega$.

$$
\begin{array}{r}
\int_{\Gamma}\left(U_{i j}^{*}\left(X^{\prime}, x\right) p_{j}(x)-P_{i j}^{*}\left(X^{\prime}, x\right) u_{j}(x)\right) d \Gamma(x) \\
+\int_{\Omega}\left[U_{i, \theta}^{*}\left(X^{\prime}, X\right)+\frac{\nu}{(1-\nu) \lambda^{2}} U_{i \theta, \theta}^{*}\left(X^{\prime}, X\right)\right] q d \Omega(X) \\
\left.+\int_{\Omega}\left[M_{i \alpha \beta, \beta}^{*}\left(X^{\prime}, X\right)-Q_{i \alpha}^{*}\left(X^{\prime}, X\right)\right] u_{\alpha}(X) d \Omega(X)\right] \\
+\int_{\Omega} Q_{i \alpha, \alpha}^{*}\left(X^{\prime}, X\right) u_{3}(X) d \Omega(X)=0
\end{array}
$$

onde $x \in \Gamma$ e $X^{\prime} \in \Omega$ são pontos campo no contorno e dentro do domínio, respectivamente. A escolha do estado fundamental $(\cdot)^{*}$ é dado por:

$$
\begin{gathered}
M_{i \alpha \beta, \beta}^{*}\left(X^{\prime}, X\right)-Q_{i \alpha}^{*}=-\delta\left(X^{\prime}, X\right) \delta_{i \alpha} \\
Q_{i \alpha, \alpha}^{*}\left(X^{\prime}, X\right)=-\delta\left(X^{\prime}, X\right) \delta_{i 3}
\end{gathered}
$$

onde $\delta\left(X^{\prime}, X\right)$ é o delta de Dirac. Fazendo o uso das seguintes propriedades (Greenberg, 1971): 


$$
\int_{\Omega} \delta\left(X^{\prime}, X\right) u_{i}(X) d \Omega=u_{i}\left(X^{\prime}\right)
$$

Então a equação (5.14) pode ser escrita para um ponto interno $X^{\prime}$ como:

$$
\begin{array}{r}
u_{j}\left(X^{\prime}\right)+\int_{\Gamma} P_{i j}^{*}\left(X^{\prime}, x\right) u_{j}(x) d \Gamma(x)=\int_{\Gamma} U_{i j}^{*}\left(X^{\prime}, x\right) p_{j}(x) d \Gamma(x) \\
\quad+\int_{\Omega}\left(U_{i 3}^{*}\left(X^{\prime}, X\right)-\frac{\nu}{(1-\nu) \lambda^{2}} U_{i \alpha, \alpha}^{*}\left(X^{\prime}, X\right)\right) q(X) d \Omega(X)
\end{array}
$$

onde $U_{i j}^{*}\left(X^{\prime}, x\right)$ e $P_{i j}^{*}\left(X^{\prime}, x\right)$ são as soluções fundamentais para deslocamentos e esforços generalizados, respectivamente. Estes representam os deslocamentos ou esforços no ponto $x$ ou $X$ na direção $j$ devido uma carga unitária aplicada em $X^{\prime}$ na direção $i$. As expressões para estas soluções fundamentais são dadas por Vander Weeën (1982) como segue:

$$
\begin{gathered}
U_{\alpha \beta}^{*}=\frac{1}{8 \pi D(1-\nu)}\left\{[8 B(z)-(1-\nu)(2 \log (z)-1)] \delta_{\alpha \beta}-[8 A(z)+2(1-\nu)] r_{, \alpha} r_{, \beta}\right\} \\
U_{\alpha, 3}^{*}=-U_{3 \alpha}^{*}=\frac{1}{8 \pi D}(2 \log (z)-1) r r_{, \alpha} \\
U_{33}^{*}=\frac{1}{8 \pi D(1-\nu) \lambda^{2}}\left[(1-\nu) z^{2}(\log (z)-1)-8 \log (z)\right]
\end{gathered}
$$

e

$$
\begin{array}{r}
P_{\gamma \alpha}^{*}=\frac{-1}{4 \pi r}\left[\left(4 A(z)+2 z K_{1}(z)+1-\nu\right)\left(\delta_{\alpha \gamma} r_{, n}+r_{, \alpha} n_{\gamma}\right)+(4 A(z)+1+\nu) r_{\gamma} n_{\alpha}\right. \\
\left.-2\left(8 A(z)+2 z K_{1}(z)+1-\nu\right) r_{, \alpha} r_{, \gamma} r_{, n}\right] \\
P_{\gamma 3}^{*}=\frac{\lambda^{2}}{2 \pi}\left[B(z) n_{\gamma}-A(z) r_{, \gamma} r_{, n}\right]
\end{array}
$$




$$
\begin{gathered}
P_{3 \alpha}^{*}=-\frac{(1-\nu)}{8 \pi}\left[\left(2 \frac{(1+\nu)}{(1-\nu)} \log (z)-1\right) n_{\alpha}+2 r_{, \alpha} r_{, n}\right] \\
P_{33}^{*}=\frac{-1}{2 \pi r} r_{, n}
\end{gathered}
$$

onde

$$
\begin{aligned}
& A(z)=K_{o}(z)+\frac{2}{z}\left[K_{1}(z)-\frac{1}{z}\right] \\
& B(z)=K_{o}(z)+\frac{1}{z}\left[K_{1}(z)-\frac{1}{z}\right]
\end{aligned}
$$

em que $K_{o}(z)$ e $K_{1}(z)$ são funções de Bessel (Abramowitz, 1965), $z=\lambda r, \lambda$ é o fator cisalhante, $r$ é a distância absoluta entre o ponto fonte e campo, $r_{, \alpha}=r_{\alpha} / r$ onde $r_{\alpha}=$ $x_{\alpha}(x)-x_{\alpha}\left(x^{\prime}\right)$ e $r_{, n}=r_{, \alpha} n_{\alpha}$, onde é $n$ é uma direção específica. A equação (5.18) representa a identidade de Somigliana para placas de Reissner. Expandindo as funções de Bessel para pequenos argumentos, tem-se:

$$
\begin{gathered}
K_{o}(z)=\left[-\gamma-\log \left(\frac{z}{2}\right)\right]+\left[-\gamma+1-\log \left(\frac{z}{2}\right)\right] \frac{\left(z^{2} / 4\right)}{(1 !)^{2}} \\
+\left[-\gamma+1+\frac{1}{2}-\log \left(\frac{z}{2}\right)\right] \frac{\left(z^{2} / 4\right)^{2}}{(2 !)^{2}} \\
+\left[-\gamma+1+\frac{1}{2}+\frac{1}{3}-\log \left(\frac{z}{2}\right)\right] \frac{\left(z^{2} / 4\right)^{3}}{(3 !)^{2}}+\ldots \\
K_{1}(z)=\frac{1}{z}-\left[-\gamma+\frac{1}{2}-\log \left(\frac{z}{2}\right)\right] \frac{\left(z^{2} / 4\right)^{1 / 2}}{(0 ! 1 !)} \\
-\left[-\gamma+1+\frac{1}{4}-\log \left(\frac{z}{2}\right)\right] \frac{\left(z^{2} / 4\right)^{3 / 2}}{(1 ! 2 !)} \\
-\left[-\gamma+1+\frac{1}{2}+\frac{1}{6}-\log \left(\frac{z}{2}\right)\right] \frac{\left(z^{2} / 4\right)^{5 / 2}}{(2 ! 3 !)}+\ldots
\end{gathered}
$$


onde, $\gamma=0,5772156649$ é a constante de Euler. Substituindo as equações (5.28) e (5.29) nas equações (5.26) e (5.27), respectivamente, e fazendo o limite $r \rightarrow 0$, tem-se:

$$
\begin{gathered}
\lim _{r \rightarrow 0} A(z)=-\frac{1}{2} \\
\lim _{r \rightarrow 0} B(z)=-\frac{1}{2}\left[\lim _{r \rightarrow 0} \log \left(\frac{z}{2}\right)+\gamma+\frac{1}{2}\right]
\end{gathered}
$$

Como pode ser visto, $A(z)$ é uma função suave; por outro lado, $B(z)$ apresenta uma singularidade fraca $\log (r)$. Portanto, $U_{i j}^{*}$ apresenta singularidade fraca e $P_{i j}^{*}$ tem uma singularidade forte $1 / r$.

\subsection{Equação integral de contorno}

Tomando o ponto $X^{\prime}$ no contorno e a posição $x^{\prime} \in \Gamma$ (ver Figura 4.1), e assumindo que os deslocamentos $u_{j}$ satisfazem a continuidade de Hölder, a equação (5.18) pode ser escrita como:

$$
\begin{array}{r}
c_{i j} u_{j}\left(x^{\prime}\right)+f_{\Gamma} P_{i j}^{*}\left(x^{\prime}, x\right) u_{j}(x) d \Gamma(x)=\int_{\Gamma} U_{i j}^{*}\left(x^{\prime}, x\right) p_{j}(x) d \Gamma(x) \\
+\int_{\Omega}\left[U_{i 3}^{*}\left(x^{\prime}, X\right)-\frac{\nu}{(1-\nu) \lambda^{2}} U_{i \alpha, \alpha}^{*}\left(x^{\prime}, X\right)\right] q(X) d \Omega(X)
\end{array}
$$

onde $f_{\Gamma}$ denota o valor da integral no sentido do valor principal de Cauchy, $x^{\prime}, x \in \Gamma$ são pontos fonte e campo, respectivamente, e $c_{i j}\left(x^{\prime}\right)$ depende da posição do ponto fonte ser interna, externa ou no contorno. Quando $x^{\prime}$ está localizado em uma região suave do contorno $\Gamma$ seu valor é igual a $1 / 2 \delta_{i j}$ (Barcelos e Westphal, 1992). A equação (5.32) representa três equações integrais: duas $(i=\alpha=1,2)$ para rotação e uma $(i=3)$ para deflexão. Como pode ser visto, a equação (5.32) é escrita em termos de deslocamentos generalizados. A integral de domínio na equação (5.32) pode ser transformada para integral de contorno através da aplicação do teorema da divergência da seguinte forma (Greenberg, 1977): 


$$
\begin{aligned}
& \int_{\Omega}\left[U_{i 3}^{*}\left(x^{\prime}, X\right)-\frac{\nu}{(1-\nu) \lambda^{2}} U_{i \alpha, \alpha}^{*}\left(x^{\prime}, X\right)\right] q(X) d \Omega(X) \\
= & \int_{\Gamma}\left[V_{i, \alpha}^{*}\left(x^{\prime}, x\right)-\frac{\nu}{(1-\nu) \lambda^{2}} U_{i \alpha}^{*}\left(x^{\prime}, x\right)\right] q(X) n_{\alpha}(x) d \Gamma(x)
\end{aligned}
$$

onde $V_{i}^{*}$ é a solução particular da equação $V_{i, \theta \theta}^{*}=U_{i 3}^{*}$. As expressões para $V_{i, \beta}^{*}$ são dadas por:

$$
\begin{gathered}
V_{\alpha, \beta}^{*}=\frac{r^{2}}{128 \pi D}\left[(4 \log (z)-5) \delta_{\alpha \beta}+2(4 \log (z)-3) r_{, \alpha} r_{, \beta}\right] \\
V_{3, \beta}^{*}=\frac{-r r_{, \beta}}{128 \pi D(1-\nu) \lambda^{2}}\left[32(2 \log (z)-1)-z^{2}(1-\nu)(4 \log (z)-5)\right]
\end{gathered}
$$

\subsection{Discretização}

A solução analítica da equação (5.32) é difícil de obter mesmo para um simples problema de placa. Portanto, a solução numérica pode ser considerada como uma alternativa. Dessa forma, o contorno $\Gamma$ tem de ser discretizado em $N_{e}$ elementos, sobre o qual as incógnitas são aproximadas para variar constantemente se os elementos são constantes. Estes elementos são chamados elementos de contorno.

\subsubsection{Elementos constantes}

Considere a Figura 5.1 onde o contorno de uma placa é aproximado por uma série de segmentos (elementos de contorno) $\Gamma_{j}$, cujo número e forma são escolhidos para representá-lo adequadamente. 


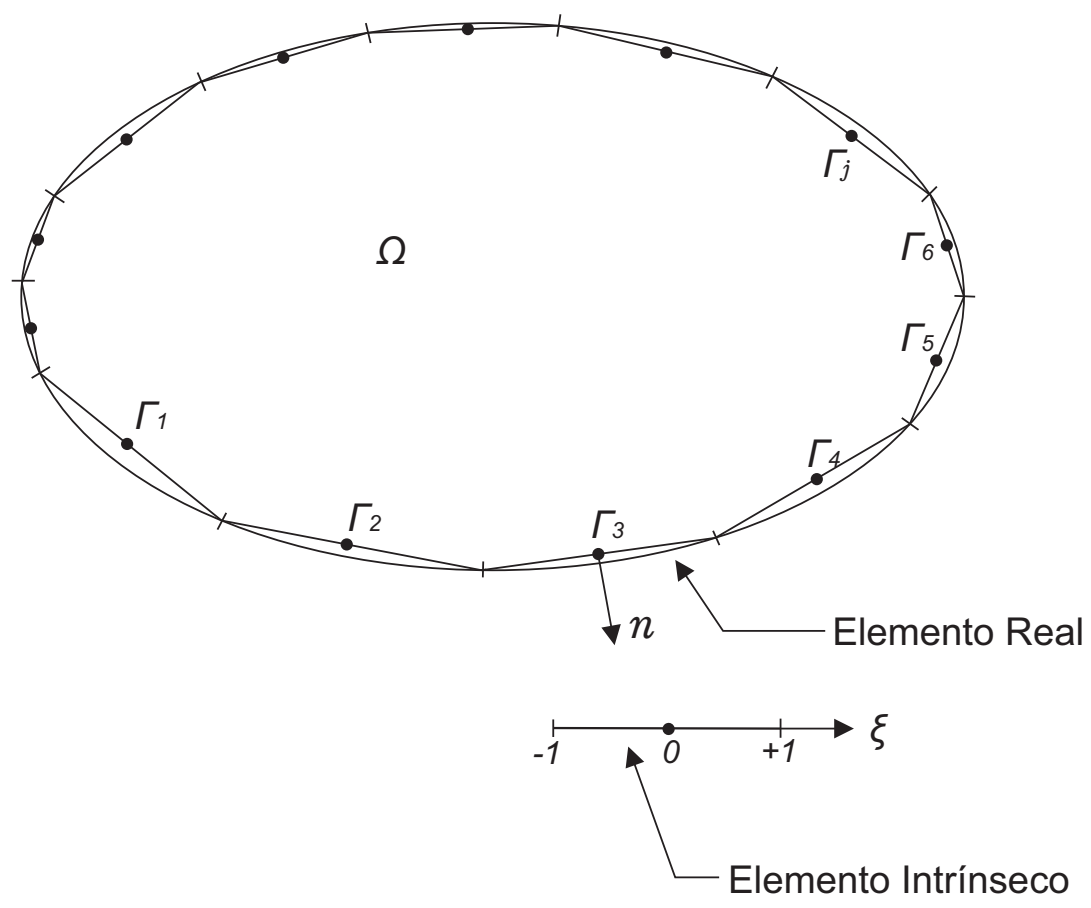

Figura 5.1: Domínio bidimensional dividido em elementos de contorno.

A cada elemento de contorno, associa-se um elemento chamado nó ou pontos nodais e os valores das variáveis associadas a estes são denominados valores nodais, ver Figura 5.2. Considerando $u_{j}$ e $t_{j}$ constantes ao longo do elemento $j$.

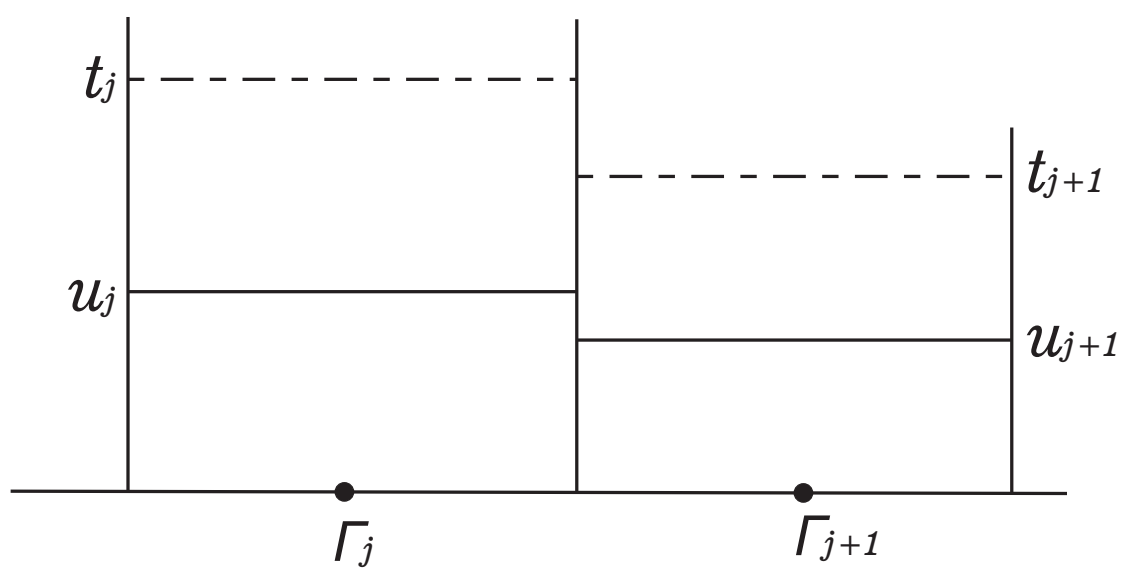

Figura 5.2: Deslocamento e tração no contorno. 
Após a discretização, a equação (5.32) pode ser reescrita como:

$$
\begin{aligned}
\frac{1}{2} u_{i}\left(x^{\prime}\right) & +\sum_{j=1}^{N_{e}} u_{j} \int_{\xi=-1}^{x=+1} P_{i j}^{*}\left(x^{\prime}, x(\xi)\right) J d \xi=\sum_{j=1}^{N_{e}} p_{j} \int_{\xi=-1}^{x=+1} U_{i j}^{*}\left(x^{\prime}, x(\xi)\right) J d \xi \\
& +\sum_{j=1}^{N_{e}} \int_{\xi=-1}^{\xi=+1}\left[V_{i, \alpha}^{*}\left(x^{\prime}, x(\xi)\right)-\frac{\nu}{(1-\nu) \lambda^{2}} U_{i \alpha}^{*}\left(x^{\prime}, x(\xi)\right)\right] q_{i} n_{\alpha}(\xi) J d \xi
\end{aligned}
$$

onde, $J$ é o Jacobiano da transformação (i.e. $d \Gamma=J(\xi) d \xi$ ). Para um elemento constante, $J=L_{e} / 2$, em que $L_{e}$ é o comprimento do elemento e $\xi$ é a coordenada local que varia de $\xi=-1$ a $\xi=+1$, ver Figura 5.1. A equação (5.34) pode ser escrita na seguinte forma:

$$
[H]_{3 N_{n} \times 3 N_{n}}\{u\}_{3 N_{n}}=[G]_{3 N_{n} \times 3 N_{n}}\{p\}_{3 N_{n}}+\{Q\}_{3 N_{n} \times 1}
$$

onde $[H]$ e $[G]$ são as matrizes influência, $[Q]$ é o vetor carga no domínio e $N_{n}$ é o número de nós do contorno (para elemento constante: $N_{n}=N_{e}$ ).

\subsection{Tratamento de singularidades}

A matrix de influência $[G]$ e o vetor carga $\{Q\}$ contém integrais com singularidade fraca, que podem ser canceladas usando uma transformação de coordenadas não-linear (ver Apêndice E). A matrix de influência $[H]$ contém integrais fortemente singulares, que podem ser avaliados usando movimento de corpo rígido generalizado. Se um problema de tração-livre é considerado, três casos independentes podem ser observados (Rashed, 1999):

1. $u_{1}=1, u_{2}=0$ e $u_{3}=-r_{1}$ (rotação de corpo rígido em torno de $x_{1}$ )

2. $u_{2}=1, u_{1}=0$ e $u_{3}=-r_{2}$ (rotação de corpo rígido em torno de $x_{2}$ )

3. $u_{3}=1, u_{1}=0$ e $u_{2}=0$ (translação de corpo rígido em torno de $x_{3}$ ) 
Por aplicação dos casos acima ao sistema de equações em (5.37), as seguintes expressões podem ser escritas:

$$
\begin{gathered}
H_{i \alpha}\left(x^{\prime}\right)=-\int_{\Gamma}\left[P_{i \alpha}^{*}\left(x^{\prime}, x\right)+\left(-r_{\alpha}\right) P_{i 3}^{*}\left(x^{\prime}, x\right)\right] d \Gamma(x) \\
H_{i 3}\left(x^{\prime}\right)=-\int_{\Gamma} P_{i 3}^{*}\left(x^{\prime}, x\right) \Gamma(x)
\end{gathered}
$$

onde $H_{i \alpha}\left(x^{\prime}\right)$ e $H_{i 3}\left(x^{\prime}\right)$ incluem a diagonal da sub-matriz e o termo na influência da matriz $[H]$. O primeiro termo na equação (5.38) e (5.39) já foram calculados, entretando, o segundo termo na equação (5.38) tem de ser calculado. Neste termo, a distância $r_{\alpha}$ cancela a singularidade fraca no kernel $P_{\alpha 3}^{*}$ e a singularidade forte no kernel $P_{33}^{*}$.

\subsection{Sistema de equações}

Impondo as condições de contorno (ver seção 4.7), a equação (5.37) pode ser escrita da seguinte forma:

$$
[A]_{3 N_{n} \times 3 N_{n}}\{x\}_{3 N_{n} \times 1}=\{b\}_{3 N_{n} \times 1}
$$

onde $[A]$ é a matriz do sistema, $\{x\}$ é o vetor das variáveis desconhecidas e $\{b\}$ é o vetor com as condições de contorno conhecidas.

\subsection{Tensões em pontos internos}

Uma vez que os valores para o contorno são calculados, os deslocamentos em pontos internos $X^{\prime}$ podem ser avaliados usando a equação (5.32) com $c_{i j}=\delta_{i j}$. Tensões de flexão e cisalhamento em pontos internos são obtidas diferenciando a equação (5.32) em relação a coordenada do ponto fonte $X^{\prime}$ e substituindo então as equações (4.66) e (4.67): 


$$
\begin{array}{r}
M_{\alpha \beta}\left(X^{\prime}\right)=\int_{\Gamma} U_{\alpha \beta \gamma}^{*}\left(X^{\prime}, x\right) p_{k}(x) d \Gamma(x)-\int_{\Gamma} P_{\alpha \beta \gamma}^{*}\left(X^{\prime}, x\right) u_{k}(x) d \Gamma(x) \\
+q \int_{\Gamma} W_{\alpha \beta}^{*}\left(X^{\prime}, x\right) d \Gamma(x) \\
Q_{\beta}\left(X^{\prime}\right)=\int_{\Gamma} U_{3 \beta \gamma}^{*}\left(X^{\prime}, x\right) p_{k}(x) d \Gamma(x)-\int_{\Gamma} P_{3 \beta \gamma}^{*}\left(X^{\prime}, x\right) u_{k}(x) d \Gamma(x) \\
+q \int_{\Gamma} W_{3 \beta}^{*}\left(X^{\prime}, x\right) d \Gamma(x)
\end{array}
$$

onde os kernels $U_{i j k}^{*}, P_{i j k}^{*}$ e $W_{i \beta}$ (Vander Weeën, 1982) são dados por:

$$
\begin{gathered}
U_{\alpha \beta \gamma}^{*}=\frac{1}{4 \pi r}\left[\left(4 A(z)+2 z K_{1}(z)+1-\nu\right)\left(\delta_{\beta \gamma} r_{, \alpha}+\delta_{\alpha \gamma} r_{, \beta}\right)\right. \\
\left.-2\left(8 A(z)+2 z K_{1}(z)+1-\nu\right) r_{, \alpha} r_{, \beta} r_{, \gamma}+(4 A(z)+1+\nu) \delta_{\alpha \beta} r_{, \gamma}\right] \\
U_{\alpha \beta 3}^{*}=\frac{-(1-\nu)}{8 \pi}\left[\left(2 \frac{(1+\nu)}{(1-\nu)} \log (z)-1\right) \delta_{\alpha \beta}+2 r_{, \alpha r_{, \beta}}\right] \\
U_{3 \beta \gamma}^{*}=\frac{\lambda^{2}}{2 \pi}\left[B(z) \delta_{\gamma \beta}-A(z) r_{, \gamma} r_{, \beta}\right] \\
U_{3 \beta 3}^{*}=\frac{1}{2 \pi r} r_{, \beta}
\end{gathered}
$$

e

$$
\begin{array}{r}
P_{\alpha \beta \gamma}^{*}=\frac{D(1-\nu)}{4 \pi r^{2}}\left\{\left(4 A(z)+2 z K_{1}(z)+1-\nu\right)\left(\delta_{\gamma \alpha} n_{\beta}+\delta_{\gamma \beta} n_{\alpha}\right)\right. \\
+(4 A(z)+1+3 \nu) \delta_{\alpha \beta} n_{\gamma}-\left(16 A(z)+6 z K_{1}(z)+z^{2} K_{o}(z)+2-2 \nu\right) \\
\times\left[\left(n_{\alpha} r_{, \beta}+n_{\beta} r_{, \alpha}\right) r_{, \gamma}+\left(\delta_{\gamma \alpha} r_{, \beta}+\delta_{\gamma \beta} r_{, \alpha}\right) r_{, n}\right] \\
-2\left(8 A(z)+2 z K_{1}(z)+1+\nu\right)\left(\delta_{\alpha \beta} r_{, \gamma} r_{, n}+n_{\gamma} r_{, \alpha} r_{\beta}\right) \\
\left.+4\left(24 A(z)+8 z K_{1}(z)+z^{2} K_{o}(z)+2-2 \nu\right) r_{, \alpha} r_{, \beta} r_{, \gamma} r_{, n}\right\}
\end{array}
$$




$$
\begin{aligned}
& P_{\alpha \beta 3}^{*}=\frac{D(1-n u) \lambda^{2}}{4 \pi r}\left[\left(2 A(z)+z K_{1}(z)\right)\left(r_{, \beta} n_{\alpha}+r_{, \alpha} n_{\beta}\right)\right. \\
& \left.-2\left(4 A(z)+z K_{1}(z)\right) r_{, \alpha} r_{, \beta} r_{, n}+2 A(z) \delta_{\alpha \beta} r_{, n}\right] \\
& P_{3 \beta 3}^{*}=-\frac{D(1-n u) \lambda^{2}}{4 \pi r}\left[\left(2 A(z)+z K_{1}(z)\right)\left(\delta_{\gamma \beta} r_{n}+r_{, \gamma} n_{\beta}\right)\right. \\
& \left.+2 A(z) n_{\gamma} r_{, \beta}-2\left(4 A(z)+z K_{1}(z)\right) r_{, \alpha} r_{, \beta} r_{, n}\right] \\
& P_{3 \beta 3}^{*}=\frac{D(1-n u) \lambda^{2}}{4 \pi r^{2}}\left[\left(z^{2} B(z)+1\right) n_{\beta}-\left(z^{2} A(z)+2\right) r_{, \beta} r_{, n}\right] \\
& W_{\alpha \beta}^{*}=\frac{-r}{64 \pi}\left\{(4 \log (z)-3)\left[(1+\nu)\left(r_{, \beta} n_{\alpha}+r_{, \alpha} n_{\beta}\right)+(1+3 \nu) \delta_{\alpha \beta} r_{, n}\right]\right. \\
& \left.4\left[(1-\nu) r_{, \alpha} r_{, \beta}+\nu \delta_{\alpha \beta}\right] r_{, n}\right\}-\frac{\nu}{(1-\nu) \lambda^{2}} U_{\alpha \beta \gamma}^{*} n_{\gamma} \\
& W_{3 \beta}^{*}=\frac{1}{8 \pi}\left[(2 \log (z)-1) n_{\beta}+2 r_{, \beta} r_{, n}\right]-\frac{\nu}{(1-\nu) \lambda^{2}} U_{3 \beta \gamma}^{*} n_{\gamma}
\end{aligned}
$$

Consequentemente, a tensão normal e a de cisalhamento através da espessura podem ser avaliadas usando as equações (4.60), (4.61) e (4.62).

\subsection{Equação integral hipersingular}

A equação (5.32) é também válida para um ponto externo. Dessa forma, a equação (5.41) e (5.42) é escrita com $c_{i j}=0$, ou seja, $X " \notin \Omega$ :

$$
\int_{\Gamma} P_{\alpha \beta k}^{*}\left(X^{\prime \prime}, x\right) u_{k}(x) d \Gamma(x)=\int_{\Gamma} U_{\alpha \beta k}^{*}\left(X^{\prime \prime}, x\right) p_{k}(x) d \Gamma(x)+q \int_{\Gamma} W_{\alpha \beta}^{*}\left(X^{\prime \prime}, x\right) d \Gamma(x)
$$




$$
\int_{\Gamma} P_{3 \beta k}^{*}\left(X^{\prime \prime}, x\right) u_{k}(x) d \Gamma(x)=\int_{\Gamma} U_{3 \beta k}^{*}\left(X^{\prime \prime}, x\right) p_{k}(x) d \Gamma(x)+q \int_{\Gamma} W_{3 \beta}^{*}\left(X^{\prime \prime}, x\right) d \Gamma(x)
$$

onde os kernels $U_{i j k}^{*}, P_{i j k}^{*}$ e $W_{i j k}^{*}$ são dados na seção 5.7. As tensões resultantes da equação integral de contorno são formadas por considerações no comportamento das equações (5.53) e (5.54) quando o ponto $X$ " tende ao contorno $\Gamma$ em $x^{\prime}$. Para satisfazer a continuidade, o ponto $x^{\prime}$ é assumido sobre um contorno suave. Um domínio semicircular com contorno $\Gamma_{\varepsilon}^{*}$ é construído ao redor do ponto $x^{\prime}$ (ver Figura 5.3).

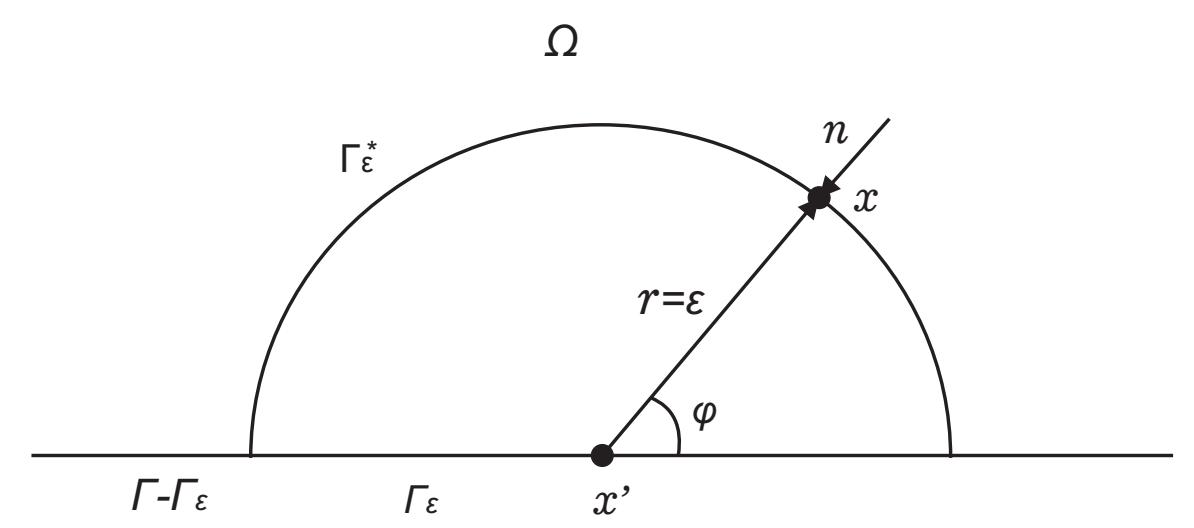

Figura 5.3: Região semicircular em torno do ponto fonte.

Fazendo o limite como $X^{\prime \prime} \rightarrow x^{\prime}$, as equações (5.53) e (5.54) podem ser reescritas da seguinte forma:

$$
\begin{array}{r}
\lim _{\varepsilon \rightarrow 0} \int_{\Gamma-\Gamma_{\varepsilon}+\Gamma_{\varepsilon}^{*}} P_{\alpha \beta \gamma}^{*}\left(x^{\prime}, x\right) u_{\gamma}(x) d \Gamma(x)+\lim _{\varepsilon \rightarrow 0} \int_{\Gamma-\Gamma_{\varepsilon}+\Gamma_{\varepsilon}^{*}} P_{\alpha \beta 3}^{*}\left(x^{\prime}, x\right) u_{3}(x) d \Gamma(x) \\
=\lim _{\varepsilon \rightarrow 0} \int_{\Gamma-\Gamma_{\varepsilon}+\Gamma_{\varepsilon}^{*}} U_{\alpha \beta \gamma}^{*}\left(x^{\prime}, x\right) p_{\gamma}(x) d \Gamma(x)+\lim _{\varepsilon \rightarrow 0} \int_{\Gamma-\Gamma_{\varepsilon}+\Gamma_{\varepsilon}^{*}} U_{\alpha \beta 3}^{*}\left(x^{\prime}, x\right) p_{3}(x) d \Gamma(x) \\
+q \lim _{\varepsilon \rightarrow 0} \int_{\Gamma-\Gamma_{\varepsilon}+\Gamma_{\varepsilon}^{*}} W_{\alpha \beta}^{*}\left(x^{\prime}, x\right) d \Gamma(x)
\end{array}
$$

e

$$
\lim _{\varepsilon \rightarrow 0} \int_{\Gamma-\Gamma_{\varepsilon}+\Gamma_{\varepsilon}^{*}} P_{3 \beta \gamma}^{*}\left(x^{\prime}, x\right) u_{\gamma}(x) d \Gamma(x)+\lim _{\varepsilon \rightarrow 0} \int_{\Gamma-\Gamma_{\varepsilon}+\Gamma_{\varepsilon}^{*}} P_{3 \beta 3}^{*}\left(x^{\prime}, x\right) u_{3}(x) d \Gamma(x)
$$




$$
\begin{array}{r}
=\lim _{\varepsilon \rightarrow 0} \int_{\Gamma-\Gamma_{\varepsilon}+\Gamma_{\varepsilon}^{*}} U_{3 \beta \gamma}^{*}\left(x^{\prime}, x\right) p_{\gamma}(x) d \Gamma(x)+\lim _{\varepsilon \rightarrow 0} \int_{\Gamma-\Gamma_{\varepsilon}+\Gamma_{\varepsilon}^{*}} U_{3 \beta 3}^{*}\left(x^{\prime}, x\right) p_{3}(x) d \Gamma(x) \\
+q \lim _{\varepsilon \rightarrow 0} \int_{\Gamma-\Gamma_{\varepsilon}+\Gamma_{\varepsilon}^{*}} W_{3 \beta}^{*}\left(x^{\prime}, x\right) d \Gamma(x)
\end{array}
$$

As equações (5.55) e (5.56) representam a integral de contorno dos esforços resultantes (momento fletor e força cortante), respectivamente, no ponto $x^{\prime}$ do contorno.

\subsubsection{Equação integral de flexão}

A equação (5.55) pode ser escrita da seguinte forma:

$$
I_{1}^{*}+I_{2}^{*}=I_{3}^{*}+I_{4}^{*}+I_{5}^{*}
$$

Os valores no contorno de $u_{i}$ são assumidos como $C^{1, \alpha},(0<\alpha<1)$ para permitir a expansão na série de Taylor até dois termos. Em seguida, cada uma das integrais da equação (5.57) será expandida e considerada individualmente.

\subsubsection{Integral $I_{1}^{*}$}

A integral $I_{1}$ pode ser escrita da seguinte forma:

$$
\begin{array}{r}
I_{1}^{*}=\lim _{\varepsilon \rightarrow 0} \int_{\Gamma-\Gamma_{\varepsilon}+\Gamma_{\varepsilon}^{*}} P_{\alpha \beta \gamma}^{*}\left(x^{\prime}, x\right) u_{\gamma}(x) d \Gamma(x)=\lim _{\varepsilon \rightarrow 0} \int_{\Gamma-\Gamma_{\varepsilon}} P_{\alpha \beta \gamma}^{*}\left(x^{\prime}, x\right) u_{\gamma}(x) d \Gamma(x) \\
+\lim _{\varepsilon \rightarrow 0} \int_{\Gamma_{\varepsilon}^{*}} P_{\alpha \beta \gamma}^{*}\left(x^{\prime}, x\right)\left[u_{\gamma}(x)-u_{\gamma}\left(x^{\prime}\right)-u_{\gamma, \theta}\left(x^{\prime}\right)\left(x_{\theta}(x)-x_{\theta}\left(x^{\prime}\right)\right)\right] d \Gamma(x) \\
+u_{\gamma}\left(x^{\prime}\right) \lim _{\varepsilon \rightarrow 0} \int_{\Gamma_{\varepsilon}^{*}} P_{\alpha \beta \gamma}^{*}\left(x^{\prime}, x\right) u_{\gamma}(x) d \Gamma(x)+u_{\gamma, \theta}\left(x^{\prime}\right) \lim _{\varepsilon \rightarrow 0} \int_{\Gamma_{\varepsilon}^{*}} P_{\alpha \beta \gamma}^{*}\left(x^{\prime}, x\right)\left(x_{\theta}(x)-x_{\theta}\left(x^{\prime}\right)\right) d \Gamma(x)
\end{array}
$$

Nota-se que a integral $I_{1}^{*}$ contém o kernel $P_{\alpha \beta \gamma}^{*}$ e apresenta singularidade da ordem $1 / r^{2}$. Para este caso, a expansão em apenas dois termos da série de Taylor são apropriados. Na integral acima, o segundo termo do lado direito é zero quando $\varepsilon \rightarrow 0$. O primeiro e o terceiro 
termo do lado direito juntos formam a parte finita de Hadamard. Da Figura 5.3, as seguintes relações podem ser obtidas:

$$
\begin{array}{ll}
r=\varepsilon & r_{, n}=-1 \quad d \Gamma=\varepsilon d \varphi \\
r_{, 1}=-n_{1}=\cos (\varphi) & r_{, 2}=-n_{2}=\operatorname{sen}(\varphi)
\end{array}
$$

e

$$
\int_{T_{\varepsilon}^{*}} d \Gamma=\int_{0}^{\pi} \varepsilon d \varphi
$$

Considerando as relações acima, juntos com os seguintes limites (ver equação (5.28) e $(5.29))$ :

$$
\begin{gathered}
\lim _{\varepsilon \rightarrow 0} A(\lambda \varepsilon)=\frac{-1}{2} \\
\lim _{\varepsilon \rightarrow 0} \lambda^{2} \varepsilon^{2} K_{0}(\lambda \varepsilon)=0 \\
\lim _{\varepsilon \rightarrow 0} \lambda \varepsilon K_{1}(\lambda \varepsilon)=1 \\
\lim _{\varepsilon \rightarrow 0} \lambda^{2} \varepsilon^{2} B(\lambda \varepsilon)=0
\end{gathered}
$$

O último termo no lado direito da equação (5.58) é dado por:

$$
\begin{array}{r}
u_{\gamma, \theta}\left(x^{\prime}\right) \lim _{\varepsilon \rightarrow 0} \int_{\Gamma_{\varepsilon}^{*}} P_{\alpha \beta \gamma}^{*}\left(x^{\prime}, x\right)\left(x_{\theta}(x)-x_{\theta}\left(x^{\prime}\right)\right) d \Gamma(x) \\
=\frac{D(1+\nu)(1-\nu)}{16}\left(u_{\beta, \alpha}\left(x^{\prime}\right)+u_{\alpha, \beta}\left(x^{\prime}\right)+u_{\gamma, \gamma}\left(x^{\prime}\right) \delta_{\alpha \beta}\right)
\end{array}
$$

Agora, a integral $I_{1}^{*}$ pode ser escrita como segue: 


$$
I_{1}^{*}=\int_{\Gamma} P_{\alpha \beta \gamma}^{*}\left(x^{\prime}, x\right) u_{\gamma}(x) d \Gamma(x)+\frac{D(1+\nu)(1-\nu)}{16}\left(u_{\beta, \alpha}\left(x^{\prime}\right)+u_{\alpha, \beta}\left(x^{\prime}\right)+u_{\gamma, \gamma}\left(x^{\prime}\right) \delta_{\alpha \beta}\right)
$$

\subsubsection{Integral $I_{2}^{*}$}

A integral $I_{2}^{*}$ pode ser tratada de forma similar a integral $I_{1}^{*}$. Somente um termo na série de Taylor é necessário para $I_{2}^{*}$, pois o kernel $P_{\alpha \beta 3}$ contém singularidade forte do tipo $1 / r$. Assim, a integral $I_{2}^{*}$ pode ser escrita da seguinte forma:

$$
\begin{aligned}
& I_{2}^{*}=\lim _{\varepsilon \rightarrow 0} \int_{\Gamma-\Gamma_{\varepsilon}+\Gamma_{\varepsilon}^{*}} P_{\alpha \beta 3}^{*}\left(x^{\prime}, x\right) u_{3}(x) d \Gamma(x)=\lim _{\varepsilon \rightarrow 0} \int_{\Gamma-\Gamma_{\varepsilon}} P_{\alpha \beta 3}^{*}\left(x^{\prime}, x\right) u_{3}(x) d \Gamma(x) \\
& +\lim _{\varepsilon \rightarrow 0} \int_{\Gamma_{\varepsilon}^{*}} P_{\alpha \beta 3}^{*}\left(x^{\prime}, x\right)\left[u_{3}(x)-u_{3}\left(x^{\prime}\right)\right] d \Gamma(x)+u_{3}\left(x^{\prime}\right) \lim _{\varepsilon \rightarrow 0} \int_{\Gamma_{\varepsilon}^{*}} P_{\alpha \beta 3}^{*}\left(x^{\prime}, x\right) d \Gamma(x)
\end{aligned}
$$

Na integral acima, o segundo termo do lado direito é zero quando $\varepsilon \rightarrow 0$. O primeiro termo no lado direito forma uma integral no valor principal de Cauchy. Considerando as relações na equação (5.60) e os limites dados através das equações (5.61) à (5.64), o último termo no lado direito desaparece. Assim, a integral $I_{2}^{*}$ pode ser escrita como segue:

$$
I_{2}^{*}=\int_{\Gamma} P_{\alpha \beta 3}\left(x^{\prime}, x\right) u_{3}(x) d \Gamma(x)
$$

\subsubsection{Integral $I_{3}^{*}$}

A integral $I_{3}^{*}$ que contém $U_{\alpha \beta \gamma}$ que é fortemente singular. Usando o primeiro termo da série de Taylor de $M_{\gamma \theta}$, tem-se:

$$
\begin{array}{r}
I_{3}^{*}=\lim _{\varepsilon \rightarrow 0} \int_{\Gamma-\Gamma_{\varepsilon}+\Gamma_{\varepsilon}^{*}} U_{\alpha \beta \gamma}^{*}\left(x^{\prime}, x\right) p_{\gamma}(x) d \Gamma(x)=\lim _{\varepsilon \rightarrow 0} \int_{\Gamma-\Gamma_{\varepsilon}} U_{\alpha \beta \gamma}^{*}\left(x^{\prime}, x\right) p_{\gamma}(x) d \Gamma(x) \\
+\lim _{\varepsilon \rightarrow 0} \int_{\Gamma_{\varepsilon}^{*}} U_{\alpha \beta \gamma}^{*}\left(x^{\prime}, x\right) n_{\theta}(x)\left[M_{\gamma \theta}(x)-M_{\gamma \theta}\left(x^{\prime}\right)\right] d \Gamma(x) \\
+M_{\gamma \theta}\left(x^{\prime}\right) \lim _{\varepsilon \rightarrow 0} \int_{\Gamma_{\varepsilon}^{*}} U_{\alpha \beta \gamma}^{*}\left(x^{\prime}, x\right) n_{\theta}(x) d \Gamma(x)
\end{array}
$$


Na integral acima, o segundo termo no lado direito é zero quando $\varepsilon \rightarrow 0$. O primeiro termo no lado direito forma uma integral no valor principal de Cauchy. Considerando as relações na equação (5.60) e os limites dados através das equações (5.61) à (5.64), o último termo no lado direito é escrito da seguinte forma:

$$
M_{\gamma \theta}\left(x^{\prime}\right) \lim _{\varepsilon \rightarrow 0} \int_{\Gamma_{\varepsilon}^{*}} U_{\alpha \beta \gamma}^{*}\left(x^{\prime}, x\right) n_{\theta}(x) d \Gamma(x)=\left(\frac{(3 \nu-1)}{16} M_{\gamma \gamma}\left(x^{\prime}\right) \delta_{\alpha \beta}-\frac{2(\nu-3)}{16} M_{\alpha \beta}\left(x^{\prime}\right)\right)
$$

Agora, a integral $I_{3}^{*}$ pode ser escrita como segue:

$$
I_{3}^{*}=\int_{\Gamma} U_{\alpha \beta \gamma}^{*}\left(x^{\prime}, x\right) p_{\gamma}(x) d \Gamma(x)-\left(\frac{(3 \nu-1)}{16} M_{\gamma \gamma}\left(x^{\prime}\right) \delta_{\alpha \beta}-\frac{2(\nu-3)}{16} M_{\alpha \beta}\left(x^{\prime}\right)\right)
$$

\subsubsection{Integral $I_{4}^{*}$}

Esta integral contém $U_{\alpha \beta 3}^{*}$ que é um kernel fracamente singular. A integral $I_{4}$ pode ser escrita da seguinte forma:

$$
I_{4}^{*}=\int_{\Gamma} U_{\alpha \beta 3}^{*} p_{3}(x) d \Gamma(x)
$$

\subsubsection{Integral $I_{5}^{*}$}

Esta integral contém $W_{\alpha \beta}^{*}$ que é um kernel fortemente singular. Pode-se escrever $I_{5}^{*}$ da seguinte forma:

$$
\begin{array}{r}
I_{5}^{*}=q \lim _{\varepsilon \rightarrow 0} \int_{\Gamma-\Gamma_{\varepsilon}+\Gamma_{\varepsilon}^{*}} W_{\alpha \beta}^{*}\left(x^{\prime}, x\right) d \Gamma(x) \\
=q \lim _{\varepsilon \rightarrow 0} \int_{\Gamma-\Gamma_{\varepsilon}} W_{\alpha \beta}^{*}\left(x^{\prime}, x\right) d \Gamma(x) \\
+q \lim _{\varepsilon \rightarrow 0} \int_{\Gamma_{\varepsilon}^{*}} W_{\alpha \beta}^{*}\left(x^{\prime}, x\right) d \Gamma(x)
\end{array}
$$


Na integral acima, o primeiro termo do lado direito forma uma integral no valor principal de Cauchy. Considerando as equações (5.60) à (5.64), o último termo do lado direito da equação acima toma a seguinte forma:

$$
q \lim _{\varepsilon \rightarrow 0} \int_{\Gamma_{\varepsilon}^{*}} W_{\alpha \beta}^{*}\left(x^{\prime}, x\right) d \Gamma(x)=\frac{\nu q}{(1-\nu) \lambda_{\alpha \beta}^{2}} \frac{(1+\nu)}{4} \delta_{\alpha \beta}
$$

Agora, a integral $I_{5}^{*}$ pode ser escrita como:

$$
I_{5}^{*}=q \int_{\Gamma} W_{\alpha \beta}^{*}\left(x^{\prime}, x\right) d \Gamma(x)+\frac{\nu q}{(1-\nu) \lambda^{2}} \frac{(1+\nu)}{4} \delta_{\alpha \beta}
$$

Substituindo as equações (5.66), (5.67), (5.71), (5.72) e (5.75) na equação (5.55) e usando as equações (4.66) e (4.67), obtém-se:

$$
\begin{array}{r}
\frac{1}{2} M_{\alpha \beta}\left(x^{\prime}\right)+\int_{\Gamma} P_{\alpha \beta \gamma}^{*}\left(x^{\prime}, x\right) u_{\gamma}(x) d \Gamma(x)+\int_{\Gamma} P_{\alpha \beta 3}^{*}\left(x^{\prime}, x\right) u_{3}(x) d \Gamma(x) \\
=\int_{\Gamma} U_{\alpha \beta \gamma}^{*}\left(x^{\prime}, x\right) p_{\gamma}(x) d \Gamma(x)+\int_{\Gamma} U_{\alpha \beta 3}^{*}\left(x^{\prime}, x\right) p_{3}(x) d \Gamma(x) \\
+q \int_{\Gamma} W_{\alpha \beta}^{*}\left(x^{\prime}, x\right) d \Gamma(x)+\frac{1}{2} \frac{q \nu}{(1-\nu) \lambda^{2}} \delta_{\alpha \beta}
\end{array}
$$

que é a equação integral de contorno de flexão.

\subsubsection{Equação integral da força cortante}

A equação (5.56) pode ser escrita da seguinte forma:

$$
I_{6}^{*}+I_{7}^{*}=I_{8}^{*}+I_{9}^{*}+I_{10}^{*}
$$

Em sequência, cada integral de $I_{6}^{*}$ à $I_{10}^{*}$ será considerada individualmente. 


\subsubsection{Integral $I_{6}^{*}$}

Esta integral contém $P_{3 \alpha \gamma}^{*}$ que é fortemente singular. Usando o primeiro termo da série de Taylor, $u_{\gamma}$ pode ser escrita da seguinte forma:

$$
\begin{array}{r}
I_{6}^{*}=\lim _{\varepsilon \rightarrow 0} \int_{\Gamma-\Gamma_{\varepsilon}+\Gamma_{\varepsilon}^{*}} P_{3 \beta \gamma}^{*}\left(x^{\prime}, x\right) u_{\gamma}(x) d \Gamma(x) \\
=\lim _{\varepsilon \rightarrow 0} \int_{\Gamma-\Gamma_{\varepsilon}} P_{3 \beta \gamma}^{*}\left(x^{\prime}, x\right) u_{\gamma}(x) d \Gamma(x) \\
+\lim _{\varepsilon \rightarrow 0} \int_{\Gamma_{\varepsilon}^{*}} P_{3 \beta \gamma}^{*}\left(x^{\prime}, x\right)\left[u_{\gamma}(x)-u_{\gamma}\left(x^{\prime}\right)\right] d \Gamma(x) \\
+u_{\gamma}\left(x^{\prime}\right) \lim _{\varepsilon \rightarrow 0} P_{3 \beta \gamma}^{*}\left(x^{\prime}, x\right) d \Gamma(x)
\end{array}
$$

$\mathrm{Na}$ integral acima o segundo termo do lado direito é zero no limite $\varepsilon \rightarrow 0$. O primeiro termo forma a integral no valor principal de Cauchy. Considerando as equações (5.60) à (5.64), o último termo pode ser escrito da seguinte forma:

$$
u_{\gamma}\left(x^{\prime}\right) \lim _{\varepsilon \rightarrow 0} \int_{\Gamma_{\varepsilon}^{*}} P_{3 \beta \gamma}^{*}\left(x^{\prime}, x\right) d \Gamma(x)=\frac{D(1-\nu) \lambda^{2}}{8} u_{\beta}\left(x^{\prime}\right)
$$

Então a integral $I_{6}^{*}$ pode ser escrita na seguinte forma:

$$
I_{6}^{*}=\int_{\Gamma} P_{3 \beta \gamma}^{*}\left(x^{\prime}, x\right) u_{\gamma}(x) d \Gamma(x)+\frac{D(1-\nu) \lambda^{2}}{8} u_{\beta}\left(x^{\prime}\right)
$$

\subsubsection{Integral $I_{7}^{*}$}

Esta integral contém o kernel $P_{3 \beta 3}^{*}$ que é um kernel hipersingular. Usando o primeiro termo da série de Taylor para $u_{3}$, o termo $I_{7}^{*}$ pode ser escrito da seguinte forma:

$$
\begin{array}{r}
I_{7}^{*}=\lim _{\varepsilon \rightarrow 0} \int_{\Gamma-\Gamma_{\varepsilon}+\Gamma_{\varepsilon}^{*}} P_{3 \beta 3}^{*}\left(x^{\prime}, x\right) u_{3}(x) d \Gamma(x) \\
=\lim _{\varepsilon \rightarrow 0} \int_{\Gamma-\Gamma_{\varepsilon}} P_{3 \beta 3}^{*}\left(x^{\prime}, x\right) u_{3}(x) d \Gamma(x) \\
+\lim _{\varepsilon \rightarrow 0} \int_{\Gamma_{\varepsilon}^{*}} P_{3 \beta 3}^{*}\left(x^{\prime}, x\right)\left[u_{3}(x)-u_{3}\left(x^{\prime}\right)-u_{3, \alpha}\left(x^{\prime}\right)\left(x_{\alpha}(x)-x_{\alpha}\left(x^{\prime}\right)\right)\right] d \Gamma(x)
\end{array}
$$




$$
\begin{array}{r}
+u_{3}\left(x^{\prime}\right) \lim _{\varepsilon \rightarrow 0} \int_{\Gamma_{\varepsilon}^{*}} P_{3 \beta 3}^{*}\left(x^{\prime}, x\right) d \Gamma(x) \\
+u_{3, \alpha} \lim _{\varepsilon \rightarrow 0} \int_{\Gamma_{\varepsilon}^{*}} P_{3 \beta 3}^{*}\left(x^{\prime}, x\right)\left(x_{\alpha}(x)-x_{\alpha}\left(x^{\prime}\right)\right) d \Gamma(x)
\end{array}
$$

$\mathrm{Na}$ integral acima, o segundo termo no lado direito é zero quando $\varepsilon \rightarrow 0$. O último e terceiro termo juntos formam a parte finita da Hadamard. Considerando as equações (5.60) à (5.64), o último termo de $I_{7}^{*}$ pode ser escrito da seguinte forma:

$$
\begin{array}{r}
u_{3, \alpha}\left(x^{\prime}\right)=\lim _{\varepsilon \rightarrow 0} \int_{\Gamma_{\varepsilon}^{*}} P_{3 \beta 3}^{*}\left(x^{\prime}, x\right)\left(x_{\alpha}(x)-x_{\alpha}\left(x^{\prime}\right)\right) d \Gamma(x) \\
-\frac{D(1-\nu) \lambda^{2}}{8} u_{3, \beta}\left(x^{\prime}\right)
\end{array}
$$

Agora, a integral $I_{7}^{*}$ pode ser escrita como:

$$
I_{7}^{*}=\int_{\Gamma} P_{3 \beta 3}^{*}\left(x^{\prime}, x\right) u_{3}(x) d \Gamma(x)+\frac{D(1-\nu) \lambda^{2}}{8} u_{3, \beta}\left(x^{\prime}\right)
$$

\subsubsection{Integral $I_{8}^{*}$}

Similar a integral $I_{4}^{*}$, a integral $I_{8}^{*}$ contém o kernel $U_{3 \beta \gamma}^{*}$ que é fracamente singular. A integral $I_{8}^{*}$ pode ser escrita da seguinte forma:

$$
I_{8}^{*}=\int_{\Gamma} U_{3 \beta \gamma}^{*}\left(x^{\prime}, x\right) p_{\gamma}(x) d \Gamma(x)
$$

\subsubsection{Integral $I_{9}^{*}$}

Esta integral contém o kernel $U_{3 \beta \gamma}$ é fortemente singular. Usando o primeiro termo da série de Taylor para $Q_{\theta}$ :

$$
I_{9}^{*}=\lim _{\varepsilon \rightarrow 0} \int_{\Gamma-\Gamma_{\varepsilon}+\Gamma_{\varepsilon}^{*}} U_{3 \beta 3}^{*}\left(x^{\prime}, x\right) p_{3}(x) d \Gamma(x)
$$




$$
\begin{array}{r}
=\lim _{\varepsilon \rightarrow 0} \int_{\Gamma-\Gamma_{\varepsilon}} U_{3 \beta 3}^{*} p_{3}(x) d \Gamma(x) \\
+\lim _{\varepsilon \rightarrow 0} \int_{\Gamma_{\varepsilon}^{*}} U_{3 \beta 3}^{*}\left(x^{\prime}, x\right) n_{\theta}(x)\left[Q_{\theta}(x)-Q_{\theta}\left(x^{\prime}\right)\right] d \Gamma(x) \\
+Q_{\theta}\left(x^{\prime}\right) \lim _{\varepsilon \rightarrow 0} \int_{\Gamma_{\varepsilon}^{*}} U_{3 \beta 3}^{*}\left(x^{\prime}, x\right) n_{\theta}(x) d \Gamma(x)
\end{array}
$$

$\mathrm{Na}$ integral acima, o segundo termo do lado direito é zero quando $\varepsilon \rightarrow 0$. O primeiro termo forma o valor principal de Cauchy. Considerando as equações (5.60) à (5.64), o último termo pode ser escrito da seguinte forma:

$$
Q_{\theta}\left(x^{\prime}\right) \lim _{\varepsilon \rightarrow 0} \int_{\Gamma_{\varepsilon}^{*}} U_{3 \beta 3}^{*}\left(x^{\prime}, x\right) n_{\theta}(x) d \Gamma(x)=-\frac{Q_{\beta}\left(x^{\prime}\right)}{4}
$$

Então a integral $I_{9}^{*}$ pode ser escrita como:

$$
I_{9}^{*}=\int_{\Gamma} U_{3 \beta 3}^{*}\left(x^{\prime}, x\right) p_{3}(x) d \Gamma(x)-\frac{Q_{\beta}\left(x^{\prime}\right)}{4}
$$

\subsubsection{Integral $I_{10}^{*}$}

Esta integral contém o kernel $W_{3 \beta}^{*}$ que é fracamente singular. Então a integral $I_{10}^{*}$ pode ser escrita como:

$$
I_{10}^{*}=q \int_{\Gamma} W_{3 \beta}^{*}\left(x^{\prime}, x\right) d \Gamma(x)
$$

Subsitutindo as equações (5.80), (5.83), (5.84), (5.87) e (5.88) na equação (5.56) e usando as equações (4.66) e (4.67), obtém-se:

$$
\begin{array}{r}
\frac{1}{2} Q_{\beta}\left(x^{\prime}\right)+\int_{\Gamma} P_{3 \beta \gamma}^{*}\left(x^{\prime}, x\right) u_{\gamma}(x) d \Gamma(x)+\int_{\Gamma} P_{3 \beta 3}^{*}\left(x^{\prime}, x\right) u_{3}(x) d \Gamma(x) \\
=\int_{\Gamma} U_{3 \beta \gamma}^{*}\left(x^{\prime}, x\right) p_{\gamma}(x) d \Gamma(x)+\int_{\Gamma} U_{3 \beta 3}^{*}\left(x^{\prime}, x\right) p_{3}(x) d \Gamma(x) \\
+q \int_{\Gamma} W_{3 \beta}^{*}\left(x^{\prime}, x\right) d \Gamma(x)
\end{array}
$$




\subsection{Tensões no contorno}

As tensões no contorno podem ser calculadas usando-se a derivada dos deslocamentos e então o uso da lei de Hooke generalizada (equações (4.66) e (4.67)) ou então pelo uso das equações integrais:

$$
\begin{array}{r}
\frac{1}{2} M_{\alpha \beta}\left(x^{\prime}\right)+\int_{\Gamma} P_{\alpha \beta \gamma}^{*}\left(x^{\prime}, x\right) u_{\gamma}(x) d \Gamma(x)+\int_{\Gamma} P_{\alpha \beta 3}^{*}\left(x^{\prime}, x\right) u_{3}(x) d \Gamma(x) \\
=\int_{\Gamma} U_{\alpha \beta \gamma}^{*}\left(x^{\prime}, x\right) p_{\gamma}(x) d \Gamma(x)+\int_{\Gamma} U_{\alpha \beta 3}^{*}\left(x^{\prime}, x\right) p_{3}(x) d \Gamma(x) \\
+q \int_{\Gamma} W_{\alpha \beta}^{*}\left(x^{\prime}, x\right) d \Gamma(x)+\frac{1}{2} \frac{q \nu}{(1-\nu) \lambda^{2}} \delta_{\alpha \beta}
\end{array}
$$

que é a equação integral de contorno de flexão. A equação para o cálculo do esforço cisalhante é dada por:

$$
\begin{array}{r}
\frac{1}{2} Q_{\beta}\left(x^{\prime}\right)+\int_{\Gamma} P_{3 \beta \gamma}^{*}\left(x^{\prime}, x\right) u_{\gamma}(x) d \Gamma(x)+\int_{\Gamma} P_{3 \beta 3}^{*}\left(x^{\prime}, x\right) u_{3}(x) d \Gamma(x) \\
=\int_{\Gamma} U_{3 \beta \gamma}^{*}\left(x^{\prime}, x\right) p_{\gamma}(x) d \Gamma(x)+\int_{\Gamma} U_{3 \beta 3}^{*}\left(x^{\prime}, x\right) p_{3}(x) d \Gamma(x) \\
+q \int_{\Gamma} W_{3 \beta}^{*}\left(x^{\prime}, x\right) d \Gamma(x)
\end{array}
$$

As equações (5.90) e (5.91) representam a integral resultante de tensões num ponto qualquer $x^{\prime}$ do contorno $\Gamma$ (ver Figura 4.1).

\subsection{Equação integral de tração}

As equações (5.90) e (5.91) podem ser relacionadas com as equações (4.21) e (4.22), levando-se em conta o sentido da normal $\left(n_{\beta}\right)$. Para um ponto de colocação $x^{\prime}$, as duas equações integrais podem ser reescritas da seguinte forma: 


$$
\begin{array}{r}
\frac{1}{2} p_{\alpha}\left(x^{\prime}\right)+n_{\beta}\left(x^{\prime}\right) \int_{\Gamma} P_{\alpha \beta \gamma}^{*}\left(x^{\prime}, x\right) u_{\gamma}(x) d \Gamma(x)+n_{\beta}\left(x^{\prime}\right) \int_{\Gamma} P_{\alpha \beta 3}^{*}\left(x^{\prime}, x\right) u_{3}(x) d \Gamma(x) \\
=n_{\beta}\left(x^{\prime}\right) \int_{\Gamma} U_{\alpha \beta \gamma}^{*}\left(x^{\prime}, x\right) p_{\gamma}(x) d \Gamma(x)+n_{\beta}\left(x^{\prime}\right) \int_{\Gamma} U_{\alpha \beta 3}^{*}\left(x^{\prime}, x\right) p_{3}(x) d \Gamma(x) \\
+q n_{\beta}\left(x^{\prime}\right) \int_{\Gamma} W_{\alpha \beta}^{*}\left(x^{\prime}, x\right) d \Gamma(x)+\frac{1}{2} \frac{q \nu}{(1-\nu) \lambda^{2}} \delta_{\alpha \beta} n_{\beta}\left(x^{\prime}\right)
\end{array}
$$

e

$$
\begin{array}{r}
\frac{1}{2} p_{3}\left(x^{\prime}\right)+n_{\beta}\left(x^{\prime}\right) \int_{\Gamma} P_{3 \beta \gamma}^{*}\left(x^{\prime}, x\right) u_{\gamma}(x) d \Gamma(x)+n_{\beta}\left(x^{\prime}\right) \int_{\Gamma} P_{3 \beta 3}^{*}\left(x^{\prime}, x\right) u_{3}(x) d \Gamma(x) \\
=n_{\beta}\left(x^{\prime}\right) \int_{\Gamma} U_{3 \beta \gamma}^{*}\left(x^{\prime}, x\right) p_{\gamma}(x) d \Gamma(x)+n_{\beta}\left(x^{\prime}\right) \int_{\Gamma} U_{3 \beta 3}^{*}\left(x^{\prime}, x\right) p_{3}(x) d \Gamma(x) \\
+q n_{\beta}\left(x^{\prime}\right) \int_{\Gamma} W_{3 \beta}^{*}\left(x^{\prime}, x\right) d \Gamma(x)
\end{array}
$$

As equações (5.92) e (5.93) representam três equações integrais em termos dos esforços no contorno e podem substituir a equação (5.32). 


\section{Capítulo 6}

\section{Transformação das integrais de domínio em integrais de contorno}

A aplicação do método dos elementos de contorno requer, preferencialmente, que a solução fundamental para o problema em consideração seja conhecida. Essa solução fundamental deve levar em conta todos os termos da equação governante de forma a obter uma formulação onde apenas o contorno é discretizado. Quando isso não for possível, tornamse necessárias técnicas especiais para o tratamento desses termos de domínio. No caso da formulação de placas, os termos devido a cargas distribuídas não é levado em conta na obtenção da solução fundamental. Desta forma, um termo de domínio permanece na integral de domínio. Esta integral deve ser transformada em uma integral de contorno para que não seja necessária a discretização do domínio. Um das alternativas é fazer a transformação exata da integral de domínio proveniente da carga distribuída em integral de contorno.

\subsection{Transformação exata}

Como pôde ser visto na equação (5.32), há integrais de domínio na formulação devido a carga distribuída no domínio. Estas integrais podem ser calculadas por integração direta 
na área $\Omega$ (ver Figura 4.1). Contudo, a formulação dos elementos de contorno perde seu principal atrativo que é a discretização somente no contorno. Neste trabalho, as integrais de domínio oriundas das cargas distribuídas são transformadas em integrais de contorno por uma transformação exata (Albuquerque et al., 2006).

Considere a placa da Figura 4.1 sob um carregamento genérico $q$ aplicado em uma área $\Omega$. A equação (5.32) pode ser escrita em coordenadas polares da seguinte forma:

$$
\int_{\Omega} q U_{i 3}^{*} d \Omega=\int_{\Omega} q U_{i 3}^{*} \rho d \rho d \theta
$$

$\mathrm{Ou}$

$$
\int_{\Omega} q U_{i 3}^{*} d \Omega=\int_{\theta} \int_{0}^{r} q w^{*} \rho d \rho d \theta
$$

onde, $r$ é o valor de $\rho$ em um ponto do contorno $\Gamma$.

Definindo $F^{*}$ como a seguinte integral:

$$
F^{*}=\int_{0}^{r} q U_{i 3}^{*} \rho d \rho
$$

pode-se escrever:

$$
\int_{\Omega} q U_{i 3}^{*} d \Omega=\int_{\theta} F^{*} d \theta
$$

Considerando um ângulo infinitesimal $d \theta$ (Figura 6.1), a relação entre o comprimento do arco $r d \theta$ e o comprimento infinitesimal do contorno $d \Gamma$, pode ser escrito como:

$$
\cos \alpha=\frac{r \frac{d \theta}{2}}{\frac{d \Gamma}{2}}
$$

$\mathrm{Ou}$ 


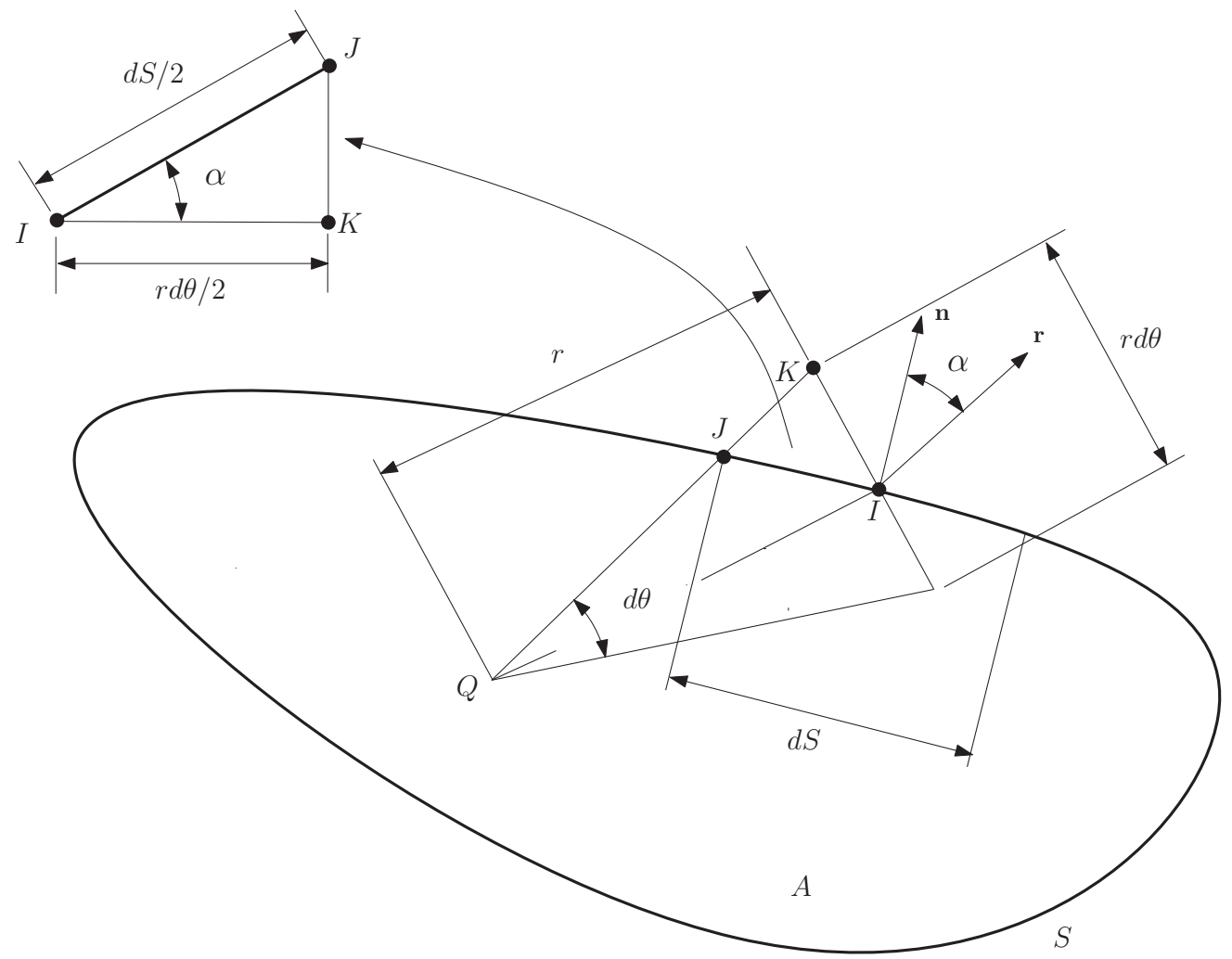

Figura 6.1: Transformação da integral de domínio em integral de contorno.

$$
d \theta=\frac{\cos \alpha}{r} d \Gamma
$$

Usando as propriedades do produto interno dos vetores unitários $\mathbf{n}$ e $\mathbf{r}$, indicados na Figura 6.1, podemos escrever:

$$
d \theta=\frac{\mathbf{n} \cdot \mathbf{r}}{r} d \Gamma
$$

Finalmente, substituindo a equação (6.7) na equação (6.4), a integral de domínio da equação (5.32) pode ser escrita como uma integral de contorno dada por:

$$
\int_{\Omega_{q}} q U_{i 3}^{*} d \Omega=\int_{\Gamma_{g}} \frac{F^{*}}{r} \mathbf{n} \cdot \mathbf{r} d \Gamma
$$

Assim, substituindo a equação (6.8) na equação (5.32), tem-se: 


$$
c_{i j} u_{j}\left(x^{\prime}\right)+f_{\Gamma} P_{i j}^{*}\left(x^{\prime}, x\right) u_{j}(x) d \Gamma(x)=\int_{\Gamma} U_{i j}^{*}\left(x^{\prime}, x\right) p_{j}(x) d \Gamma(x)+\int_{\Gamma_{g}} \frac{F^{*}}{r} \mathbf{n} . \mathbf{r} d \Gamma
$$

Note que agora há somente integrais de contorno na equação (6.9). 


\section{Capítulo 7}

\section{Resultados para placas isotrópicas}

\subsection{Resultados}

Nesta seção, são obtidos resultados numéricos para deslocamentos e esforços (momento e tensão) em placas espessas isotrópicas sujeitas a carregamentos distribuídos em seu domínio. Os valores obtidos para deslocamento, momentos e tensões puderam ser comparados com resultados existentes na literatura (Palermo Jr., 2000, Sanches, 1998 e Bares, 1969). Para tensões os resultados não puderam ser comparados diretamente, visto que, na literatura, não encontrou-se resultados equivalentes ao problema apresentado. Os resultados para tensões foram comparados com o método dos elementos finitos utilizando-se do programa comercial ANSYS. Para todos os problemas seguintes foram estudados a influência do número de elementos no contorno.

\subsubsection{Placa quadrada apoiada nos quatro lados sob carga unifor- memente distribuída}

Considere uma placa apoiada (Figura 7.1) carregada por uma carga $q=1 \mathrm{~N} / \mathrm{m}^{2}$ com sentido contrário a $x_{3}$. A placa é isotrópica e apresenta as seguintes propriedades e dimensões: 
$E=205 \mathrm{GPa}, a=2 \mathrm{~m}$ e espessuras iguais a $h=0,02 \mathrm{~m}(1 \%$ do lado $)$ e $h=0,2 \mathrm{~m}$ (10\% do lado).

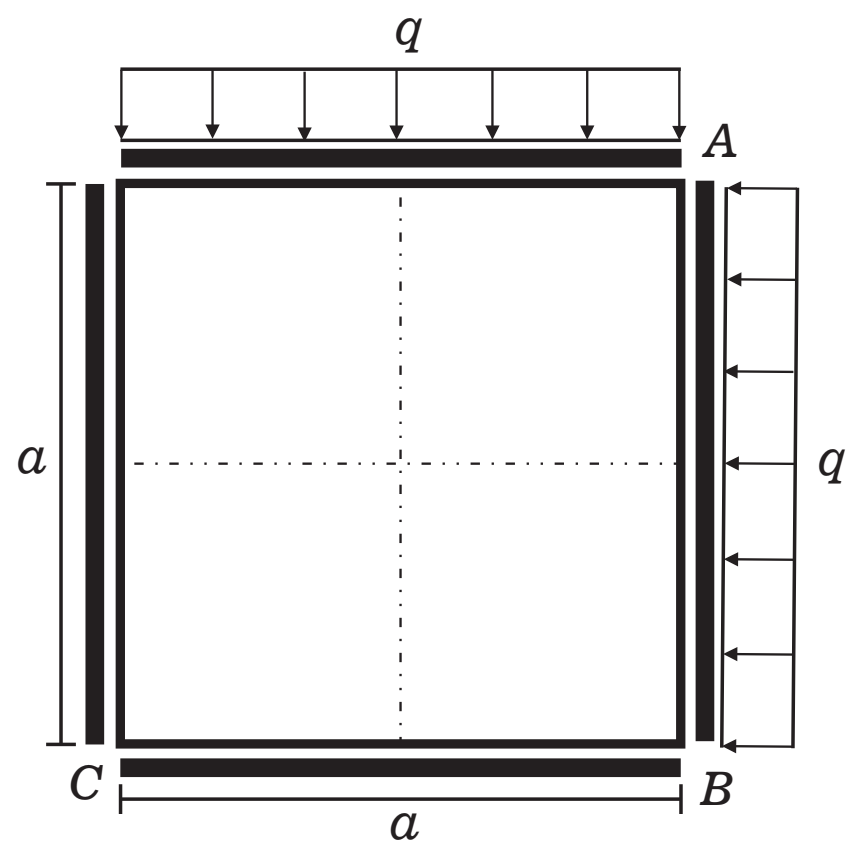

Figura 7.1: Placa quadrada apoiada nos quatro lados.

Para este problema, o contorno foi discretizado usando-se 40 elementos constantes de mesmo comprimento (10 elementos constantes por lado) (ver Figura 7.2). Foram utilizados 4 pontos de integração usando a quadratura de Gauss (Abramowitz e Stegun, 1965) e transformada de Telles (1987) para obter uma boa convergência nos resultados. O principal objetivo da análise é observar o comportamento da espessura em comparação com a solução numérica presente na literatura. Este problema é equivalente ao proposto por Palermo Jr. $(2000)^{1}$. Para placa apoiada as seguintes condições de contorno são conhecidas ao longo dos lados da placa mostrada acima:

$$
u_{t}=0 \longleftrightarrow u_{3}=0 \longleftrightarrow u_{n} \neq 0 \longleftrightarrow M_{t} \neq 0 \longleftrightarrow M_{n}=0
$$

\footnotetext{
${ }^{1}$ Ao longo de todo texto será citado apenas Palermo ao invés de Palermo Jr. afim de minimizar largura das tabelas no texto.
} 


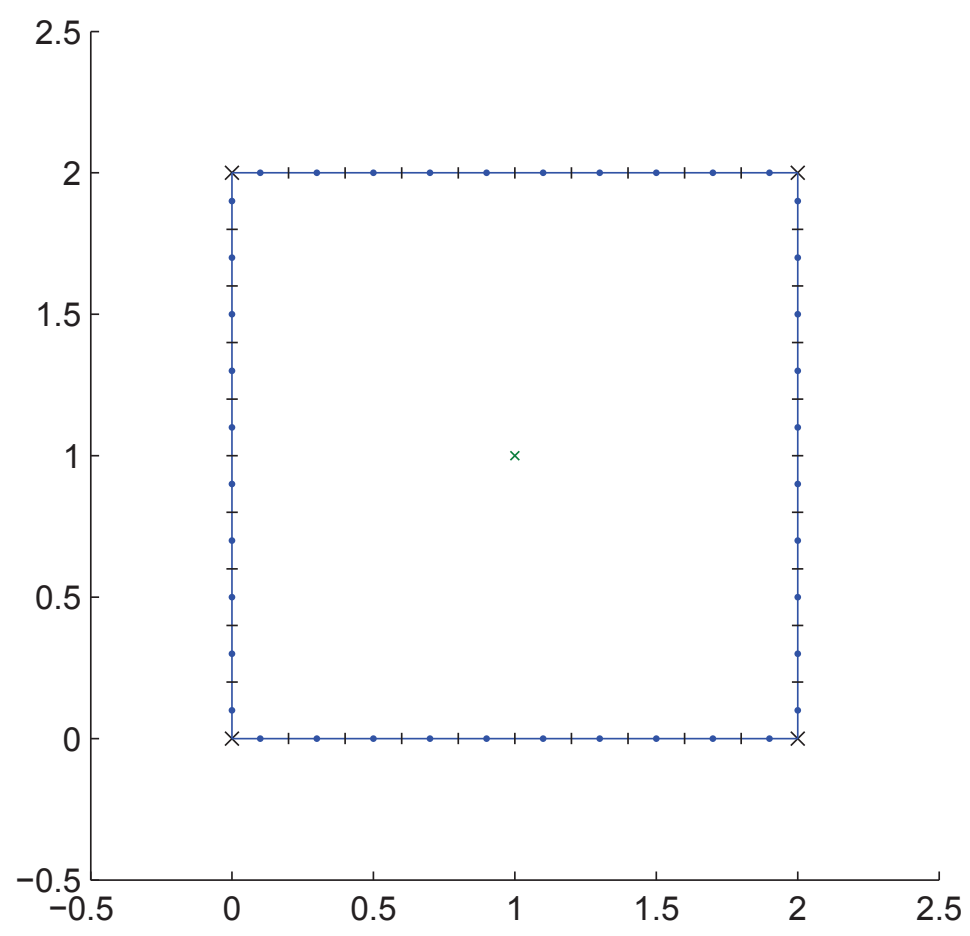

Figura 7.2: Discretização com 40 elementos no contorno e 1 nó interno.

Para se verificar a convergência entre os resultados obtidos com resultados presentes na literatura, foi usada a seguinte fórmula para determinar as diferenças percentuais entre os resultados:

$$
\operatorname{Dif} .(\%)=\left|\frac{s_{\text {ref }}-s_{\text {num }}}{s_{\text {ref }}}\right| \times 100 \%
$$

onde $s_{r e f}$ e $s_{\text {num }}$ são as soluções da referência e numéricas encontradas, respectivamente, para o problema.

a) Coeficiente de Poisson nulo. Ao se considerar o coeficiente de Poisson nulo na equação (5.32) de Reissner, pretende-se observar seus efeitos nos resultados. Dessa forma as integrais de Reissner e Mindlin tornam-se iguais divergindo apenas no fator cisalhante $(\lambda)$ que em Reissner é igual a $\sqrt{10} / h$ e em Mindlin é igual a $\pi / h$. As Tabelas 7.1 e ?? apresentam os resultados para deslocamentos vertical, momentos e tensões no centro da placa. 
Tabela 7.1: Deslocamentos e esforços no centro de uma placa apoiada sujeita a um carregamento distribuído comparados com a condição soft de Palermo (2000).

\begin{tabular}{|c|c|c|c|c|c|c|c|}
\hline \multirow{2}{*}{$h$} & \multicolumn{3}{|c|}{$w \times 10^{-6}(\mathrm{~m})$} & \multicolumn{3}{c|}{$m_{x x}(\mathrm{~N} . \mathrm{m})$} & $\sigma_{x x}\left(\mathrm{~N} / \mathrm{m}^{2}\right)$ \\
\cline { 2 - 8 } & Palermo $(2000)$ & MEC & Dif. $\%$ & Palermo $(2000)$ & MEC & Dif. \% & MEC \\
\hline 0,02 & $-0,487020$ & $-0,488213$ & 0,244 & $-0,149877$ & $-0,150152$ & 0,183 & $-2252,28$ \\
\hline 0,2 & $-554,885$ & $-548,823$ & 1,092 & $-0,161871$ & $-0,160342$ & 0,944 & $-24051,30$ \\
\hline
\end{tabular}

Os resultados obtidos na Tabela 7.1, foram comparados com a solução de Palermo (2000). Os resultados obtidos neste trabalho (MEC) foram obtidos para condição soft (Tabela 7.1), onde liberam-se os momentos volventes no contorno o que implica no aumento de deslocamento e momento. Nota-se que os resultados obtidos para esta condição apresenta diferença nos resultados pouco significativos para placa de espessura $h=0,02 \mathrm{~m}$ e $h=0,2$ m. Segundo, Palermo (2000), na condição soft em que liberam-se os momentos volventes no contorno da placa, este aspecto leva em conta o efeito da cortante, pois é mais adequado para representar os efeitos de borda, ou seja, a liberação dos momentos volventes e dessa forma obtém-se maiores valores para deslocamentos e momentos. Outro fator observado foi a influência do fator cisalhante $(\lambda)$ usado para corrigir o módulo de elasticidade transversal. Notou-se que o $\lambda$ não apresentou qualquer influência nos resultados, apresentando divergências apenas nas últimas casas decimais dos resultados para os resultados de deslocamentos, momentos e tensões, dessa forma, não serão mostrados resultados para este caso. Na Tabela 7.2 foram obtidos resultados para deslocamento vertical, momentos e tensões variando-se a relação espessura/lado. Os resultados obtidos foram comparados com os resultados obtidos pelo método dos elementos finitos usando-se o programa comercial ANSYS. Foi usado o elemento SHELL181 e uma discretização com um total de 10201 pontos nodais. Pode-se notar que os resultados obtidos pelo MEC e MEF apresentam boa concordância para deslocamento vertical, momentos e tensões. As tensões normais $\sigma_{y y}$ apresentaram valores próximos as tensões $\sigma_{x x}$, desta forma, não foram citadas na Tabela 7.2. Os resultados foram obtidos para o centro da placa (ver Figura 7.1). 
Tabela 7.2: Deslocamentos e esforços no centro da placa para diferentes relações espessura/lado.

\begin{tabular}{|c|c|c|c|c|c|c|c|c|c|}
\hline \multirow{2}{*}{$h / a$} & \multicolumn{3}{|c|}{$w \times 10^{-6}(\mathrm{~m})$} & \multicolumn{3}{c|}{$m_{x x}(\mathrm{~N} . \mathrm{m})$} & \multicolumn{3}{c|}{$\sigma_{x x}\left(\mathrm{~N} / \mathrm{m}^{2}\right)$} \\
\cline { 2 - 11 } & MEC & MEF & Dif. $\%$ & MEC & MEF & Dif. $\%$ & MEC & MEF & Dif. $\%$ \\
\hline 0,01 & 0,480 & 0,480 & 0,000 & 0,148 & 0,148 & 0,000 & 2227,3 & 2224,8 & 0,112 \\
\hline 0,02 & 0,060 & 0,060 & 0,000 & 0,149 & 0,149 & 0,000 & 561,15 & 560,85 & 0,053 \\
\hline 0,03 & 0,017 & 0,017 & 0,000 & 0,150 & 0,151 & 0,662 & 251,39 & 252,14 & 0,297 \\
\hline 0,04 & 0,007 & 0,007 & 0,000 & 0,152 & 0,152 & 0,000 & 142,76 & 143,20 & 0,307 \\
\hline
\end{tabular}

\section{b) Coeficiente de Poisson 0,3.}

Na Tabela 7.3 nota-se que o valor do coeficiente de Poisson afetou os deslocamentos, ou seja, houve redução. Por outro lado, aumentou os valores de momento e tensão no nó central da placa. Pode-se notar também que as diferenças nos resultados diminuíram na condição soft como mostrado nas Tabelas 7.1 e 7.3. Na Tabela 7.4 foram obtidos resultados para deslocamentos, momentos e tensões no centro da placa (ponto 1). O aumento no coeficiente de Poisson implicou na diminuição dos deslocamentos e aumento dos momentos e tensões (ver Tabela 7.4), pois houve aumento na rigidez da placa. Os resultados foram obtidos para o centro da placa (ver Figura 7.1).

Tabela 7.3: Deslocamentos e esforços no centro de uma placa apoiada sujeita a um carregamento distribuído comparados com a condição soft de Palermo (2000).

\begin{tabular}{|c|c|c|c|c|c|c|c|}
\hline \multirow{2}{*}{$h$} & \multicolumn{3}{|c|}{$w \times 10^{-6}(\mathrm{~m})$} & \multicolumn{3}{c|}{$m_{x x}(\mathrm{~N} . \mathrm{m})$} & $\sigma_{x x}\left(\mathrm{~N} / \mathrm{m}^{2}\right)$ \\
\cline { 2 - 8 } & Palermo $(2000)$ & MEC & Dif. $\%$ & Palermo $(2000)$ & MEC & Dif. \% & MEC \\
\hline 0,02 & $-0,442154$ & $-0,440081$ & 0,468 & $-0,194545$ & $-0,193838$ & 0,363 & $-2907,57$ \\
\hline 0,2 & $-489,654$ & $-490,421$ & 0,156 & $-204,786$ & $-203,232$ & 0,758 & $-30,48$ \\
\hline
\end{tabular}


Tabela 7.4: Deslocamentos e esforços no centro da placa para diferentes relações espessura/lado.

\begin{tabular}{|c|c|c|c|c|c|c|c|c|c|}
\hline \multirow{2}{*}{$h / a$} & \multicolumn{3}{|c|}{$w \times 10^{-6}(\mathrm{~m})$} & \multicolumn{3}{c|}{$m_{x x}(\mathrm{~N} . \mathrm{m})$} & \multicolumn{3}{c|}{$\sigma_{x x}\left(\mathrm{~N} / \mathrm{m}^{2}\right)$} \\
\cline { 2 - 11 } & MEC & MEF & Dif. \% & MEC & MEF & Dif. \% & MEC & MEF & Dif. $\%$ \\
\hline 0,01 & 0,436 & 0,435 & 0,229 & 0,192 & 0,192 & 0,000 & 2890,04 & 2886,30 & 0,129 \\
\hline 0,02 & 0,055 & 0,054 & 1,851 & 0,193 & 0,193 & 0,000 & 726,30 & 726,59 & 0,03 \\
\hline 0,03 & 0,016 & 0,016 & 0,000 & 0,194 & 0,195 & 0,512 & 324,73 & 325,11 & 0,116 \\
\hline 0,04 & 0,007 & 0,007 & 0,000 & 0,196 & 0,196 & 0,000 & 183,78 & 184,07 & 0,157 \\
\hline
\end{tabular}

\subsubsection{Influência do número de elementos no contorno}

A placa (Figura 7.1) foi discretizada usando-se 20, 40, 60, 80 e 100 elementos constantes no contorno de mesmo comprimento. Foi usado somente 1 nó interno e foram obtidos resultados para deslocamento vertical, momento e tensão no centro da placa. Foram obtidos resultados para as espessuras e coeficiente de Poisson de $h=0,02 \mathrm{~m}, h=0,2 \mathrm{~m}, \nu=0,0$ e $\nu=0,3$, respectivamente. 
Tabela 7.5: Deslocamentos e esforços no centro de uma placa apoiada sujeita a um carregamento distribuído: $h=0,02 \mathrm{~m}$ e $\nu=0,0$.

\begin{tabular}{|c|c|c|c|c|c|}
\hline \multirow{2}{*}{ MALHA } & \multicolumn{2}{|c|}{ Deslocamento } & \multicolumn{2}{c|}{ Momento } & Tensão \\
\cline { 2 - 6 } & $w \times 10^{-6}(\mathrm{~m})$ & Dif. $(\%)$ & $m_{x x}(\mathrm{~N} . \mathrm{m})$ & Dif. $(\%)$ & $\sigma_{x x}\left(\mathrm{~N} / \mathrm{m}^{2}\right)$ \\
\hline 20 & $-0,512168$ & 7,797 & $-0,155257$ & 5,473 & $-2328,87$ \\
\hline 40 & $-0,488212$ & 2,755 & $-0,150152$ & 2,005 & $-2252,28$ \\
\hline 60 & $-0,483679$ & 1,801 & $-0,149155$ & 1,328 & $-2237,33$ \\
\hline 80 & $-0,481958$ & 1,039 & $-0,148771$ & 1,067 & $-2231,57$ \\
\hline 100 & $-0,481132$ & 1,260 & $-0,148586$ & 0,941 & $-2228,79$ \\
\hline 120 & $-0,480695$ & 1,172 & $-0,148488$ & 0,875 & $-2227,30$ \\
\hline 140 & $-0,480455$ & 1,122 & $-0,148434$ & 0,831 & $-2226,51$ \\
\hline 160 & $-0,480325$ & 1,095 & $-0,148404$ & 0,817 & $-2226,07$ \\
\hline 180 & $-0,480258$ & 1,080 & $-0,148390$ & 0,808 & $-2225,85$ \\
\hline 200 & $-0,480258$ & 1,080 & $-0,148384$ & 0,804 & $-2225,85$ \\
\hline 220 & $-0,480224$ & 1,073 & $-0,148383$ & 0,803 & $-2225,75$ \\
\hline 240 & $-0,480234$ & 1,075 & $-0,148386$ & 0,805 & $-2225,75$ \\
\hline 260 & $-0,480252$ & 1,079 & $-0,148390$ & 0,808 & $-2225,86$ \\
\hline Bares (1969) & $-0,475122$ & $-0,147200$ & - \\
\hline MEF & $-0,48008$ & $-0,14832$ & $-2224,8$ \\
\hline
\end{tabular}


Tabela 7.6: Deslocamentos e esforços no centro de uma placa apoiada sujeita a um carregamento distribuído: $h=0,02 \mathrm{~m}$ e $\nu=0,3$.

\begin{tabular}{|c|c|c|c|c|c|}
\hline \multirow{2}{*}{ MALHA } & \multicolumn{2}{|c|}{ Deslocamento } & \multicolumn{2}{c|}{ Momento } & Tensão \\
\cline { 2 - 6 } & $w \times 10^{-9}(\mathrm{~m})$ & Dif. $(\%)$ & $m_{x x}(\mathrm{~N} . \mathrm{m})$ & Dif. $(\%)$ & $\sigma_{x x}\left(\mathrm{~N} / \mathrm{m}^{2}\right)$ \\
\hline 20 & $-0,451545$ & 4,382 & $-0,197200$ & 2,922 & $-2958,02$ \\
\hline 40 & $-0,440081$ & 1,732 & $-0,193838$ & 1,168 & $-2907,57$ \\
\hline 60 & $-0,437915$ & 1,232 & $-0,193163$ & 0,815 & $-2897,46$ \\
\hline 80 & $-0,437054$ & 1,033 & $-0,192888$ & 0,672 & $-2893,32$ \\
\hline 100 & $-0,436621$ & 0,932 & $-0,192747$ & 0,598 & $-2891,21$ \\
\hline 120 & $-0,436382$ & 0,877 & $-0,192669$ & 0,557 & $-2890,04$ \\
\hline 140 & $-0,436243$ & 0,845 & $-0,192623$ & 0,533 & $-2889,35$ \\
\hline 160 & $-0,436161$ & 0,826 & $-0,192596$ & 0,519 & $-2888,95$ \\
\hline 180 & $-0,436113$ & 0,815 & $-0,192581$ & 0,512 & $-2888,71$ \\
\hline 200 & $-0,436086$ & 0,809 & $-0,192572$ & 0,507 & $-2888,58$ \\
\hline 220 & $-0,436073$ & 0,806 & $-0,192568$ & 0,505 & $-2888,52$ \\
\hline 240 & $-0,436068$ & 0,805 & $-0,192566$ & 0,504 & $-2888,50$ \\
\hline 260 & $-0,436069$ & 0,805 & $-0,192567$ & 0,504 & $-2888,50$ \\
\hline Bares (1969) & $-0,432585$ & $-0,191600$ & - \\
\hline MEF & $-0,43575$ & $-0,19242$ & $-2886,30$ \\
\hline
\end{tabular}

Pode-se observar nas Tabelas 7.5 e 7.6 que os deslocamentos apresentam diferenças elevadas (em torno de $7 \%$ e 4\%, respectivamente) apenas para uma malha pouco refinada, ou seja, a malha de 20 elementos no contorno ( 5 elementos por lado). Com uma malha igual a 260 elementos constantes (65 elementos por lado) chega-se a diferenças em torno de 0,8\% para deslocamentos e 0,5\% para momentos, o que de fato exige um baixo esforço computacional se comparado com outros métodos númericos (por exemplo, elementos finitos). 
Tabela 7.7: Deslocamentos e esforços no centro de uma placa apoiada sujeita a um carregamento distribuído: $h=0,2 \mathrm{~m}$ e $\nu=0,0$.

\begin{tabular}{|c|c|c|c|c|c|}
\hline \multirow{2}{*}{ MALHA } & \multicolumn{2}{|c|}{ Deslocamento } & \multicolumn{2}{c|}{ Momento } & Tensão \\
\cline { 2 - 6 } & $w \times 10^{-9}(\mathrm{~m})$ & Dif. $(\%)$ & $m_{x x}($ N.m $)$ & Dif. $(\%)$ & $\sigma_{x x}\left(\mathrm{~N} / \mathrm{m}^{2}\right)$ \\
\hline 20 & $-0,565423$ & 1,899 & $-0,163756$ & 1,164 & $-24,56$ \\
\hline 40 & $-0,548823$ & 1,092 & $-0,160342$ & 0,944 & $-24,05$ \\
\hline 60 & $-0,547985$ & 1,243 & $-0,160218$ & 1,021 & $-24,03$ \\
\hline 80 & $-0,548324$ & 1,182 & $-0,160321$ & 0,957 & $-24,04$ \\
\hline 100 & $-0,548722$ & 1,110 & $-0,160425$ & 0,893 & $-24,04$ \\
\hline 120 & $-0,549050$ & 1,051 & $-0,160508$ & 0,842 & $-24,07$ \\
\hline 140 & $-0,549306$ & 1,005 & $-0,160571$ & 0,803 & $-24,08$ \\
\hline 160 & $-0,549507$ & 0,969 & $-0,160621$ & 0,772 & $-24,09$ \\
\hline 180 & $-0,549667$ & 0,940 & $-0,160660$ & 0,748 & $-24,09$ \\
\hline 200 & $-0,549796$ & 0,917 & $-0,160692$ & 0,728 & $-24,10$ \\
\hline 220 & $-0,549901$ & 0,898 & $-0,160717$ & 0,712 & $-24,10$ \\
\hline 240 & $-0,549989$ & 0,882 & $-0,160739$ & 0,699 & $-24,11$ \\
\hline Palermo $(2000)$ & $-0,554885$ & $-0,161871$ & - \\
\hline MEF & $-0,553$ & $-0,161$ & $-24,22$ \\
\hline
\end{tabular}


Tabela 7.8: Deslocamentos e esforços no centro de uma placa apoiada sujeita a um carregamento distribuído: $h=0,2 \mathrm{~m}$ e $\nu=0,3$.

\begin{tabular}{|c|c|c|c|c|c|}
\hline \multirow{2}{*}{ MALHA } & \multicolumn{2}{|c|}{ Deslocamento } & \multicolumn{2}{c|}{ Momento } & Tensão \\
\cline { 2 - 6 } & $w \times 10^{-9}(\mathrm{~m})$ & Dif. $(\%)$ & $m_{x x}(\mathrm{~N} . \mathrm{m})$ & Dif. $(\%)$ & $\sigma_{x x}\left(\mathrm{~N} / \mathrm{m}^{2}\right)$ \\
\hline 20 & $-0,499019$ & 1,912 & $-0,205651$ & 0,422 & $-30,847$ \\
\hline 40 & $-0,490421$ & 0,156 & $-0,203232$ & 0,758 & $-30,484$ \\
\hline 60 & $-0,489618$ & 0,0073 & $-0,203027$ & 0,858 & $-30,454$ \\
\hline 80 & $-0,489547$ & 0,0218 & $-0,203023$ & 0,860 & $-30,453$ \\
\hline 100 & $-0,489589$ & 0,0132 & $-0,203046$ & 0,849 & $-30,456$ \\
\hline 120 & $-0,489643$ & 0,0022 & $-0,203069$ & 0,838 & $-30,460$ \\
\hline 140 & $-0,489690$ & 0,0073 & $-0,203087$ & 0,829 & $-30,463$ \\
\hline 160 & $-0,489729$ & 0,0153 & $-0,203102$ & 0,822 & $-30,465$ \\
\hline 180 & $-0,489760$ & 0,0216 & $-0,203114$ & 0,816 & $-30,467$ \\
\hline 200 & $-0,489785$ & 0,0267 & $-0,203123$ & 0,812 & $-30,468$ \\
\hline 220 & $-0,489805$ & 0,0308 & $-0,203130$ & 0,808 & $-30,469$ \\
\hline 240 & $-0,489822$ & 0,0343 & $-0,203137$ & 0,805 & $-30,470$ \\
\hline Palermo $(2000)$ & $-0,489654$ & $-0,204786$ & - \\
\hline MEF & $-0,49516$ & $-0,20236$ & $-30,354$ \\
\hline
\end{tabular}

Nas Tabelas 7.7 e 7.8 os resultados apresentam boa concordância com a referência, pois a formulação leva em conta o efeito da cortante ao longo da espessura, o que de fato torna os resultados mais atrativos. Pode-se notar que as diferenças nos resultados apresentaram valores em torno de 0,03\% para deslocamentos e 0,08\% para momentos. 
Para o cálculo dos momentos no contorno (lado BC) da placa apoiada (Figura 7.1) foram comparados os resultados com a condição de contorno do problema afim de validar os resultados obtidos. Para tal condição tem-se os momentos tangencial e normal ao contorno da placa, onde $M_{t} \neq 0$ e $M_{n}=0$. O interesse deste problema é mostrar os momentos tangenciais no contorno da placa. A relação entre os momentos e a normal ao ponto fonte (ver equações (5.92) e (5.93)) foi usada. Para tal problema analisamos uma placa isotrópica que apresenta as seguintes propriedades e dimensões: $E=205 \mathrm{GPa}, a=2 \mathrm{~m}$ e espessuras iguais a $h=0,2$ $\mathrm{m}$ ( $10 \%$ do lado), $h=0,3 \mathrm{~m}$ ( $15 \%$ do lado) e $h=0,4 \mathrm{~m}$ (20\% do lado). A placa apoiada é carregada por uma carga $q=1 \mathrm{~N} / \mathrm{m}^{2}$ com sentido contrário a $x_{3}$ e coeficiente de Poisson igual a 0,3 . Os resultados são mostrados na Tabela (7.9).

Tabela 7.9: Momento no lado BC da placa apoiada.

\begin{tabular}{|c|c|c|c|c|c|c|}
\hline \multirow{2}{*}{$x$} & \multicolumn{2}{|c|}{$h=0,2(\mathrm{~m})$} & \multicolumn{2}{c|}{$h=0,3(\mathrm{~m})$} & \multicolumn{2}{c|}{$h=0,4(\mathrm{~m})$} \\
\cline { 2 - 7 } & $p(\mathrm{~N} . \mathrm{m})$ & $C D C$ & $p(\mathrm{~N} . \mathrm{m})$ & $C D C$ & $p(\mathrm{~N} . \mathrm{m})$ & $C D C$ \\
\hline 0,1 & $-0,0606$ & $-0,4461$ & $-0,1093$ & $-0,3330$ & $-0,0907$ & $-0,2479$ \\
\hline 0,3 & 0,5215 & 0,5739 & 0,4745 & 0,4909 & 0,4435 & 0,4479 \\
\hline 0,5 & 0,6885 & 0,7193 & 0,6767 & 0,6991 & 0,6603 & 0,6770 \\
\hline 0,7 & 0,7761 & 0,8077 & 0,7755 & 0,8009 & 0,7665 & 0,7892 \\
\hline 0,9 & 0,8153 & 0,8458 & 0,8187 & 0,8435 & 0,8126 & 0,8363 \\
\hline 1,1 & 0,8153 & 0,8458 & 0,8187 & 0,8435 & 0,8126 & 0,8363 \\
\hline 1,3 & 0,7761 & 0,8077 & 0,7755 & 0,8009 & 0,7665 & 0,7892 \\
\hline 1,5 & 0,6885 & 0,7193 & 0,6767 & 0,6991 & 0,6603 & 0,6770 \\
\hline 1,7 & 0,5215 & 0,5739 & 0,4745 & 0,4909 & 0,4435 & 0,4479 \\
\hline 1,9 & $-0,0606$ & $-0,4461$ & $-0,1093$ & $-0,3330$ & $-0,0907$ & $-0,2479$ \\
\hline
\end{tabular}

Os resultados mostrados na Tabela (7.9) apresentam boa concordância em comparação com a condição de contorno (CDC), divergindo apenas nos extremos por causa do efeito nos cantos. 
Na Figura 7.3 foram gerados 29 nos internos e foram obtidos os valores de deslocamento vertical e momentos ao longo da linha central da placa (ver Figura 7.3). Os resultados apresentaram boa concordância com o método dos elementos finitos (MEF).

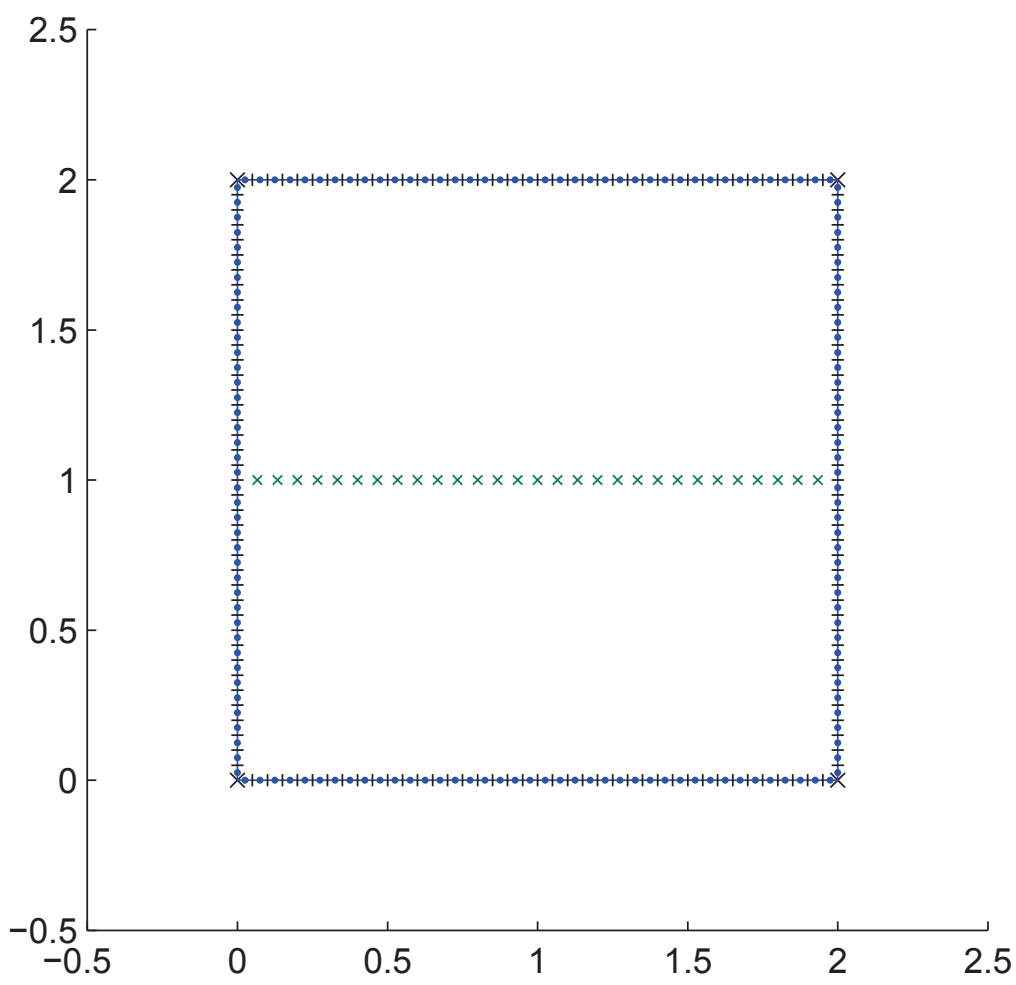

Figura 7.3: Discretização com 40 elementos no contorno e 19 nos internos. 


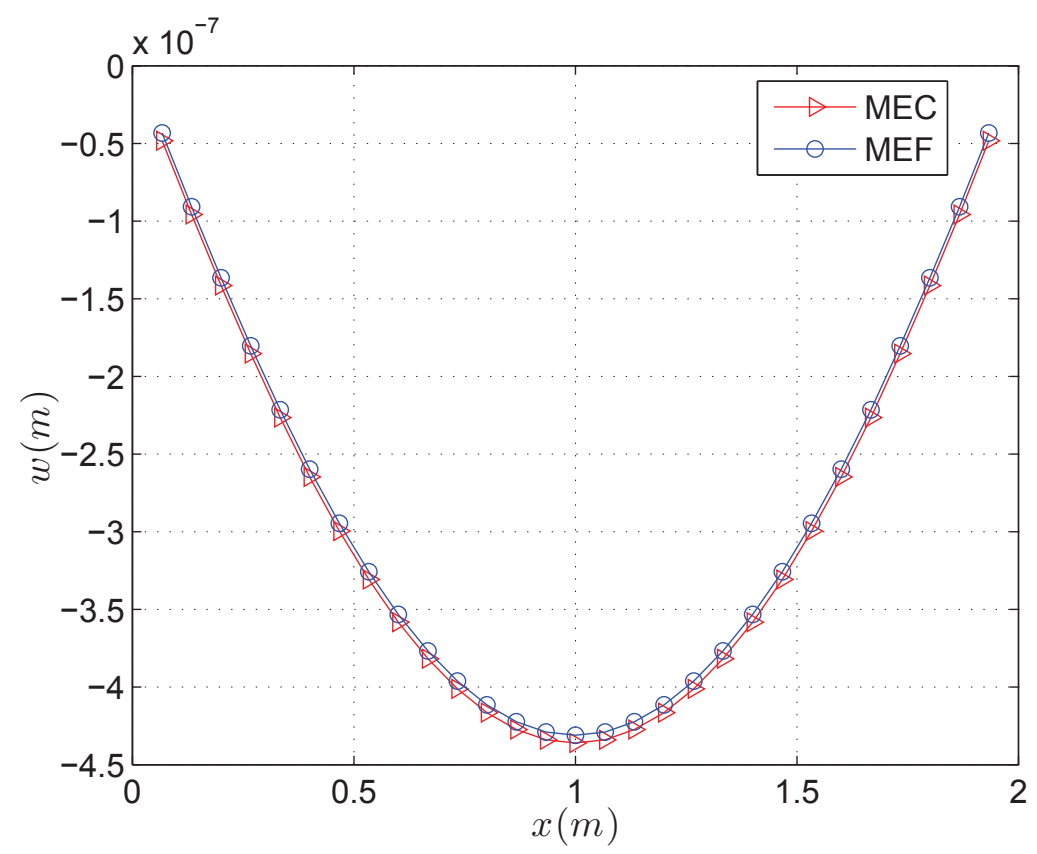

Figura 7.4: Deslocamento vertical ao longo da linha central da placa.

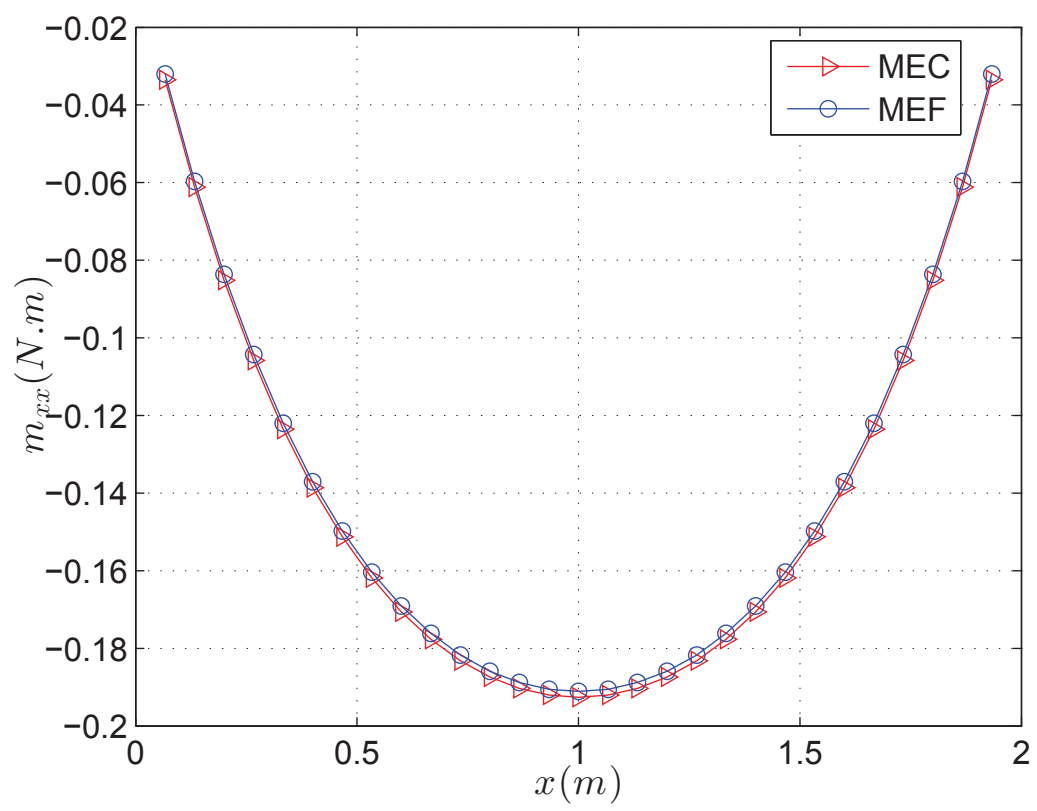

Figura 7.5: Momentos na direção $x$ ao longo da linha central da placa. 


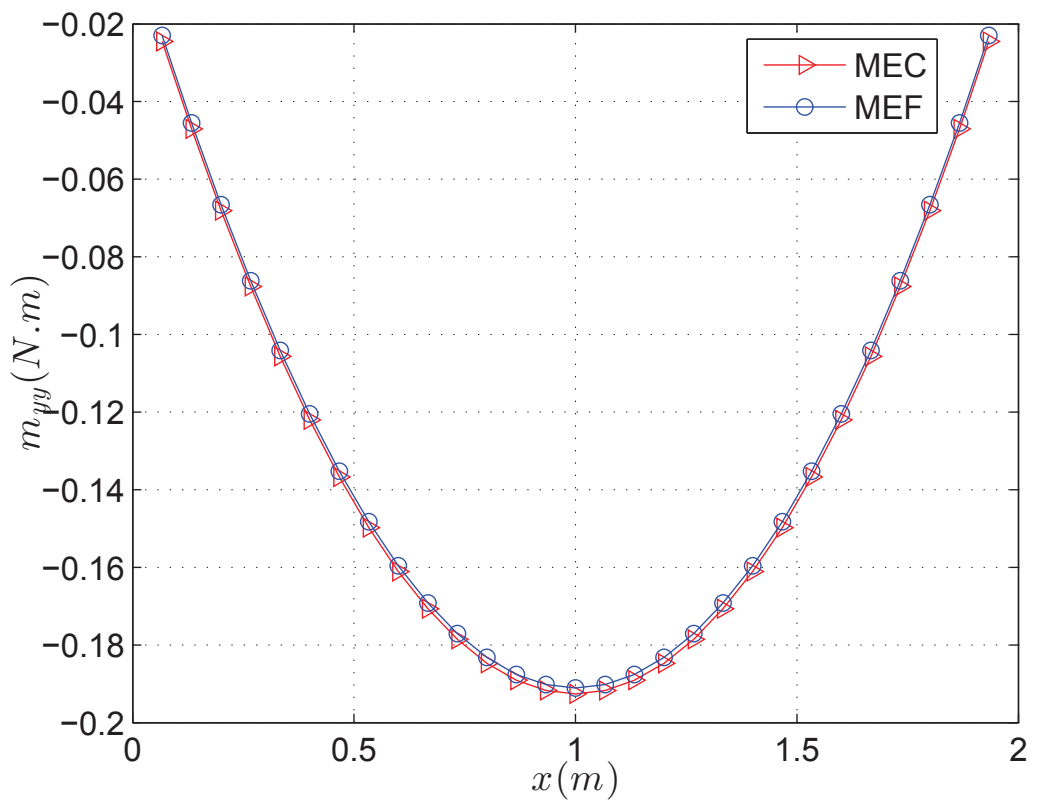

Figura 7.6: Momentos na direção y ao longo da linha central da placa.

O mapa de cor para deslocamentos do nó central na direção $x_{3}$ e momentos $\left(m_{x x}\right)$ podem ser visualizados nas Figuras 7.7 e 7.8 . 


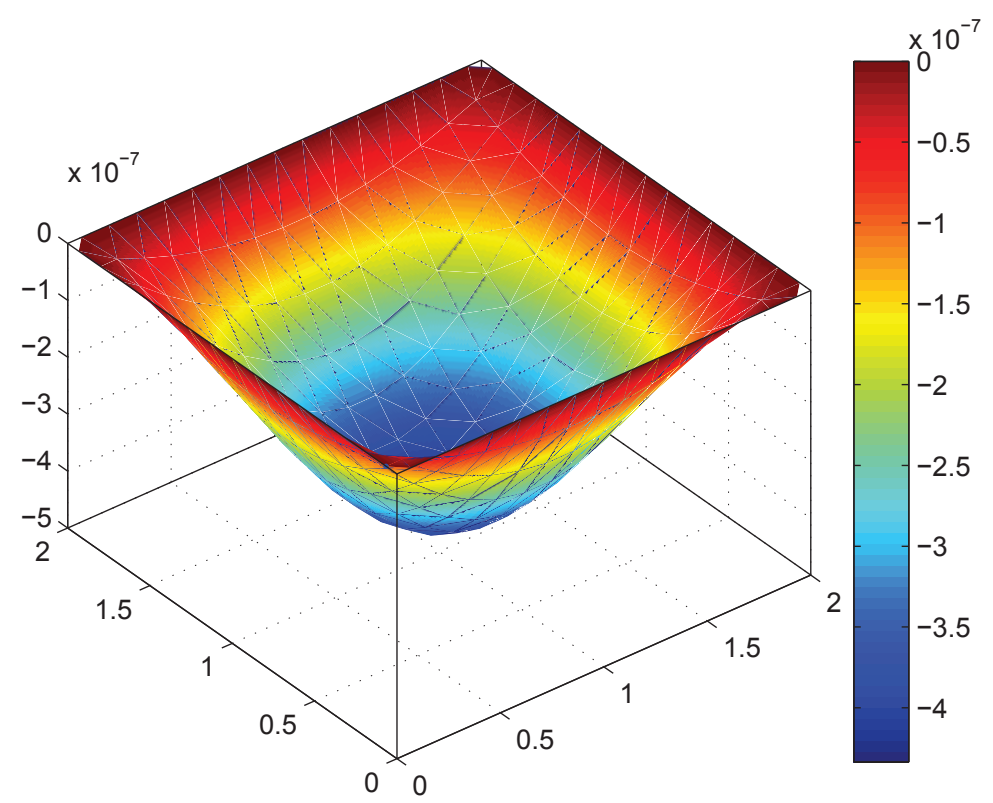

Figura 7.7: Mapa de cor do deslocamento vertical do nó central da placa de espessura $h=$ $0,02 \mathrm{~m}$.

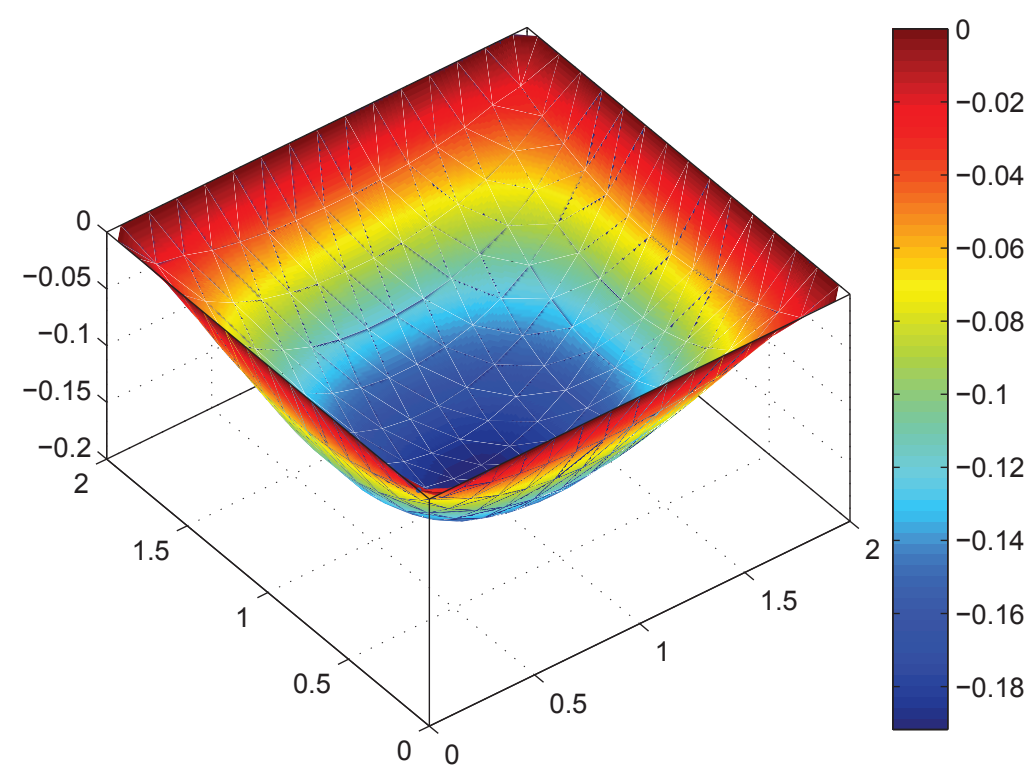

Figura 7.8: Mapa de cor do momento $\left(m_{x x}\right)$ da placa de espessura $h=0,02 \mathrm{~m}$. 


\subsubsection{Placa quadrada engastada nos quatro lados sob carga uni- formemente distribuída}

Considere uma placa engastada (Figura 7.9) carregada por uma carga $q=1 \mathrm{~N} / \mathrm{m}^{2}$ com sentido contrário a $x_{3}$. A placa é isotrópica e apresenta as seguintes propriedades e dimensões: $E=205 \mathrm{GPa}, \nu=0,3, a=2 \mathrm{~m}$ e espessuras iguais a $h=0,02 \mathrm{~m}, h=0,1 \mathrm{~m}$ e $h=0,2 \mathrm{~m}$. Este problema é equivalente ao proposto por Sanches (1998).

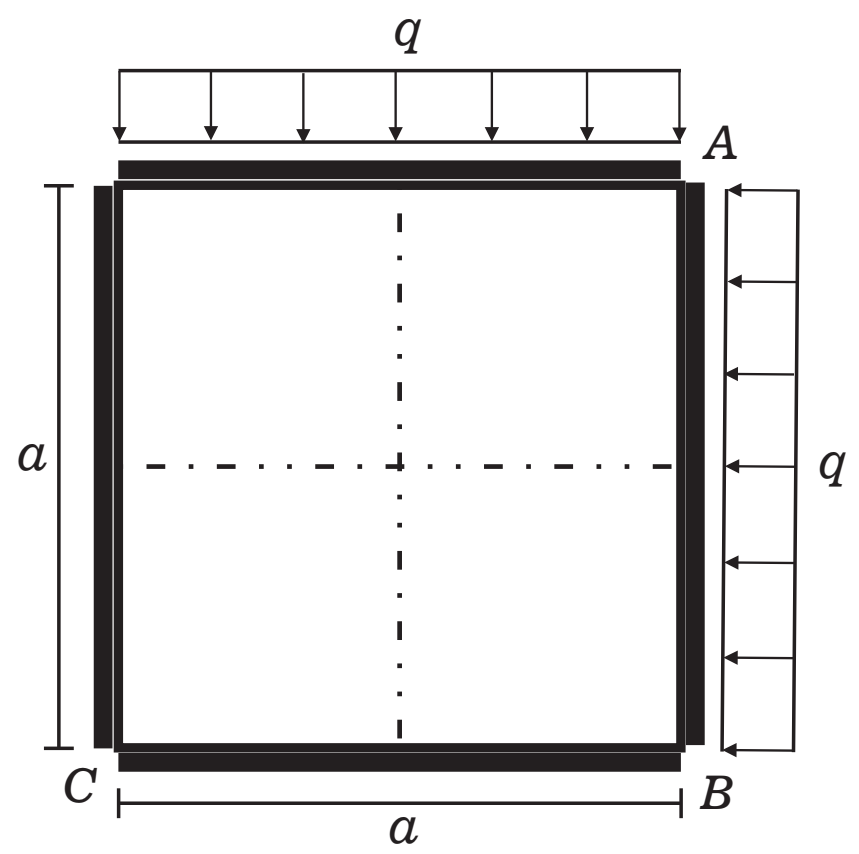

Figura 7.9: Placa quadrada engastada nos quatro lados.

Para placa engastada as seguintes condições de contorno são conhecidas ao longo dos lados da placa mostrada acima:

$$
u_{t}=0 \longleftrightarrow u_{n}=0 \longleftrightarrow u_{3}=0 \longleftrightarrow M_{t} \neq 0 \longleftrightarrow M_{n} \neq 0
$$


Tabela 7.10: Deslocamentos e esforços no centro de uma placa engastada sujeita a um carregamento distribuído.

\begin{tabular}{|c|c|c|c|c|c|c|c|}
\hline \multirow{2}{*}{$h$} & \multicolumn{3}{|c|}{$w \times 10^{-6}(\mathrm{~m})$} & \multicolumn{3}{c|}{$m_{x x}(\mathrm{~N} . \mathrm{m})$} & $\sigma_{x x}\left(\mathrm{~N} / \mathrm{m}^{2}\right)$ \\
\cline { 2 - 8 } & Sanches $(1998)$ & MEC & Dif. \% & Sanches (1998) & MEC & Dif. \% & MEC \\
\hline 0,02 & $-1014,40$ & $-1016,69$ & 0,225 & $-0,0912$ & $-0,0917$ & 0,548 & $-1376,5$ \\
\hline 0,1 & $-8,0639$ & $-8,0217$ & 0,523 & $-0,0924$ & $-0,0921$ & 0,324 & $-55,2918$ \\
\hline 0,2 & $-1,2040$ & $-1,2065$ & 0,2 & $-0,0944$ & $-0,0929$ & 1,588 & $-13,9451$ \\
\hline
\end{tabular}

A Tabela 7.10 apresenta resultados para deslocamento vertical, momentos e tensões no centro da placa engastada. Os resultados apresentam boa concordância com a literatura apresentando diferenças inferiores a $0,5 \%$.

Tabela 7.11: Deslocamentos e esforços no centro da placa para diferentes relações $h / a$.

\begin{tabular}{|c|c|c|c|c|c|c|c|c|c|}
\hline \multirow{2}{*}{$h / a$} & \multicolumn{3}{|c|}{$w \times 10^{-6}(\mathrm{~m})$} & \multicolumn{3}{c|}{$m_{x x}(\mathrm{~N} . \mathrm{m})$} & \multicolumn{3}{c|}{$\sigma_{x x}\left(\mathrm{~N} / \mathrm{m}^{2}\right)$} \\
\cline { 2 - 11 } & MEC & MEF & Dif. $\%$ & MEC & MEF & Dif. $\%$ & MEC & MEF & Dif. $\%$ \\
\hline 0,01 & 0,135 & 0,135 & 0,00 & 0,0917 & 0,0917 & 0,00 & 1375,72 & 1374,20 & 2,07 \\
\hline 0,02 & 0,01701 & 0,01690 & 0,65 & 0,09176 & 0,09179 & 0,03 & 344,12 & 344,22 & 0,03 \\
\hline 0,03 & 0,00509 & 0,00500 & 1,80 & 0,09184 & 0,09184 & 0,00 & 153,07 & 152,54 & 0,34 \\
\hline 0,04 & 0,00217 & 0,00211 & 2,84 & 0,09195 & 0,09179 & 0,17 & 86,203 & 82,056 & 0,17 \\
\hline
\end{tabular}

Tabela 7.12: Esforços no lado BC da placa para diferentes relações $h / a$.

\begin{tabular}{|c|c|c|c|c|c|c|}
\hline \multirow{2}{*}{$h / a$} & \multicolumn{3}{|c|}{$m_{x x}(\mathrm{~N} . \mathrm{m})$} & \multicolumn{3}{c|}{$\sigma_{x x}\left(\mathrm{~N} / \mathrm{m}^{2}\right)$} \\
\cline { 2 - 7 } & MEC & MEF & Dif. $\%$ & MEC & MEF & Dif. $\%$ \\
\hline 0,01 & 0,2051 & 0,2016 & 1,73 & 3076,81 & 3024,60 & 1,72 \\
\hline 0,02 & 0,2051 & 0,2036 & 0,73 & 737,14 & 738,65 & 0,20 \\
\hline 0,03 & 0,2044 & 0,2033 & 0,54 & 338,17 & 337,178 & 0,29 \\
\hline 0,04 & 0,2022 & 0,2023 & 0,04 & 189,637 & 189,663 & 0,01 \\
\hline
\end{tabular}


Nas Tabelas 7.11 e 7.12 foram obtidos valores para deslocamento vertical, momento e tensão em relação aos pontos no centro e no contorno (ponto médio do lado $A B$ ) da placa, respectivamente (ver Figura Engastada111). Nas Figuras 7.10, 7.11 e 7.12 foram obtidos os valores de deslocamento vertical, momento e tensões ao longo da linha central da placa (ver Figura 7.3). Os resultados foram comparados com o métodos dos elementos finitos (MEF) e apresentaram boa concordância.

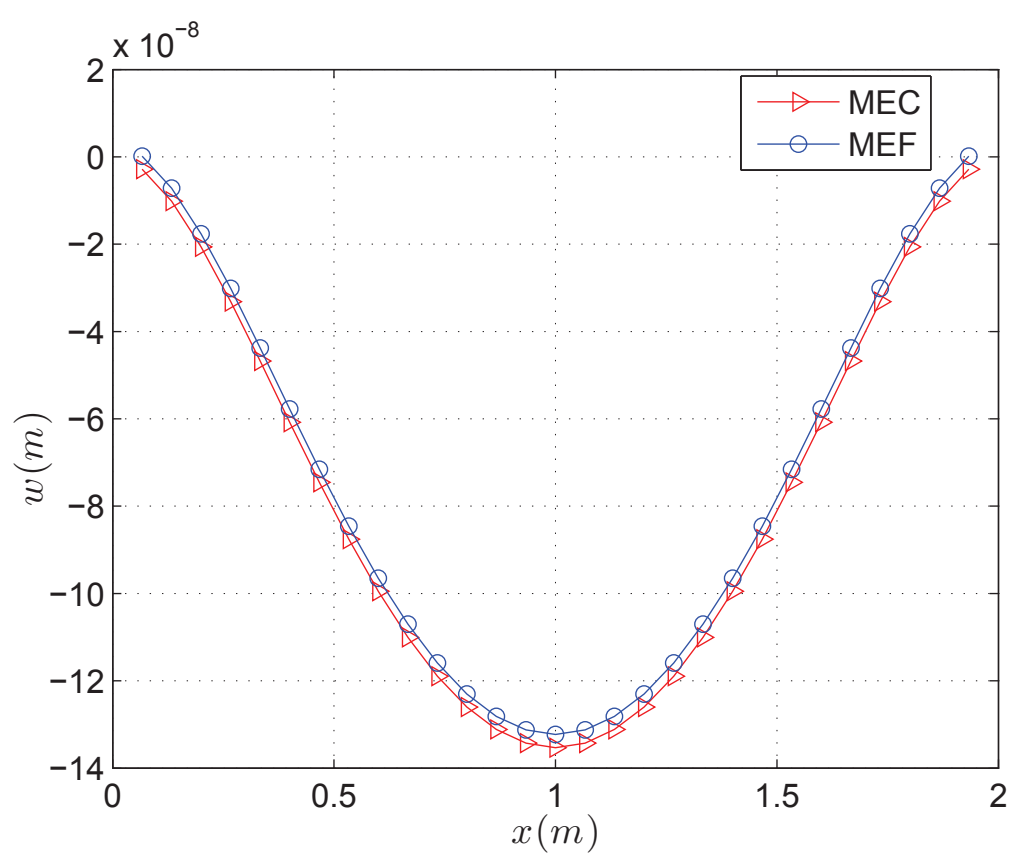

Figura 7.10: Deslocamentos na direção $w$ ao longo da linha central da placa. 


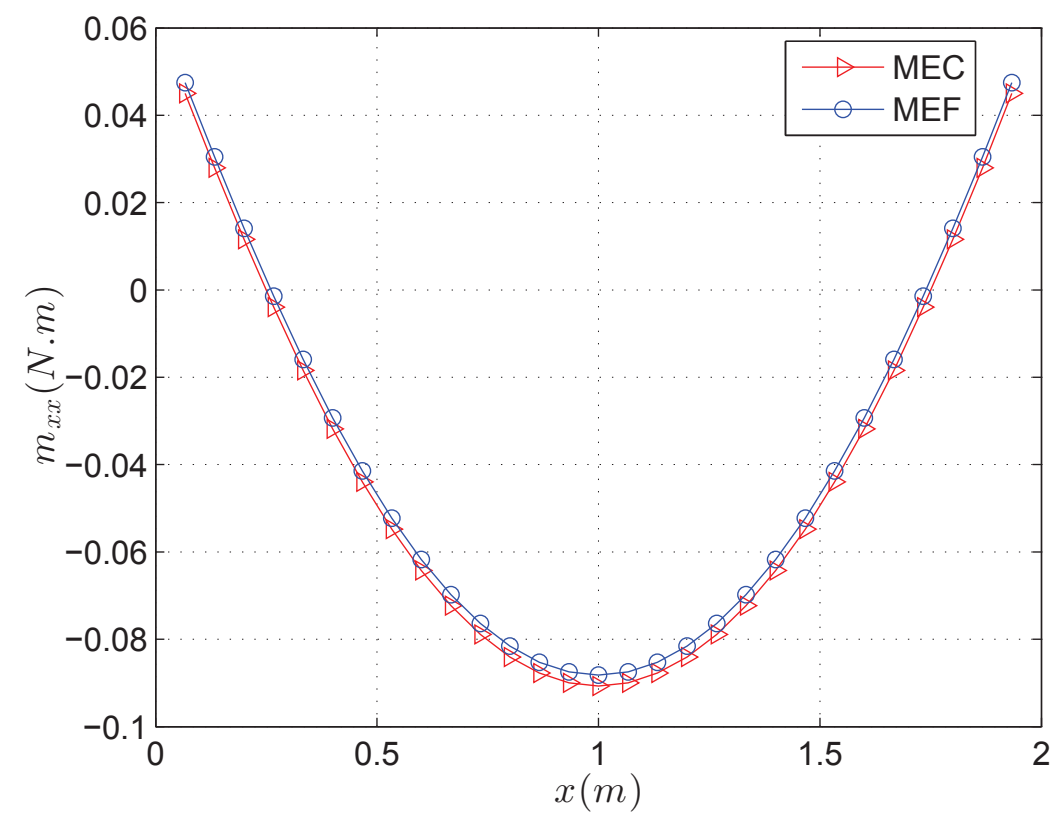

Figura 7.11: Momentos na direção $x$ ao longo da linha central da placa.

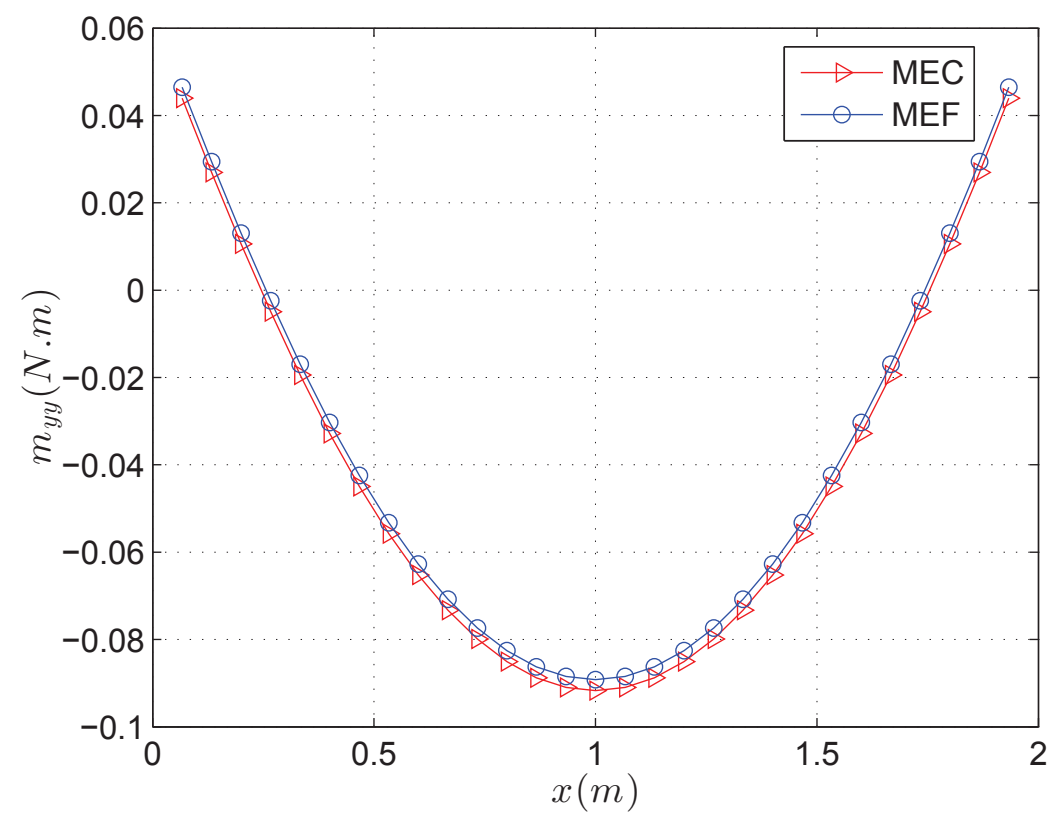

Figura 7.12: Momentos na direção y ao longo da linha central da placa.

Nas Figuras 9.13 e 9.14 foram obtidos resultados para momentos ao longo do contorno $A B$ da Figura 7.9. Os resultados apresentaram boa concordância com o método dos elementos 
finitos (MEF).

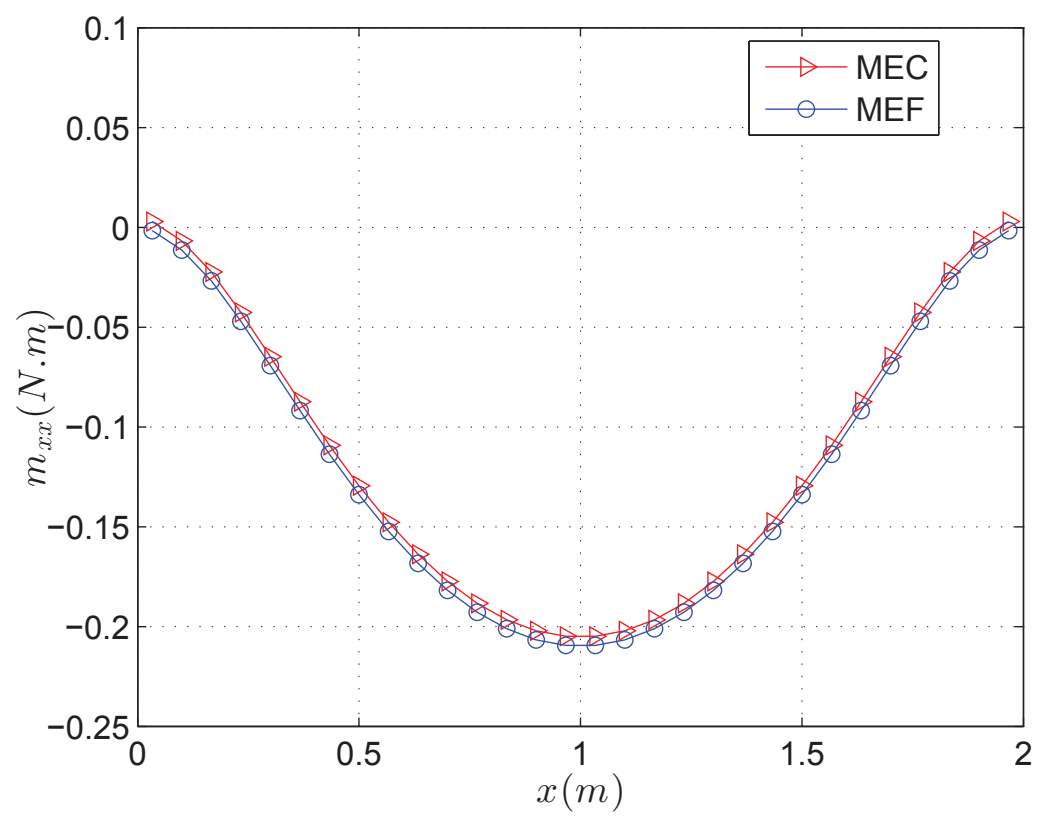

Figura 7.13: Momentos na direção $x$ ao longo do lado $A B$ da placa.

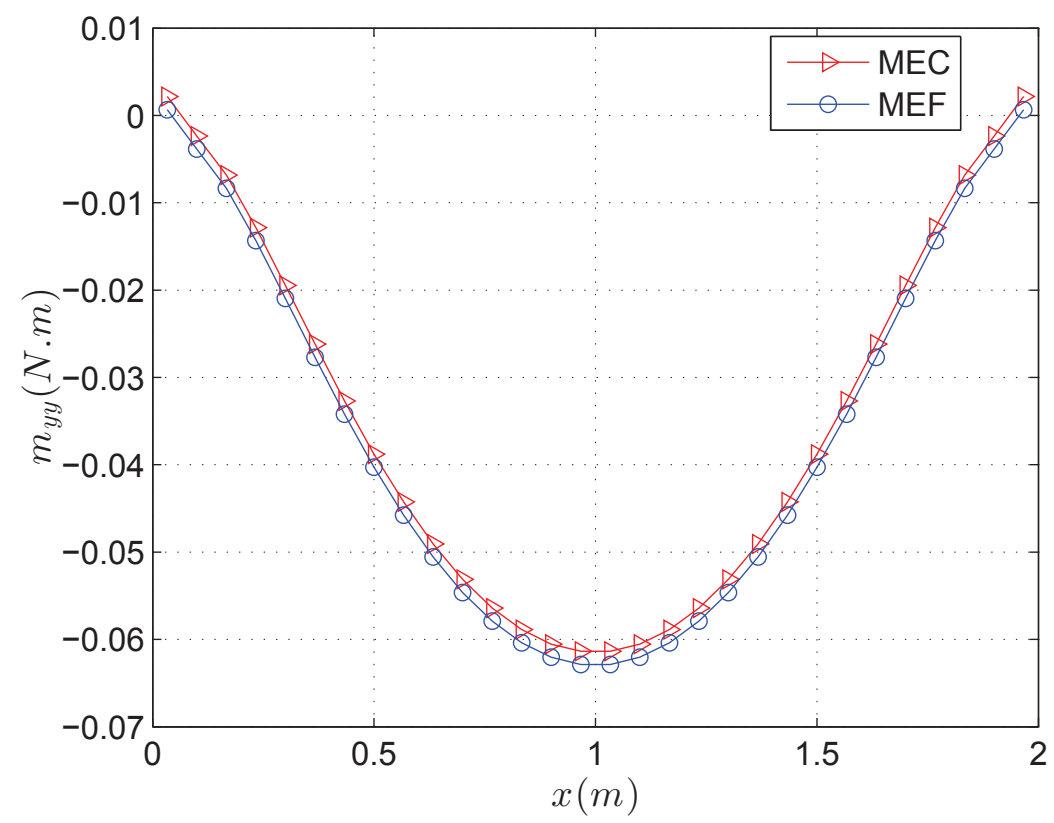

Figura 7.14: Momentos na direção $y$ ao longo do lado $A B$ da placa. 


\subsubsection{Influência do número de elementos no contorno}

A placa (Figura 7.9) foi discretizada usando-se 20, 40, 60, 80 e 100 elementos no contorno, respectivamente.

Tabela 7.13: Deslocamentos e esforços no centro de uma placa engastada sujeita a um carregamento distribuído: $h=0,02 \mathrm{~m}$ e $\nu=0,3$.

\begin{tabular}{|c|c|c|c|c|c|}
\hline \multirow{2}{*}{ MALHA } & \multicolumn{2}{|c|}{ Deslocamento } & \multicolumn{2}{c|}{ Momento } & Tensões \\
\cline { 2 - 6 } & $w\left(\times 10^{-6} \mathrm{~m}\right)$ & Dif. $(\%)$ & $m_{x x}(\mathrm{~N} . \mathrm{m})$ & Dif. $(\%)$ & $\sigma_{x x}\left(\mathrm{~N} / \mathrm{m}^{2}\right)$ \\
\hline 20 & $-0,136082$ & 1,054 & $-0,091973$ & 0,847 & $-1379,598$ \\
\hline 40 & $-0,135466$ & 0,597 & $-0,091772$ & 0,627 & $-1376,592$ \\
\hline 60 & $-0,135383$ & 0,535 & $-0,091744$ & 0,596 & $-1376,167$ \\
\hline 80 & $-0,135346$ & 0,507 & $-0,091732$ & 0,583 & $-1375,981$ \\
\hline 100 & $-0,135325$ & 0,492 & $-0,091724$ & 0,574 & $-1375,874$ \\
\hline 120 & $-0,135312$ & 0,482 & $-0,091720$ & 0,570 & $-1375,806$ \\
\hline 140 & $-0,135302$ & 0,475 & $-0,091717$ & 0,566 & $-1375,757$ \\
\hline 160 & $-0,135295$ & 0,470 & $-0,091714$ & 0,563 & $-1375,721$ \\
\hline 180 & $-0,135290$ & 0,466 & $-0,091712$ & 0,561 & $-1375,694$ \\
\hline 200 & $-0,135286$ & 0,463 & $-0,091711$ & 0,560 & $-1375,672$ \\
\hline 220 & $-0,135282$ & 0,460 & $-0,091710$ & 0,559 & $-1375,654$ \\
\hline 240 & $-0,135279$ & 0,458 & $-0,091709$ & 0,558 & $-1375,639$ \\
\hline 260 & $-0,135277$ & 0,456 & $-0,091708$ & 0,557 & $-1375,626$ \\
\hline Sanches (1998) & $-0,134662$ & \multicolumn{5}{|c|}{$-0,0912$} & - \\
\hline MEF & $-0,135$ & \multicolumn{5}{|c|}{$-0,0916$} & $-1375,00$ \\
\hline
\end{tabular}

Da Tabela 7.13, nota-se que as diferenças nos resultados diminuem à medida que o número de nós aumenta no contorno. As diferenças são pouco significativas e comportam-se em torno de $0,5 \%$ e 0,6\% para deslocamentos e momentos, respectivamente à medida que o número de nós no contorno aumenta.

O mapa de cor para deslocamentos do nó central na direção $x_{3}$ e momentos $\left(m_{x x}\right)$ 
podem ser visualizados nas Figuras 7.15 e 7.16 .

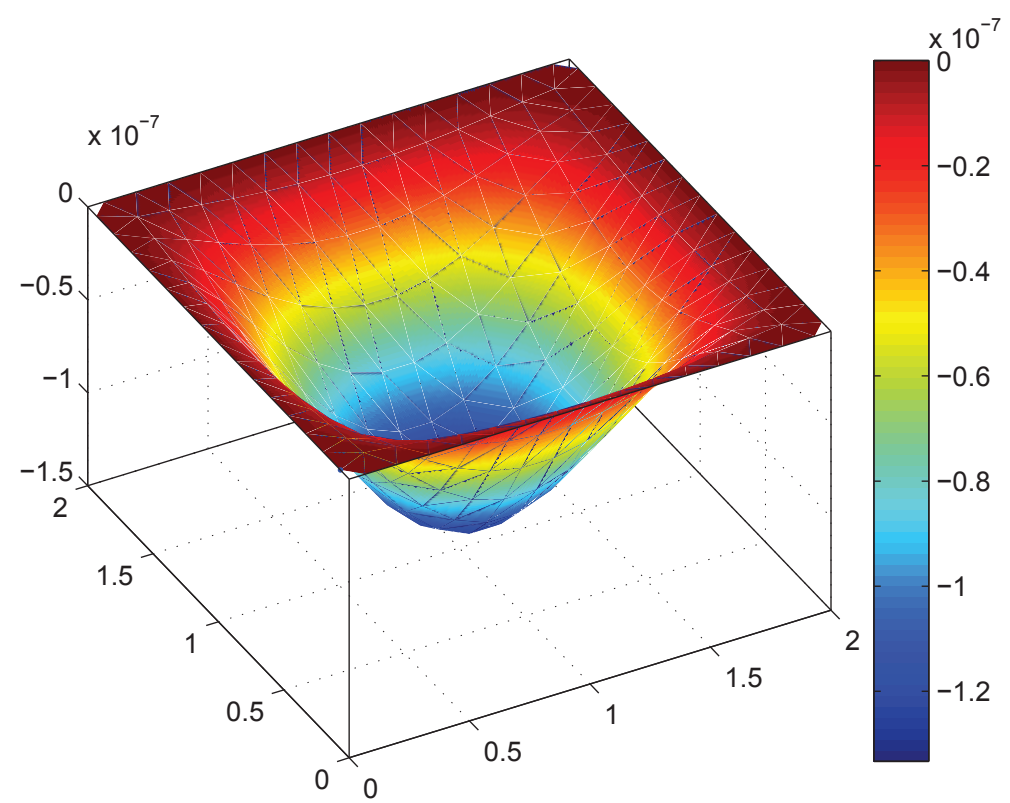

Figura 7.15: Mapa de cor do deslocamento vertical do nó central da placa de espessura $h=0,02 \mathrm{~m}$.

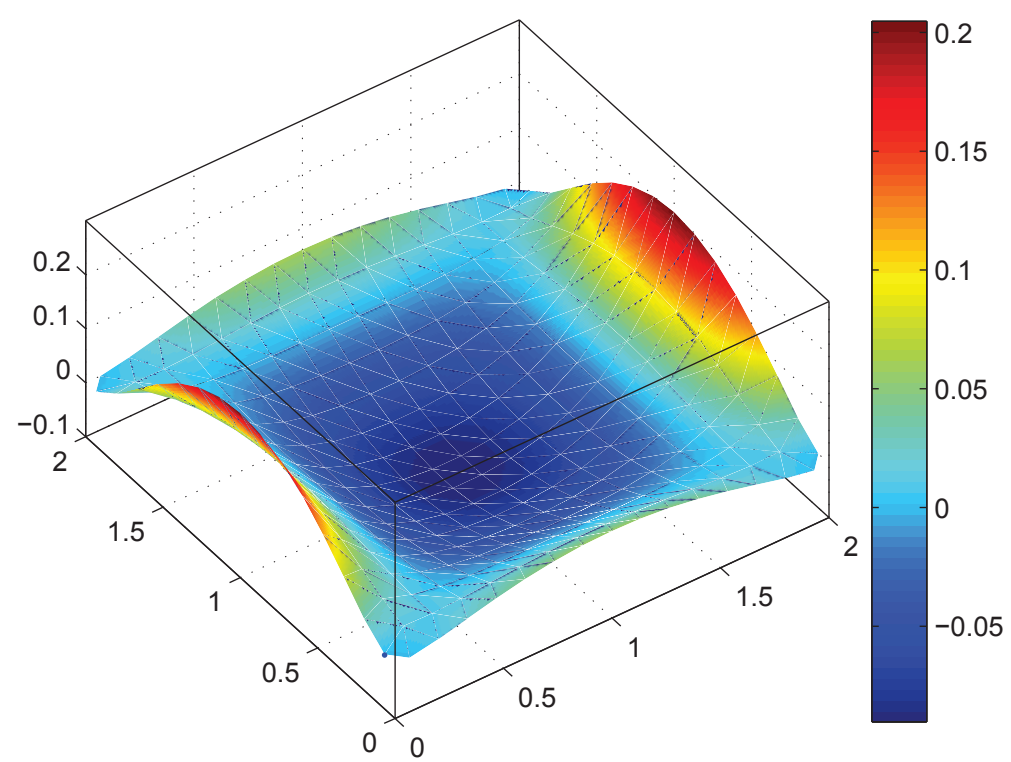

Figura 7.16: Mapa de cor do momento $\left(m_{x x}\right)$ da placa de espessura $h=0,02 \mathrm{~m}$. 


\subsubsection{Placa quadrada com um lado engastado e três lados apoiados sob carga uniformemente distribuída}

Considere uma placa engastada-apoiada (Figura 7.17) carregada por uma carga $q=1$ $\mathrm{N} / \mathrm{m}^{2}$ com sentido contrário a $x_{3}$. A placa é isotrópica e apresenta as mesmas propriedades e dimensões da seção 7.1.1. Este problema é equivalente ao proposto por Palermo (2000).

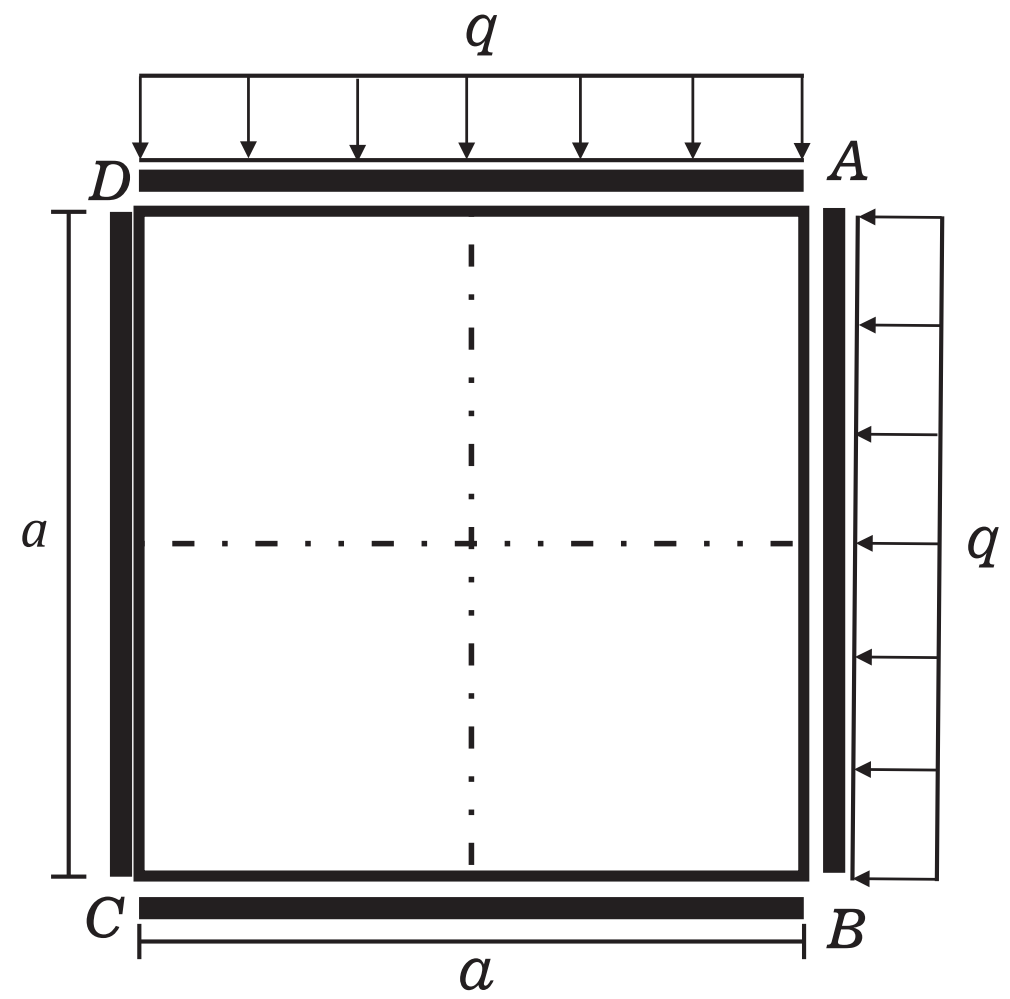

Figura 7.17: Placa quadrada com um lado engastado e os outros três apoiados. 
A apresentação dos resultados é análoga a do exemplo anterior e segue uma comparação dos resultados referentes à distribuição dos esforços no centro da placa (Figura 7.17). Podese notar nas Tabela 7.14 que as diferenças nos resultados diminuem com o aumento da malha tanto para deslocamento quanto para momento. Não foram encontrados na literatura resultados para a força cortante. Na Tabela 7.15 as diferenças apresentam-se estabilizados tanto para deslocamentos quanto para momentos, não havendo muita variação nos resultados com o refinamento da malha.

Tabela 7.14: Deslocamentos e esforços no centro de uma placa engastada-apoiada sujeita a um carregamento distribuído: $h=0,02 \mathrm{~m}$ e $\nu=0,3$.

\begin{tabular}{|c|c|c|c|c|}
\hline \multirow{2}{*}{ MALHA } & \multicolumn{2}{|c|}{ Deslocamento } & \multicolumn{2}{c|}{ Momento } \\
\cline { 2 - 5 } & $w\left(\times 10^{-7} \mathrm{~m}\right)$ & Dif. (\%) & $m_{x x}$ (N.m) & Dif. (\%) \\
\hline 20 & $-3,050$ & 2,693 & $-0,1593$ & 1,594 \\
\hline 40 & $-3,002$ & 1,104 & $-0,1579$ & 0,701 \\
\hline 60 & $-2,993$ & 0,786 & $-0,1575$ & 0,446 \\
\hline 80 & $-2,989$ & 0,652 & $-0,1574$ & 0,382 \\
\hline 100 & $-2,987$ & 0,582 & $-0,1573$ & 0,318 \\
\hline 120 & $-2,986$ & 0,538 & $-0,1573$ & 0,318 \\
\hline 140 & $-2,985$ & 0,505 & $-0,1572$ & 0,255 \\
\hline 160 & $-2,984$ & 0,471 & $-0,1572$ & 0,255 \\
\hline 180 & $-2,984$ & 0,471 & $-0,1572$ & 0,255 \\
\hline Palermo (2000) & \multicolumn{3}{|c|}{$-2,970$} & \multicolumn{2}{c|}{} \\
\hline
\end{tabular}


Tabela 7.15: Deslocamentos e esforços no centro de uma placa engastada-apoiada sujeita a um carregamento distribuído: $h=0,2 \mathrm{~m}$ e $\nu=0,3$.

\begin{tabular}{|c|c|c|c|c|}
\hline \multirow{2}{*}{ MALHA } & \multicolumn{2}{|c|}{ Deslocamento } & \multicolumn{2}{c|}{ Momento } \\
\cline { 2 - 5 } & $w\left(\times 10^{-10} \mathrm{~m}\right)$ & Dif. (\%) & $m_{x x}($ N.m) & Dif. (\%) \\
\hline 20 & $-3,43904$ & 0,351 & $-0,163551$ & 1,058 \\
\hline 40 & $-3,40623$ & 0,606 & $-0,162709$ & 1,567 \\
\hline 60 & $-3,40440$ & 0,659 & $-0,162708$ & 1,568 \\
\hline 80 & $-3,40469$ & 0,651 & $-0,162743$ & 1,546 \\
\hline 100 & $-3,40511$ & 0,638 & $-0,162770$ & 1,530 \\
\hline 120 & $-3,40545$ & 0,628 & $-0,162789$ & 1,519 \\
\hline 140 & $-3,40570$ & 0,621 & $-0,162802$ & 1,511 \\
\hline 160 & $-3,40588$ & 0,616 & $-0,162812$ & 1,505 \\
\hline 180 & $-3,40601$ & 0,612 & $-0,162815$ & 1,501 \\
\hline 200 & $-3,40611$ & 0,609 & $-0,162826$ & 1,500 \\
\hline 220 & $-3,40619$ & 0,607 & $-0,162828$ & 1,595 \\
\hline 240 & $-3,40625$ & 0,605 & $-0,162831$ & 1,493 \\
\hline Palermo (2000) & $-3,427$ & \multicolumn{2}{c}{$-0,1653$} \\
\hline
\end{tabular}


Tabela 7.16: Deslocamentos e esforços ao longo do lado $C D$ da placa engastada-apoiada sujeita a um carregamento distribuído: $h=0,2 \mathrm{~m}$.

\begin{tabular}{|c|c|c|c|c|c|c|}
\hline \multirow{2}{*}{ x/a } & \multicolumn{3}{|c|}{ Poisson $\nu=0,0$} & \multicolumn{3}{c|}{ Poisson $\nu=0,3$} \\
\cline { 2 - 7 } & Palermo (N.m) & $m_{x x}$ (N.m) & Dif. (\%) & Palermo (N.m) & $m_{x x}$ (N.m) & Dif. (\%) \\
\hline 0 & $-0,0061$ & - & - & $-0,0079$ & - & - \\
\hline 0,1 & $-0,1328$ & $-0,1335$ & 0,527 & $-0,1325$ & $-0,1315$ & 0,754 \\
\hline 0,2 & $-0,2264$ & $-0,2224$ & 1,766 & $-0,2263$ & $-0,2258$ & 0,220 \\
\hline 0,3 & $-0,2895$ & $-0,2979$ & 2,901 & $-0,2894$ & $-0,2896$ & 0,069 \\
\hline 0,4 & $-0,3260$ & $-0,3299$ & 1,196 & $-0,3258$ & $-0,3212$ & 1,411 \\
\hline 0,5 & $-0,3379$ & $-0,3404$ & 0,7398 & $-0,3377$ & $-0,3315$ & 1,835 \\
\hline 0,6 & $-0,3260$ & $-0,3299$ & 1,196 & $-0,3258$ & $-0,3212$ & 1,411 \\
\hline 0,7 & $-0,2895$ & $-0,2979$ & 2,901 & $-0,2894$ & $-0,2896$ & 0,069 \\
\hline 0,8 & $-0,2264$ & $-0,2224$ & 1,766 & $-0,2263$ & $-0,2258$ & 0,220 \\
\hline 0,9 & $-0,1328$ & $-0,1335$ & 0,527 & $-0,1325$ & $-0,1315$ & 0,754 \\
\hline 1 & $-0,0061$ & - & - & $-0,0079$ & - & - \\
\hline
\end{tabular}

A Tabela 7.16 mostra a distribuição de momentos ao longo do lado engastado CD (Figura 7.17). Nota-se que as diferenças nos resultados apresentam-se inferiores a $2 \%$. Não foram obtidos resultados nos cantos 0 e 1, pois foram usados elementos constantes na discretização.

O mapa de cor para deslocamentos do nó central na direção $x_{3}$ e momentos $\left(m_{x x}\right)$ podem ser visualizados nas Figuras 7.18 e 7.19. 


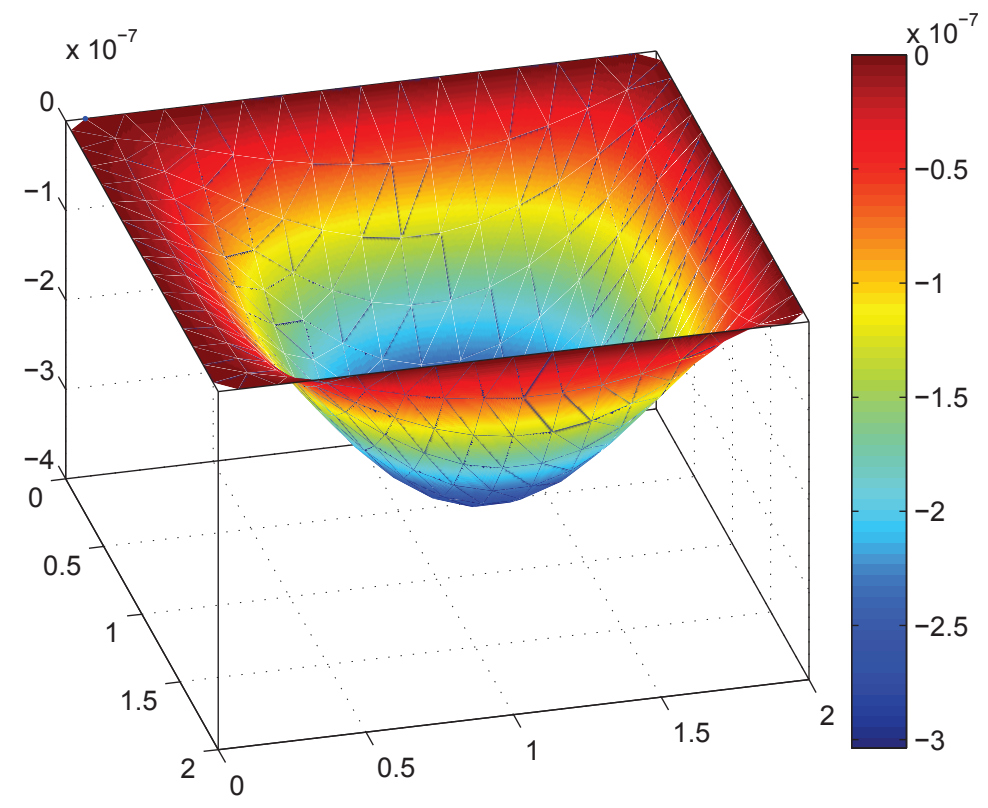

Figura 7.18: Mapa de cor do deslocamento vertical do nó central da placa de espessura $h=0,02 \mathrm{~m}$.

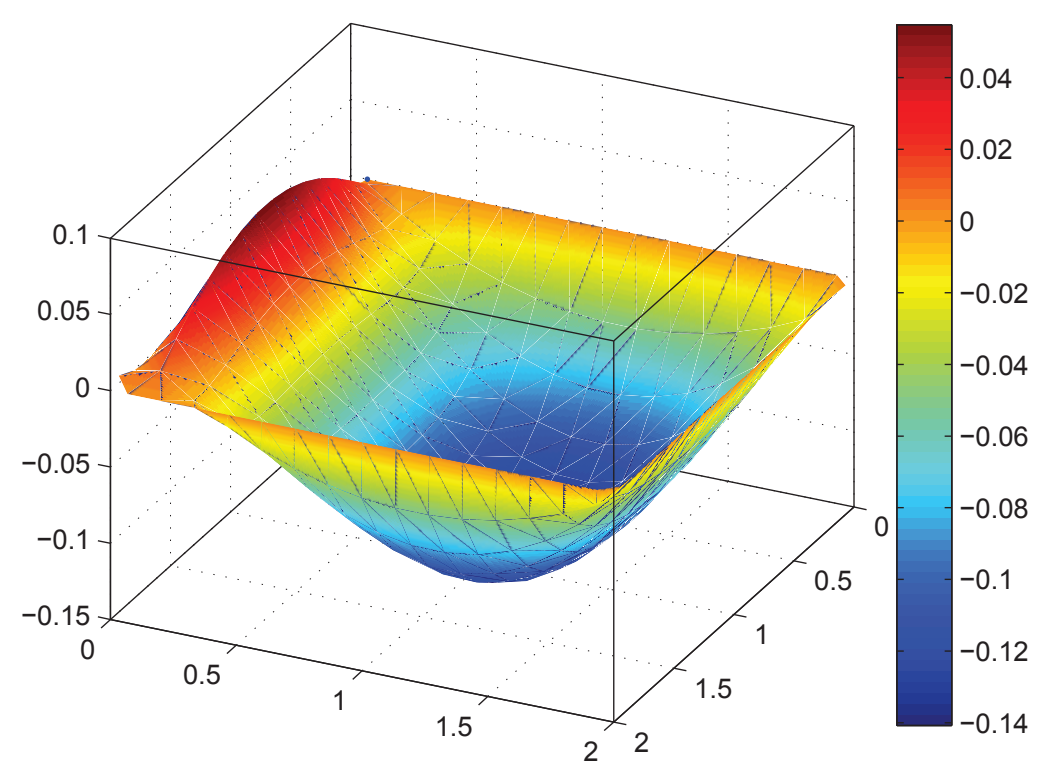

Figura 7.19: Mapa de cor do momento $\left(m_{x x}\right)$ da placa de espessura $h=0,02 \mathrm{~m}$. 


\section{Capítulo 8}

\section{Placas espessas ortotrópicas}

\subsection{Introdução}

Neste Capítulo é desenvolvida a formulação dos elementos de contorno para placas espessas ortotrópicas. A solução fundamental ortotrópica, que leva em conta o efeito do cisalhamento transversal, é obtida usando-se o operador Hörmander e a transformada de Radon. As integrais de domínio provenientes das forças de corpo são transformadas em integrais de contorno usando o método dos elementos de contorno de integração radial (MIR).

\subsection{Equações básicas}

O tensor das tensões é dado pela equação (4.1). As componentes dos deslocamentos são dadas pela equação (4.2), as deformações lineares de flexão pela equação (4.3) e deformações angulares pela equação (4.4). A lei de Hooke toma a forma que resulta da consideração de ortotropia do material com a seguinte forma (Dinis, 2004): 


$$
\begin{gathered}
\left\{\begin{array}{c}
\sigma_{11} \\
\sigma_{22} \\
\sigma_{12}
\end{array}\right\}=\left[\begin{array}{ccc}
E_{11} & E_{12} & 0 \\
E_{12} & E_{22} & 0 \\
0 & 0 & E_{33}
\end{array}\right]\left\{\begin{array}{l}
\varepsilon_{11} \\
\varepsilon_{22} \\
\varepsilon_{12}
\end{array}\right\} \\
\left\{\begin{array}{l}
\sigma_{13} \\
\sigma_{23}
\end{array}\right\}=\left[\begin{array}{cc}
E_{44} & 0 \\
0 & E_{55}
\end{array}\right]\left\{\begin{array}{c}
\gamma_{13} \\
\gamma_{23}
\end{array}\right\}
\end{gathered}
$$

onde $E_{i j}$ são as constantes do material segundo os eixos materiais da placa e que são definidas em termos dos módulos de Young e dos coeficientes de Poisson da seguinte forma:

$$
\begin{array}{lll}
E_{11}=E_{1} /\left(1-\nu_{12} \nu_{21}\right) & E_{12}=\nu_{12} E_{2} /\left(1-\nu_{12} \nu_{21}\right) & E_{22}=E_{2} /\left(1-\nu_{12} \nu_{21}\right) \\
E_{22}=G_{12} & E_{44}=G_{23} & E_{55}=G_{13}
\end{array}
$$

Substituindo as equações (8.3) e as equações (4.3) e (4.4) e admitindo que há coincidência entre os eixos materiais e os eixos da placa, as relações entre as tensões, deslocamentos e as rotações são as seguintes:

$$
\begin{gathered}
\sigma_{11}=-\frac{x_{3}}{\left(1-\nu_{12} \nu_{21}\right)}\left(E_{1} \frac{\partial \theta_{1}}{\partial x_{1}}+\nu_{12} E_{2} \frac{\partial \theta_{2}}{\partial x_{2}}\right) \\
\sigma_{22}=-\frac{x_{3}}{\left(1-\nu_{12} \nu_{21}\right)}\left(E_{2} \frac{\partial \theta_{2}}{\partial x_{2}}+\nu_{12} E_{1} \frac{\partial \theta_{1}}{\partial x_{1}}\right) \\
\sigma_{12}=-x_{3} G_{12}\left(\frac{\partial \theta_{1}}{\partial x_{2}}+\frac{\partial \theta_{2}}{\partial x_{1}}\right) \\
\sigma_{13}=G_{13}\left(-\theta_{1}+\frac{\partial \omega}{\partial x_{1}}\right)
\end{gathered}
$$




$$
\sigma_{23}=G_{23}\left(-\theta_{2}+\frac{\partial \omega}{\partial x_{2}}\right)
$$

Os esforços generalizados definidos pelas equações (4.16) e (4.18) e levando-se em conta que as tensões são dadas pela equação (8.4), obtém-se:

$$
\left\{\begin{array}{l}
M_{11} \\
M_{22} \\
M_{12}
\end{array}\right\}=\left[\begin{array}{ccc}
D_{11} & \nu_{12} D_{22} & 0 \\
\nu_{12} D_{22} & D_{22} & 0 \\
0 & 0 & D_{33}
\end{array}\right]\left\{\begin{array}{l}
\chi_{11} \\
\chi_{22} \\
\chi_{12}
\end{array}\right\}
$$

onde

$$
\begin{array}{crrl}
D_{1}=E_{1} h^{3} / 12\left(1-\nu_{x y} \nu_{y x}\right) & D_{2}=E_{2} h^{2} / 12\left(1-\nu_{x y} \nu_{y x}\right) & D_{k} & =G_{12} h^{3} / 12 \\
C_{1}=G_{z x} k h & C_{2}=G_{z y} k h & D_{1} \nu_{y x} & =D_{2} \nu_{x y}
\end{array}
$$

Em que $k=5 / 6$ na teoria de Reissner, $E_{x}$ e $E_{y}$ são os módulos de Young, $G_{x y}, G_{z x}$ e $G_{z y}$ são os módulos de cisalhamento, $\nu_{x y}$ e $\nu_{y x}$ são as constantes de Poisson.

\subsection{Equação diferencial de equilíbrio}

Através da equação (4.37) e da equação (8.5), obtém-se:

$$
\begin{gathered}
{\left[D_{1} \frac{\partial^{2} \theta_{1}}{\partial x_{1}^{2}}+D_{k} \frac{\partial^{2} \theta_{2}}{\partial x_{2}^{2}}+\left(\nu_{x y} D_{1}+D_{k}\right) \frac{\partial^{2} \theta_{2}}{\partial x_{1} \partial x_{2}}\right]-C_{1}\left(\theta_{1}+\frac{\partial \omega}{\partial x_{1}}\right)=0} \\
{\left[\left(\nu_{x y} D_{1}+D_{k}\right) \frac{\partial^{2} \theta_{1}}{\partial x_{1} \partial x_{2}}+D_{k} \frac{\partial^{2} \theta_{2}}{\partial x_{1}^{2}}+D_{2} \frac{\partial^{2} \theta_{2}}{\partial x_{2}^{2}}\right]-C_{2}\left(\theta_{2}+\frac{\partial \omega}{\partial x_{2}}\right)=0} \\
C_{1}\left(\frac{\partial^{2} \omega}{\partial x_{1}^{2}}+\frac{\partial \theta_{1}}{\partial x_{1}}\right)+C_{2}\left(\frac{\partial^{2} \omega}{\partial x_{2}^{2}}-\frac{\partial \theta_{2}}{\partial x_{2}}\right)+q=0
\end{gathered}
$$


As equações (8.7), (8.8) e (8.9) podem ser escritas em notação indicial da seguinte forma:

$$
\Delta_{i j}^{*} U_{j}+b_{i}=0
$$

onde $b_{i}$ representa 0,0 e $q$, respectivamente, e $\Delta_{i j}^{*}$ são operadores diferenciais que podem ser escritos como:

$$
\begin{array}{ll}
\Delta_{11}^{*}=D_{1} \frac{\partial^{2}}{\partial x_{1}^{2}}+D_{k} \frac{\partial^{2}}{\partial x_{2}^{2}}-C_{1} & \Delta_{22}^{*}=D_{1} \frac{\partial^{2}}{\partial x_{1}^{2}}+D_{k} \frac{\partial^{2}}{\partial x_{2}^{2}}-C_{2} \\
\Delta_{12}^{*}=\Delta_{21}^{*}=\left(D_{1} \mu_{y x}+D_{k}\right) \frac{\partial^{2}}{\partial x_{1} \partial x_{2}} & \Delta_{13}^{*}=\Delta_{31}^{*}=-C_{1} \frac{\partial}{\partial x_{1}} \\
\Delta_{23}^{*}=-\Delta_{32}^{*}=-C_{2} \frac{\partial}{\partial x_{2}} & \Delta_{33}^{*}=C_{1} \frac{\partial^{2}}{\partial x_{1}^{2}}+C_{2} \frac{\partial^{2}}{\partial x_{2}^{2}}
\end{array}
$$

\subsection{Relação entre forças generalizadas $\left(M_{\alpha \beta}\right.$ e $\left.Q_{\alpha}\right)$ e des- locamentos $(\Psi$ e $W)$}

Neste trabalho é assumido que o eixo principal ortotrópico coincide com os eixos $x_{1}$ e $x_{2}$. $U_{i}$ representa os deslocamentos da placa, $U_{\alpha}$ são as rotações $\psi_{x}$ e $\psi_{y}$, e $U_{3}$ é a deflexão na direção da espessura. Para as equações seguintes usa-se índices gregos com valores 1 e 2 , e índices latinos com valores 1, 2 e 3. Quando nada ao contrário for dito, índices repetidos indicam soma de acordo com a convenção de Einstein.

1. Relação entre os esforços (cortantes e momentos) e os deslocamentos são dados por:

$$
\begin{gathered}
M_{\alpha \beta}=D_{\alpha \beta}\left(\psi_{\alpha, \beta}+\psi_{\beta, \alpha}\right)+C_{\alpha \beta} \psi_{\gamma, \gamma} \\
Q_{\alpha}=C_{\alpha}\left(W_{, \alpha}+\psi_{\alpha}\right)
\end{gathered}
$$

onde não se aplica a convenção de soma para os índices $\alpha$ e $\beta$. As constantes presentes nas equações (8.12) e (8.13) são dadas por: 


$$
\begin{array}{ll}
D_{11}=\frac{D_{1}}{2}\left(1-\mu_{y x}\right) & D_{22}=\frac{D_{2}}{2}\left(1-\mu_{x y}\right) \\
D_{12}=D_{21}=D_{k} & C_{11}=D_{1} \mu_{y x} \\
C_{22}=D_{2} \mu x y & C_{12}=C_{21}=0
\end{array}
$$

\subsection{Forças e deslocamentos no contorno $\Gamma$}

Se $n_{\alpha}$ e $t_{\alpha}$ representam os vetores unitários normal e tangente ao contorno $\Gamma$ da placa, respectivamente, as forças e deslocamentos no contorno $\Gamma$ podem ser expressas como:

$$
\begin{array}{ll}
P_{\alpha}=M_{\alpha \beta} n_{\beta} & P_{3}=Q_{\alpha} n_{\alpha} \\
M_{n}=M_{\alpha \beta} n_{\alpha} n_{\beta} & M_{n s}=M_{\alpha \beta} t_{\alpha} n_{\beta} \\
\psi_{n}=\psi_{\alpha} n_{\alpha} & \psi_{s}=\psi_{\alpha} n_{\alpha}
\end{array}
$$

\subsection{Solução fundamental}

A solução fundamental para placas ortotrópicas espessas levando em consideração o efeito do cisalhamento transversal é uma solução particular da equação (8.10) devido a uma carga unitária concentrada, ou seja, a solução satisfaz a seguinte equação diferencial nãohomogênea (Wang, 1991):

$$
\Delta_{i j}^{*} U_{k j}^{*}(\zeta, x)=-\delta(\zeta, x) \delta_{k i}
$$

em que $\delta(\zeta, x)$ denota da função delta de Dirac, $\zeta$ representa o ponto campo, e $x$ é o ponto fonte. Seguindo o método do operador de Hor̈mander (ver Apêndice A), a solução da equação (8.16) pode ser escrita como: 


$$
U_{k j}^{*}(\zeta, x)=\mathbf{C o} \Delta_{j k}^{*} \Phi(\zeta, x)
$$

onde $\Phi$ é uma função escalar e $\mathbf{C o} \Delta^{*}$ representa a matriz cofator de $\Delta^{*}$ e são dados por:

$$
\begin{gathered}
{ }^{c o} \Delta_{\alpha \beta}^{*}=E_{\alpha \beta} \nabla^{2} \nabla_{k}^{2}-B_{\alpha \beta} \frac{\partial^{2}}{\partial x_{\alpha} \partial x_{\beta}} \nabla_{k}^{2}-C_{1} C_{2} \frac{\partial^{2}}{\partial x_{\alpha} \partial x_{\beta}} \\
{ }^{c o} \Delta_{3 \alpha}^{*}=-\mathbf{C o} \Delta_{\alpha 3}^{*}=\frac{\partial}{\partial x_{\alpha}}\left(E_{\alpha 3} \frac{\partial^{2}}{\partial x_{2}^{2}}+B_{\alpha 3} \frac{\partial^{2}}{\partial x_{1}^{2}}-C_{1} C_{2}\right) \\
{ }^{c o} \Delta_{33}^{*}=D_{1} D_{k} \frac{\partial^{4}}{\partial x_{1}^{4}}+\left(D_{1} D_{2}-D_{1}^{2} \mu_{y x}^{2}-2 D_{1} D_{k} \mu_{y x}\right) \frac{\partial^{4}}{\partial x_{1}^{2} \partial x_{2}^{2}} \\
+D_{2} D_{k} \frac{\partial^{4}}{\partial x_{2}^{4}}-\left(D_{1} C_{2}+C_{1} D_{k}\right) \frac{\partial^{2}}{\partial x_{1}^{2}}-\left(C_{1} D_{2}+C_{2} D_{k}\right) \frac{\partial^{2}}{\partial x_{2}^{2}}+C_{1} C_{2}
\end{gathered}
$$

onde não se aplica a convenção de soma para $\alpha$ e $\beta$. Nas equações (8.18), (8.19) e (8.20), as constantes são dadas por:

$$
\begin{array}{lrr}
E_{11}=D_{2} & E_{22}=D_{1} & E_{12}=E_{21}=0 \\
B_{11}=D_{2}-D_{k} & B_{22}=D_{1}-D_{k} & B_{12}=B_{21}=\left(D_{1} \mu_{y x}+D_{k}\right) \\
E_{13}=C_{1} D_{2}-C_{2}\left(D_{1} \mu_{y x}+D_{k}\right) & E_{23}=C_{2} D_{k} & \\
B_{23}=C_{2} D_{1}-C_{1}\left(D_{1} \mu_{y x}+D_{k}\right) & B_{13}=C_{1} D_{k} & \\
\nabla_{k}^{2}=C_{1} \frac{\partial^{2}}{\partial x_{1}^{2}}+C_{2} \frac{\partial^{2}}{\partial x_{2}^{2}} & \nabla^{2}=\frac{\partial^{2}}{\partial x_{1}^{2}}+\frac{\partial^{2}}{\partial x_{12}^{2}} &
\end{array}
$$

Por substituição da equação (8.17) na equação (8.16) e usando as equações (8.18) à (8.20), obtém-se:

$$
\left\{\nabla_{k}^{2}\left[D_{1} D_{k} \frac{\partial^{4}}{\partial x_{1}^{4}}+\left(D_{1} D_{2}-D_{1}^{2} \mu_{y x}^{2}-2 D_{1} D_{k} \mu_{y x}\right) \frac{\partial^{4}}{\partial x_{1}^{2} \partial x_{2}^{2}}+D_{2} D_{k} \frac{\partial^{4}}{\partial x_{2}^{4}}\right]\right.
$$




$$
\begin{array}{r}
-C_{1} C_{2}\left[D_{1} \frac{\partial^{2}}{\partial x_{1}^{2}}+2\left(2 D_{k}+D_{1} \mu_{y x}\right) \frac{\partial^{4}}{\partial x_{1}^{2} \partial x_{2}^{2}}\right. \\
\left.\left.+D_{2} \frac{\partial^{4}}{\partial x_{2}^{4}}\right]\right\} \Phi(\zeta, x)=-\delta(\zeta, x)
\end{array}
$$

Através do procedimento acima, a dedução da solução fundamental, dado por um sistema de equações diferenciais descrito pela equação (8.16), é reduzida para uma equação diferencial parcial, equação (8.22). A equação (8.22) é uma equação diferencial parcial de sexta ordem que pode ser reduzida a uma equação diferencial ordinária através da transformada de Radon (ver Apêndice B). Aplicamos a transformada de Radon no lado direito da equação (8.22), obtém-se:

$$
\delta(\zeta, x)=-\frac{1}{4 \pi^{2}} \int_{0}^{2 \pi}\left|\omega_{1}(x-\xi)+\omega_{2}(y-\eta)\right|^{-2} d \theta
$$

em que $\left(\omega_{1}, \omega_{2}\right)$ são coordenadas de um ponto num circulo unitário, ou seja, $\omega_{1}=\cos (\theta)$, $\omega_{2}=\sin (\theta),(x, y)$ e $(\xi, \eta)$ são coordenadas dos pontos fonte e campo, respectivamente. De forma similar, $\Phi(\zeta, x)$ pode ser escrita como:

$$
\Phi(\zeta, x)=\int_{0}^{2 \pi} \varphi(\rho) d \theta
$$

onde

$$
\rho=\omega_{1}(x-\xi)+\omega_{2}(y-\eta)
$$

ou seja, $\varphi(\rho)$ é uma função que depende somente de $\rho$. Aplicando a transformada de Radon no lado esquerdo da equação (8.22), e levando em consideração a seguinte relação diferencial $\frac{\partial}{\partial x_{\alpha}}=\omega_{\alpha} \frac{d}{d \rho}$, obtém-se:

$$
\frac{d^{4}}{d \rho^{4}}\left(\frac{d^{2}}{d \rho^{2}}-p^{2}\right) \varphi(\rho)=\frac{1}{4 \pi^{2} a^{2}}|\rho|^{2}
$$


em que,

$$
\begin{gathered}
a^{2}=C_{1} D_{1} D_{k} \omega_{1}^{6}+C_{1}\left(D_{1} D_{2}-D_{1}^{2} \mu_{y x}^{2}-2 D_{1} D_{k} \mu_{y x}\right) \omega_{1}^{4} \omega_{2}^{2} \\
+C_{1} D_{2} D_{k} \omega_{1}^{2} \omega_{2}^{4}+C_{2} D_{1} D_{k} \omega_{1}^{4} \omega_{2}^{2}+C_{2} D_{2} D_{k} \omega_{2}^{6}+C_{2}\left(D_{1} D_{2}\right. \\
\left.-D_{1}^{2} \mu_{y x}^{2}-2 D_{1} D_{k} \mu_{y x}\right) \omega_{1}^{2} \omega_{2}^{4} \\
b^{2}=C_{1} C_{2}\left[D_{1} \omega_{1}^{4}+2\left(2 D_{k}+D_{1} \mu_{y x}\right) \omega_{1}^{2} \omega_{2}^{2}+D_{2} \omega_{2}^{4}\right] \\
p^{2}=b^{2} / a^{2}
\end{gathered}
$$

A equação (8.22) é reduzida a equação (8.25). Após quatro integrações sucessivas da equação (8.25), obtém-se:

$$
\begin{gathered}
\frac{d^{4}}{d \rho^{4}}\left(\frac{d^{2}}{d \rho^{2}}-p^{2}\right) \varphi(\rho)=-\frac{1}{4 \pi^{2} a^{2}}|\rho|^{-1} \\
\frac{d^{2}}{d \rho^{2}}\left(\frac{d^{2}}{d \rho^{2}}-p^{2}\right) \varphi(\rho)=-\frac{\log [\rho]}{4 a^{2} \pi^{2}} \\
\frac{d}{d \rho}\left(\frac{d^{2}}{d \rho^{2}}-p^{2}\right) \varphi(\rho)=-\frac{-\rho+\rho \log [\rho]}{4 a^{2} \pi^{2}} \\
\left(\frac{d^{2}}{d \rho^{2}}-p^{2}\right) \varphi(\rho)=\frac{3 \rho^{2}}{16 a^{2} \pi^{2}}-\frac{\rho^{2} \log [\rho]}{8 a^{2} \pi^{2}}
\end{gathered}
$$

Assim, desprezando o primeiro termo do lado direito da equação (8.26), obtém-se: 


$$
\left(\frac{d^{2}}{d \rho^{2}}-p^{2}\right) \varphi(\rho)=-\frac{\rho^{2} \log (\rho)}{8 a^{2} \pi^{2}}
$$

A solução homogênea da equação (8.27) pode ser escrita como:

$$
\varphi(\rho)=\mu_{1} \exp ^{(p \rho)}+\mu_{2} \exp ^{(-p \rho)}
$$

As derivadas de $\varphi$ são dadas por:

$$
\begin{gathered}
\dot{\varphi}=\dot{\mu}_{1} \exp ^{(p \rho)}+\mu_{1} p \exp ^{(p \rho)}+\dot{\mu}_{2} \exp ^{(-p \rho)}+\mu_{2}(-p) \exp ^{(-p \rho)} \\
\dot{\varphi}=\dot{\mu_{1}} \exp ^{(p \rho)}+\mu_{1} p \exp ^{(p \rho)}+\dot{\mu}_{2} \exp ^{(-p \rho)}-\mu_{2} p \exp ^{(-p \rho)}
\end{gathered}
$$

onde o ponto sobre a variável representa a derivada desta variação em relação a $\rho$. Logo, obtém-se:

$$
\dot{\mu_{1}} \exp ^{(p \rho)}+\dot{\mu_{2}} \exp ^{(-p \rho)}=0
$$

Assim, a equação (8.30) torna-se:

$$
\dot{\varphi}=p \exp ^{(p \rho)} \mu_{1}-p \exp ^{(-p \rho)} \mu_{2}
$$

Fazendo a derivada segunda da equação (8.32), obtém-se:

$$
\begin{gathered}
\ddot{\varphi}=p\left(\mu_{1} \exp ^{(p \rho)}\right)-p\left(\mu_{2} \exp ^{(-p \rho)}\right) \\
\ddot{\varphi}=p\left(\dot{\mu}_{1} \exp ^{(p \rho)}+\mu_{1} p \exp ^{(p \rho)}\right)-p\left(\dot{\mu}_{2} \exp ^{(-p \rho)}+\mu_{2}(-p) \exp ^{(-p \rho)}\right)
\end{gathered}
$$




$$
\ddot{\varphi}=\dot{\mu}_{1} p \exp ^{(p \rho)}+\mu_{1} p^{2} \exp ^{(p \rho)}-\dot{\mu}_{2} p \exp ^{(-p \rho)}+\mu_{2} p^{2} \exp ^{(-p \rho)}
$$

Substituindo as equações (8.28) e (8.35) na equação (8.27), obtém-se:

$\dot{\mu_{1}} p \exp ^{(p \rho)}+\mu_{1} p^{2} \exp ^{(p \rho)}-\dot{\mu}_{2} p \exp ^{(-p \rho)}+\mu_{2} p^{2} \exp ^{(-p \rho)}-p^{2}\left(\mu_{1} \exp ^{(p \rho)}+\mu_{2} \exp ^{(-p \rho)}\right)=-\frac{p^{2} \log (\rho)}{8 a^{2} \pi^{2}}(8.36)$

Cancelando os termos $\mu_{1}$ e $\mu_{2}$, obtém-se:

$$
\dot{\mu_{1}} p \exp ^{(p \rho)}-\dot{\mu_{2}} p \exp ^{(-p \rho)}=-\frac{p^{2} \log (\rho)}{8 \pi^{2} a^{2}}
$$

Das equações (8.31) e (8.37), obtém-se o seguinte sistema:

$$
\left\{\begin{array}{ccc}
\dot{\mu}_{1} \exp ^{(p \rho)}+\dot{\mu}_{2} \exp ^{(-p \rho)} & =0 \\
\dot{\mu_{1}} p \exp ^{(p \rho)}-\dot{\mu}_{2} p \exp ^{(-p \rho)} & = & -\frac{p^{2} \log (\rho)}{8 a^{2} \pi^{2}}
\end{array}\right.
$$

Resolvendo a equação acima através da regra de Cramer, obtém-se:

$$
\left[\begin{array}{cc}
\exp ^{(p \rho)} & \exp ^{-(p \rho)} \\
p \exp ^{(p \rho)} & -p \exp ^{(-p \rho)}
\end{array}\right]\left[\begin{array}{c}
\dot{\mu_{1}} \\
\dot{\mu_{2}}
\end{array}\right]=\left[\begin{array}{c}
0 \\
-\frac{p^{2} \log (\rho)}{8 a^{2} \pi^{2}}
\end{array}\right]
$$

Calculando o determinante da matriz acima, obtém-se:

$$
\begin{gathered}
\Delta \mu=\exp ^{(p \rho)}\left(-p \exp ^{(-p \rho)}\right)-\left(p \exp ^{(p \rho) \exp ^{(-p \rho)}}\right) \\
\Delta \mu=-p \exp ^{0}-p \exp ^{0} \\
\Delta \mu=-2 p
\end{gathered}
$$


Agora calculando $\Delta \dot{\mu}_{1}$, obtém-se:

$$
\Delta \dot{\mu}_{1}=\left(\begin{array}{cc}
0 & \exp ^{(-p \rho)} \\
-\frac{p^{2} \log (\rho)}{8 a^{2} \pi^{2}} & -p \exp ^{(-p \rho)}
\end{array}\right)
$$

Logo, obtém-se:

$$
\begin{gathered}
\Delta \dot{\mu_{1}}=-\frac{p^{2} \log (\rho) \exp ^{(-p \rho)}}{8 a^{2} \pi^{2}} \\
\dot{\mu_{1}}=\frac{\Delta \dot{u_{1}}}{\Delta u} \\
\dot{\mu_{1}}=-\frac{p^{2} \log (\rho) e^{(-p \rho)}}{16 a^{2} \pi^{2}}
\end{gathered}
$$

A derivada $\dot{\mu}_{1}$ pode ser escrita como:

$$
\begin{gathered}
\dot{\mu}_{1}=\frac{d \mu_{1}}{d \rho} \rightarrow d \mu_{1}=\dot{\mu}_{1} d \rho \\
\mu_{1}=\int \frac{-p^{2} \log (\rho) \exp ^{(-p \rho)}}{16 p \pi^{2} a^{2}} d \rho
\end{gathered}
$$

Com base no valor da integração de $\mu_{1}$, obtém-se:

$$
\mu_{1}=\frac{\exp ^{(-p \rho)}\left(3+p \rho-2 \exp ^{(p \rho)} \exp \int E i^{(-p \rho)}+\left(2+2 p \rho+p^{2} \rho^{2}\right) \log (\rho)\right)}{16 a^{2} p^{4} \pi^{2}}
$$

Calculando agora o $\Delta \dot{\mu}_{2}$, obtém-se:

$$
\Delta \dot{\mu}_{2}=\left(\begin{array}{cc}
\exp ^{(p \rho)} & 0 \\
p \exp ^{(p \rho)} & -\frac{p^{2} \log (\rho)}{8 a^{2} \pi^{2}}
\end{array}\right)
$$


Logo, obtém-se:

$$
\begin{gathered}
\Delta \dot{\mu}_{2}=-\frac{p^{2} \log (\rho) \exp ^{(-p \rho)}}{8 a^{2} \pi^{2}} \\
\dot{\mu_{2}}=\frac{\Delta \dot{\mu_{2}}}{\Delta \mu} \\
\dot{\mu_{2}}=-\frac{p^{2} \log (\rho) \exp ^{(p \rho)}}{16 a^{2} \pi^{2}}
\end{gathered}
$$

A derivada $\dot{\mu}_{2}$ pode ser escrita como:

$$
\begin{gathered}
\dot{\mu_{2}}=\frac{d \mu_{2}}{d \rho} \rightarrow d \mu_{2}=\dot{\mu_{2}} d \rho \\
\mu_{2}=\int \frac{p^{2} \log (\rho) \exp ^{(p \rho)}}{16 p \pi^{2} a^{2}} d \rho
\end{gathered}
$$

Com base no valor da integração de $\mu_{2}$, obtém-se:

$$
\mu_{2}=\frac{-2 \exp \int E i^{(p \rho)}+\exp ^{(p \rho)}\left(3-p \rho+\left(2-2 p \rho+p^{2} \rho^{2}\right) \log (\rho)\right)}{16 a^{2} p^{4} \pi^{2}}
$$

Por substituição das funções $\mu_{1}$ e $\mu_{2}$ na equação (8.28), obtém-se:

$$
\begin{aligned}
& \varphi(\rho)=\frac{1}{8 \pi^{2} p^{4} a^{2}}\left[p^{2} \rho^{2} \log |\rho|+2 \log |\rho|+3\right. \\
& \left.+\exp (p \rho) \int_{\rho}^{\infty} \frac{\exp (-p \sigma)}{\sigma} d \sigma-\exp (-p \rho) \int_{-\infty}^{\rho} \frac{\exp (p \sigma)}{\sigma} d \sigma\right]
\end{aligned}
$$

Substituindo a equação (8.43) na equação (8.24) e integrando, obtém-se a função $\Phi(\zeta, x)$. Os deslocamentos generalizados e os esforços no contorno podem ser escritos da seguinte forma: 


$$
\begin{aligned}
& U_{i j}^{*}(\zeta, x)=\int_{0}^{2 \pi} \tilde{U}_{i j}(\rho) d \theta \\
& P_{i j}^{*}(\zeta, x)=\int_{0}^{2 \pi} \tilde{P}_{i j}(\rho) d \theta
\end{aligned}
$$

Das equações $(8.12),(8.13),(8.15)$ e $(8.17)$, pode-se facilmente obter $\tilde{U}_{i j}(\rho)$ e $\tilde{P}_{i j}(\rho)$ :

$$
\begin{gathered}
\tilde{U}_{\alpha \beta}(\rho)=a_{\alpha \beta} \frac{d^{4} \varphi}{d \rho^{4}}-C_{1} C_{2} \omega_{\alpha} \omega_{\beta} \frac{d^{2} \varphi}{d \rho^{2}} \\
\tilde{U}_{3 \alpha}(\rho)=-\tilde{U}_{\alpha 3}(\rho)=f_{\alpha} \frac{d^{3} \varphi}{d \rho^{3}}-C_{1} C_{2} \omega_{\alpha} \frac{d \varphi}{d \rho} \\
\tilde{U}_{33}(\rho)=\alpha_{1} \frac{d^{4} \varphi}{d \rho^{4}}-\beta_{1} \frac{d^{2} \varphi}{d \rho^{2}} C_{1} C_{2} \varphi
\end{gathered}
$$

onde

$$
\begin{gathered}
a_{\alpha \beta}=\left(E_{\alpha \beta}-B_{\alpha \beta} \omega_{\alpha} \omega_{\beta}\right)\left(C_{1} \omega_{1}^{2}+C_{2} \omega_{2}^{2}\right) \\
f_{\alpha}=\left(E_{\alpha 3} \omega_{2}^{2}+B_{\alpha 3} \omega_{1}^{2}\right) \omega_{\alpha} \\
\alpha_{1}=D_{11} D_{66} \omega_{1}^{4}+\left(D_{11} D_{22}-D_{11}^{2} \nu_{y x}^{2}-2 D_{11} D_{66} \nu_{y x}\right) \omega_{1}^{2} \omega_{2}^{2}+D_{22} D_{66} \omega_{2}^{4} \\
\beta_{1}=\left(C_{2} D_{11}+C_{1} D_{66}\right) \omega_{1}^{2}+\left(C_{2} D_{66}+C_{1} D_{22}\right) \omega_{2}^{2} \\
\left.\tilde{P}_{\alpha \beta}(\rho)=\left[D_{\beta \gamma}\left(a_{\alpha \beta} \omega_{\gamma}+a_{\alpha \beta} \omega_{\beta}\right) n_{\gamma}+C_{\beta \gamma} d_{\alpha} n_{\gamma}\right] \frac{d^{5} \varphi}{d \rho^{5}}-\left[2 D_{\beta \gamma} C_{1} C_{2} \omega_{\alpha} \omega_{\beta} \omega_{\gamma}\right) n_{\gamma}+C_{1} C_{2} C_{\beta \gamma} \omega_{\alpha} n_{\gamma}\right] \frac{d^{3} \varphi}{d \rho^{3}} \\
\tilde{P}_{3 \alpha}(\rho)=\left[D_{\alpha \gamma}\left(f_{\alpha} \omega_{\gamma}+f_{\gamma} \omega_{\alpha}\right)+C_{\alpha \gamma} g\right] n_{\gamma} \frac{d^{4} \varphi}{d \rho^{4}}-\left[2 D_{\alpha \gamma} \omega_{\alpha} \omega_{\gamma}+C_{\alpha \gamma}\right] n_{\gamma} C_{1} C_{2} \frac{d^{2} \varphi}{d \rho^{2}}
\end{gathered}
$$




$$
\begin{gathered}
\tilde{P}_{\alpha 3}(\rho)=C_{\gamma}\left(a_{\alpha \gamma}-f_{\alpha} \omega_{\gamma}\right) n_{\gamma} \frac{d^{4} \varphi}{d \rho^{4}} \\
\tilde{P}_{33}(\rho)=C_{\gamma}\left(f_{\gamma}-\beta_{1} \omega_{\gamma}\right) n_{\gamma} \frac{d^{3} \varphi}{d \rho^{3}}+\alpha_{1} C_{\gamma} \omega_{\gamma} n_{\gamma} \frac{d^{5} \varphi}{d \rho^{5}}
\end{gathered}
$$

sendo que índices repetidos em $\alpha$ e $\beta$ não representam somatório:

$$
d_{\alpha}=a_{\alpha \zeta} \omega_{\zeta} \quad g=f_{\zeta} \omega_{\zeta}
$$

Os kernels $\tilde{U}$ são desenvolvidos no Apêndice H.

\subsubsection{Cálculo da solução fundamental}

1. Cálculo de $\varphi(\rho)$ e suas derivadas

Para encontrar os deslocamentos generelizados e os esforços generalizados, é necessário calcular a função $\varphi(\rho)$ e suas derivadas. O cálculo será simplificado se forem introduzidas as funções $A_{0}$ e $A_{1}$ dadas por:

$$
\begin{aligned}
& A_{0}(p \rho)=\exp (p \rho) \int_{\rho}^{\infty} \frac{\exp (-p \sigma)}{\sigma} d \sigma-\exp (-p \rho) \int_{\rho}^{-\infty} \frac{\exp (-p \sigma)}{\sigma} d \sigma \\
& A_{1}(p \rho)=\exp (p \rho) \int_{\rho}^{\infty} \frac{\exp (-p \sigma)}{\sigma} d \sigma+\exp (-p \rho) \int_{\rho}^{-\infty} \frac{\exp (-p \sigma)}{\sigma} d \sigma
\end{aligned}
$$

que, derivando com respeito a variável $\rho$, tem-se:

$$
\frac{d A_{0}(p \rho)}{d \rho}=p A_{1}(p \rho)-\frac{2}{p} \quad \frac{d A_{1}(p \rho)}{d \rho}=p A_{1}(p \rho) .
$$

Então, tem-se as expressões de $\varphi(\rho)$ e suas derivadas: 


$$
\begin{aligned}
\varphi(\rho) & =\frac{1}{8 \pi^{2} p^{4} a^{2}}\left(p^{2} \rho^{2} \log |\rho|+2 \log |\rho|+3+A_{0}(p \rho)\right) \\
\frac{d \varphi}{d \rho} & =\frac{1}{8 \pi^{2} p^{4} a^{2}}\left(2 p^{2} \rho \log |\rho|+p^{2} \rho+p A_{1}(p \rho)\right) \\
\frac{d^{2} \varphi}{d \rho^{2}} & =\frac{1}{8 \pi^{2} p^{4} a^{2}}\left(2 p^{2} \rho \log |\rho|+3 p^{2}+p^{2} A_{0}(p \rho)\right) \\
\frac{d^{3} \varphi}{d \rho^{3}} & =\frac{1}{8 \pi^{2} p^{4} a^{2}} p^{3} A_{1}(p \rho) \\
\frac{d^{4} \varphi}{d \rho^{4}} & =\frac{1}{8 \pi^{2} p^{4} a^{2}} p^{4} A_{0}(p \rho) \\
\frac{d^{5} \varphi}{d \rho^{5}} & =\frac{1}{8 \pi^{2} p^{4} a^{2}}\left(p^{5} A_{1}(p \rho)-\frac{2 p^{4}}{\rho}\right) \\
\frac{d^{6} \varphi}{d \rho^{6}} & =\frac{1}{8 \pi^{2} p^{4} a^{2}}\left(p^{6} A_{0}(p \rho)+\frac{2 p^{4}}{\rho^{2}}\right)
\end{aligned}
$$

2. Fórmulas para $A_{0}(p \rho)$ e $A_{1}(p \rho)$

As funções $A_{0}(p \rho)$ e $A_{1}(p \rho)$ podem ser expressas em termos das integrais exponenciais $E_{i}(p \rho)$ e $E_{1}(p \rho)$.

- $\operatorname{para} \rho>0$

$$
\begin{aligned}
& A_{0}(p \rho)=\exp (p \rho) E_{1}(p \rho)-\exp (-p \rho) E_{i}(p \rho) \\
& A_{1}(p \rho)=\exp (p \rho) E_{1}(p \rho)+\exp (-p \rho) E_{i}(p \rho)
\end{aligned}
$$

- $\operatorname{para} \rho<0$

$$
\begin{aligned}
& A_{0}(p \rho)=-\exp (-|p \rho|) E_{1}(|p \rho|)+\exp (|p \rho|) E_{1}(|p \rho|) \\
& A_{1}(p \rho)=-\exp (-|p \rho|) E_{1}(|p \rho|)-\exp (|p \rho|) E_{1}(|p \rho|)
\end{aligned}
$$

As fórmulas aproximadas de $E_{1}(p \rho)$ e $E_{i}(p \rho)$ podem ser encontradas em Abramowitz e Stegun (1965). 


\subsection{Tratamento numérico da solução fundamental}

A solução fundamental para cálculo de deslocamentos e esforços generalizados de superfície são dadas pelas equações (8.44) e (8.45), respectivamente. Os kernels para solução fundamental de deslocamentos são dados pelas equações (8.46) à (8.48) e os kernels da solução fundamental de forças de superfície são dadas pelas equações (8.50) à (8.53). Afim de realizar a integração das equações (8.44) e (8.45), primeiro determina-se o valor de $\theta_{o}$ que tem $\rho=0$. O valor de $\theta_{o}$ é determinado usando a seguinte expressão:

$$
\theta_{0}=\tan ^{-1}\left(-\frac{x-\xi}{y-\eta}\right)
$$

O intervalo de integração é dado por:

$$
\left[\theta_{0}, \theta_{0}+2 \pi\right]
$$

\subsubsection{Solução fundamental $U_{i j}^{*}$}

Como pode ser visto da equação (8.44), kernels dados pelas equações (8.46), (8.47) e (8.48) apresentam singularidades fracas que são tratadas usando quadratura de Gauss e transformada de Telles (ver Apêndice E).

As Figuras 8.1 à 8.9 mostram o comportamento dos kernels $U_{i j}$, considerando como ponto fonte $\xi=0,5$ e $\eta=0$ e ponto campo $x=0$ e $y=0,0094$. As propriedades dos materiais são: $E_{x}=20,0485 G P a, E_{y}=6,0039 G P a, G_{x y}=0,5 G P a, G_{x z}=0,2 G P a$, $\nu_{x y}=0,0417$ e $h=0,01 m$, e componentes do vetor normal são: $n_{x}=-1$ e $n_{y}=0$. 


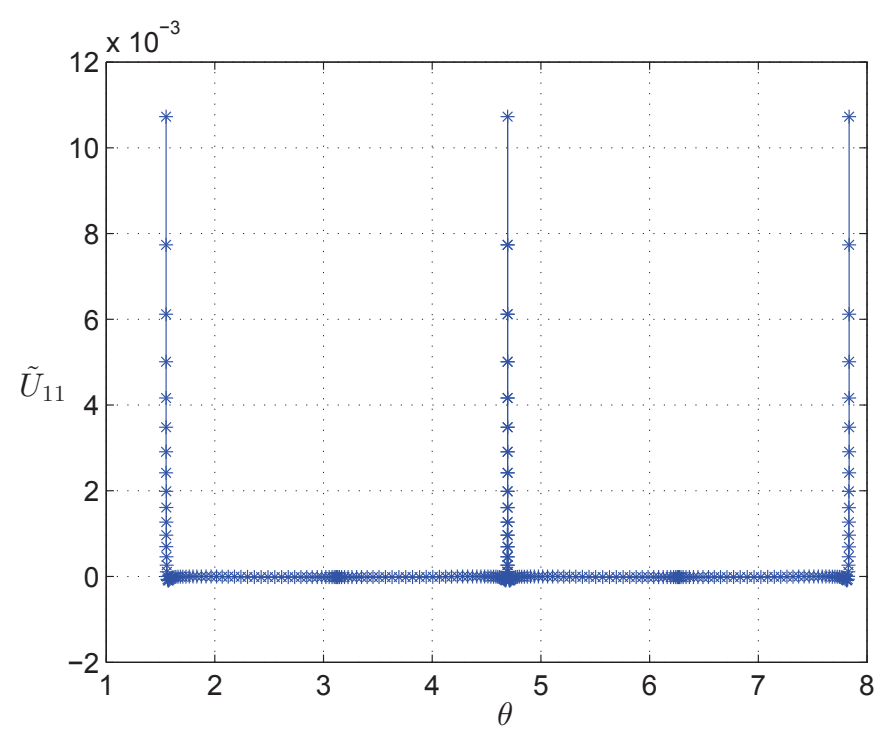

Figura 8.1: Kernel $\tilde{U}_{11}$.

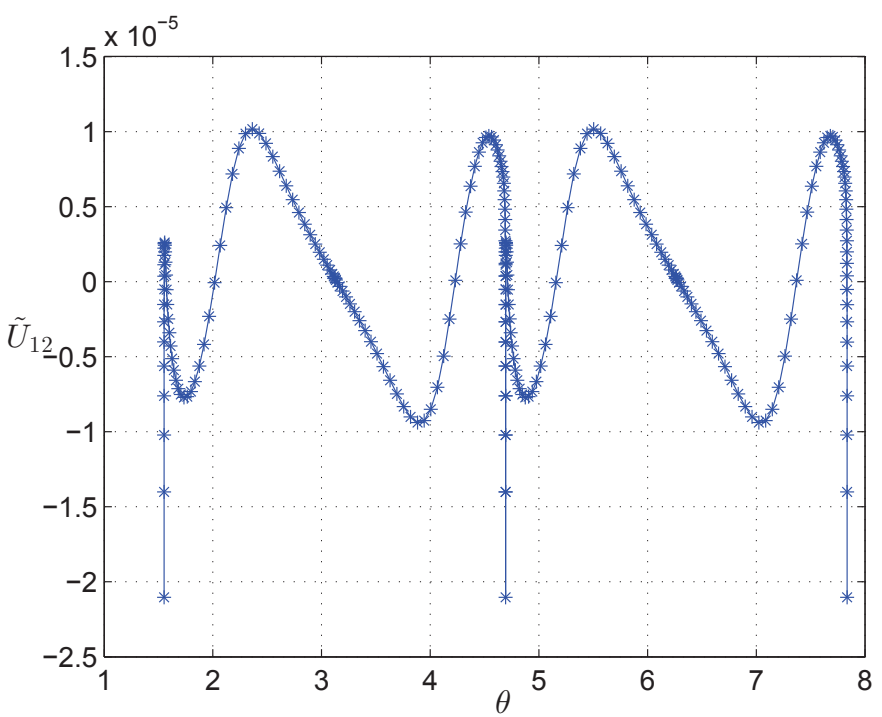

Figura 8.2: Kernel $\tilde{U}_{12}$. 


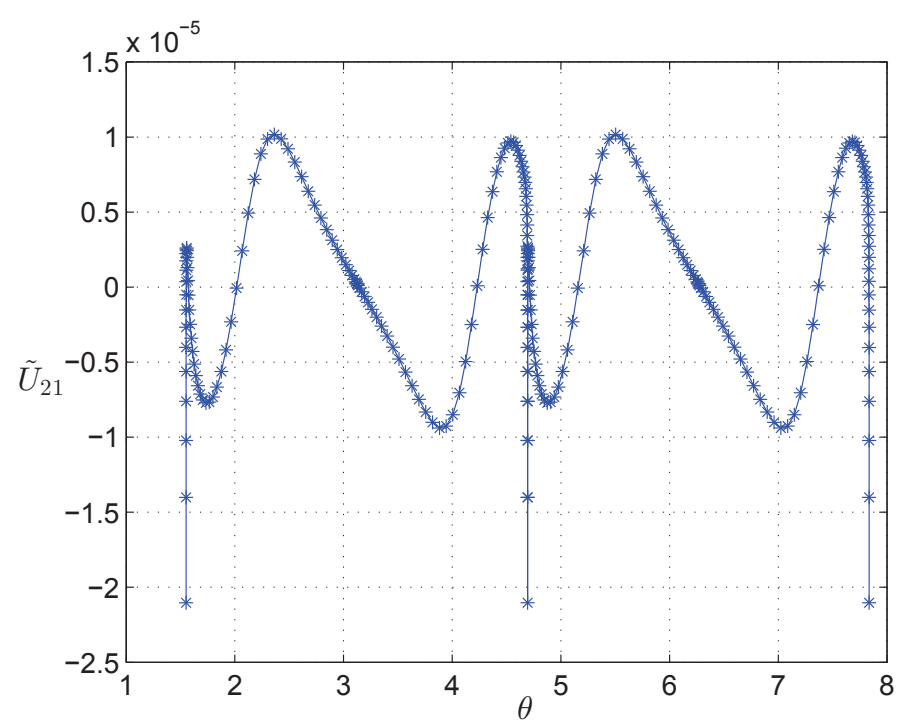

Figura 8.3: Kernel $\tilde{U}_{21}$.

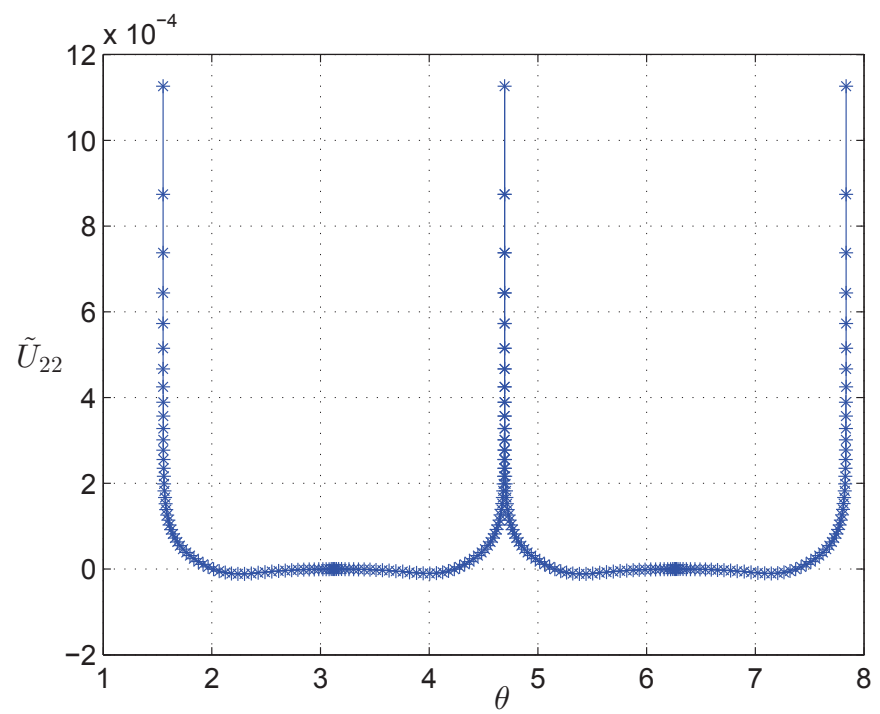

Figura 8.4: Kernel $\tilde{U}_{22}$. 


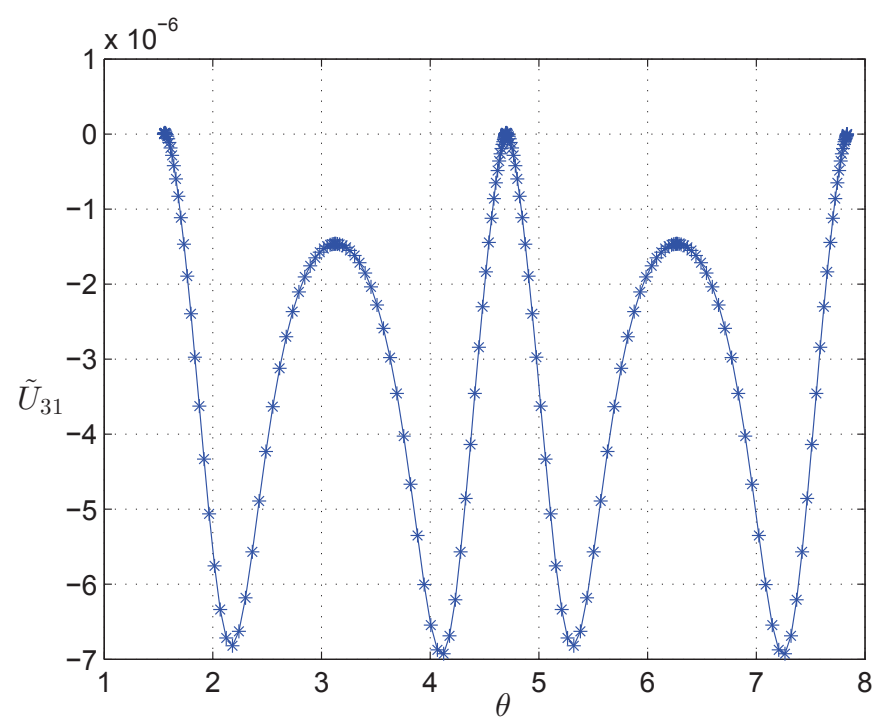

Figura 8.5: Kernel $\tilde{U}_{31}$.

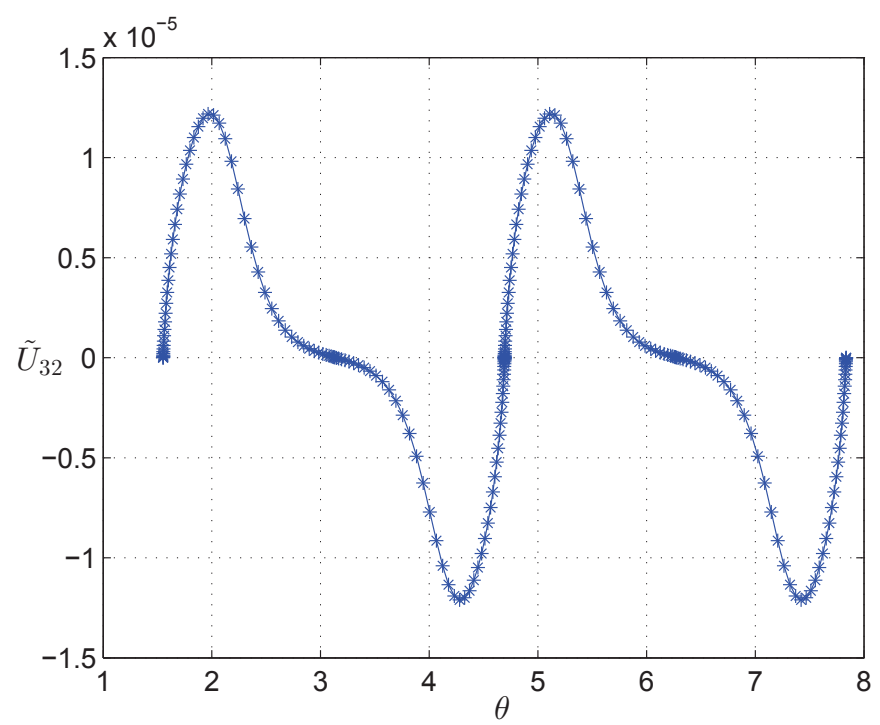

Figura 8.6: Kernel $\tilde{U}_{32}$. 


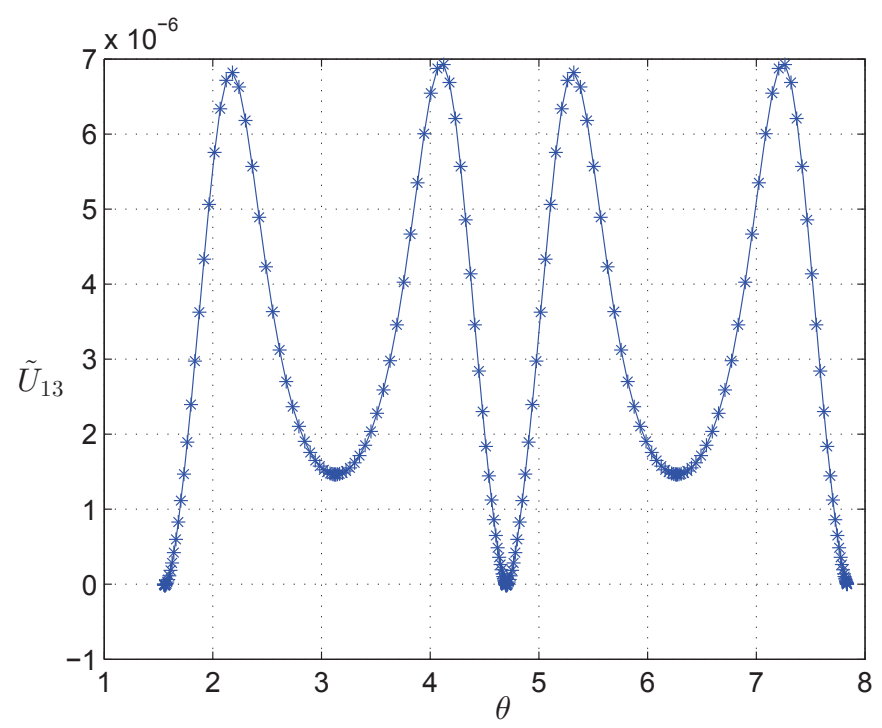

Figura 8.7: Kernel $\tilde{U}_{13}$.

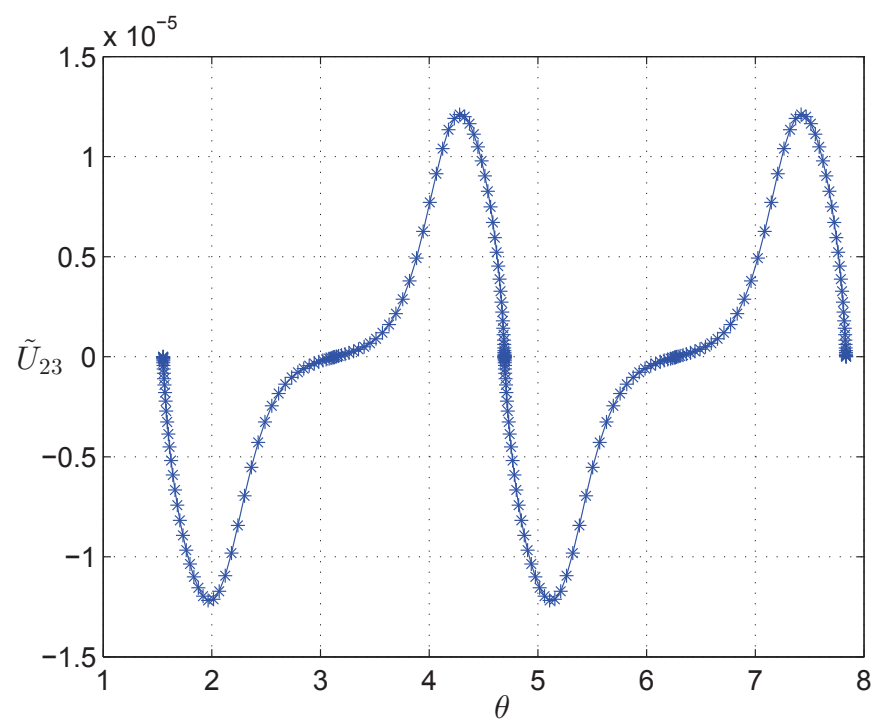

Figura 8.8: Kernel $\tilde{U}_{23}$. 


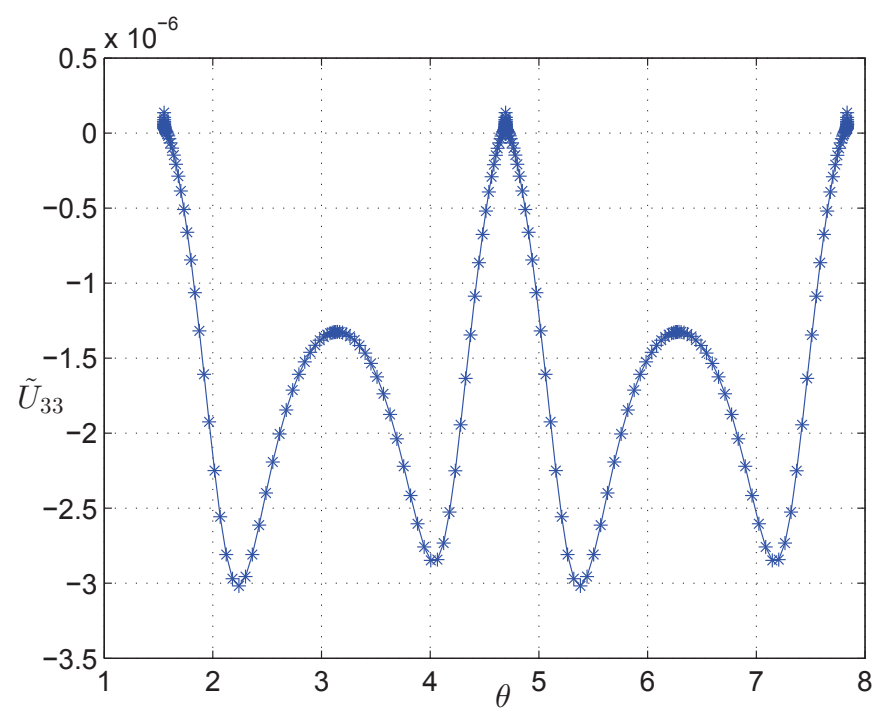

Figura 8.9: Kernel $\tilde{U}_{33}$.

Como pode ser visto nas Figuras 8.1 à 8.9, todos os kernels são simétricos em relação ao ângulo $\left(\theta=\theta_{0}+\pi\right)$ de integração. Devido esta simétria, somente metade do intervalo precisa ser integrado, isto é, a integração é realizada no intervalo $\left[\theta_{0}, \theta_{0}+\pi\right]$ e multiplicado por dois. Estes kernels são, no máximo, fracamente singular devido a presença da derivada $d^{4} \varphi(\rho) / d \rho^{4}$. Assim, a transformada de Telles é suficiente para obter uma integração exata. Dessa forma, a quadratura de Gauss com a transformada de Telles foi empregada para calcular a equação (8.44). 


\subsubsection{Solução fundamental $P_{i j}^{*}$}

Como pode ser visto na equação (8.45), os kernels dados pelas equações (8.50) e (8.53) apresentam singularidade forte. As Figuras 8.10 à 8.18 mostram o comportamento dos kernels $P_{i j}^{*}$, considerando o mesmo ponto fonte e campo e as mesmas propriedades do material dadas na seção anterior.

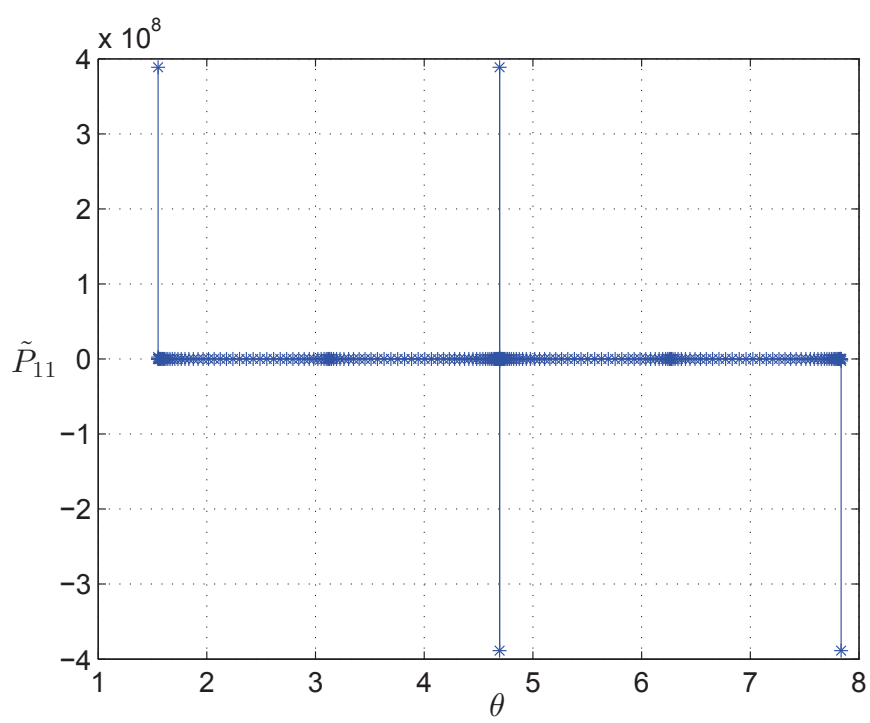

Figura 8.10: Kernel $\tilde{P}_{11}$.

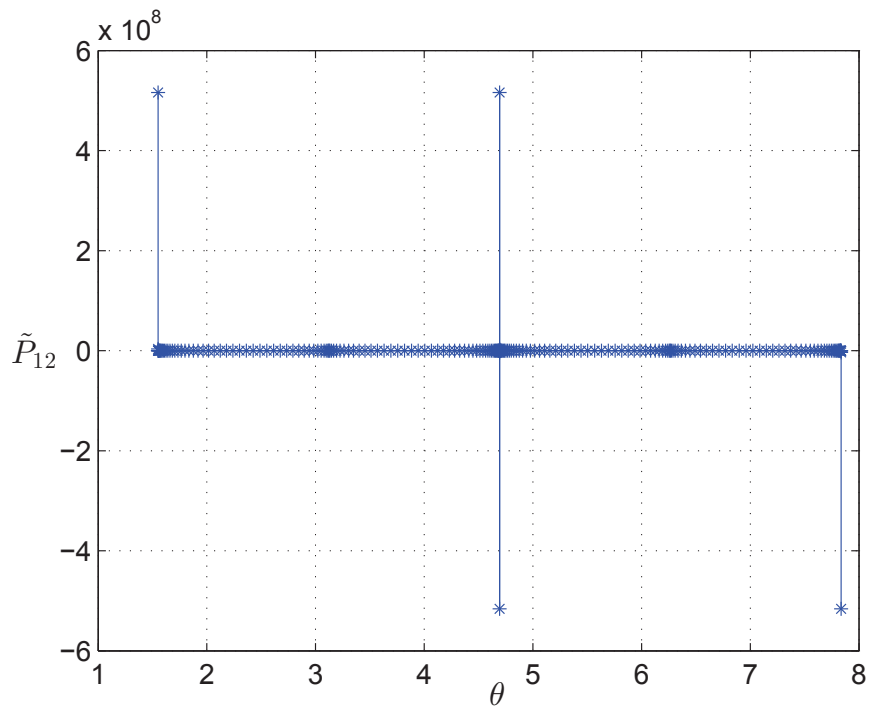

Figura 8.11: Kernel $\tilde{P}_{12}$. 


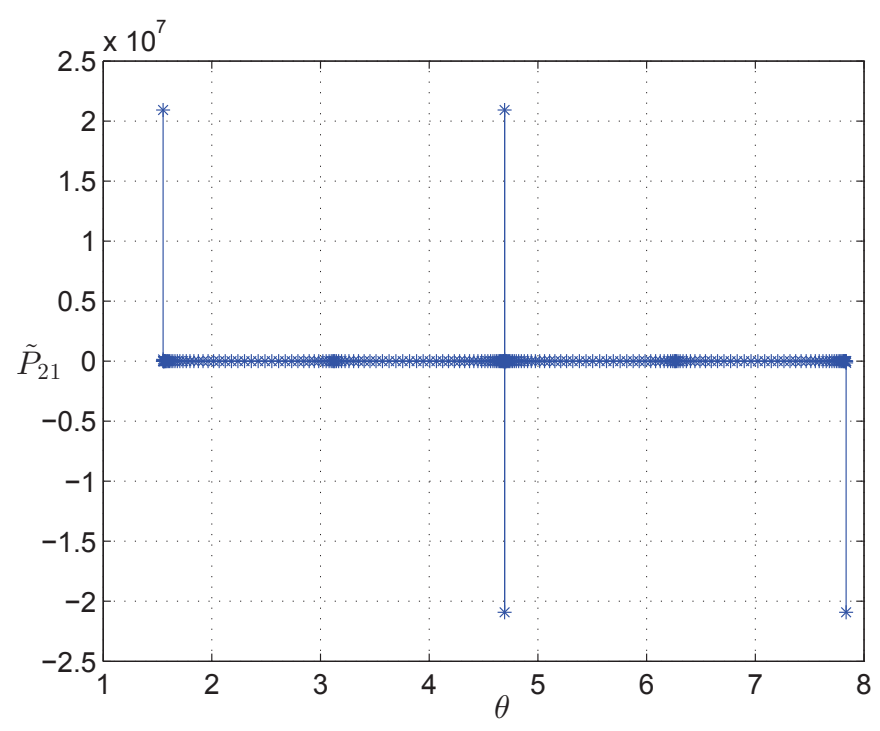

Figura 8.12: Kernel $\tilde{P}_{21}$.

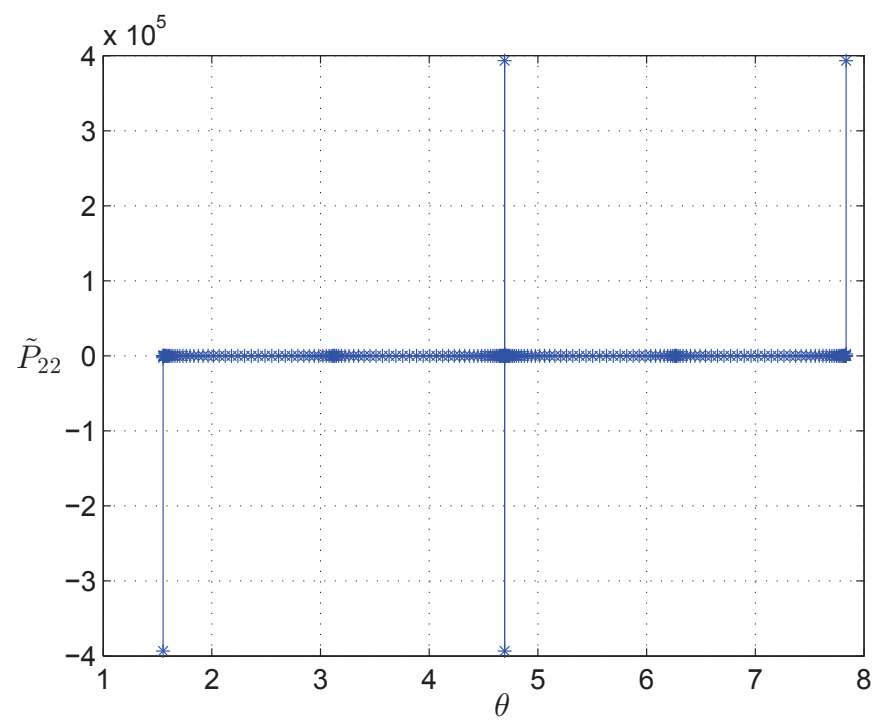

Figura 8.13: Kernel $\tilde{P}_{22}$. 


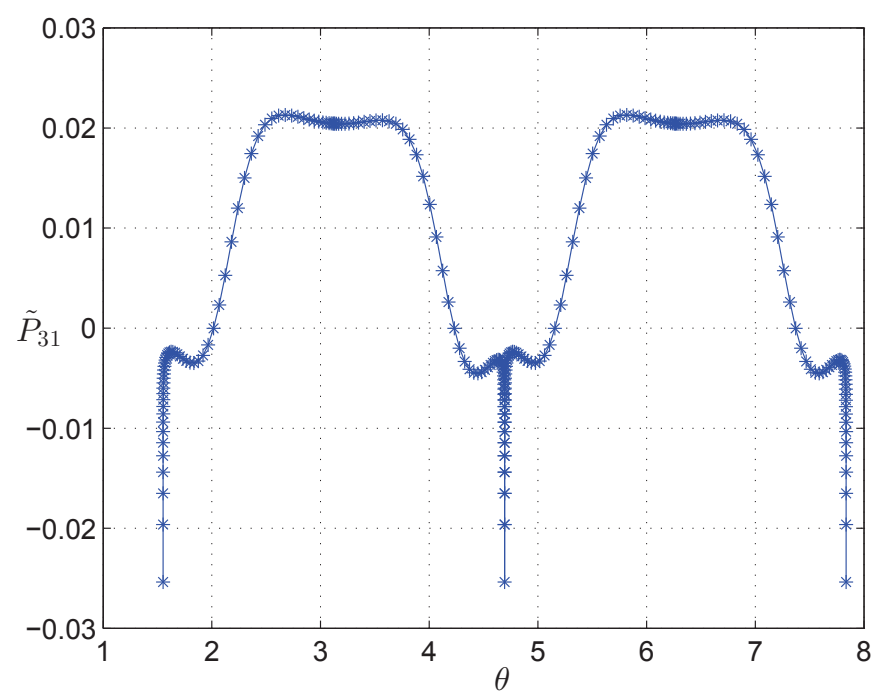

Figura 8.14: Kernel $\tilde{P}_{31}$.

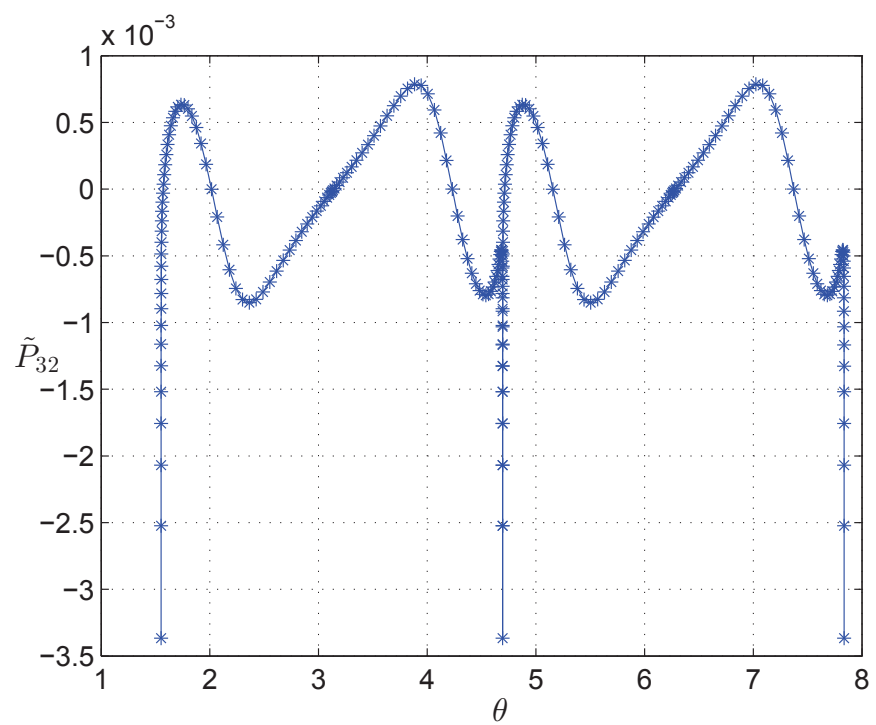

Figura 8.15: Kernel $\tilde{P}_{32}$. 


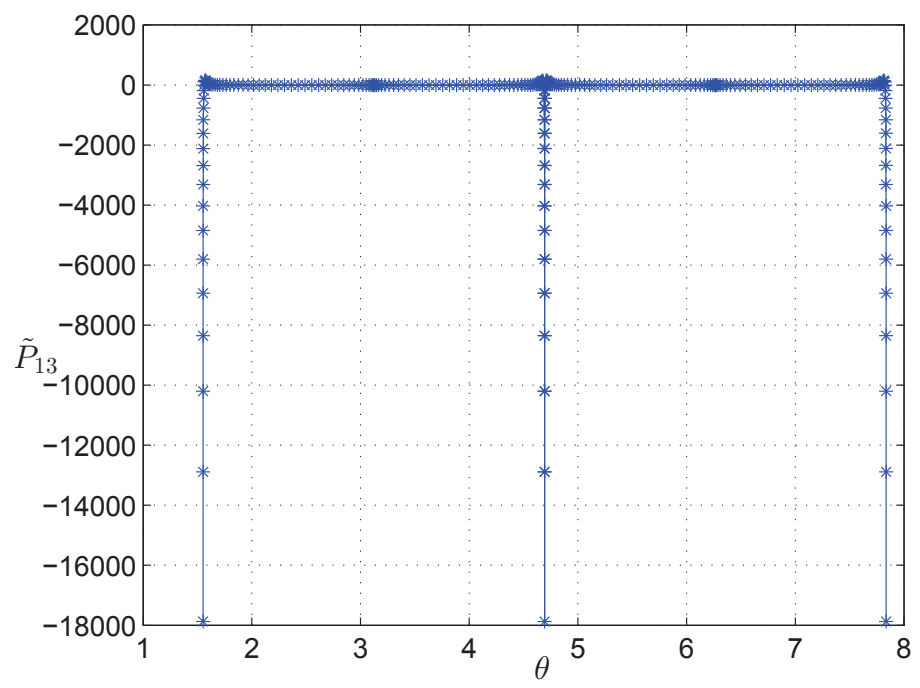

Figura 8.16: Kernel $\tilde{P}_{13}$.

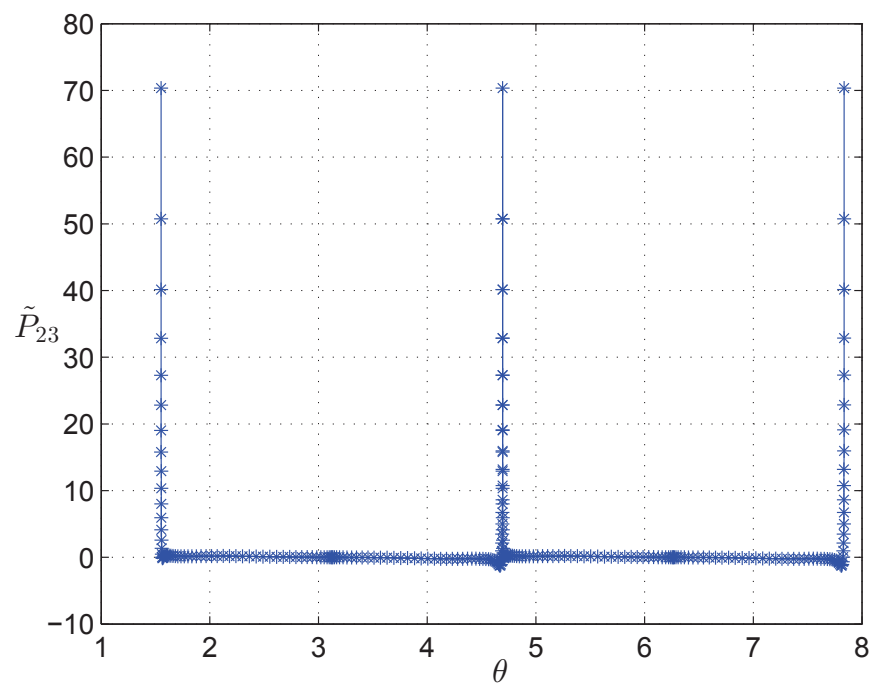

Figura 8.17: Kernel $\tilde{P}_{23}$. 


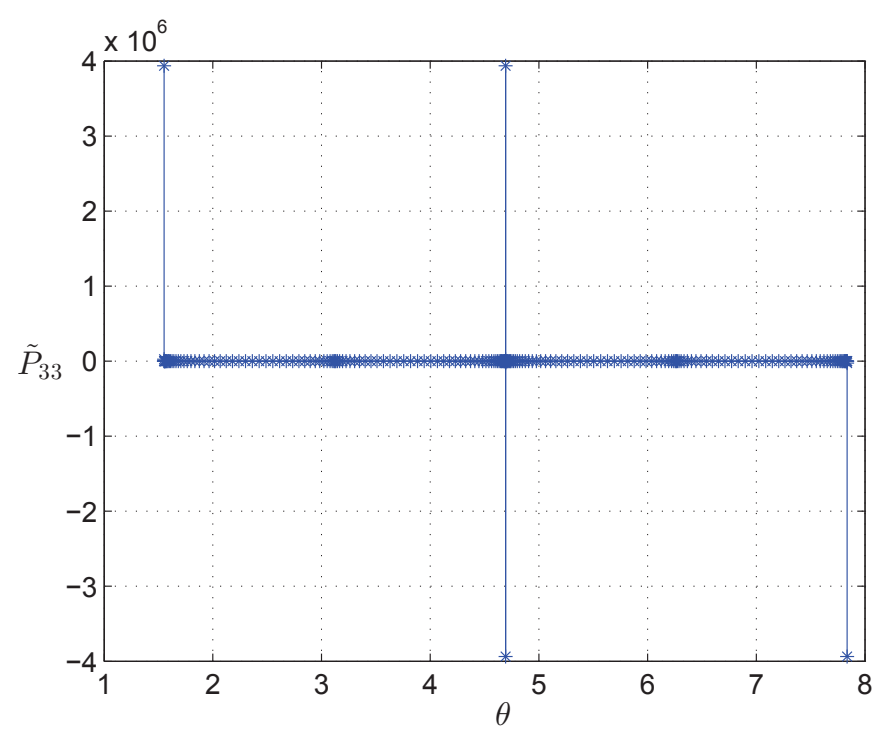

Figura 8.18: Kernel $\tilde{P}_{33}$.

As singularidades fortes presentes nos kernels dados pelas equações (8.51) à (8.53) podem ser tratadas usando integração simétrica, quadratura de Gauss e a transformada de Telles.

\subsection{Equações integrais de contorno}

A equação integral pode ser obtida considerando a representação integral da equação governante (4.45) e (4.46), ou seja:

$$
\int_{\Omega}\left[\left(M_{\alpha \beta, \beta}-Q_{\alpha}\right) U_{\alpha}^{*}+\left(Q_{\alpha, \alpha}+q\right) U_{3}^{*}\right] d \Omega=0
$$

onde $U_{i}^{*}(i=\alpha, 3)$ são as funções peso. Integrando por partes (aplicando a segunda identidade de Green) e usando as relações de reciprocidade, equação (8.15), obtém-se:

$$
U_{j}(\zeta)+\int_{\Gamma} P_{i j}^{*}(\zeta, x) U_{j}(x) d \Gamma=\int_{\Gamma} U_{i j}^{*}(\zeta, x) P_{j}(x) d \Gamma+\int_{\Omega} q(x) U_{i 3}^{*}(\zeta, x) d \Omega
$$

Considerando o ponto $\zeta$ no contorno $\Gamma$, a equação (8.64) pode ser escrita como:

$$
c_{i j}(\zeta) U_{j}(\zeta)+f_{\Gamma} P_{i j}^{*}(\zeta, x) U_{j}(x) d \Gamma=\int_{\Gamma} U_{i j}^{*}(\zeta, x) P_{j}(x) d \Gamma+\int_{\Omega} g(x) U_{i 3}^{*}(\zeta, x) d \Omega
$$


onde $f$ representa a integral no sentido do valor principal de Cauchy, $\zeta, x \in \Gamma$ são os pontos fonte e os pontos campo, respectivamente. O valor de $c_{i j}(x)$ é igual a $\delta_{i j} / 2$ quando $x$ está localizado num contorno suave. A equação (8.65) representa três equações integrais, duas $(i=\alpha=1,2)$ para rotações e uma $(i=3)$ para o deslocamento transversal. A última integral do lado direito na equação (8.65) é uma integral de domínio, que é transformada em uma integral de contorno pelo método da integração radial (RIM).

\subsubsection{Derivadas da solução fundamental $U_{i j}^{*}$ e $P_{i j}^{*}$}

Para o cálculo dos deslocamentos e forças de superfície nota-se as derivadas de $\rho$ variam de $d \varphi(\rho) / d \rho$ à $d^{4} \varphi(\rho) / d \rho^{4}$ para deslocamentos generalizados e de $d^{2} \varphi(\rho) / d \rho^{2}$ à $d^{5} \varphi(\rho) / d \rho^{5}$ para os esforços generalizados, ou seja, os kernels apresentam singularidades fraca $(\log (\rho))$ e forte $(1 / \rho)$ que podem ser tratadas usando pontos de Gauss e a transformada de Telles, desde que a integração seja feita de maneira simétrica em relação ao ponto de integração. Para o cálculo dos momentos usamos as relações para os esforços generalizados dados pelas equações (8.12) e (8.13). Dessa forma foi inserido nas equações (8.12) e (8.13) um termo $(\delta)$ para representar o ponto fonte da seguinte forma:

$$
\begin{gathered}
M_{\delta \alpha \beta}=D_{\alpha \beta}\left(\psi_{\delta \alpha, \beta}+\psi_{\delta \beta, \alpha}\right)+C_{\alpha \beta} \psi_{\delta \gamma, \gamma} \\
Q_{\delta \alpha}=C_{\alpha}\left(W_{\delta 3, \alpha}+\psi_{\delta \alpha}\right)
\end{gathered}
$$

onde $\psi$ representa as rotações com índices 1 e 2, e $W$ representa o delocamento na direção transversal com índice 3. Para o cálculo dos momentos os kernels das soluções fundamentais dadas pelas equações (8.44) e (8.45) aumentam suas singularidades em um grau. Dessa forma as equações (8.46) à (8.48) tomam a seguinte forma:

$$
\frac{d \tilde{U}_{\alpha \beta}(\rho)}{d \rho}=a_{\alpha \beta} \frac{d^{5} \varphi}{d \rho^{5}}-C_{1} C_{2} \omega_{\alpha} \omega_{\beta} \frac{d^{3} \varphi}{d \rho^{3}}
$$




$$
\begin{gathered}
\frac{d \tilde{U}_{3 \alpha}(\rho)}{d \rho}=-\frac{\tilde{U}_{\alpha 3}(\rho)}{d \rho}=f_{\alpha} \frac{d^{4} \varphi}{d \rho^{4}}-C_{1} C_{2} \omega_{\alpha} \frac{d \varphi^{2}}{d \rho^{2}} \\
\frac{d \tilde{U}_{33}(\rho)}{d \rho}=\alpha_{1} \frac{d^{4} \varphi}{d \rho^{4}}-\beta_{1} \frac{d^{2} \varphi}{d \rho^{2}} C_{1} C_{2} \varphi
\end{gathered}
$$

As equações (8.50) à (8.53) são derivadas em relação ao ponto fonte e dessa forma sao escritas como:

$$
\left.\frac{d \tilde{P}_{\alpha \beta}(\rho)}{d \rho}=\left[D_{\beta \gamma}\left(a_{\alpha \beta} \omega_{\gamma}+a_{\alpha \beta} \omega_{\beta}\right) n_{\gamma}+C_{\beta \gamma} d_{\alpha} n_{\gamma}\right] \frac{d^{6} \varphi}{d \rho^{6}}-\left[2 D_{\beta \gamma} C_{1} C_{2} \omega_{\alpha} \omega_{\beta} \omega_{\gamma}\right) n_{\gamma}+C_{1} C_{2} C_{\beta \gamma} \omega_{\alpha} n_{\gamma}\right] \frac{d^{4} \varphi}{d \rho^{4}}
$$

$$
\begin{gathered}
\frac{d \tilde{P}_{3 \alpha}(\rho)}{d \rho}=\left[D_{\alpha \gamma}\left(f_{\alpha} \omega_{\gamma}+f_{\gamma} \omega_{\alpha}\right)+C_{\alpha \gamma} g\right] n_{\gamma} \frac{d^{5} \varphi}{d \rho^{5}}-\left[2 D_{\alpha \gamma} \omega_{\alpha} \omega_{\gamma}+C_{\alpha \gamma}\right] n_{\gamma} C_{1} C_{2} \frac{d^{3} \varphi}{d \rho^{3}} \\
\frac{d \tilde{P}_{\alpha 3}(\rho)}{d \rho}=C_{\gamma}\left(a_{\alpha \gamma}-f_{\alpha} \omega_{\gamma}\right) n_{\gamma} \frac{d^{5} \varphi}{d \rho^{5}} \\
\frac{d \tilde{P}_{33}(\rho)}{d \rho}=C_{\gamma}\left(f_{\gamma}-\beta_{1} \omega_{\gamma}\right) n_{\gamma} \frac{d^{4} \varphi}{d \rho^{4}}+\alpha_{1} C_{\gamma} \omega_{\gamma} n_{\gamma} \frac{d^{6} \varphi}{d \rho^{6}}
\end{gathered}
$$

Fazendo $\delta=1, \alpha=1$ e $\beta=1$ obtemos a seguinte expressão para as equações (8.66) e $(8.67)$ :

$$
\begin{gathered}
M_{111}=D_{11}\left(\psi_{11,1}+\psi_{11,1}\right)+C_{11} \psi_{11,1} \\
Q_{11}=C_{1}\left(W_{13,1}+\psi_{11}\right)
\end{gathered}
$$

Transformando as notações indiciais dadas pelas equações (8.75) e (8.76) em elementos diferenciais na direção de $x$ ou $y$, obtém-se: 


$$
\begin{gathered}
M_{111}=D_{11}\left(\frac{d \psi_{11}}{d x}+\frac{d \psi_{11}}{d x}\right)+C_{11}\left(\frac{d \psi_{11}}{d x}+\frac{d \psi_{12}}{d y}\right) \\
Q_{11}=C_{1}\left(\frac{d W_{13}}{d x}+\psi_{11}\right)
\end{gathered}
$$

Nas equações (8.77) e (8.78) os termos estão no domínio diferencial de $d x$ e $d y$, porém as derivadas dos kernels dados pelas equações (8.68) à (8.70) e (8.71) à (8.74) estão no domínio $\rho$. Através da transformada de Radon, usa-se da seguinte propriedade:

$$
\frac{\partial \psi}{\partial x_{\alpha}}=\omega_{\alpha} \frac{d \psi}{d \rho}
$$

onde $\omega_{1}=\cos (\theta)$ e $\omega_{2}=\sin (\theta)$. Os valores de $\psi$ e $W$ assumem os valores de $\tilde{U}$ ou $\tilde{P}$, logo temos as seguintes equações:

$$
\begin{gathered}
M_{111}=D_{11}\left(\frac{d U_{11}}{d \rho} \omega_{1}+\frac{d U_{11}}{d \rho} \omega_{1}\right)+C_{11}\left(\frac{d U_{11}}{d \rho} \omega_{1}+\frac{d U_{12}}{d \rho} \omega_{2}\right) \\
Q_{11}=C_{1}\left(\frac{d U_{13}}{d \rho} \omega_{1}+U_{11}\right)
\end{gathered}
$$

e

$$
\begin{gathered}
M_{111}=D_{11}\left(\frac{d P_{11}}{d \rho} \omega_{1}+\frac{d P_{11}}{d \rho} \omega_{1}\right)+C_{11}\left(\frac{d P_{11}}{d \rho} \omega_{1}+\frac{d P_{12}}{d \rho} \omega_{2}\right) \\
Q_{11}=C_{1}\left(\frac{d P_{13}}{d \rho} \omega_{1}+P_{11}\right)
\end{gathered}
$$

Para maiores detalhes ver o Apêndice G. 


\subsubsection{Tratamento de singularidades}

As singularidades presentes nas equações (8.68) à (8.70) dependem no máximo de $d^{5} d \varphi / d \rho^{5}$. A derivada $d^{5} \varphi / d \rho^{5}$ é fortemente singular, pois possui um termo da ordem de $1 / \rho$. Neste caso, a transformada de Telles, é possível tratar a singularidade, desde que os pontos de integração sejam colocados simetricamente. Por outro lado, nas equações (8.71) à (8.74) dependem no máximo de $d^{6} d \varphi / d \rho^{6}$. A derivada $d^{6} d \varphi / d \rho^{6}$ é hipersingular, pois apresenta um termo da ordem de $1 / \rho^{2}$. Neste caso somente com a quadratura de Gauss e a transformada de Telles é impossível tratar a singularidade. A quadratura proposta por Campos e Albuquerque (2013) foi usada para tratar tal tipo de singularidade (ver Apêndice F). Por exemplo, considere a equação (8.58):

$$
\frac{d^{6} \varphi}{d \rho^{6}}=\frac{1}{8 \pi^{2} p^{4} a^{2}}\left(p^{6} A_{o}(p \rho)+\frac{2 p^{4}}{\rho^{2}}\right)
$$

O primeiro termo do lado direito entre parênteses da equação (8.58) é tratado usando a quadratura de Gauss e a transformada de Telles com 6 pontos de integração e o segundo termo é multiplicado por zero. O segundo termo do lado direito entre parênteses da equação (8.58) é tratado usando a quadratura de Campos e Albuquerque (2013) com 6 pontos de integração e o primeiro termo é multiplicado por zero. As quadraturas foram implementadas da seguinte forma:

- Gauss e Telles:

$$
\frac{d^{6} \varphi}{d \rho^{6}}=\frac{1}{8 \pi^{2} p^{4} a^{2}}\left(p^{6} A_{o}(p \rho)+\frac{2 p^{4}}{\rho^{2}} \times 0\right)
$$

- Campos e Albuquerque:

$$
\frac{d^{6} \varphi}{d \rho^{6}}=\frac{1}{8 \pi^{2} p^{4} a^{2}}\left(p^{6} A_{o}(p \rho) \times 0+\frac{2 p^{4}}{\rho^{2}}\right)
$$

Em seguida os termos são somados da seguinte maneira: 


$$
\frac{d^{6} \varphi}{d \rho^{6}}=\frac{1}{8 \pi^{2} p^{4} a^{2}}\left(p^{6} A_{o}(p \rho)+\frac{2 p^{4}}{\rho^{2}}\right)
$$

\subsubsection{Validação dos resultados}

Afim de validar os resultados, foi usado o método das diferenças finitas (ver Apêndice C). Os kernels da solução fundamental $U_{i j}^{*}$ são validados com o método das diferenças finitas (MDF) e usando a quadratura de Gauss e transformada de Telles, pois apresentam singularidades no máximo de $1 / r$ e $\log r$. Os kernels da solução fundamental de $P_{i j}^{*}$ são validados com o método das diferenças finitas (MDF) e usando a quadratura de Campos e Albuquerque (2013), pois apresentam singularidades de no máximo $1 / r^{2}$. Os únicos termos das derivadas dos kernels da solução fundamental $P_{i j}^{*}$ que são usados a quadratura de Gauss e a transformada de Telles são $d P(1,3) / d x$ e $d P(2,3) / d x$, pois são mais fáceis de integrar que os demais termos. As equações de diferenças finitas utilizadas para validar a formulação são dadas por:

$$
P_{i j k}^{*}=\frac{d P_{i j}^{*}}{d x}=\lim _{\Delta x \longrightarrow 0} \frac{P(x+\Delta x)-P(x)}{\Delta x}
$$

As Tabelas 8.1, 8.2 e 8.3 mostra a comparação entre os métodos considerando como ponto fonte $\xi=0,5$ e $\eta=0$ e ponto campo $x=1$ e $y=0,0094$. As propriedades dos materiais são: $E_{x}=25 \mathrm{MPa}, E_{y}=50 \mathrm{MPa}, G_{x y}=10 \mathrm{MPa}, G_{x z}=20 \mathrm{MPa}, G_{y z}=30 \mathrm{MPa}$, $\nu_{x y}=0,25$ e $h=0,254 m$, e componentes do vetor normal são: $n_{x}=\sqrt{2} / 2$ e $n_{y}=\sqrt{2} / 2$. Os resultados apresentaram boa concordância entre o método dos elementos de contorno (MEC) e método das diferenças finitas (MDF), como mostrado nas Tabelas 8.1, 8.2 e 8.3. 
Tabela 8.1: Matriz $P_{i j k}(:,:, 1)$ obtida através do MEC e MDF.

\begin{tabular}{|c|c|c|c|}
\hline \multicolumn{2}{|c|}{ MEC $\times 10^{6}$} & \multicolumn{2}{c|}{ MDF $\times 10^{6}$} \\
\hline \hline 1,4020 & $-0,4604$ & 1,4061 & $-0,4625$ \\
\hline$-0,4604$ & $-0,6233$ & $-0,4625$ & $-0,6207$ \\
\hline 8,0092 & 2,3364 & 8,0188 & 2,3439 \\
\hline
\end{tabular}

Tabela 8.2: Matriz $P_{i j k}(:,:, 2)$ obtida através do MEC e MDF.

\begin{tabular}{|c|c|c|c|}
\hline \multicolumn{2}{|c|}{ MEC $\times 10^{6}$} & \multicolumn{2}{c|}{ MDF $\times 10^{6}$} \\
\hline \hline 0,1943 & $-0,2301$ & 0,1955 & $-0,2294$ \\
\hline$-0,2301$ & $-0,3366$ & $-0,2294$ & $-0,3351$ \\
\hline$-5,1090$ & $-5,3527$ & $-5,1264$ & $-5,3443$ \\
\hline
\end{tabular}

Tabela 8.3: Matriz $P_{i j k}(:,:, 3)$ obtida através do MEC e MDF.

\begin{tabular}{|c|c|c|c|}
\hline \multicolumn{2}{|c|}{ MEC $\times 10^{6}$} & \multicolumn{2}{c|}{ MDF $\times 10^{6}$} \\
\hline \hline$-0,3592$ & $-0,1856$ & $-0,3593$ & $-0,1858$ \\
\hline$-0,1856$ & $-0,2776$ & $-0,1858$ & $-0,2770$ \\
\hline$-3,6392$ & 3,5040 & $-3,6471$ & 3,5119 \\
\hline
\end{tabular}




\section{Capítulo 9}

\section{Resultados para placas ortotrópicas}

\subsection{Resultados}

Nesta seção, são obtidos resultados numéricos para deslocamentos e esforços (momento e tensão) em placas espessas ortotrópicas sujeitas a carregamentos distribuídos em seu domínio. Estes resultados são comparados com resultados presentes na literatura. Os valores obtidos para deslocamentos, momentos e tensões puderam ser comparados com resultados existentes na literatura (Sladek et al., 2006). Para tensões os resultados não puderam ser comparados diretamente, visto que, na literatura, não encontrou-se resultados equivalentes ao problema apresentado. Os resultados para tensões foram comparados com o método dos elementos finitos (MEF) utilizando-se do programa comercial ANSYS. Para todos os problemas seguintes foram estudados a influência do número de elementos no contorno. 


\subsubsection{Placa quadrada apoiada nos quatro lados sob carga unifor- memente distribuída}

Considere uma placa apoiada (Figura 9.1) carregada por uma carga $q=2,07 \times 10^{6}$ $\mathrm{N} / \mathrm{m}^{2}$ com sentido contrário a $x_{3}$. A placa é isotrópica e apresenta as seguintes propriedades e dimensões: $E_{y}=0,6895 \times 10^{10} \mathrm{~Pa}, E_{x}=2 \times E_{y}, G_{x y}=G_{x z}=G_{y z}=E_{y} / 2\left(1+\nu_{x y}\right) \mathrm{e}$ $\nu_{x y}=0,3$. Foram analisados diferentes valores de $h / a$. Os resultados numéricos obtidos pelo presente método implementado é equivalente ao problema proposto por Sladek et al. (2006) ${ }^{1}$ que foi analisado usando a formulação do método sem malha Petrov-Galerking (MLPG). Foram utilizados 6 pontos de integração usando a quadratura de Gauss (Abramowitz e Stegun, 1965) e transformada de Telles (1987) e para quadratura proposta por Campos e Albuquerque (2013) foram utilizados 6 pontos de integração para obter uma boa convergência nos resultados.

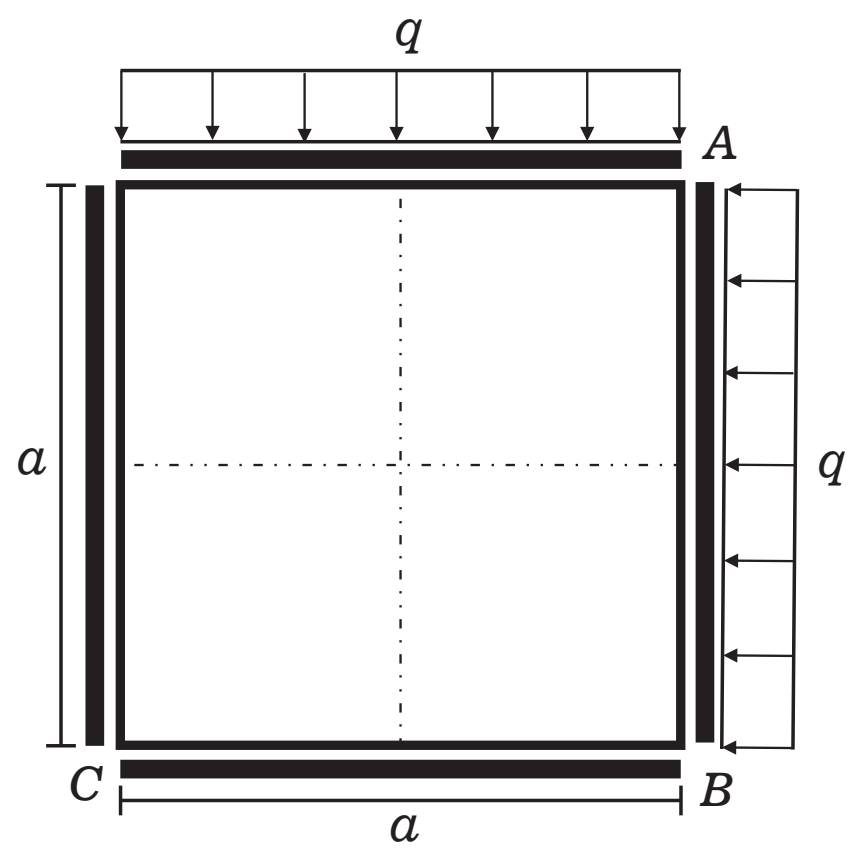

Figura 9.1: Placa quadrada apoiada nos quatro lados.

\footnotetext{
${ }^{1}$ Ao longo de todo texto será citado apenas Sladek ao invés de Sladek et. al., afim de minimizar largura das tabelas no texto.
} 
Para placa apoiada as seguintes condições de contorno são conhecidas ao longo dos lados da placa mostrada acima:

$$
u_{t}=0 \longleftrightarrow u_{3}=0 \longleftrightarrow u_{n} \neq 0 \longleftrightarrow M_{t} \neq 0 \longleftrightarrow M_{n}=0
$$

A Tabela 9.1 apresenta os resultados de deslocamento vertical e momento no centro da placa apoiada para as relações de espessura/lado: $h / a=0,05$ e $h / a=0,1$. Os resultados apresentam boa concordância com a literatura. Pode-se notar que as diferenças nos resultados diminuem com o aumento da espessura. Foram usados 48 elementos no contorno de mesmo comprimento (12 elementos por lado) e 1 nó interno (Figura 9.2).

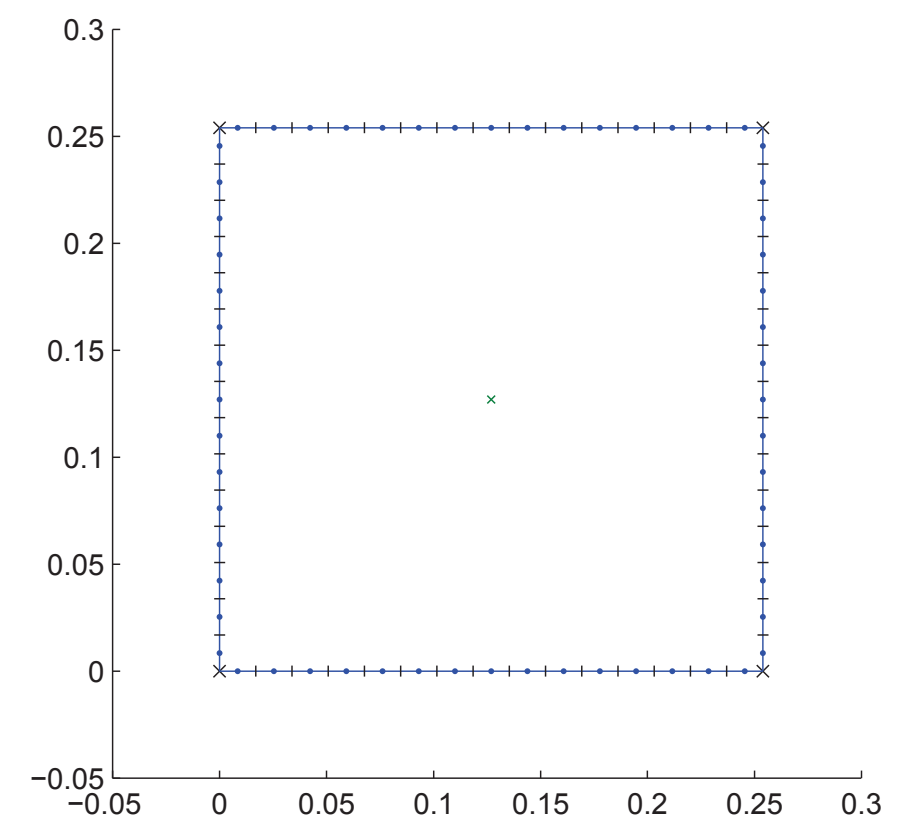

Figura 9.2: Discretização com 48 elementos no contorno e 1 nó interno. 
Tabela 9.1: Deslocamento transversal, momentos e tensões no centro da placa apoiada.

\begin{tabular}{|c|c|c|c|c|c|c|c|}
\hline \multirow{2}{*}{$h / a$} & \multicolumn{3}{|c|}{$w \times 10^{-3}(\mathrm{~m})$} & \multicolumn{3}{c|}{$M_{x x}(\mathrm{~N} . \mathrm{m})$} & $\sigma_{x x} \times 10^{8}\left(\mathrm{~N} / \mathrm{m}^{2}\right)$ \\
\cline { 2 - 8 } & MEC & Sladek (2006) & Dif. (\%) & MEC & Sladek (2006) & Dif. (\%) & MEC \\
\hline 0,05 & 23,51 & 23,42 & 0,38 & 9403,4 & 9333 & 0,75 & 3,6037 \\
\hline 0,1 & 3,17 & 3,17 & 0,01 & 9309,3 & 9540 & 2,40 & 0,9259 \\
\hline
\end{tabular}

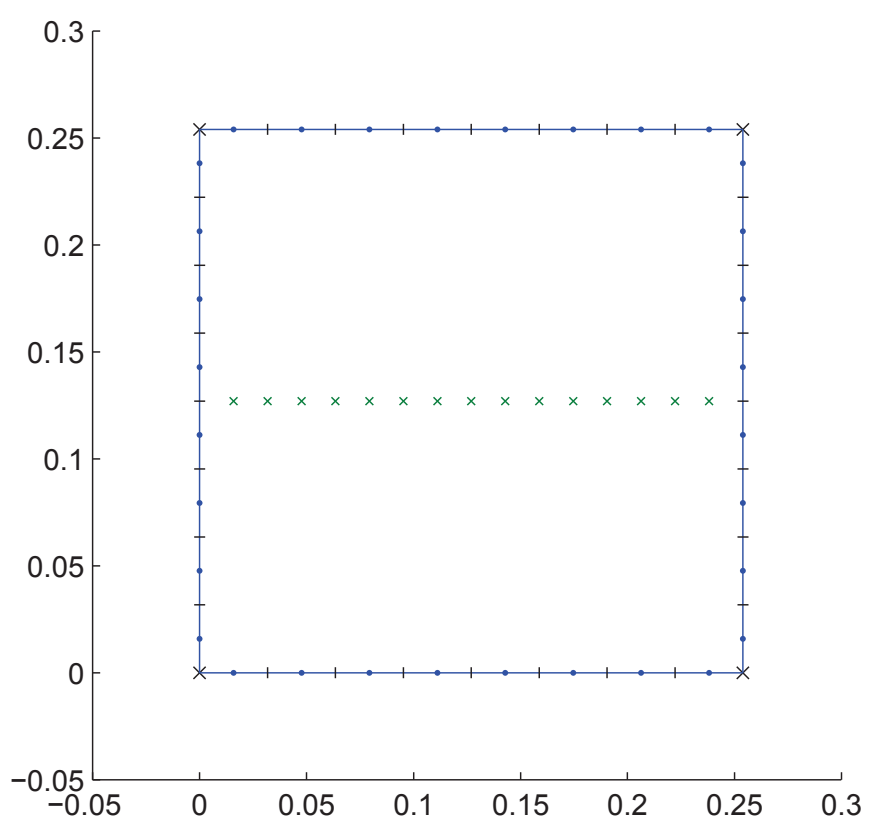

Figura 9.3: Discretização com 32 elementos no contorno e 15 nós internos.

A Figura 9.4 mostra a variação do deslocamento transversal $w$ ao longo da linha central da placa $(y=a / 2)$, considerando uma placa isotrópica e ortotrópica. Como em Sladek et al. (2006), os deslocamentos e momentos são normalizados, respectivamente, pelo valor do deslocamento transversal de uma placa isotrópica $w^{(i s o)}(a / 2)=28,29 \times 10^{-3}$ m e $m_{x x}^{(i s o)}(a / 2)=$ 7064 N.m. Pode ser observado que existe uma boa concordância entre ambos os resultados. Como pode-se ver na Figura 9.4, o deslocamento vertical da placa ortotrópica é reduzido em relação a placa isotrópica. Entretanto, é observado que o momento $\left(m_{x x}\right)$ na placa ortotrópica, apresenta aumento em relação a placa isotrópica (Figura 9.5). A Figura 9.3 
mostra a geometria gerada pelo código usando-se 8 elementos por lado e 15 pontos internos (onde deslocamentos e momentos foram calculados).

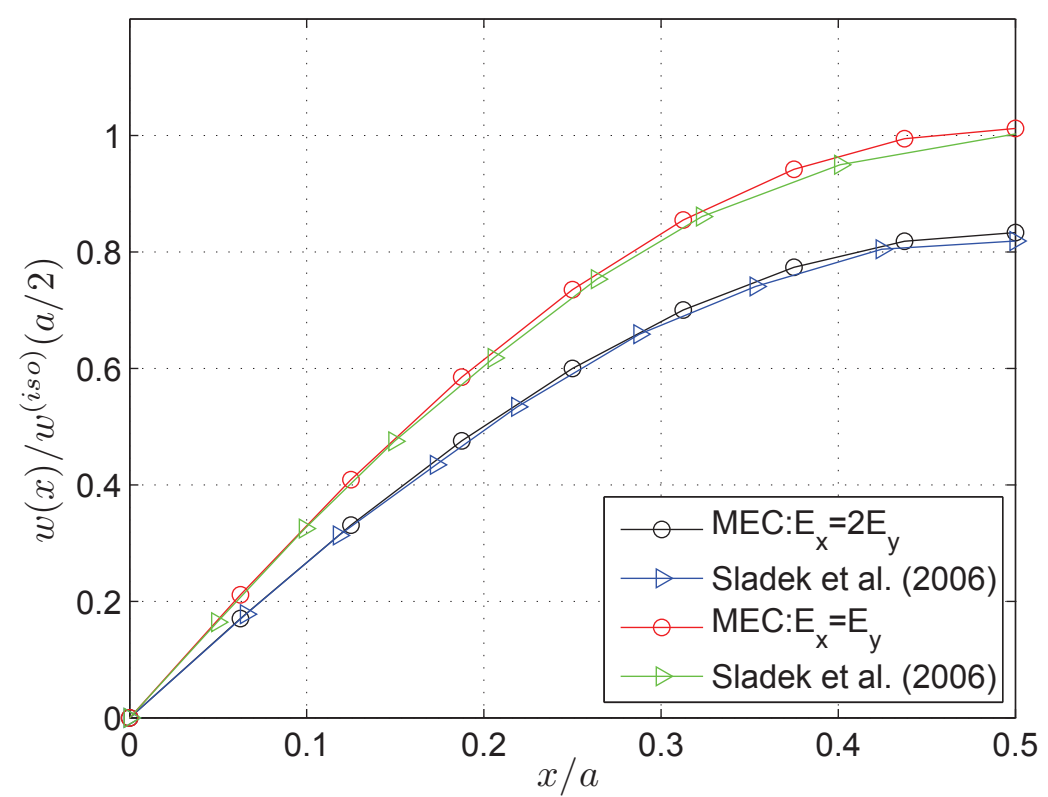

Figura 9.4: Variação do deslocamento transversal $w$ devido um carregamento estático uniformemente distribuído. 


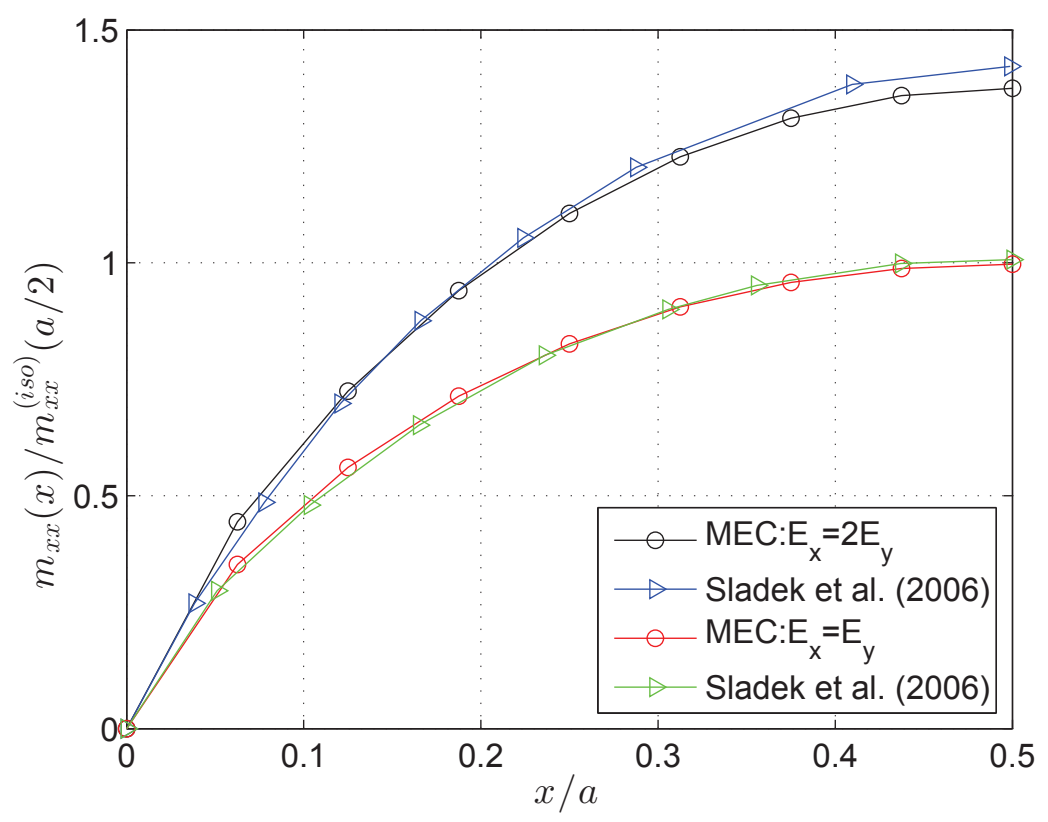

Figura 9.5: Variação do momento $m_{x x}$ devido um carregamento estático uniformemente distribuído.

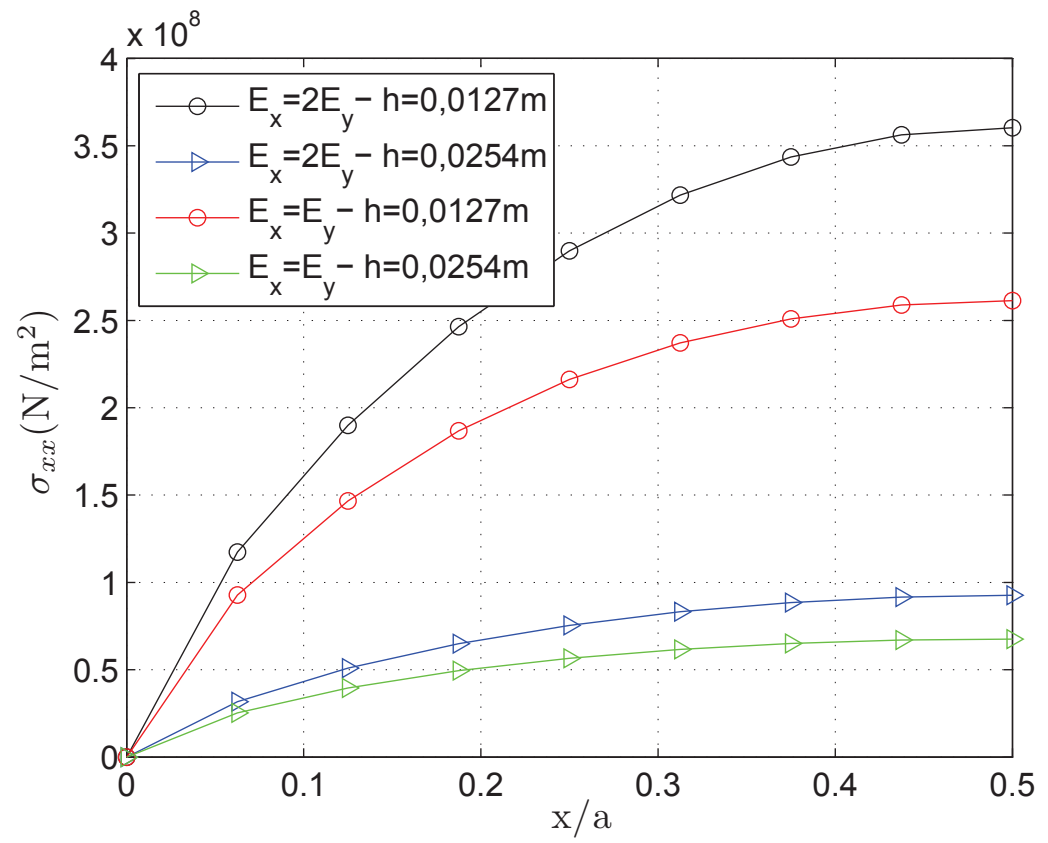

Figura 9.6: Variação da tensão $\sigma_{x x}$ devido um carregamento estático uniformemente distribuído. 
A Figura 9.6 mostra a variação da tensão $\left(\sigma_{x x}\right)$ ao longo da linha central da placa $(y=a / 2)$, considerando uma placa isotrópica e ortotrópica. Pode-se notar que as tensões apresentam valores superiores para placas ortotrópicas.

\subsubsection{Influência do número de elementos no contorno}

A placa foi discretizada usando-se 20, 40, 60, 80 e 100 elementos no contorno, respectivamente. Foi usado somente 1 ponto interno e foram obtidos resultados para deslocamento vertical e momento do nó central.

Tabela 9.2: Valores obtidos no centro da placa apoiada de espessura $h=0,0127 \mathrm{~m}$.

\begin{tabular}{|c|c|c|c|c|}
\hline \multirow{2}{*}{ MALHA } & \multicolumn{2}{|c|}{ Deslocamento } & \multicolumn{2}{c|}{ Momento } \\
\cline { 2 - 5 } & $w\left(\times 10^{-3} \mathrm{~m}\right)$ & Dif. (\%) & $m_{x x}$ (N.m) & Dif. (\%) \\
\hline 20 & 23,8 & 1,62 & 9558,4 & 2,41 \\
\hline 40 & 23,5 & 0,34 & 9411,2 & 0,83 \\
\hline 60 & 23,5 & 0,34 & 9403,6 & 0,75 \\
\hline 80 & 23,5 & 0,34 & 9406,9 & 0,79 \\
\hline 100 & 23,5 & 0,34 & 9411,5 & 0,84 \\
\hline 120 & 23,5 & 0,34 & 9415,8 & 0,88 \\
\hline Sladek $(2006)$ & \multicolumn{3}{|c|}{23,42} & \multicolumn{3}{c|}{9333} \\
\hline
\end{tabular}

Pode-se notar na Tabela 9.2 que as diferenças nos resultados diminuem a medida que aumenta o números de nós no contorno tanto para deslocamento quanto para momento. Nota-se também que as diferenças nos resultados apresentam-se pouco significativos para deslocamentos (em torno de 0,34\%), para momentos estabilizaram-se para a malha de 40 elementos (20 elementos por lado) e começa a divergir para malha de 80 elementos (20 elementos por lado). 
Para o cálculo dos momentos no contorno de uma placa apoiada foi considerada uma placa retangular robusta com as seguintes dimensões $a=2 \mathrm{~m}, b=0,2 \mathrm{~m}$ e espessuras iguais a $h=0,2 \mathrm{~m}$ (10\% do lado $a), h=0,3 \mathrm{~m}(15 \%$ do lado $a)$ e $h=0,4 \mathrm{~m}(20 \%$ do lado $a)$. Para tal condição tem-se os momentos tangencial e normal ao contorno da placa, onde $M_{t} \neq 0$ e $M_{n}=0$. O interesse deste problema é mostrar os momentos tangenciais no contorno da placa. A relação entre os momentos e a normal ao ponto fonte (ver equações (5.92) e (5.93)). Para tal problema analisamos um placa ortotrópica que apresenta as seguintes propriedades: $E_{y}=0,6895 \times 10^{10} \mathrm{~Pa}, E_{x}=2 \times E_{y}, G_{x y}=G_{x z}=G_{y z}=E_{y} / 2\left(1+\nu_{x y}\right)$ e $\nu_{x y}=0,3$. A placa apoiada é carregada por uma carga $q=1 \mathrm{~N} / \mathrm{m}^{2}$ com sentido contrário a $x_{3}$. Os resultados são mostrados na Tabela (9.3).

Tabela 9.3: Momento no lado BC da placa apoiada.

\begin{tabular}{|c|c|c|c|c|c|c|}
\hline \multirow{2}{*}{$x$} & \multicolumn{2}{|c|}{$h=0,2(\mathrm{~m})$} & \multicolumn{2}{c|}{$h=0,3(\mathrm{~m})$} & \multicolumn{2}{c|}{$h=0,4(\mathrm{~m})$} \\
\cline { 2 - 7 } & $p(\mathrm{~N} . \mathrm{m})$ & $C D C$ & $p(\mathrm{~N} . \mathrm{m})$ & $C D C$ & $p(\mathrm{~N} . \mathrm{m})$ & $C D C$ \\
\hline 0,1 & 0,2804 & 0,0739 & 0,1365 & 0,0741 & 0,1056 & 0,0741 \\
\hline 0,3 & 0,3447 & 0,1044 & 0,1699 & 0,1030 & 0,1281 & 0,1021 \\
\hline 0,5 & 0,2339 & 0,1009 & 0,1510 & 0,1010 & 0,1238 & 0,1007 \\
\hline 0,7 & 0,1641 & 0,1004 & 0,1324 & 0,1005 & 0,1178 & 0,1005 \\
\hline 0,9 & 0,1396 & 0,1002 & 0,1241 & 0,1003 & 0,1148 & 0,1004 \\
\hline 1,1 & 0,1401 & 0,1002 & 0,1244 & 0,1004 & 0,1149 & 0,1004 \\
\hline 1,3 & 0,1646 & 0,1004 & 0,1327 & 0,1005 & 0,1180 & 0,1005 \\
\hline 1,5 & 0,2342 & 0,1009 & 0,1512 & 0,1009 & 0,1239 & 0,1007 \\
\hline 1,7 & 0,3446 & 0,1044 & 0,1699 & 0,1030 & 0,1282 & 0,1021 \\
\hline 1,9 & 0,2804 & 0,0738 & 0,1366 & 0,0741 & 0,1056 & 0,0741 \\
\hline
\end{tabular}

Os resultados mostrados na Tabela (9.3) apresentam boa concordância em comparação com a condição de contorno (CDC), divergindo apenas nos extremos por causa do efeito nos cantos.

O mapa de cor para deslocamentos do nó central na direção $x_{3}$ e momentos $\left(m_{x x}\right)$ 
podem ser visualizados nas Figuras 9.7 e 9.8 .

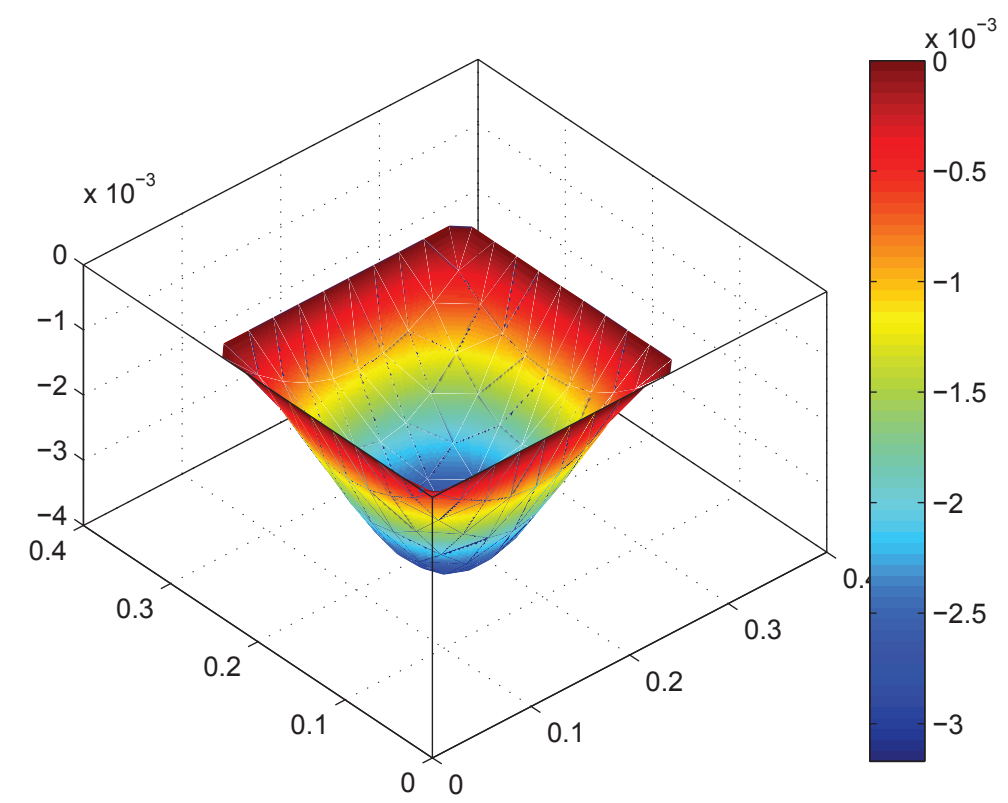

Figura 9.7: Mapa de cor do deslocamento vertical do nó central da placa de espessura $h=$ $0,0254 \mathrm{~m}$.

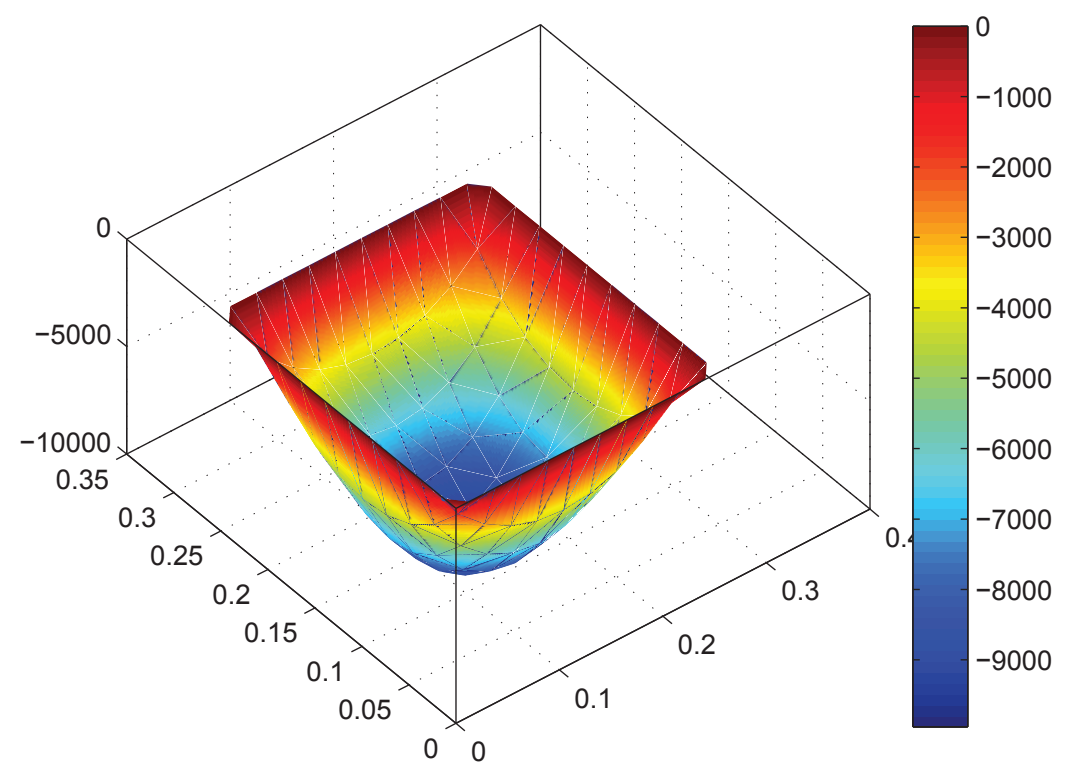

Figura 9.8: Mapa de cor do momento $\left(m_{x x}\right)$ da placa de espessura $h=0,0254 \mathrm{~m}$. 


\subsubsection{Placa quadrada engastada nos quatro lados sob carga uni- formemente distribuída}

No próximo exemplo, vamos considerar uma placa quadrada engastada com a mesma geometria e propriedades da placa analisada na seção (9.1.1). A placa é sujeita a um carregamento uniformemente distribuído (Figura 9.9).

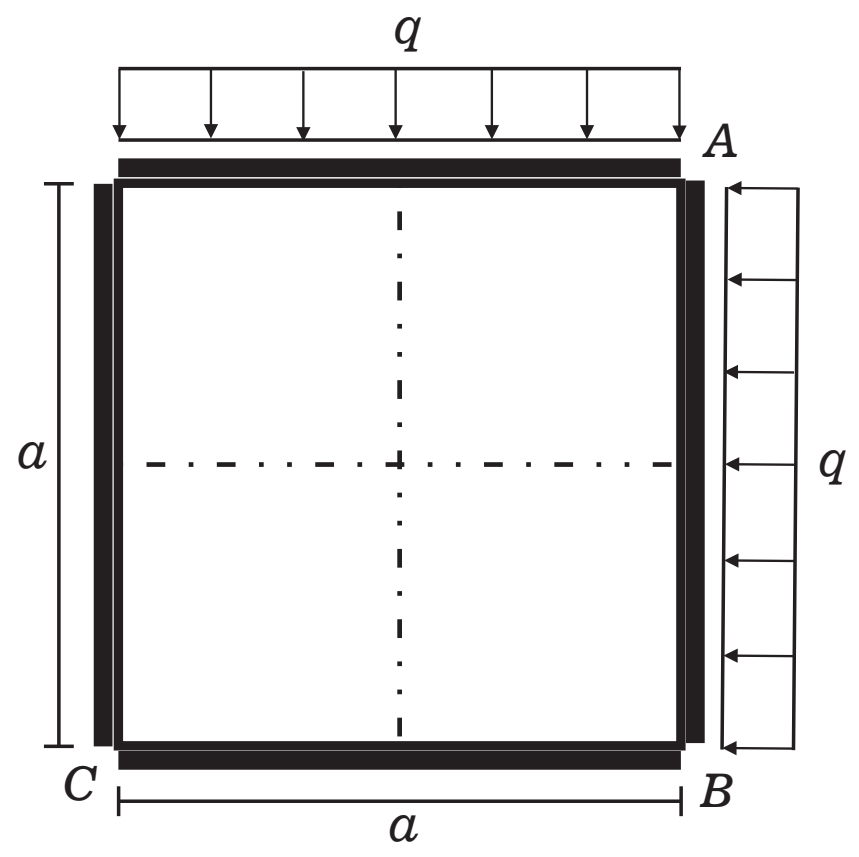

Figura 9.9: Placa quadrada engastada nos quatro lados.

A Tabela 9.4 apresenta os resultados de deslocamento vertical e momento no centro da placa engastada para as relações de espessura/lado: $h / a=0,05$ e $h / a=0,1$. Os resultados apresentam boa concordância com a literatura. Foram usados 48 elementos no contorno de mesmo comprimento (12 elementos por lado). 
Tabela 9.4: Deslocamento vertical e momentos no centro da placa engastada.

\begin{tabular}{|c|c|c|c|c|c|c|c|}
\hline \multirow{2}{*}{$h / a$} & \multicolumn{3}{|c|}{$w \times 10^{-3}(\mathrm{~m})$} & \multicolumn{3}{c|}{$m_{x x}(\mathrm{~N} . \mathrm{m})$} & $\sigma_{x x} \times 10^{8}\left(\mathrm{~N} / \mathrm{m}^{2}\right)$ \\
\cline { 2 - 8 } & MEC & Sladek (2006) & Dif. (\%) & MEC & Sladek (2006) & Dif. (\%) & MEC \\
\hline 0,05 & 6,87 & 6,74 & 0,38 & 4068,1 & 4060 & 0,19 & 1,51 \\
\hline 0,1 & 0,997 & 0,995 & 0,20 & 3983,4 & 4037,4 & 1,33 & 0,37 \\
\hline
\end{tabular}

Pode ser observado que existe uma boa concordância entre ambos os resultados. Como pode-se ver na Figura 9.10, o deslocamento vertical da placa ortotrópica é reduzido em relação a placa isotrópica. Entretanto, é observado que o momento $\left(m_{x x}\right)$ na placa ortotrópica, apresenta aumento em relação a placa isotrópica (Figura 9.11).

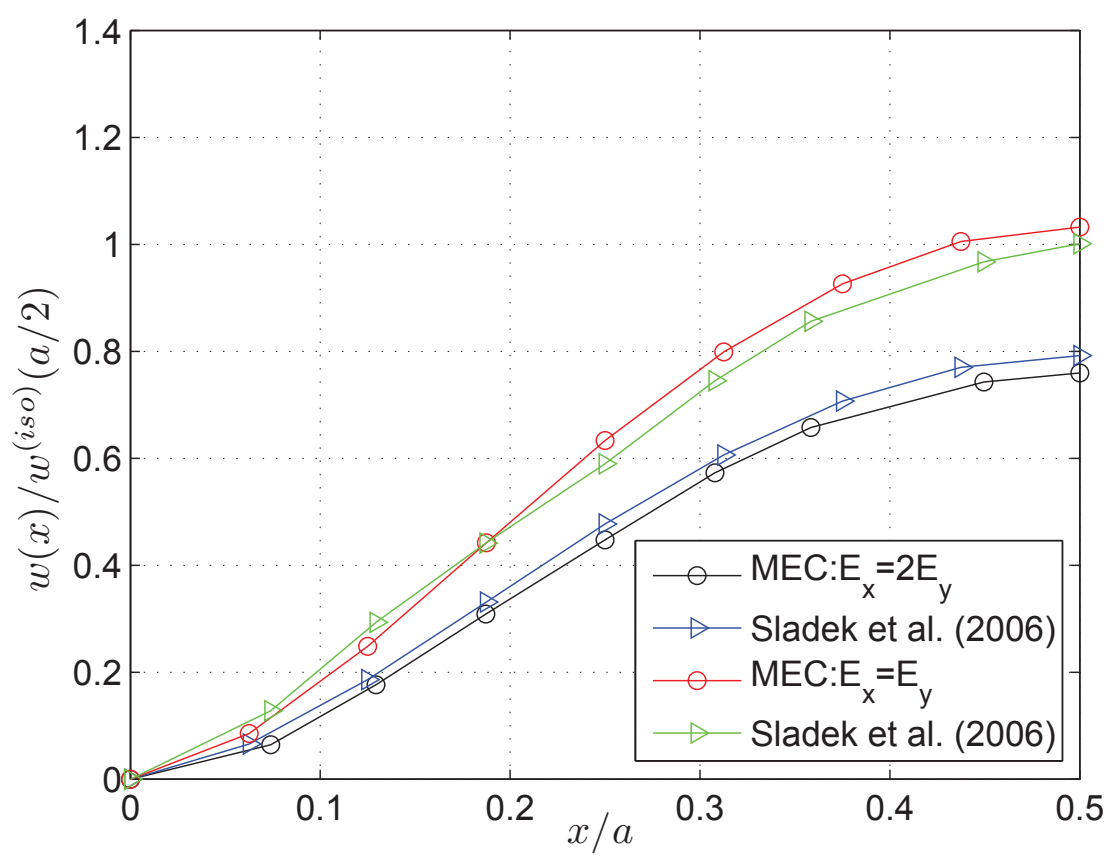

Figura 9.10: Variação do deslocamento transversal $w$ devido um carregamento estático uniformemente distribuído. 


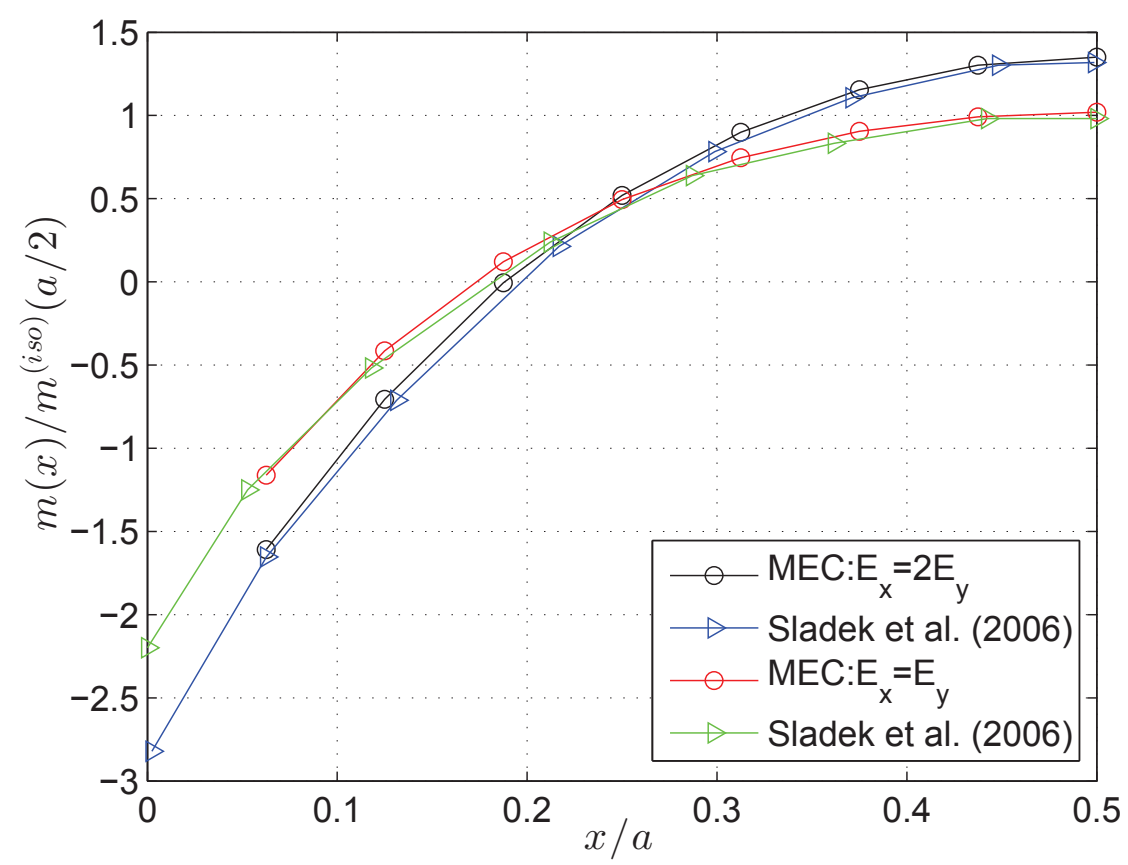

Figura 9.11: Variação do momento $m_{x x}$ devido um carregamento estático uniformemente distribuído.

Na Tabela 9.5 foram comparados os resultados para diferentes relações de espessura/lado com o método dos elementos finitos. Os resultados apresentaram boa concordância.

Tabela 9.5: Deslocamentos e esforços no centro da placa para diferentes relações $h / a$.

\begin{tabular}{|c|c|c|c|c|c|c|}
\hline \multirow{2}{*}{$h / a$} & \multicolumn{2}{|c|}{$w(\mathrm{~m})$} & \multicolumn{2}{c|}{$m_{x x} \times 10^{5}(\mathrm{~N} . \mathrm{m})$} & \multicolumn{2}{c|}{$\sigma_{x x} \times 10^{8}\left(\mathrm{~N} / \mathrm{m}^{2}\right)$} \\
\cline { 2 - 7 } & MEC & MEF & MEC & MEF & MEC & MEF \\
\hline 0,01 & 6,359 & 6,246 & 2,556 & 2,531 & 3,835 & 3,797 \\
\hline 0,02 & 0,814 & 0,787 & 2,574 & 2,574 & 9,485 & 9,654 \\
\hline 0,03 & 0,236 & 0,245 & 2,576 & 2,526 & 4,294 & 4,210 \\
\hline 0,04 & 0,105 & 0,101 & 2,572 & 2,521 & 2,411 & 2,364 \\
\hline
\end{tabular}




\subsubsection{Influência do número de elementos no contorno}

A placa foi discretizada usando-se 20, 40, 60, 80 e 100 elementos no contorno, respectivamente. Foi usado somente 1 nó interno e foram obtidos resultados para deslocamento vertical e momento do nó central.

Tabela 9.6: Valores obtidos no centro da placa engastada de espessura $h=0,0127 \mathrm{~m}$.

\begin{tabular}{|c|c|c|c|c|}
\hline \multirow{2}{*}{ MALHA } & \multicolumn{2}{|c|}{ Deslocamento } & \multicolumn{2}{c|}{ Momento } \\
\cline { 2 - 5 } & $w\left(\times 10^{-3} \mathrm{~m}\right)$ & Dif. (\%) & $m_{x x}$ (N.m) & Dif. (\%) \\
\hline 20 & 6,80 & 0,89 & 4079,90 & 2,25 \\
\hline 40 & 6,77 & 0,44 & 4069,17 & 1,98 \\
\hline 60 & 6,76 & 0,29 & 4067,15 & 1,93 \\
\hline 80 & 6,76 & 0,29 & 4066,31 & 1,91 \\
\hline 100 & 6,76 & 0,29 & 4065,87 & 1,90 \\
\hline 120 & 6,75 & 0,14 & 4065,60 & 1,89 \\
\hline Sladek $(2006)$ & 6,74 & 3990 \\
\hline
\end{tabular}

Pode-se notar na Tabela 9.6 que as diferenças nos resultados diminuem a medida que aumenta os números de nós no contorno tanto para deslocamento quanto para momento. Nota-se também que as diferenças nos resultados apresentam-se pouco significativos para deslocamentos (em torno de 0,14\%). Para momentos estabilizaram-se para a malha de 120 elementos (30 elementos por lado). 
A Figura 9.12 mostra a variação da tensão $\left(\sigma_{x x}\right)$ ao longo da linha central da placa $(y=a / 2)$, considerando uma placa isotrópica e ortotrópica. Pode-se notar que as tensões apresentam valores superiores para placas ortotrópicas.

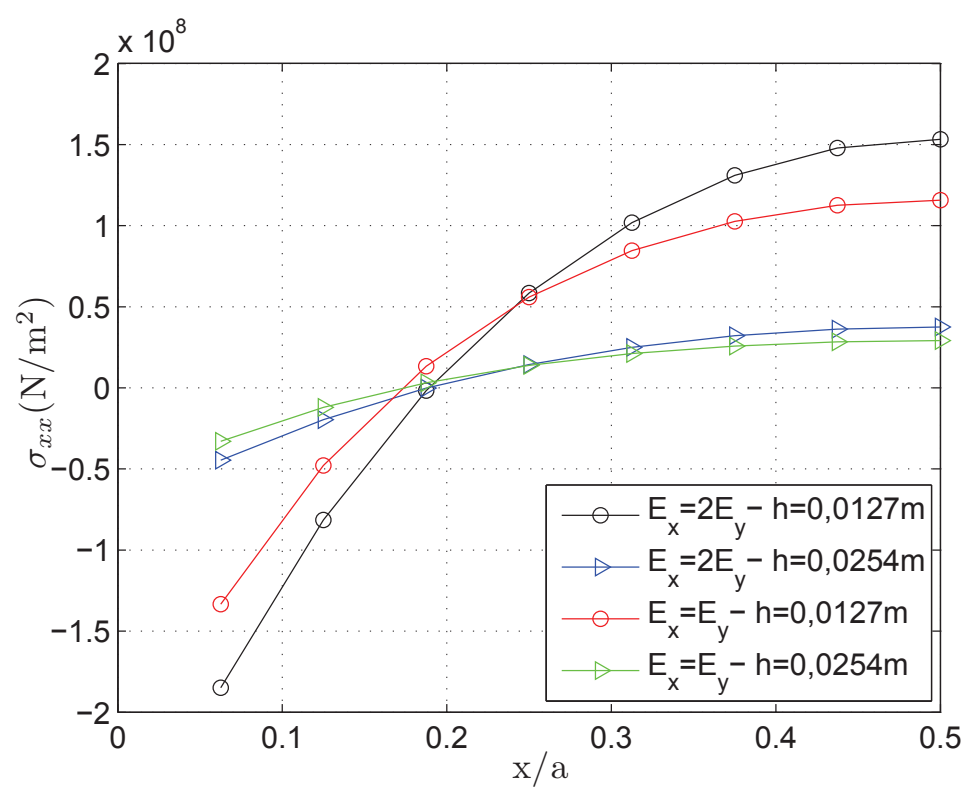

Figura 9.12: Variação da tensão $\sigma_{x x}$ devido um carregamento estático uniformemente distribuído.

Na Figuras 9.13 e 9.14 foram obtidos resultados para o calculo de momentos ao longo do contorno $A B$ da Figura 9.9. Os resultados apresentaram boa concordância com o método dos elementos finitos (MEF). 


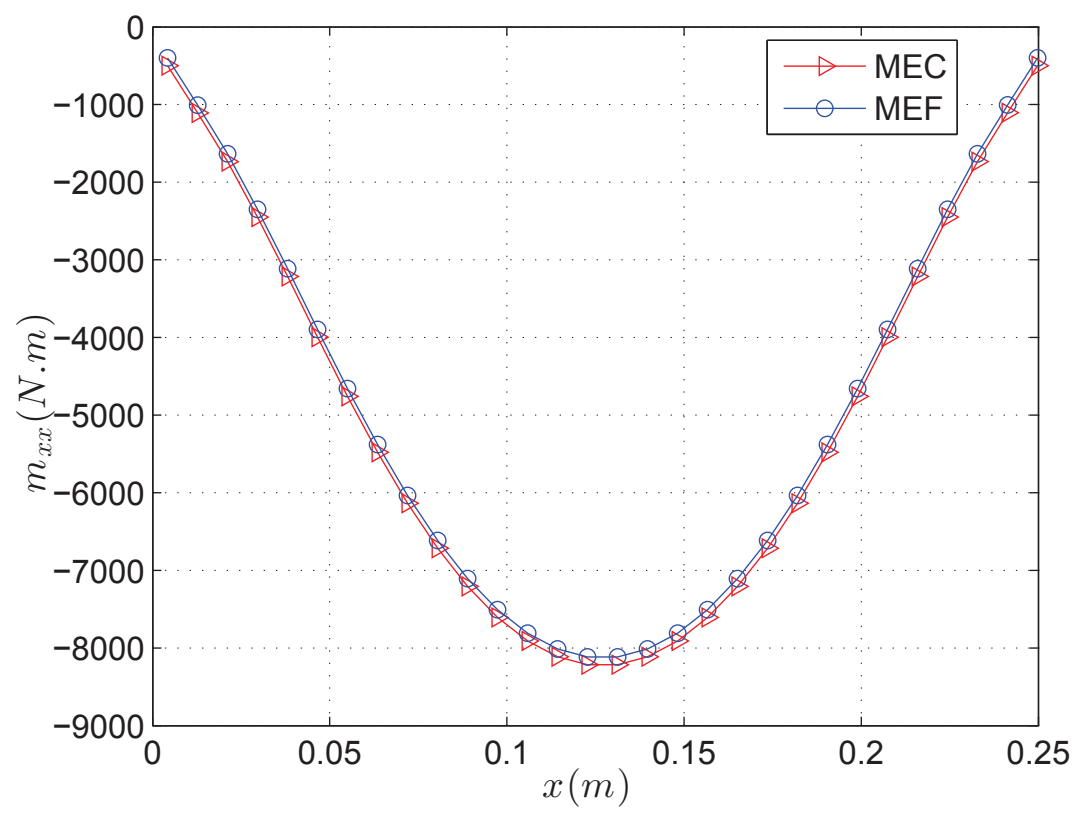

Figura 9.13: Momentos na direção $x$ ao longo do lado $A B$ da placa.

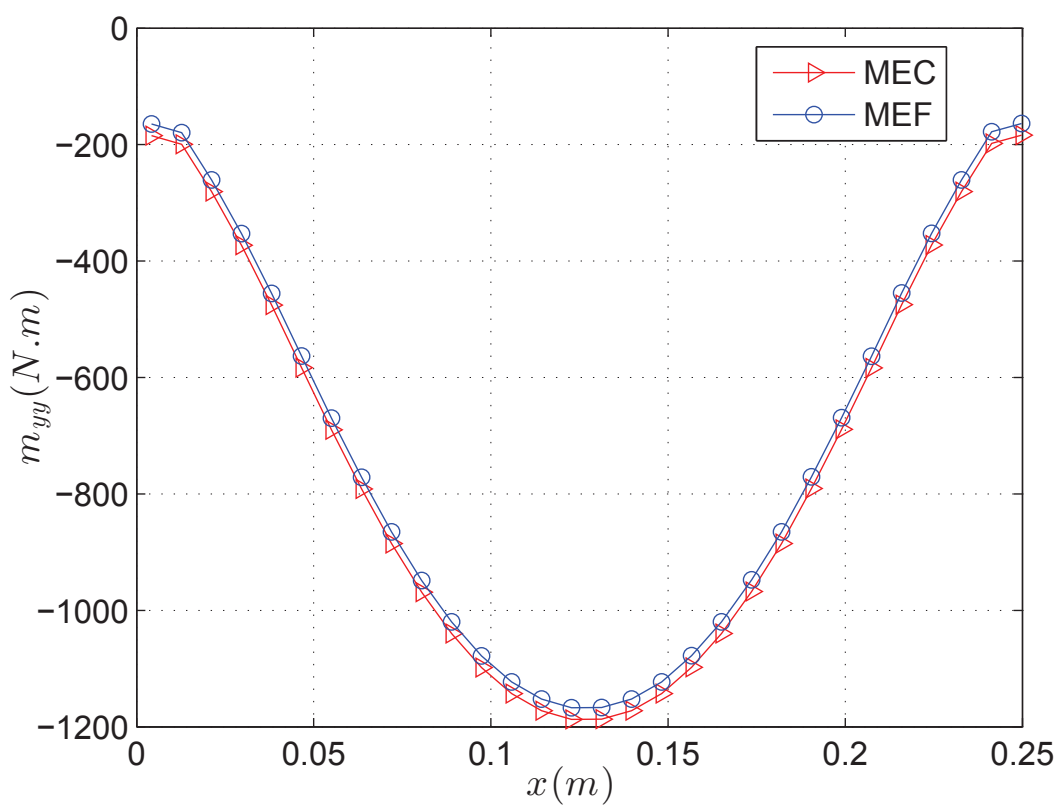

Figura 9.14: Momentos na direção y ao longo do lado $A B$ da placa.

O mapa de cor para deslocamentos do nó central na direção $x_{3}$ e momentos $\left(m_{x x}\right)$ podem ser visualizados nas Figuras 9.15 e 9.16 . 


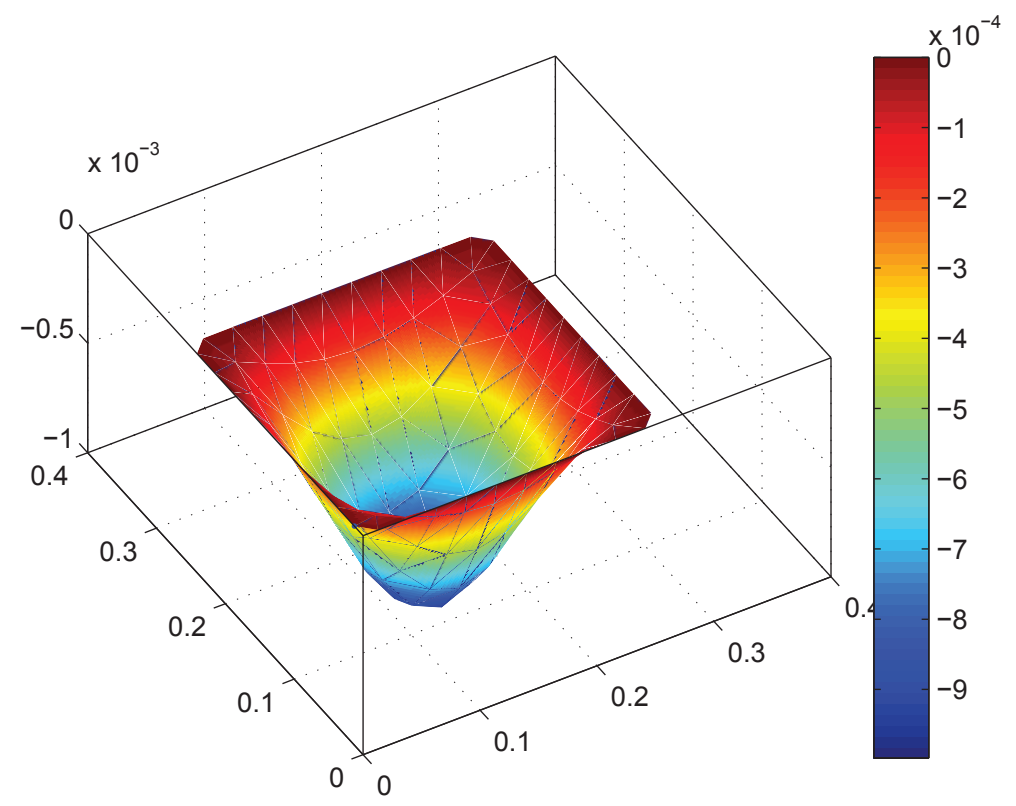

Figura 9.15: Mapa de cor do deslocamento vertical do nó central da placa de espessura $h=0,0254 \mathrm{~m}$.

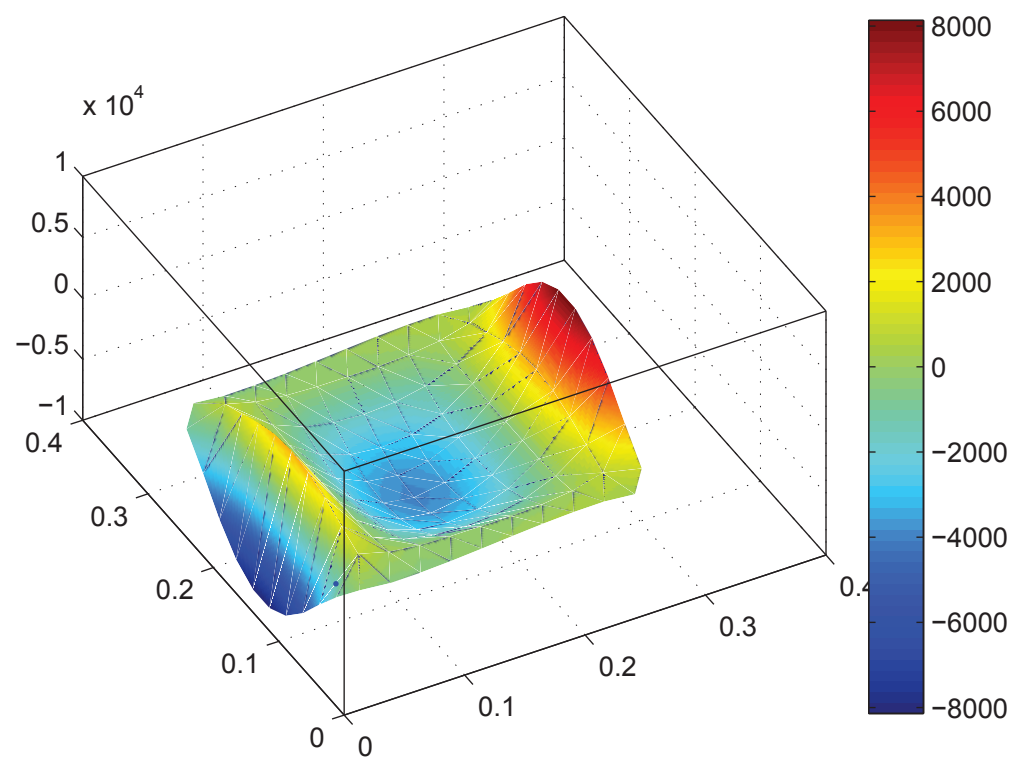

Figura 9.16: Mapa de cor do momento $\left(m_{x x}\right)$ da placa de espessura $h=0,0254 \mathrm{~m}$. 


\subsubsection{Placa quadrada com um lado engastado e os demais livres}

Considere uma placa com um lado engastados e três livres (Figura 9.17) com a mesma geometria e propriedades da placa analisada na seção (9.1.1). A placa é sujeita a um carregamento uniformemente distribuído. Foram determinados os deslocamentos, momentos e tensões ao longo da linha central $M M$ da placa.

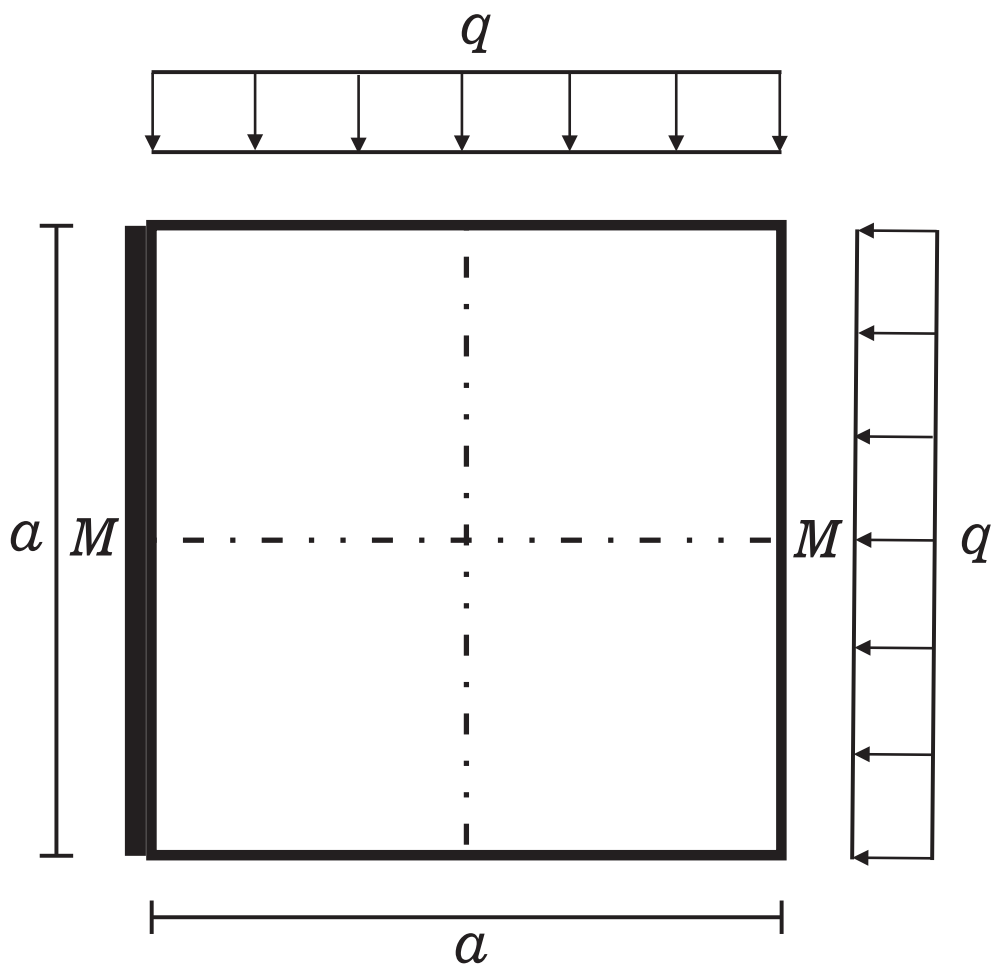

Figura 9.17: Placa quadrada com um lado engastado e três lados livres.

Os resultados foram obtidos para placa $(h=0,0127 \mathrm{~m})$ ortotrópica e isotrópica. 


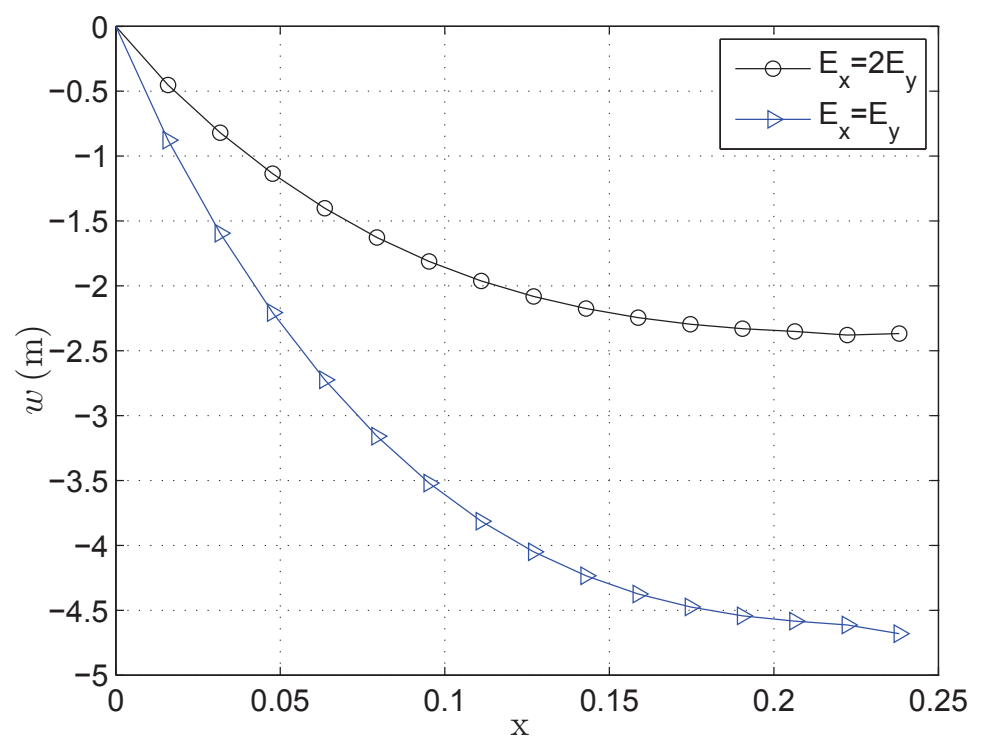

Figura 9.18: Deslocamento vertical ao longo da linha central da placa.

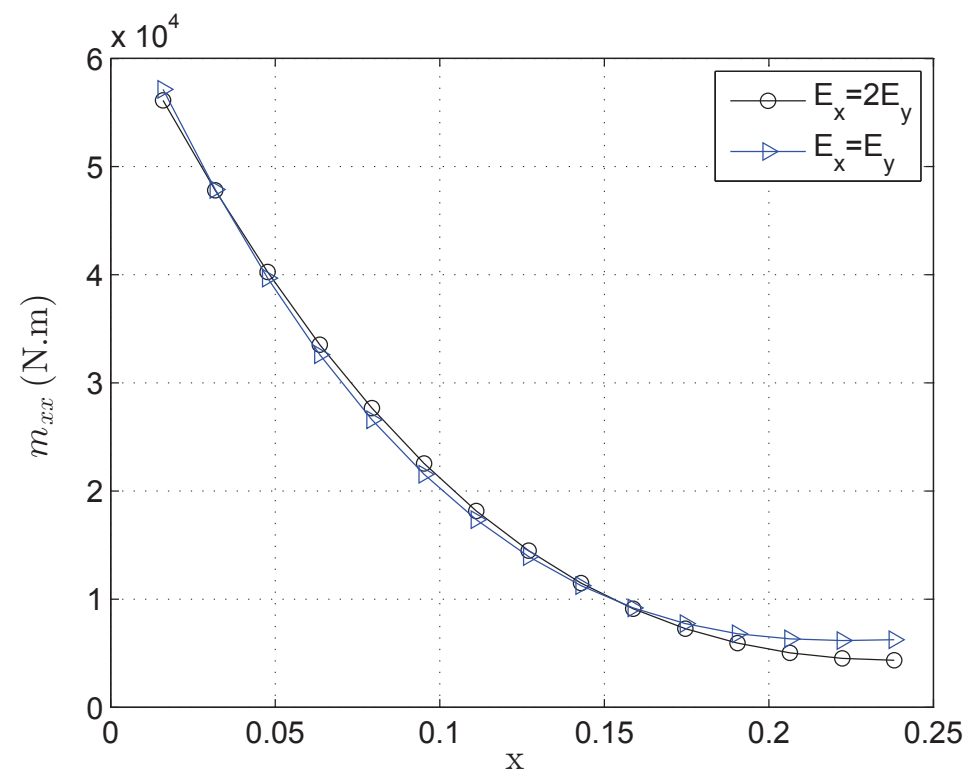

Figura 9.19: Momento $\left(m_{x x}\right)$ ao longo da linha central da placa. 


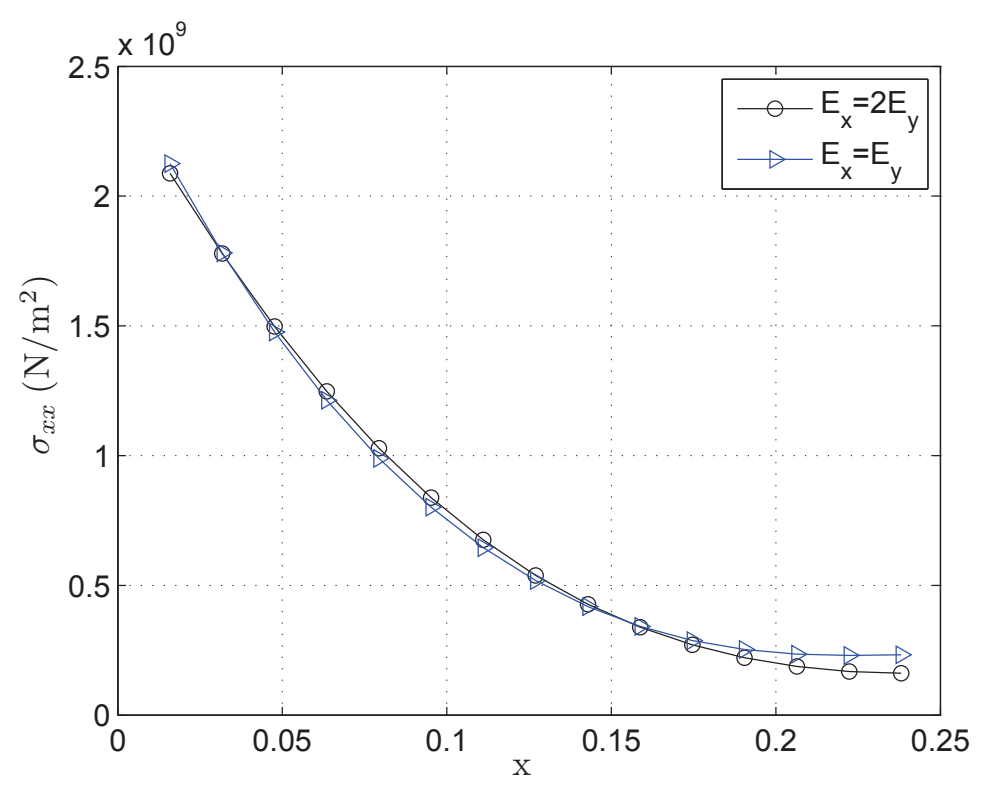

Figura 9.20: Tensões $\left(\sigma_{x x}\right)$ ao longo da linha central da placa.

Pode-se notar na Figura 9.18 que os deslocamentos são menores na placa ortotrópica, ou seja, $E_{x}=2 E_{y}$. Os momentos e tensões mostrados nas Figuras 9.19 e 9.20, respectivamente, apresentam poucas variações nos resultados ao longo da linha $M M$, pois não dependem das propriedades do material. 


\section{Capítulo 10}

\section{Conclusões}

\subsection{Considerações finais}

Neste trabalho foi desenvolvida uma formulação do método dos elementos de contorno para análise de flexão em problemas estáticos para placas isotrópicas e ortotrópicas.

Foi apresentada uma revisão sobre materiais compósitos e suas aplicações. A teoria de laminados foi apresentada desconsiderando o efeito do tempo de ensaio e temperatura. A teoria de elasticidade linear para materiais isotrópicos foi apresentada. Foram apresentadas as equações de equilíbrio para placas em coordenadas cartesianas e cilíndricas.

A formulação do método dos elementos de contorno para análise de placas espessas isotrópicas e ortotrópicas de materiais compósitos considerando o efeito da deformação por cisalhamento na direção transversal. A solução fundamental para placas isotrópicas e ortotrópicas foram obtidas através do operador de Hörmander e a transformada de Radon. A transformada de Hörmander foi utilizada para transformar o conjunto formado pelas equações diferenciais parciais de equilíbrio em uma única equação diferencial parcial e em seguida foi usado a transformada de Radon para transformar a equação diferencial parcial em uma equação diferencial ordinária. A equação diferencial ordinária foi resolvida utilizando o método da variação dos parâmetros. 
Para o cálculo de momentos e esforços cisalhantes em pontos internos e contorno de placas isotrópicas e ortotrópicas, os momentos foram calculados derivando-se a equação integral de deslocamentos em relação ao ponto fonte e substituindo os esforços generalizados de momentos e esforços cisalhantes, respectivamente. A equação integral de momentos e esforços cisalhantes para pontos no contorno foram obtidas de forma similar para um ponto localizado no contorno suave.

Para o cálculo das tensões faz-se o uso das relações propostas por Reissner onde as tensões ao longo da espessura variam de forma linear e as tensões devido as esforços cortantes variam de forma parabólica.

Os kernels presentes na equação integral de momentos e esforços cisalhantes são obtidos usando a derivada da solução fundamental. O tratamento dos kernels fortemente singulares e hipersingulares foram realizados utilizando a quadratura de Gauss e Telles para os kernels de deslocamentos e a quadratura de Campos e Albuquerque (2013) para os kernels de forças. Para quadratura de Gauss e Telles foram utilizandos quatro pontos de integração e a quadratura de Campos e Albuquerque (2013) foram utilizados seis pontos de integração.

As integrais de domínio foram transformadas em integrais de contorno usando o método de integração radial (MIR). Com isso, em nenhum momento houve a necessidade de discretização do domínio, resguardando a principal característica do MEC que é a discretização apenas do contorno. Foram usados elementos constantes na discretização de todas as formulações apresentadas afim de simplificar a modelagem matemática.

Notou-se em todos os resultados que os nós internos não apresentam influência nos resultados. Em geral, os resultados mostraram boa concordância com os resultados disponíveis na literatura e softwares comerciais.

\subsection{Trabalhos Futuros}

Ficam como propostas para trabalhos futuros: 
- Estender a formulação para análise dinâmica;

- Estender a formulação para grandes deformações;

- Estender a formulação para danos;

- Desenvolver procedimentos para tornar menor o custo computacional do MIR. 


\section{Referências}

Agarwal, B. D. and Broutman, L. J. (1990). Analysis and performance of fiber composites. 2nd Edition, John Wiley and Sons, Inc, New York.

Albuquerque, E. L., Sollero, P., and Aliabadi, M. H. (1999). The dual boundary element formulation applied to dynamic fracture mechanics in anisotropic materiails. International Conference on Boundary Element Techniques. Londres: EC LTD, p. 23-39.

Albuquerque, E. L., Sollero, P. Venturini, W. and Aliabadi, M. H. (2006). Boundary element analysis of anisotropic kirchhoff plates. International Journal of Solids and Structures, v. 43 , p. $4029-4046$.

Albuquerque, E. L. (2001). Análise de problemas dinâmicos em materiais anisotrópicos usando o método dos elementos de contorno. Tese de Doutorado, Faculdade de Engenharia Mecânica, Universidade Estadual de Campinas.

Albuquerque, E. L., Santana, A. P. and Sollero, P. (2007). On the accuracy of the radial integration method applied to the modal analysis of thin anisotropic plates. International Conference on Boundary Element Techniques. Londres: EC LTD, p. 13-18.

Albuquerque, E. L., Sollero, P. and Aliabadi, M. H (2002). The boundary element method applied to time dependent problems in anisotropic materials. International Journal of Solids and Structurres, v. 39, p. 1405-1422.

Albuquerque, E. L., Sollero, P., and Fedelinski, P. (2002). Free vibration analysis of anisotropic material structurres using the boundary element method. Engineering Analysis with Booundary Elements, v. 27, p. 977-985. 
Albuquerque, E. L., Sollero, P., and Fedelinski, P. (2004). Dual booundary element method for anisotroppic dynamic fracture mechanics. International Journal for Numerical Method in Engineering, v. 59, p. 1187-1205.

Albuquerque, E. L., Sollero, P., Venturini W. S. and Aliabadi, M. H. (2006). Boundary element analysis of anisotropic Kirchhoff plates. International Journal of Solids and Structures, v. 43, p. 4029-4046.

Albuquerque, E. L., Sollero, P., and Paiva, W. P. (2006). The radial integration method applied to dynamic problems of anisotropic plates. Communications in Numerical Methods in Engineering, v. 23, p. 805-818.

Aliabadi, M. H. (2002). The Boundary Element Method: Applications in Solids and Structures. John Wiley e Sons, New York, 1st edition.

Aliabadi, M. H. and Sollero, P. (2002). Crack growth analysis in homogeneous orthotropic laminates. Composite Science and Technology, v. 58, p. 1697-1703.

Aliabadi, M. H. and Sollero, P. (1998). Crack growth analysis in homogeneous orthotropic laminates. Composites Science and Technology, v. 58, p. 1697-1703.

Atluri, S.N. and Shen, S. (2002). The meshless local Petrov-Galerkin (MPLG) method.Tech Science Press, Los Angels, CA.

Abramowitz, M. and Stegun, I.A. (1965). Handbook of Mathematical Functions with Formulas, Graphs, and Mathematical Tables. New York: Dover Publications.

Barcellos, C.A. and Westphalm T. (1992). Reissner/Mindlin's plate models and the boundary element method. Boundary Element Technology VII, p. 589-604.

Bares, R. (1969). Tables pour le Calcul des Dalles et des Parois. Paris, Dunod.

Brebbia, C. A. and Nardini, D. (1982). A new approach to free vibration analysis using boundary elements. Applied Mathematical Modelling, v.7, p. 312-326.

Brebbia, C. and Dominguez, J. (1989). Boundary Element: An Introdutory Course. Computational Mechanics Publications, Southampton, 2nd edition. 
Bezine, G. (1978). Boundary integral formulation for plate flexure with arbitrary boundary conditions. Mechanics Research Communications, v. 5, p. 197-206.

Betti, E. (1872). Teoria dell elasticita. II Nuovo Ciemento, p. 7-10.

Somigliana, C. (1885). Sopra l'equilíbrio di un corpo elastico isotropo. II Nuovo Ciemento, p. $17-20$.

Cody, W.J., Henry, C. and Thacher, Jr. (1969). Chebyshev Aprroximations for the Exponential Integral Ei(x). Mathematics of Computation, v. 23, p. 289-303.

Courbon, J. (1979). Stress in Plates and Shells. Eyrolles, Paris.

Cruze, T. A. (1969). Numerical solutions in three-dimensional elastostatics. International Journal of Solids and Structures, v. 5, p. 1259-1274.

Campos, L. S. (2012) Análise de Tensões em Placas Finas Laminadas sob Carregamento Dinâmico usando o Método dos Elementos de Contorno. Dissertação de Mestrado, Faculdade de Tecnológia, Universidade de Brasília, 2012.

Campos, L. S. and Albuquerque, E. L. (2013). Quadrature Gauss to treated singularities of type $1 / r$ and $1 / r^{2}$. College of Techonology, University of Brazilia.

Campos, L. S., Sousa, K. R. P. ; Santana, A. P., Reis, A. ; Albuquerque, E. L. and Sollero, P. (2011). Stress analysis of thin plate composite materials under dynamic loads using the boundary element. International Conference on Boundary Element and Meshless Techniques XII, p. 190-195.

Deans, S. (1993). The Radon transform and some of its applications. Krieger Publishing Company, Malabar, Flórida.

Dias Júnior, A. B., Albuquerque, E. L. and Fortaleza, E. (2013). The boundary element method applied to the analysis of fluid extraction from from a reservoir. International Conference on Boundary Element and Meshless Techniques XIV, p. 190-194.

Dinis, L. M. J. S. (2004). Placas e Cascas. Apostila da Seção Mecânica Aplicada, Faculdade de Engenharia da Universidade do Porto. 
Dym, C.L. and Shames, I.H. (1973). Solid Mechanics: A Variational Approach. McGraw-Hill.

Deb, A. (1996). Boundary elements analysis of anisotropic bodies under thermo mechanical body force loadings. Computers and Structures, v. 58, p. 715-726.

Dominguez, J. (1993). Boundary elements in dynamics. Computational Mechanics Publication, Southampton, Boston.

El-Zafrany, A., Fadhil, S., and Debbih, M. An efficient approach for boundary element bending analysis of thin and thick plates. Computers and Structures, v. 56, p. 565-576.

Flamínio, L. N. e Pardini, L. C. (2006). Compósitos Estruturais: ciência e tecnológia. Edgard Blücher, São Paulo.

Gaul, L., Kögl, M. and Wagner, M. (2003). Boundary Element Methods for Engineers and Scientits. Berlin: Springer-Verlag.

Gao, X. (2002). The radial integration method for evaluation of domain integrals with boundary only discretization. Engineering Analysis with Boundary Elements, v. 26, p. 905916.

Gel'fand, I. M., Graev, M. I. and Vilenkin, N. Y. (1966). Generalized Functions. New York: Academic.

Gibson, R. F. (1994). Principles of composite material mechanics. McGraw-Hill, New York.

Golberg M.A., Chen C.S. and Bowman H. (1999). Some recent results and proposals for the use of radial basis functions in the BEM. Engineering Analysis with Boundary Element, v. 23, p. 285-296.

Greenberg, M.D. (1971). Application of Green's Functions in Science and Engineering. Prentice-Hall Inc.

Gouvêa, A. R. (2006). Critérios de falha e otimização de estruturas de materiais compósitos usando o método dos elementos de contorno. Dissertação de Mestrado, Faculdade de Engenharia Mecânica, Universidade Estadual de Campinas. 
Gel'fand, I. M. and Shilov, M. A. (1967). Generalised function. Vol. 1, Academic Press, Inc. New York.

Holmes, M. and Just, D. J. (1983). GRP in structural engineering. London: Applied Science Publishers.

Hull, D. C. and Clyne, T. W. (1996). An introduction to composite materials. Cambridge, UK: Cambridge University Press.

Kane, J. A. (1994). Boundary Element Analisys in Engineering Continuum Mechanics. Prentice Hall, New Jersey.

Kirchhoff, G. R. (1950). On the equilibrium and motion of an elastic plate. Journal of Mathematical Sciences, v. 40, p. 51-58.

Kolm, P. and Rokhlin, V. (2001). Numerical Quadratures for Singular and Hypersingular Integrals. Computers and Mathematics with Applications, v. 41, p. 327-352.

Kogl, M. and Gaul, L. (2000a). A boundary element method for transient piezoeletric analysis. Engineering Analysis with Boundary Element, v. 24, p. 591-598.

Kogl, M. and Gaul, L. (2000b). A 3-d doundary element method for dynamic analysis of anisotropic elastic solids. CMES: Computer Modeling in Engineering and Sciences, v. 1, p. 27-43.

Kogl, M. and Gaul, L. (2003). Free vibration analysis of anisotropic solids with the boundary element method. Engineering Analysis with Boundary Element, v. 27, p. 107-114.

Kupradze, V. D. (1965). Potential methods in the theory of elasticity. Jerusalém: Israel Program for Scientific Translation, 339p.

Lachat, J. A. (1975). A further development of the boundary integral technique for elastostatic. University of Southampton.

Lekhnitskii, S. G. (1963). Anisotropic plates. Gordon and Breach, New York. 
Loeffler, C. F. e Mansur, W. J. (1986). Vibrações Livres de Barras e Membranas Através do Método dos Elementos de Contorno. Revista Brasileira de Engenharia (RBE), Caderno de Engenharia Civil, v. 4, p. 5-23.

Marczak, R. J. and Creus, G. J. (2002). Direct evoluation of singular integral in boundary element analysis of thick plates. Engineering Analysis with Boundary Elements, v. 26, p. 653-665.

Mansfield, E.H. (1989). The Bending and Stretching of Plates. Cambridge University Press, $228 \mathrm{p}$.

Mindlin, R. D. (1951). Influence of rotatory inertia and shear on flexural motions of isotropic elastic plates. Journal of Applied Mechanics, v. 18, p. 31-38.

Pilkey, W and Wunderlich, W. (1994). Mechanics of structures: variational and computational methods. Inc Boca Raton, Florida: CRC Press.

Paiva, J. B. (1987). Boundary element formulation for plate bending and its aplication in engineering. Tese de Doutorado, Escola de Engenharia de São Carlos, Universidade de São Paulo.

Paiva, W. P. (2005). Análise de problemas estáticos e dinâmicos em placas anisotrópicas usando o método dos elementos de contorno. Tese de Doutorado, Faculdade de Engenharia Mecânica, Universidade Estadual de Campinas.

Paiva, W. P., Sollero, P. and Albuquerque, E. L. (2003). Treatment of hypersingularities in boundary element anisotropic plate bending problems. Latin American Journal of Solids and Structures, v. 1, p. 49-73.

Palermo Junior, L. (2000). A análise elástica de placas e o método dos elementos de contorno. Tese de Livre Docência, Faculdade de Engenharia Civil, Universidade Estadual de Campinas, 2000.

Poisson, S. D. (1829). Memoire sur l'equilibre et le mouvement des corps solides. Journal of Mathematics Physics, v. 8, p. 357?570. 
Portela, A., Aliabadi, M. H., and Rooke, D. P. (1992a). Dual boundary element method: Effcient implementation for cracked problems. International Journal for Numerical Methods in Engineering, v. 33, p. 1269-1287.

Portela, A., Aliabadi, M. H., and Rooke, D. P. (1992b). Dual boundary element analysis of cracked plates: singularity subtraction technique. International Journal of Fracture, v. 55 , p. $17-28$.

Pouzada, E. V. S. (1999). Estudo da aplicação do métodos dos elementos de contorno à análise de propagação de estruturas guiantes. Tese de Doutorado. Escola Politécnica, Universidade de São Paulo.

Paiva, W. P., Sollero, P., e Albuquerque, E. L. (2003). Treatment of hypersingularities in boundary element anisotropic plate bending problems. Latin American Journal of Solids and Structures, v. 1, p. 49-73.

Rajamohan, C. and Raamachandran, J. (1999). Bending of anisotropic plates by charge simulation method. Advances in Engineering Software. v. 30, p. 369-373.

Rizzo, F. J. (1967). An integral equation approach to boundary value problems of classical elastostatics. Quaterly of Applied Mathematics, v. 25, p. 83-95.

Ricardella, P. (1973). An implementation of the boundary integral technique for planar problems in elasticity and elasto-plasticity. Carnegie-Mellon University.

Rezende, M. R. e Botelho, E. C. (2008). O uso de compósitos estruturais na insdústria aeroespacial. Centro Técnico Aeroespacial, Instituto de Aeronáutica e Espaço.

Rashed, Y. F. (1999). Boundary method formulation for thick plates. Topics in Engineering, WITpress Southampton, Boston.

Reis, A. (2010) Formulações do Método dos Elementos de Contorno para Análise de Placas de Materiais Compósitos Laminados. Tese de Doutorado, Falcudade de Engenharia Mecânica, Universidade Estadual de Campinas. 
Reis, A., Albuquerque, E. L., Torsani, L. F., Palermo, L. J. and Sollero, P. (2011). Computation of moments and stresses in laminated composite plates by the boundary elements method. Engineering Analysis with Boundary Elements, v. 35, p. 105-113.

Reis, A., Albuquerque, E.L. and Palermo, J.L. (2013) The boundary element method applied to orthotropic shear deformable plates. Engineering Analysis with Boundary Elements, v. 37, p. $738-746$.

Reissner, E. (1947). On bending of elastic plates. Quarterly of Applied mathematics, v. 5, p. $55-68$.

Schclar, N. A. and Partridge, P. W. (1993). 3D anisotropic elasticity with bem using the isotropic fundamental solution. Engineering Analysis with Boundary Elements, v. 11, p. $137-144$.

Sanches, L. C. F. (1998). Uma resolução de placas com a teoria de Mindlin através do método dos elementos de contorno. Dissertação de Mestrado, Faculdade de Engenharia Civil, Universidade Estudual de Campinas.

Shi, G. and Bezine, G. (1988). A general boundary integral formulation for the anisotropic plate bending problems. Journal Composite Materials, v. 22, p. 694-716.

Sladek, J., Sladek, V., Zhang, Ch., Krivacek, J. and Wen, P. H. (2006). Analysis of ortotropic thick plates by meshless local Petrov-Galerkin (MLPG) method. International Journal for Methods in Engineering, v. 67, p. 1830-1850.

Somigliana, C. (1885). Sopra l'equilibrio di corpo elastico isotropo. Nuovo Cimento, p. 17-20.

Sollero, P. and Aliabadi, M. H. (1995). Anisotropic analysis of composite laminates using the dual boundary elements methods. Composite Structures, v. 31, p. 229-234.

Stern, M. (1979). A general boundary integral formulation for the numerical solution of plate bending problems. International Journal of Solids and Structures, v. 15, p. 769-782.

Schclar, N. A. (1994). Anisotropic analysis using boundary elements. Computational Mechanics Publications, Southampton, Boston. 
Telles, J. C. F. (1987). A self-adaptive co-ordinate transformation for eficient numerical evaluation of general boundary element integrals. International Journal for Numerical Methods in Engineering, v. 24, p. 959-973.

Timoshenko, S. and Woinowsky-Krieger, S. (1959). Theory of plates and shells. McGraw-Hill, New York.

Timoshenko, S. and Goodier, J.N. (1934). Theory of Elasticity. McGraw-Hill, New York.

Torsani, F. L. (2007). Implementação do Cálculo das Tensões em Placas Compósitas Laminadas usando o Método dos Elementos de Contorno. Dissertação de Mestrado, Universidade Estadual de Campinas.

Telles, J.C.F. (1987). A self-adaptive coordinate transformation for efficient numerical evaluation of general boundary element integrals. Int. J. Num. Methods Engineering, v. 24, p. 959-973.

Ugural, A. C. (1981). Stress in Plates and Shells. McGraw-Hill Book Comp.

Venturini, W. S. (1988). A study of boundary element method and it application on engineering problems. Tese de Doutorado, Universidade de São Paulo, Faculdade de Engenharia de São Carlos.

Vander Weeën, F. (1982). Application of the boundary integral equation method to Reissner's plate model. International Journal for Numerical Methods in Engineering, v. 18, p. 1-10.

Wang, J. and Huang, M. (1991). Boundary element method for orthotropic thick plates. Acta Mechanica Sinica, v. 7, p. 258-266.

Wang, J. and Schweizerhof, K. (1995). The fundamental solution of moderately thick laminated anisotropic shallow shells. International Journal of Engineering Science, v. 33, p. 995-1004.

Wang, J. and Schweizerhof, K. (1996). Study on free vibration of moderately thick orthotropic laminated shallow shells by boundary-domain elements. Applied Mathmatical Modelling, v. 20 , p. $579-584$. 
Wrobel, L. C. (2002). The Boundary Method: Applicattions in Thermo-Fluids and Acoustics. John Wiley and Sons, New York, 1st edition.

Wu, B. C. (1980). A new method for solution of anisotropic thin-plate bending porblems. PhD thesis, Michigan State University.

Wu, B. C. and Altiero, N. J. (1981). A new numerical method for the analysis of anisotropic thin-plate bending problems. Computer Methods in Applied Mechanics and Engineering, v. 25 , p. $343-353$.

Zhao, Z. (1995). On the calculation of boundary stress in boundary elements.Engineering Anlysis with Boundary Elements, v. 16, p. 317-22.

Zhao, Z. and Lan, S. (1999). Boundary stress calculation-comparasion study. Computers and Structures, v. 71, p. 77-85.

Young, W. C. and Budynas, R. G. (2001). Roark's formulas for stress and strain. 7th Edition, McGraw-Hill, New York. 


\section{Apêndice A}

\section{Método de Hörmander}

O método de Hörmander, desenvolvido por Hömander (1963), é um método geralmente usado para decompor a matriz de operadores diferenciais em um simples operador diferencial escalar a partir do qual a solução fundamental poderá ser facilmente obtida. Em outras palavras, ele transforma um sistema de equações diferenciais em apenas uma equação diferencial parcial. Para demonstrar o procedimento geral do método de Hörmander, considera-se a seguinte equação diferencial:

$$
L U^{*}=\delta
$$

onde $\delta$ é um operador diferencial.

O método de Hörmander consiste das seguintes etapas:

- Calcula-se o operador adjunto: $L^{a d j} U^{*}=-\delta$;

- Calcula-se a matriz de cofatores do operador adjunto: ${ }^{c o} L^{a d j}$;

- Calcula-se a matriz dos cofatores transposta: $\left({ }^{c o} L^{a d j}\right)^{T}$;

- Calcula-se o determinante da matriz de cofatores transposta: $\operatorname{det}\left[\left({ }^{c o} L^{a d j}\right)^{T}\right]$; 
- Calcula-se o escalar potencial $\phi$ o qual é a solução da seguinte equação:

$$
\operatorname{det}\left[\left({ }^{c o} L^{a d j}\right)^{T}\right] \phi=-\delta ;
$$

- Calcula-se a solução fundamental usando a seguinte equação: $U^{*}={ }^{c o} L^{a d j} \phi$.

O operador de Hörmander representa a solução fundamental $U^{*}$ como um vetor de derivadas de um escalar potencial $\phi$.

\section{A.1 Exemplo do uso do operador de Hörmander}

Considere a equação diferencial de Navier:

$$
L_{i j} U_{k j}^{*}=-\delta(\xi, x) \delta_{k i}
$$

onde

$$
L_{i j}=G \nabla^{2} \delta_{i j}+\frac{G}{1-2 \nu} \partial_{i} \partial_{j}
$$

$\mathrm{Ou}$

$$
L=\left[\begin{array}{cc}
G \nabla^{2}+\frac{G}{(1-2 \nu)} \partial_{1} \partial_{1} & \frac{G}{(1-2 \nu)} \partial_{1} \partial_{2} \\
\frac{G}{(1-2 \nu)} \partial_{2} \partial_{1} & G \nabla^{2}+\frac{G}{(1-2 \nu)} \partial_{2} \partial_{2}
\end{array}\right]
$$

o qual representa o operador diferencial original do problema. G é o módulo de cisalhamento e $\nu$ é o coeficiente de Poisson. Este operador é seu próprio operador adjunto, assim:

$$
L_{i j}^{a d j}=L_{i j}
$$


Segundo Hörmander, a matriz de cofator do operador adjunto pode ser obtido a partir de:

$$
{ }^{c o} L^{a d j}=\left[\begin{array}{cc}
G \nabla^{2}+\frac{G}{(1-2 \nu)} \partial_{2} \partial_{2} & -\frac{G}{(1-2 \nu)} \partial_{2} \partial_{1} \\
-\frac{G}{1-2 \nu} \partial_{1} \partial_{2} & G \nabla^{2}+\frac{G}{1-2 \nu} \partial_{1} \partial_{1}
\end{array}\right]
$$

e em notação indicial:

$$
{ }^{c o} L_{i j}^{a d j}=\frac{G}{(1-2 \nu)}\left[2(1-\nu) \delta_{i j} \nabla^{2}-\partial_{i} \partial_{j}\right]
$$

A transposta da matriz de cofator pode ser escrita como:

$$
\left({ }^{c o} L_{i j}^{a d j}\right)^{T}={ }^{c o} L_{i j}^{a d j}
$$

e o determinante poderá ser calculado a partir de:

$$
\begin{aligned}
\operatorname{det}\left|\left({ }^{c o} L_{i j}^{a d j}\right)^{T}\right|=\left(G \nabla^{2}+\right. & \left.\frac{G}{(1-2 \nu)} \partial_{2} \partial_{2}\right)\left(G \nabla^{2}+\frac{G}{(1-2 \nu)} \partial_{1} \partial_{1}\right) \\
& -\left(-\frac{G}{(1-2 \nu)} \partial_{2} \partial_{1}\right)\left(-\frac{G}{(1-2 \nu)} \partial_{1} \partial_{2}\right)
\end{aligned}
$$

sendo que:

$$
\partial_{i} \partial_{j}=\partial_{j} \partial_{i}
$$

e

$$
\nabla^{2}=\partial_{1} \partial_{1}+\partial_{2} \partial_{2}
$$

Da equação (A.9), tem-se:

$$
\operatorname{det}\left|\left({ }^{c o} L_{i j}^{a d j}\right)^{T}\right|=\frac{2 G^{2}(1-\nu)}{(1-2 \nu)} \nabla^{4}
$$


De acordo com Hörmander, precisa-se obter um escalar $\Phi$ que satisfaça a seguinte equação:

$$
\frac{2 G^{2}(1-\nu)}{(1-2 \nu)} \nabla^{4} \Phi(\xi, x)=-\delta(\xi, x)
$$

A solução particular de $\Phi$ pode ser obtida a partir de:

$$
\Phi=\frac{-(1-2 \nu)}{2 G^{2}(1-\nu)} \frac{1}{8 \pi} r^{2} \log (r)+f
$$

onde

$$
f=a r^{2}+b(\log (r))+c
$$

onde, $a, b, c$ são constantes arbitrárias.

A expressão conhecida como solução fundamental de Kelvin, obtida fazendo $U_{i j}^{*}={ }^{c o}$ $L_{i j}^{a d j} \Phi$, pode ser escrita como:

$$
U_{i j}^{*}=\frac{1}{8 \pi G(1-\nu)}\left\{-\delta_{i j}(3-4 \nu) \log (r)+r_{, i} r_{, j}\right\}
$$




\section{Apêndice B}

\section{Transformada de Radon}

A mais apropriada unificação entre a matemática e a grande classe de problemas de reconstrução (problemas nos quais se quer determinar algumas propriedades da estrutura interna de um objeto sem ter que cortar ou quebrar) é a transformada de Radon num espaço Euclidiano. Há diversos campos onde se é aplicada a teoria da transformada de Radon: ótica, astronomia, geofísica, biologia molecular e principalmente na medicina, com a tomografia computadorizada, dentre outras áreas. A propriedade interna pode ser identificada com, ou aproximada por, alguma função $f$ e o perfil pode ser identificado ou aproximado pela Transformada de Radon de $f$, designado por:

$$
\tilde{f}=\Re f
$$

Se $f$ é definida no plano $\Re^{2}$, então $\tilde{f}$ é determinada por uma integral de linha de $f$. E, se $f$ é definida em $\Re^{3}$, então $\tilde{f}$ é determinada por uma integral de superfície de $f$ sobre um plano bi-dimensional (Deans, 1993). 


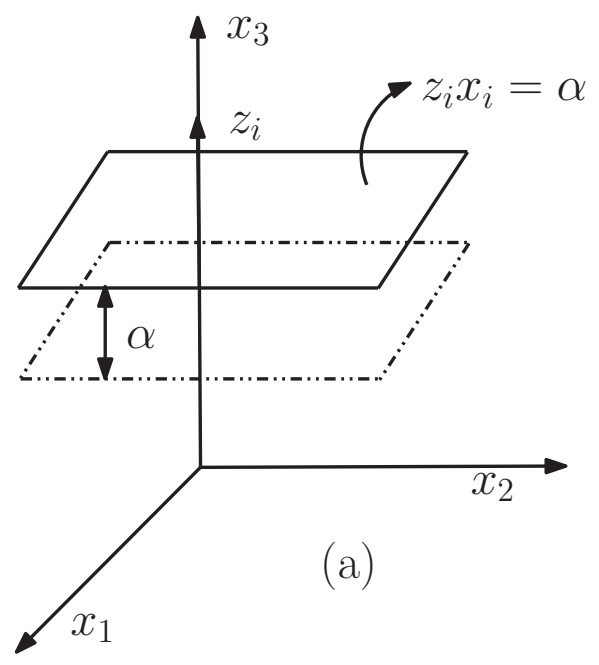

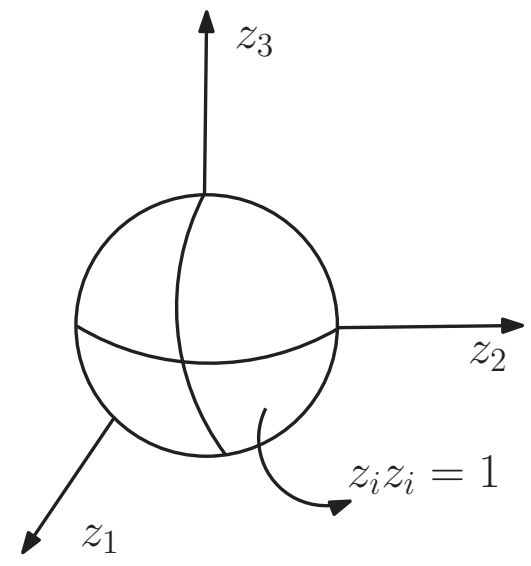

(b)

Figura B.1: Transformada de Radon para o caso tri-dimensional: integração no plano $\alpha=$ $z_{i} x_{i}$ e trasformada da Inversa: integração sobre uma esfera unitária $z_{i} z_{i}=1$.

\section{B.1 Definição da transformada de Radon}

A transformada de Radon, também conhecida como transformada de ondas planas, é descrita em detalhes por Gelfand, Graev e Vilenkin (1966). A transformada de Radon de uma função $f\left(x_{i}\right)$ é definida por (Gaul, Kögl, Wagner, 2003):

$$
\tilde{f}\left(\alpha, z_{i}\right) \equiv \Re f\left(x_{i}\right) \equiv \int_{\Omega} f\left(x_{i}\right) \delta\left(\alpha-z_{i} x_{i}\right) d \Omega
$$

onde a integração é feita sobre o plano $z_{i} x_{i}=\alpha$ (Figura B.1a) e $\delta$ representa a função delta de Dirac. A transformada inversa é dada por:

$$
f\left(x_{i}\right)=\Re^{-1} \tilde{f}\left(\alpha, z_{i}\right)=-\left.\frac{1}{8 \pi^{2}} \int_{z_{i} z_{i}=1} \frac{\partial^{2} \tilde{f}\left(\alpha, z_{i}\right)}{\partial \alpha^{2}}\right|_{\alpha=z_{i} x_{i}} d \Gamma\left(z_{i}\right)
$$

onde a integração é feita, no caso tri-dimensional, sobre uma superfície de uma esfera unitária (Figura B.1b).

A transformada de Radon possui as seguintes propriedades: 
- Homogeniedade: $\Re\left\{f\left(k \alpha, k z_{i}\right)\right\}=\frac{1}{|k|} \Re\left\{f\left(\alpha, z_{i}\right)\right\}$.

- Linearidade: $\Re\left\{c_{1} f+c_{2} g\right\}=c_{1} \Re\{f\}+c_{2} \Re\{g\}$.

- Transformação de derivadas: $\Re\left\{\frac{\partial^{n} f}{\partial x_{i} \partial x_{j} \ldots}\right\}=z_{i} z_{j} \ldots \frac{\partial^{n}}{\partial \alpha^{n}} \Re\{f\}$.

- Transformação do delta de Dirac: $\Re\left\{\delta\left(x_{i}\right)\right\}=\delta(\alpha)$.

\section{B.2 Exemplo do uso da transformada de Radon}

Para mostrar como se usa a transformada de Radon na obtenção das soluções fundamentais, considere a equação diferencial de Laplace:

$$
\frac{\partial^{2} T^{*}}{\partial x^{2}}+\frac{\partial^{2} T^{*}}{\partial y^{2}}+\frac{\partial^{2} T^{*}}{\partial z^{2}}=\delta\left(\mathbf{X}_{\mathbf{o}}\right) \quad \mathbf{X}_{\mathbf{o}}=\left(x_{o}, y_{o}, z_{o}\right)
$$

Aplicando as propriedades de transformação das derivadas e do delta de Dirac, tem-se

$$
z_{1}^{2} \frac{\partial^{2} \tilde{T}}{\partial \alpha^{2}}+z_{2}^{2} \frac{\partial^{2} \tilde{T}}{\partial \alpha^{2}}+z_{3}^{2} \frac{\partial^{2} \tilde{T}}{\partial \alpha^{2}}=\delta(\alpha)
$$

Então:

$$
\frac{\partial^{2} \tilde{T}}{\partial \alpha^{2}}=\frac{\delta(\alpha)}{z_{1}^{2}+z_{2}^{2}+z_{3}^{2}}
$$

Aplicando a transformada inversa, equação (H.16), tem-se:

$$
T^{*}(\mathbf{X})=-\frac{1}{8 \pi^{2}} \int_{z_{i} z_{i}=1} \frac{\partial^{2} \tilde{T}\left(\alpha, z_{i}\right)}{\partial \alpha^{2}} d \Gamma
$$

Usando a equação (B.6), tem-se:

$$
T^{*}(\mathbf{X})=-\frac{1}{8 \pi^{2}} \int_{z_{i} z_{i}=1} \frac{\delta(\alpha)}{z_{1}^{2}+z_{2}^{2}+z_{3}^{2}} d \Gamma
$$


considerando a origem dos eixos $x_{i}$ no ponto fonte, pode-se escrever:

$$
\alpha=z_{i} x_{i}=z_{i} r_{i}=r z_{i} r_{i}^{0}
$$

onde $r_{i}^{0}=r_{i} / r$.

Então:

$$
T^{*}(\mathbf{X})=-\frac{1}{8 \pi^{2}} \int_{z_{i} z_{i}=1} \frac{\delta\left(r_{i} z r_{i}^{0}\right)}{z_{1}^{2}+z_{2}^{2}+z_{3}^{2}} d \Gamma
$$

Usando as propriedades da transformada de Radon (homogeniedade: $\delta\left(r z r_{i}^{0}\right)=\delta\left(z_{i} r_{i}^{0}\right) / r$, e a transformada do delta de Dirac), pode-se escrever:

$$
T^{*}(\mathbf{X})=-\frac{1}{8 \pi^{2}} \int_{0}^{2 \pi} \frac{1}{z_{1}(\phi)^{2}+z_{2}(\phi)^{2}+z_{3}(\phi)^{2}} d \phi
$$

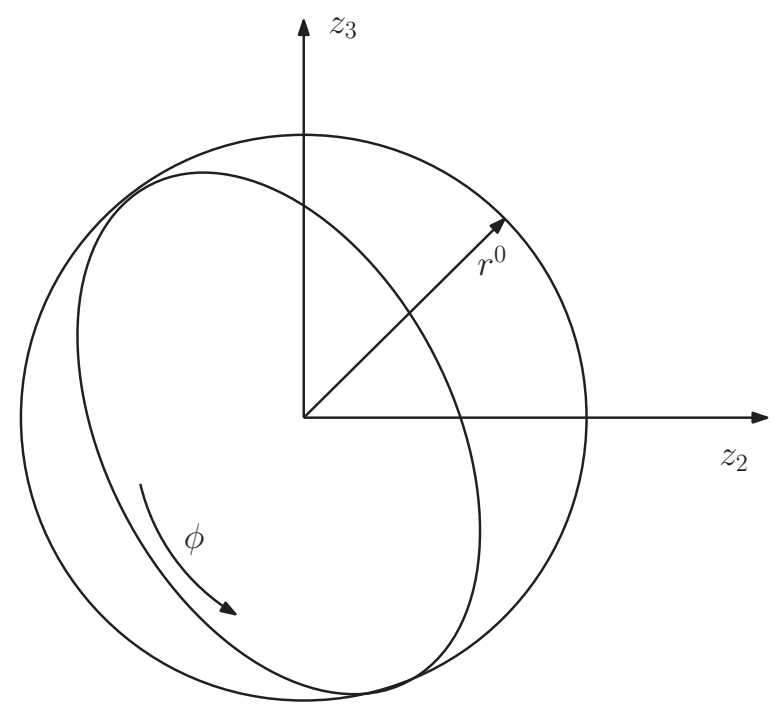

Figura B.2: Coordenadas esféricas $z_{1}, z_{2}$ e $z_{3}$.

Como $z_{1}, z_{2}$ e $z_{3}$ são coordenadas de um ponto num círculo de raio unitário (Figura B.2), $z_{1}(\phi)^{2}+z_{2}(\phi)^{2}+z_{3}(\phi)^{2}=1$. Finalmente tem-se:

$$
T^{*}(\mathbf{X})=-\left.\frac{1}{8 \pi^{2} r} \phi\right|_{0} ^{2 \pi}=-\frac{1}{8 \pi^{2} r} \times(2 \pi)=-\frac{1}{4 \pi r}
$$


que é a solução fundamental analítica para a equação de Laplace, tri-dimensional, presente na maioria dos livros do método dos elementos de contorno. 


\section{Apêndice $\mathrm{C}$}

\section{Método das diferenças finitas}

O método das diferenças finitas (MDF) é um método simples usado para resolução de problemas numéricos. O método de diferenças finitas baseia-se na aproximação de derivadas por diferenças finitas. O principio baseia-se nos primeiros termos da série de Taylor. A primeira e segunda derivada de uma função $F(x, y)$ pelas respectivas equações de diferenças dividas de primeira e de segunda ordem em $x$ e $y$. Diferenças central:

$$
\begin{aligned}
& \frac{d F}{d x}=\frac{F\left(x_{i+1}, y_{j}\right)-F\left(x_{i-1}, y_{j}\right)}{x_{i+1}-x_{i-1}}=\frac{F_{i+1, j}-F_{i-1, j}}{2 h} \\
& \frac{d F}{d y}=\frac{F\left(x_{i}, y_{j+1}\right)-F\left(x_{i}, y_{j-1}\right)}{y_{i+1}-y_{i-1}}=\frac{F_{i, j+1}-F_{i, j-1}}{2 l}
\end{aligned}
$$

Diferença de segunda ordem:

$$
\begin{aligned}
& \frac{d^{2} F}{d x^{2}}=\frac{F\left(x_{i-1}, y_{j}\right)-2 F\left(x_{i}, y_{j}\right)+F\left(x_{i+1}, y_{j}\right)}{\left(x_{i+1}-x_{i-1}\right)^{2}}=\frac{F_{i-1, j}-2 F_{i, j}+F_{i+1, j}}{h^{2}} \\
& \frac{d^{2} F}{d y^{2}}=\frac{F\left(y_{i}, y_{j-1}\right)-2 F\left(x_{i}, y_{j}\right)+F\left(x_{i}, y_{j+1}\right)}{\left(y_{i+1}-y_{i-1}\right)^{2}}=\frac{F_{i, j-1}-2 F_{i, j}+F_{i, j+1}}{l^{2}}
\end{aligned}
$$

onde $h$ e $l$ representam os incrementos nas direções $x$ e $y$, respectivamente. 


\section{Apêndice D}

\section{Funções singulares}

Integração numérica é um dos procedimentos numéricos encontrados com grande frequência na solução de equações diferencias sem solução exata. Quando funções suaves precisam ser integradas, técnicas clássicas tendem a ser adequadas, especialmente em uma e duas dimensões. Um das ferramentas principais no uso da integração numéricas é a quadratura de Gauss. Nos casos onde é necessário uma quadratura extremamente eficiente são necessários a quadratura de Gauss generalizada. O uso da quadratura Gaussiana depende da escolha do problema. Quando as funções singulares são integradas, a situação tende a ser menos satisfatória. As quadraturas Gaussianas podem ser facilmente construídas para funções da forma:

$$
f(x)=s(x) \cdot \varphi(x)
$$

onde $s$ é uma função singular e $\alpha$ é uma função suave. Entretando, tal situação é relativamente rara. Mais frequentemente, são encontradas funções com integrandos da seguinte forma:

$$
f(x)=s(x) \cdot \varphi(x)+\psi(x)
$$

onde $s$ é uma função singular fixa, e $\varphi$ e $\psi$ são funções suaves distintas (geralmente, diferentes funções singulares são envolvidas). Neste caso, a quadratura Gaussiana não pode ser usada 
diretamente, e mais especificamente nos últimos anos, as quadraturas de Gauss generalizadas foram desenvolvidas como ferramenta para lidar com tais situações. A situação é mais complicada quando (como acontece frequentemente nos kernels da solução fundamental de placas) as integrais a serem avaliadas não são estritamente falando "integrais" mas envolvem expressões da forma:

$$
\begin{aligned}
& \int_{-1}^{1} \frac{\varphi(x)}{(y-x)} d x \\
& \int_{-1}^{1} \frac{\varphi(x)}{(y-x)^{2}} d x \\
& \int_{-1}^{1} \frac{\varphi(x)}{(y-x)^{3}} d x
\end{aligned}
$$

etc., na engenharia as integrais das equações (D.3) à (D.5) são geralmente conhecidas como integrais singulares. As integrais das equações (D.3) à (D.5) são tratadas através de técnicas especiais de integração numérica. Normalmente, as singularidades são tratadas usando diferentes abordagens de forma que cada uma pode ser tratada por um processo adequado. Por exemplo, na equação (D.1) poderá ser integrada usando uma quadratura adequada para tratar a função $\varphi(x)$ que pode ser do tipo $\log (x), 1 / x$ e $1 / x^{2}$. Na equação (D.2) as funções $\varphi(x)$ e $\psi(x)$ podem ser do tipo $\log (x), 1 / x$ e $1 / x^{2}$, e serão tratadas de forma individual para cada quadratura adequada e em seguida somadas. Neste trabalho, foram desenvolvidas quadraturas numéricas para integrandos que são combinações algébricas de funções suaves e funções com singularidades da forma $\log (x), 1 / x$ e $1 / x^{2}$. 


\section{Apêndice E}

\section{Transformada de Telles}

Telles (1987) apresentou um método eficiente para calcular integrais fracamente singulares ou quase-singulares que atualmente são encontradas em aplicações do método dos elementos de contorno em problemas de duas dimensões, axissimétricos e três-dimensões. Trata-se de uma transformação polinomial de terceiro grau que melhora a aproximação do método da quadratura de Gauss dentro da faixa perto da singularidade. Este procedimento pode ser facilmente implementado no método dos elementos de contorno e apresenta uma importante característica de ser auto-adaptável, isto é, o método produz uma variável que depende da miníma distância do ponto fonte ao elemento. A auto-adaptação do método também o torna inativo quando não for útil, ou seja, em grandes distâncias do ponto fonte, o que faz o método muito seguro para o uso em geral.

Considere a integral:

$$
I=\int_{-1}^{1} f(\eta) d \eta
$$

a qual $f(\eta)$ é singular no ponto $\bar{\eta}$.

A idéia agora é fazer uma transformação de coordenadas de $\eta$ para $\gamma$ onde o jacobiano $\frac{d \eta}{d \gamma}$ se anule no ponto $\bar{\eta}$ onde $f(\eta)$ é singular. 
Para o seguinte trabalho foi escolhido uma relação de terceiro grau da seguinte forma:

$$
\eta(\gamma)=a \gamma^{3}+b \gamma^{2}+c \gamma+d
$$

As seguintes condições tem que ser satisfeitas:

$$
\begin{aligned}
\left.\frac{d^{2} \eta}{d \gamma^{2}}\right|_{\bar{\eta}} & =0 \\
\left.\frac{d \eta}{d \gamma}\right|_{\bar{\eta}} & =0 \\
\eta(1) & =1 \\
\eta(-1) & =-1
\end{aligned}
$$

Assim, a expressão (H.7) pode ser escrita como:

$$
I=\int_{-1}^{1} f\left[(\gamma-\bar{\gamma})^{3}+\bar{\gamma}\left(\bar{\gamma}^{2}+3\right)\right] /\left(1+3 \bar{\gamma}^{2}\right) 3\left(\gamma-\bar{\gamma}^{2}\right) /\left(1+3 \bar{\gamma}^{2}\right) d \gamma
$$

onde $\bar{\gamma}$ é o valor de $\gamma$ que satisfaz $\eta(\bar{\gamma})=\bar{\eta}$, este parâmetro pode ser calculado por:

$$
\bar{\gamma}=\sqrt[3]{\left(\bar{\gamma} \gamma^{*}+\left|\gamma^{*}\right|\right)}+\sqrt[3]{\left(\bar{\gamma} \gamma^{*}-\left|\gamma^{*}\right|\right)}+\gamma
$$

onde $\gamma^{*}=\bar{\gamma}^{2}-1$.

A transformação acima pode ser usada para calcular integrais com uma singularidade logaritímica em uma das extremidades. A principal vantagem é que, desde que o jacobiano cancele a singularidade, a integração de Gauss padrão pode ser aplicada sem precisar separar a parte regular da singular.

Na Figura E.1, a transformada de Telles muda a posição dos pontos de Gauss, aproximando estes pontos perto da singularidade e afastando-os na região longe da singularidade. 


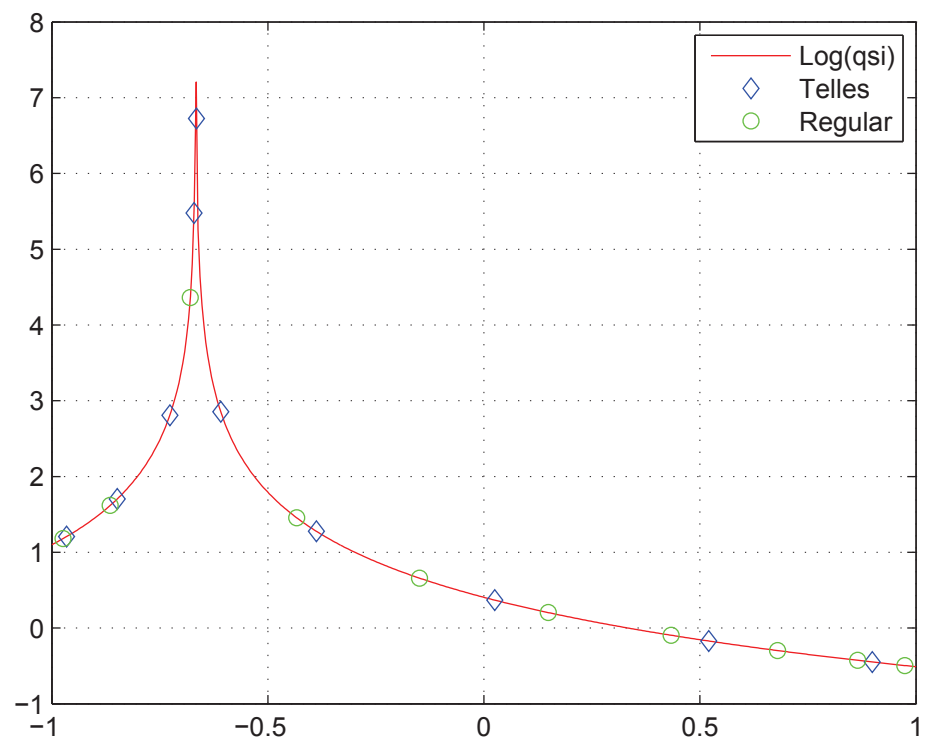

Figura E.1: Pontos de Gauss sem o uso da transformada de Telles (regular) e com seu uso. 


\section{Apêndice F}

\section{Quadratura para integrais singulares}

Integração numérica é um dos mais recorrentes procedimentos encontrados em métodos numéricos. Quando o integrando é polinomial ou pelo menos suave, a quadratura clássica de Gauss é extremamente eficiente. Por outro lado, quando as funções a ser integradas apresentam pontos singulares ao longo do seu domínio, este procedimento é pouco eficiente, necessitando de muitos pontos para se obter um resultado com precisão adequada, ou mesmo inapropriado, quando a função apresenta singularidades fortes e hipersingularidades.

\section{F.1 Definição}

Equações que envolvem integrais do tipo:

$$
\int_{a}^{b} \frac{f(x)}{(x-c)} d x
$$

onde $a<c<b$, não é integrável no sentido comum sobre qualquer intervalo que inclui o ponto $x=c$. Neste caso, a integral pode ser regularizada pelo valor principal de Cauchy (VPC) da seguinte forma: 


$$
\text { p.v. } \int_{a}^{b} \frac{f(x)}{(x-c)} d x:=\lim _{\epsilon \rightarrow 0}\left(\int_{a}^{c-\epsilon} \frac{f(x)}{(x-c)} d x+\int_{c+\epsilon}^{b} \frac{f(x)}{(x-c)} d x\right)
$$

Para cancelar a singularidade no VPC, a função $f(x)$ precisa ser contínua em $(a, b)$ para que em torno do ponto singular $x=c$ o integrando possa ser simétrico. O VPC pode também ser avaliado em separado de ambos os lados da seguinte forma:

$$
\begin{gathered}
\text { p.v. } \int_{a}^{c} \frac{f(x)}{(x-c)} d x=\lim _{\epsilon \rightarrow 0}\left(\int_{a}^{c-\epsilon} \frac{f(x)}{(x-c)} d x-f(c) \ln (\epsilon)\right) \\
\text { p.v. } \int_{c}^{b} \frac{f(x)}{(x-c)} d x=\lim _{\epsilon \rightarrow 0}\left(\int_{c+\epsilon}^{b} \frac{f(x)}{(x-c)} d x+f(c) \ln (\epsilon)\right)
\end{gathered}
$$

Para integrais com singularidades de ordem superior, o VPC não existe. Se o valor principal de Cauchy (VPC) é diferenciado em relação a $x$ é dado por:

$$
\frac{d}{d x} \int_{a}^{b} \frac{f(x)}{(x-c)} d x=\int_{a}^{b} \frac{f(x)}{(x-c)^{2}} d x
$$

Esta equação (F.4) é chamada de integral hipersingular ou valor principal de Hadamard (VPH). Para esta integral há também outras nomenclaturas, como:

$$
\int_{a}^{b} \frac{f(x)}{(x-c)^{2}} d x=\frac{d}{d x} \int_{a}^{b} \frac{f(x)}{(x-c)} d x
$$

Há tambem outra definição, como:

$$
\int_{a}^{b} \frac{f(x)}{(x-c)^{2}} d x=\lim _{\epsilon \rightarrow 0}\left[\int_{a}^{c-\epsilon} \frac{f(x)}{(x-c)^{2}} d x+\int_{c+\epsilon}^{b} \frac{f(x)}{(x-c)^{2}} d x-\frac{2 f(x)}{\epsilon}\right]
$$

Outras informações sobre integrais hipersingulares podem ser encontradas em Gel'fand e Shilov (1967). 


\section{F.2 Quadratura proposta}

Considere agora a substituição da equação (F.4) por uma quadratura de ordem $n$ (Campos e Albuquerque, 2013):

$$
f . p . \int_{a}^{b} \frac{f(x)}{(x-c)^{2}} d x=\sum_{i=1}^{n} w_{i} f\left(x_{i}\right)
$$

A quadratura proposta é capaz de avaliar exatamente a integral quando $f(x)$ é um polinômio de um grau pré determinado $n$. Quando $f(x)$ é uma função diferente de um polinômio ela vai ser aproximada por um polinômio, do mesmo modo que a clássica quadratura de Gauss. Bons resultados são esperados quando $f(x)$ é suave. Para obter os pontos e os pesos da quadratura, um sistema não-linear de equações é proposto na seguinte forma:

$$
\begin{aligned}
& \sum_{i=1}^{n} w_{i} P_{0}\left(x_{i}\right)=f \cdot p \cdot \int_{a}^{b} \frac{P_{0}(x)}{(x-c)^{2}} \\
& \sum_{i=1}^{n} w_{i} P_{1}\left(x_{i}\right)=f \cdot p \cdot \int_{a}^{b} \frac{P_{1}(x)}{(x-c)^{2}} \\
& \sum_{i=1}^{n} w_{i} P_{2}\left(x_{i}\right)=f \cdot p \cdot \int_{a}^{b} \frac{P_{2}(x)}{(x-c)^{2}} \\
& \vdots \\
& \sum_{i=1}^{n} w_{i} P_{2 n-1}\left(x_{i}\right)=f \cdot p \cdot \int_{a}^{b} \frac{P_{2 n-1}(x)}{(x-c)^{2}}
\end{aligned}
$$

onde $P_{j}(x)$ são polinômios de grau $j$. Pode-se também utilizar uma regra de quadratura com pontos previamente escolhidos (as coordenadas dos pontos de integração deixam de ser variáveis desconhecidas no sistema não-linear) que irão resultar em um sistema linear, que é mais simples de resolver, mas vai precisar de mais pontos para ter a mesma ordem. A quadratura proposta irá convergir para a maioria dos casos. O sistema não-linear pode ser resolvido através do método de Newton, ou um sistema linear mais simples, de baixa ordem, em que apenas os pesos são desconhecidos. Na Figura F.1 temos a função que será resolvida usando a quadratura proposta por Campos e Albuquerque (2013). 


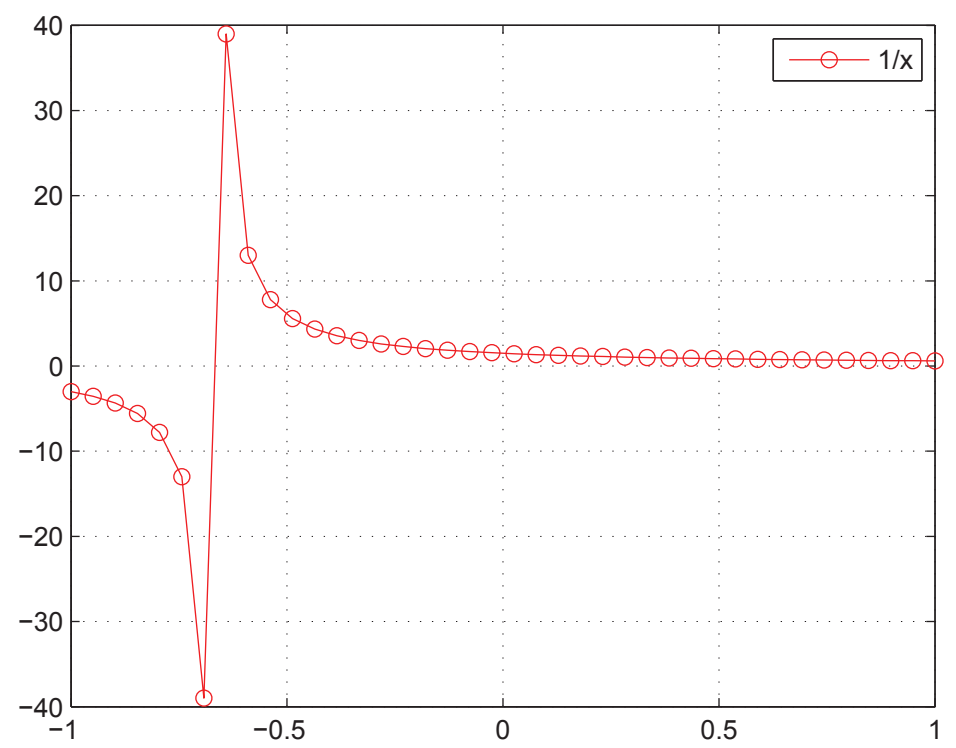

Figura F.1: Gráfico da função 1/x.

Na Tabela F.1 é mostrado a comparação entre a solução obtida através da quadratura empregada e a solução obtida através do programa comercial Mathematica.

Tabela F.1: Solução para função 1/x através da quadratura proposta por Campos e Albuquerque (2013).

\begin{tabular}{|c|c|c|c|}
\hline \multirow{2}{*}{$n$} & \multicolumn{3}{|c|}{$1 / x$} \\
\cline { 2 - 4 } & Campos e Albuquerque (2013) & Gauss & Gauss e Telles \\
\hline 1 & 1,6094 & 3 & $-11,04$ \\
\hline 2 & 1,6094 & 12 & $-39,83$ \\
\hline 3 & 1,6094 & $-3,42$ & $-25,29$ \\
\hline 4 & 1,6094 & 1,08 & $-56,38$ \\
\hline 20 & 1,6094 & 4,49 & 16,98 \\
\hline Mathematica & \multicolumn{3}{|c}{1,6094} \\
\hline
\end{tabular}

Pode-se notar na Tabela F.1 que foi necessário apenas 1 ponto de integração para resolver a integração numérica. 


\section{Apêndice G}

\section{Desenvolvimento da derivada da}

\section{solução fundamental}

Para o cálculo dos momentos foram usadas as relações de esforços generalizados dados pelas equações (G.1) e (G.2). Nas equações (G.1) e (G.2) foi inserido um ponto fonte $(\delta)$ da seguinte forma:

$$
\begin{gathered}
M_{\delta \alpha \beta}=D_{\alpha \beta}\left(\psi_{\delta \alpha, \beta}+\psi_{\delta \beta, \alpha}\right)+C_{\alpha \beta} \psi_{\delta \gamma, \gamma} \\
Q_{\delta \alpha}=C v_{\alpha}\left(W_{\delta 3, \alpha}+\psi_{\delta \alpha}\right)
\end{gathered}
$$

onde $\psi$ representa as rotações com índices 1 e 2, e $W$ representa o delocamento na direção transversal com índice 3.

Os kernels $\tilde{U}$ e $\tilde{P}$ da solução fundamental são derivadas em relação ao ponto fonte $(\delta)$ e tomam a seguinte forma:

$$
\frac{d \tilde{U}_{\alpha \beta}(\rho)}{d \rho}=a_{\alpha \beta} \frac{d^{5} \varphi}{d \rho^{5}}-C_{1} C_{2} \omega_{\alpha} \omega_{\beta} \frac{d^{3} \varphi}{d \rho^{3}}
$$




$$
\begin{gathered}
\frac{d \tilde{U}_{3 \alpha}(\rho)}{d \rho}=-\frac{\tilde{U}_{\alpha 3}(\rho)}{d \rho}=f_{\alpha} \frac{d^{4} \varphi}{d \rho^{4}}-C_{1} C_{2} \omega_{\alpha} \frac{d \varphi^{2}}{d \rho^{2}} \\
\frac{d \tilde{U}_{33}(\rho)}{d \rho}=\alpha_{1} \frac{d^{4} \varphi}{d \rho^{4}}-\beta_{1} \frac{d^{2} \varphi}{d \rho^{2}} C_{1} C_{2} \varphi \\
\left.\frac{d \tilde{P}_{\alpha \beta}(\rho)}{d \rho}=\left[D_{\beta \gamma}\left(a_{\alpha \beta} \omega_{\gamma}+a_{\alpha \beta} \omega_{\beta}\right) n_{\gamma}+C_{\beta \gamma} d_{\alpha} n_{\gamma}\right] \frac{d^{6} \varphi}{d \rho^{6}}-\left[2 D_{\beta \gamma} C_{1} C_{2} \omega_{\alpha} \omega_{\beta} \omega_{\gamma}\right) n_{\gamma}+C_{1} C_{2} C_{\beta \gamma} \omega_{\alpha} n_{\gamma}\right] \frac{d^{4} \varphi}{d \rho^{4}} \\
\frac{d \tilde{P}_{3 \alpha}(\rho)}{d \rho}=\left[D_{\alpha \gamma}\left(f_{\alpha} \omega_{\gamma}+f_{\gamma} \omega_{\alpha}\right)+C_{\alpha \gamma} g\right] n_{\gamma} \frac{d^{5} \varphi}{d \rho^{5}}-\left[2 D_{\alpha \gamma} \omega_{\alpha} \omega_{\gamma}+C_{\alpha \gamma}\right] n_{\gamma} C_{1} C_{2} \frac{d^{3} \varphi}{d \rho^{3}} \\
\frac{d \tilde{P}_{\alpha 3}(\rho)}{d \rho}=C_{\gamma}\left(a_{\alpha \gamma}-f_{\alpha} \omega_{\gamma}\right) n_{\gamma} \frac{d^{5} \varphi}{d \rho^{5}} \\
\frac{d \tilde{P}_{33}(\rho)}{d \rho}=C_{\gamma}\left(f_{\gamma}-\beta_{1} \omega_{\gamma}\right) n_{\gamma} \frac{d^{4} \varphi}{d \rho^{4}}+\alpha_{1} C_{\gamma} \omega_{\gamma} n_{\gamma} \frac{d^{6} \varphi}{d \rho^{6}}
\end{gathered}
$$

Através do uso da propriedade de Radon, muda-se o domínio de integração de $(\rho)$ para $(x, y)$, através da seguinte relação:

$$
\frac{\partial \psi}{\partial x_{\alpha}}=\omega_{\alpha} \frac{d \psi}{d \rho}
$$

onde $\omega_{1}=\cos (\theta)$ e $\omega_{2}=\sin (\theta)$. Os valores de $\psi$ e $W$ assumem os valores de $\tilde{U}$ ou $\tilde{P}$, $\operatorname{logo}$ temos as equações (G.1) e (G.2) tomam a seguinte forma:

$$
\begin{gathered}
M_{111}=2 D_{11}\left(\frac{d \psi_{11}}{d x}\right)+C_{11}\left(\frac{d \psi_{11}}{d x}+\frac{d \psi_{12}}{d y}\right) \\
M_{112}=D_{12}\left(\frac{d \psi_{11}}{d y}+\frac{d \psi_{12}}{d x}\right)+C_{12}\left(\frac{d \psi_{11}}{d x}+\frac{d \psi_{12}}{d y}\right)
\end{gathered}
$$




$$
\begin{gathered}
M_{122}=2 D_{22}\left(\frac{d \psi_{12}}{d y}\right)+C_{22}\left(\frac{d \psi_{11}}{d x}+\frac{d \psi_{12}}{d y}\right) \\
M_{121}=D_{21}\left(\frac{d \psi_{12}}{d x}+\frac{d \psi_{11}}{d y}\right)+C_{21}\left(\frac{d \psi_{11}}{d x}+\frac{d \psi_{12}}{d y}\right) \\
M_{222}=2 D_{22}\left(\frac{d \psi_{22}}{d y}\right)+C_{22}\left(\frac{d \psi_{21}}{d x}+\frac{d \psi_{22}}{d y}\right) \\
M_{221}=D_{21}\left(\frac{d \psi_{22}}{d x}+\frac{d \psi_{21}}{d y}\right)+C_{21}\left(\frac{d \psi_{21}}{d x}+\frac{d \psi_{22}}{d y}\right) \\
M_{211}=2 D_{11}\left(\frac{d \psi_{21}}{d x}\right)+C_{11}\left(\frac{d \psi_{21}}{d x}+\frac{d \psi_{22}}{d y}\right) \\
M_{311}=2 D_{11}\left(\frac{d \psi_{31}}{d x}\right)+C_{11}\left(\frac{d \psi_{31}}{d x}+\frac{d \psi_{32}}{d y}\right) \\
M_{212}=D_{12}\left(\frac{d \psi_{22}}{d x}+\frac{d \psi_{32}}{d y}\right)+C_{12}\left(\frac{d \psi_{21}}{d x}+\frac{d \psi_{22}}{d y}\right)
\end{gathered}
$$




$$
\begin{aligned}
& Q_{11}=C v_{1}\left(\frac{d \psi_{13}}{d x}+W_{11}\right) \\
& Q_{12}=C v_{2}\left(\frac{d \psi_{13}}{d y}+W_{12}\right) \\
& Q_{21}=C v_{1}\left(\frac{d \psi_{23}}{d x}+W_{21}\right) \\
& Q_{22}=C v_{2}\left(\frac{d \psi_{23}}{d x}+W_{22}\right) \\
& Q_{31}=C v_{1}\left(\frac{d \psi_{33}}{d x}+W_{31}\right) \\
& Q_{32}=C v_{2}\left(\frac{d \psi_{33}}{d x}+W_{32}\right)
\end{aligned}
$$

Os valores de $\psi$ e $W$ assumem os valores de $\tilde{U}$ ou $\tilde{P}$, respectivamente. Então, o cálculo dos momentos e esforços cortantes em pontos internos são realizados através das equações (G.29) e (G.30):

$$
\begin{array}{r}
M_{\alpha \beta}\left(X^{\prime}\right)=\int_{\Gamma} U_{\alpha \beta k}^{*}\left(X^{\prime}, x\right) p_{k}(x) d \Gamma(x)-\int_{\Gamma} P_{\alpha \beta k}^{*}\left(X^{\prime}, x\right) u_{k}(x) d \Gamma(x) \\
+q \int_{\Gamma} W_{\alpha \beta}^{*}\left(X^{\prime}, x\right) d \Gamma(x) \\
Q_{\beta}\left(X^{\prime}\right)=\int_{\Gamma} U_{3 \beta k}^{*}\left(X^{\prime}, x\right) p_{k}(x) d \Gamma(x)-\int_{\Gamma} P_{3 \beta k}^{*}\left(X^{\prime}, x\right) u_{k}(x) d \Gamma(x) \\
+q \int_{\Gamma} W_{3 \beta}^{*}\left(X^{\prime}, x\right) d \Gamma(x)
\end{array}
$$

O cálculo dos momentos e esforços cortantes no contorno são realizados através das equações (G.31) e (G.32): 


$$
\begin{array}{r}
\frac{1}{2} M_{\alpha \beta}\left(x^{\prime}\right)+\int_{\Gamma} P_{\alpha \beta \gamma}^{*}\left(x^{\prime}, x\right) u_{\gamma}(x) d \Gamma(x)+\int_{\Gamma} P_{\alpha \beta 3}^{*}\left(x^{\prime}, x\right) u_{3}(x) d \Gamma(x) \\
=\int_{\Gamma} U_{\alpha \beta \gamma}^{*}\left(x^{\prime}, x\right) p_{\gamma}(x) d \Gamma(x)+\int_{\Gamma} U_{\alpha \beta 3}^{*}\left(x^{\prime}, x\right) p_{3}(x) d \Gamma(x) \\
+q \int_{\Gamma} W_{\alpha \beta}^{*}\left(x^{\prime}, x\right) d \Gamma(x)+\frac{1}{2} \frac{q \nu}{(1-\nu) \lambda^{2}} \delta_{\alpha \beta}
\end{array}
$$

O cálculo das tensões em pontos internos ou contorno são realizados através das equações (G.33), (G.34) e (G.35):

$$
\begin{gathered}
\sigma_{\alpha \beta}=\frac{12 x_{3}}{h^{3}} M_{\alpha \beta} \\
\sigma_{3 \alpha}=\frac{3}{2 h}\left[1-\left(\frac{2 x_{3}}{h}\right)^{2}\right] Q_{\alpha} \\
\sigma_{33}=\frac{1}{4} \frac{2 x_{3}}{h}\left[3-\left(\frac{2 x_{3}}{h}\right)^{2}\right] q
\end{gathered}
$$




\section{Apêndice $H$}

\section{Desenvolvimento dos kernels da}

\section{solução fundamental $U_{i j}^{*}$}

Considere a equação diferencial de equilíbrio de placas ortotrópicas:

$$
\Delta_{i j}^{*} U_{j}+b_{i}=0
$$

onde $b_{i}$ representam 0,0 e $q$, respectivamente, e $\Delta_{i j}^{*}$ são operadores diferenciais que podem ser escritos na forma matricial como:

$$
\Delta_{i j}^{*}=\left[\begin{array}{ccc}
D_{1} \frac{\partial^{2}}{\partial x_{1}^{2}}+D_{k} \frac{\partial^{2}}{\partial x_{2}^{2}}-C_{1} & \left(D_{1} \mu_{y x}+D_{k}\right) \frac{\partial^{2}}{\partial x_{1} \partial x_{2}} & -C_{1} \frac{\partial}{\partial x_{1}} \\
\left(D_{1} \mu_{y x}+D_{k}\right) \frac{\partial^{2}}{\partial x_{1} \partial x_{2}} & D_{1} \frac{\partial^{2}}{\partial x_{1}^{2}}+D_{k} \frac{\partial^{2}}{\partial x_{2}^{2}}-C_{2} & -C_{2} \frac{\partial}{\partial x_{2}} \\
-C_{1} \frac{\partial}{\partial x_{1}} & -C_{2} \frac{\partial}{\partial x_{2}} & C_{1} \frac{\partial^{2}}{\partial x_{1}^{2}}+C_{2} \frac{\partial^{2}}{\partial x_{2}^{2}}
\end{array}\right]
$$

Os cofatores da matriz $\Delta^{*}$ são dados em notação indicial por:

$$
{ }^{c o} \Delta_{\alpha \beta}^{*}=E_{\alpha \beta} \nabla^{2} \nabla_{k}^{2}-B_{\alpha \beta} \frac{\partial^{2}}{\partial x_{\alpha} \partial x_{\beta}} \nabla_{k}^{2}-C_{1} C_{2} \frac{\partial^{2}}{\partial x_{\alpha} \partial x_{\beta}}
$$




$$
\begin{gathered}
{ }^{c o} \Delta_{3 \alpha}^{*}=-{ }^{c o} \Delta_{\alpha 3}^{*}=\frac{\partial}{\partial x_{\alpha}}\left(E_{\alpha 3} \frac{\partial^{2}}{\partial x_{2}^{2}}+B_{\alpha 3} \frac{\partial^{2}}{\partial x_{1}^{2}}-C_{1} C_{2}\right) \\
{ }^{c o} \Delta_{33}^{*}=D_{1} D_{k} \frac{\partial^{4}}{\partial x_{1}^{4}}+\left(D_{1} D_{2}-D_{1}^{2} \mu_{y x}^{2}-2 D_{1} D_{k} \mu_{y x}\right) \frac{\partial^{4}}{\partial x_{1}^{2} \partial x_{2}^{2}} \\
+D_{2} D_{k} \frac{\partial^{4}}{\partial x_{2}^{4}}-\left(D_{1} C_{2}+C_{1} D_{k}\right) \frac{\partial^{2}}{\partial x_{1}^{2}}-\left(C_{1} D_{2}+C_{2} D_{k}\right) \frac{\partial^{2}}{\partial x_{2}^{2}}+C_{1} C_{2}
\end{gathered}
$$

Então, a matriz de cofatores pode ser escrita como:

$$
{ }^{c o} \Delta^{a d j}=\left[\begin{array}{cc}
{ }^{c o} \Delta_{\alpha \beta}^{a d j} & { }^{c o} \Delta_{\alpha 3}^{a d j} \\
{ }^{c o} \Delta_{3 \beta}^{a d j} & { }^{c o} \Delta_{33}^{a d j}
\end{array}\right]
$$

A transposta da matriz de cofatores é dada por:

$$
\left({ }^{c o} \Delta^{a d j}\right)^{T}=\left[\begin{array}{cc}
{ }^{c o} \Delta_{\alpha \beta}^{a d j} & { }^{c o} \Delta_{3 \alpha}^{a d j} \\
{ }^{c o} \Delta_{\alpha 3}^{a d j} & { }^{c o} \Delta_{33}^{a d j}
\end{array}\right]
$$

Em seguida, calcula-se o determinante da matriz transposta:

$$
\operatorname{det}\left|\left({ }^{c o} \Delta_{i j}^{a d j}\right)^{T}\right|={ }^{c o} \Delta_{\alpha \beta}^{a d j} \cdot{ }^{c o} \Delta_{33}^{a d j}-{ }^{c o} \Delta_{3 \alpha}^{a d j} \cdot{ }^{c o} \Delta_{\alpha 3}^{a d j}
$$

De acordo com Hörmander (ver Apêndice A), precisa-se obter um escalar $\Phi$ que satisfaça a seguinte equação:

$$
\operatorname{det}\left|\left({ }^{c o} \Delta_{i j}^{a d j}\right)^{T}\right| \Phi(\xi, x)=-\delta(\xi, x)
$$

A função escalar $\Phi$ é dada por:

$$
\Phi(\zeta, x)=\int_{0}^{2 \pi} \varphi(\rho) d \theta
$$


onde

$$
\rho=\omega_{1}(x-\xi)+\omega_{2}(y-\eta)
$$

O cálculo do kernel $\tilde{U}_{i j}$ da solução fundamental $U_{i j}^{*}$ é dado por:

$$
\tilde{U}_{i j}={ }^{c o} \Delta_{i j}^{a d j} \Phi
$$

e

$$
U_{i j}^{*}=\int_{0}^{2 \pi} \tilde{U}_{i j}(\rho) d \theta
$$

\section{H.1 Desenvolvimento do kernel $\tilde{U}_{\alpha \beta}$}

Considere o operador diferencial dado pela equação (H.3):

$$
{ }^{c o} \Delta_{\alpha \beta}=E_{\alpha \beta} \nabla^{2} \nabla_{k}^{2}-B_{\alpha \beta} \frac{\partial^{2}}{\partial x_{\alpha} \partial x_{\beta}} \nabla_{k}^{2}-C_{1} C_{2} \frac{\partial^{2}}{\partial x_{\alpha} \partial x_{\beta}}
$$

Abrindo os termos $\nabla^{2}$ e $\nabla_{k}^{2}$ presentes na equação (H.13) e usando a relação dada pela equação (H.14), obtém-se:

$$
\begin{gathered}
\frac{\partial}{\partial x_{\alpha}}=\omega_{\alpha} \frac{d}{d \rho} \\
\nabla^{2}=\frac{\partial^{2}}{\partial x_{1}^{2}}+\frac{\partial^{2}}{\partial x_{1}^{2}}=\omega_{1}^{2} \frac{d^{2}}{d \rho^{2}}+\omega_{2}^{2} \frac{d^{2}}{d \rho^{2}} \\
\nabla_{k}^{2}=C_{1} \frac{\partial^{2}}{\partial x_{1}^{2}}+C_{2} \frac{\partial^{2}}{\partial x_{1}^{2}}=C_{1} \omega_{1}^{2} \frac{d^{2}}{d \rho^{2}}+C_{2} \omega_{2}^{2} \frac{d^{2}}{d \rho^{2}}
\end{gathered}
$$

Substituindo as equações (H.15) e (H.16) na equação (H.13), obtém-se: 


$$
\begin{gathered}
{ }^{c o} \Delta_{\alpha \beta}=E_{\alpha \beta}\left(\omega_{1}^{2} \frac{d^{2}}{d \rho^{2}}+\omega_{2}^{2} \frac{d^{2}}{d \rho^{2}}\right) \cdot\left(C_{1} \omega_{1}^{2} \frac{d^{2}}{d \rho^{2}}+C_{2} \omega_{2}^{2} \frac{d^{2}}{d \rho^{2}}\right) \\
-B_{\alpha \beta} \omega_{1} \omega_{2} \frac{d^{2}}{d \rho^{2}}\left(C_{1} \omega_{1}^{2} \frac{d^{2}}{d \rho^{2}}+C_{2} \omega_{2}^{2} \frac{d^{2}}{d \rho^{2}}\right)-C_{1} C_{2} \omega_{1}^{2} \omega_{2}^{2} \frac{d^{2}}{d \rho^{2}} \\
{ }^{c o} \Delta_{\alpha \beta}=E_{\alpha \beta} \frac{d^{2}}{d \rho^{2}}\left(\omega_{1}^{2}+\omega_{2}^{2}\right) \frac{d^{2}}{d \rho^{2}}\left(C_{1} \omega_{1}^{2}+C_{2} \omega_{2}^{2}\right) \\
-B_{\alpha \beta} \omega_{1} \omega_{2} \frac{d^{2}}{d \rho^{2}} \frac{d^{2}}{d \rho^{2}}\left(C_{1} \omega_{1}^{2}+C_{2} \omega_{2}^{2}\right) C_{1} C_{2} \omega_{1}^{2} \omega_{2}^{2} \frac{d^{2}}{d \rho^{2}} \\
{ }^{c o} \Delta_{\alpha \beta}=E_{\alpha \beta}\left(\omega_{1}^{2}+\omega_{2}^{2}\right)\left(C_{1} \omega_{1}^{2}+C_{2} \omega_{2}^{2}\right) \frac{d^{4}}{d \rho^{4}} \\
-B_{\alpha \beta} \omega_{1} \omega_{2}\left(C_{1} \omega_{1}^{2}+C_{2} \omega_{2}\right) \frac{d^{4}}{d \rho^{4}}-C_{1} C_{2} \omega_{1}^{2} \omega_{2}^{2} \frac{d^{2}}{d \rho^{2}}
\end{gathered}
$$

Após algumas simplificações chega-se a equação (H.17):

$$
\begin{array}{r}
{ }^{c o} \Delta_{\alpha \beta}=\left[E_{\alpha \beta}\left(\omega_{1}^{2}+\omega_{2}^{2}\right)\left(C_{1} \omega_{1}^{2}+C_{2} \omega_{2}^{2}\right)\right. \\
\left.-B_{\alpha \beta} \omega_{1} \omega_{2}\left(C_{1} \omega_{1}^{2}+C_{2} \omega_{2}^{2}\right)\right] \frac{d^{4}}{d \rho^{4}}-C_{1} C_{2} \omega_{1}^{2} \omega_{2}^{2} \frac{d^{2}}{d \rho^{2}}
\end{array}
$$

Usando da identidade trigonométrica $\omega_{1}^{2}+\omega_{2}^{2}$ pode-se simplicar ainda mais a equação (H.17), ou seja, lembrando que $\omega_{1}=\sin (\theta)$ e $\omega_{2}=\cos (\theta)$, tem-se:

$$
\sin ^{2}(\theta)+\cos ^{2}(\theta)=1
$$

Dessa forma, tem-se:

$$
{ }^{c o} \Delta_{\alpha \beta}=\left(E_{\alpha \beta}-B_{\alpha \beta} \omega_{1} \omega_{2}\right)\left(C_{1} \omega_{1}^{2}+C_{2} \omega_{2}^{2}\right) \frac{d^{4}}{d \rho^{4}}-C_{1} C_{2} \omega_{1}^{2} \omega_{2}^{2} \frac{d^{2}}{d \rho^{2}}
$$


Substituindo a equação (H.18) em (H.11), obtém-se o kernel $\tilde{U}_{3 \alpha}$ da solução fundamental:

$$
\tilde{U}_{\alpha \beta}^{*}=\int_{0}^{2 \pi}\left(a_{\alpha \beta} \frac{d^{4}}{d \rho^{4}}-C_{1} C_{2} \omega_{1}^{2} \omega_{2}^{2} \frac{d^{2}}{d \rho^{2}}\right) \varphi d \theta
$$

onde o termo $a_{\alpha \beta}$ é dado por:

$$
a_{\alpha \beta}=\left(E_{\alpha \beta}-B_{\alpha \beta} \omega_{\alpha} \omega_{\beta}\right)\left(C_{1} \omega_{1}^{2}+C_{2} \omega_{2}^{2}\right)
$$

\section{H.2 Desenvolvimento dos kernels $\tilde{U}_{3 \alpha}=-\tilde{U}_{\alpha 3}$}

o mesmo procedimento da subseção anterior temos o operador diferencial dado pela equação (H.20), tem-se:

$$
{ }^{c o} \Delta_{3 \alpha}^{*}=-{ }^{c o} \Delta_{\alpha 3}^{*}=\frac{\partial}{\partial x_{\alpha}}\left(E_{\alpha 3} \frac{\partial^{2}}{\partial x_{2}^{2}}+B_{\alpha 3} \frac{\partial^{2}}{\partial x_{1}^{2}}-C_{1} C_{2}\right)
$$

Fazendo o uso da equação (H.14), tem-se:

$$
\begin{gathered}
{ }^{c o} \Delta_{3 \alpha}^{*}=\omega_{\alpha} \frac{d}{d \rho}\left(E_{\alpha 3} \omega_{2}^{2} \frac{d^{2}}{d \rho^{2}}+B_{\alpha 3} \omega_{1}^{2} \frac{d^{2}}{d \rho^{2}}-C_{1} C_{2}\right) \\
{ }^{c o} \Delta_{3 \alpha}^{*}=\frac{d^{3}}{d \rho^{3}}\left(E_{\alpha 3} \omega_{2}^{2}+B_{\alpha 3} \omega_{1}^{2}\right) \omega_{\alpha}-C_{1} C_{2} \omega_{\alpha} \frac{d}{d \rho}
\end{gathered}
$$

Após algumas simplificações chega-se a equação (H.20):

$$
{ }^{c o} \Delta_{3 \alpha}^{*}=f_{\alpha} \frac{d^{3}}{d \rho^{3}}-C_{1} C_{2} \omega_{\alpha} \frac{d}{d \rho}
$$


onde o termo $f_{\alpha}$, é dado por:

$$
f_{\alpha}=\left(E_{\alpha 3} \omega_{2}^{2}+B_{\alpha 3} \omega_{1}^{2}\right) \omega_{\alpha}
$$

Assim, o kernel $\tilde{U}_{3 \alpha}$ da solução fundamental é dado por:

$$
\tilde{U}_{3 \alpha}^{*}=-\tilde{U}_{\alpha 3}^{*}=\int_{0}^{2 \pi}\left(f_{\alpha} \frac{d^{3} \varphi}{d \rho^{3}}-C_{1} C_{2} \omega_{\alpha} \frac{d \varphi}{d \rho}\right) d \theta
$$

\section{H.3 Desenvolvimento do kernel $\tilde{U}_{33}$}

Seguindo o mesmo procedimento da subseção anterior temos o operador diferencial dado pela equação (H.23), tem-se:

$$
\begin{array}{r}
{ }^{c o} \Delta_{33}^{*}=D_{1} D_{k} \frac{\partial^{4}}{\partial x_{1}^{4}}+\left(D_{1} D_{2}-D_{1}^{2} \mu_{y x}^{2}-2 D_{1} D_{k} \mu_{y x}\right) \frac{\partial^{4}}{\partial x_{1}^{2} \partial x_{2}^{2}} \\
+D_{2} D_{k} \frac{\partial^{4}}{\partial x_{2}^{4}}-\left(D_{1} C_{2}+C_{1} D_{k}\right) \frac{\partial^{2}}{\partial x_{1}^{2}}-\left(C_{1} D_{2}+C_{2} D_{k}\right) \frac{\partial^{2}}{\partial x_{2}^{2}}+C_{1} C_{2}
\end{array}
$$

Fazendo o uso da equação (H.14), tem-se:

$$
\begin{array}{r}
{ }^{c o} \Delta_{33}^{*}=D_{1} D_{k} \omega_{1}^{4} \frac{d^{4}}{d \rho^{4}}+\left(D_{1} D_{2}-D_{1}^{2} \mu_{y x}^{2}-2 D_{1} D_{k} \mu_{y x}\right) \omega_{1}^{2} \omega_{2}^{2} \frac{d^{4}}{d \rho^{4}} \\
+D_{2} D_{k} \omega_{2}^{4} \frac{d^{4}}{d \rho^{4}}-\left(D_{1} C_{2}+C_{1} D_{k}\right) \omega_{1}^{2} \frac{d^{2}}{d \rho^{2}}-\left(C_{1} D_{2}+C_{2} D_{k}\right) \omega_{2}^{2} \frac{d^{2}}{d \rho^{2}}+C_{1} C_{2}
\end{array}
$$

Após algumas simplificações chega-se a equação (H.24):

$$
{ }^{c o} \Delta_{33}^{*}=\alpha_{1} \frac{d^{4}}{d \rho^{4}}-\beta_{1} \frac{d^{2}}{d \rho^{2}}+C_{1} C_{2}
$$

onde $\alpha_{1}$ e $\beta_{1}$ são dados por: 


$$
\begin{gathered}
\alpha_{1}=D_{1} D_{k} \omega_{1}^{4}+\left(D_{1} D_{2}-D_{1}^{2} \mu_{y x}^{2}-2 D_{1} D_{k} \mu_{y x}\right) \omega_{1}^{2} \omega_{2}^{2}+D_{2} D_{k} \omega_{2}^{4} \\
\beta_{1}=\left(D_{1} C_{2}+C_{1} D_{k}\right) \omega_{1}^{2}+\left(C_{1} D_{2}+C_{2} D_{k}\right) \omega_{2}^{2}
\end{gathered}
$$

Assim, o kernel $\tilde{U}_{33}$ da solução fundamental é dado por:

$$
\tilde{U}_{33}^{*}=\int_{0}^{2 \pi}\left(\alpha_{1} \frac{d^{4} \varphi}{d \rho^{4}}-\beta_{1} \frac{d^{2} \varphi}{d \rho^{2}}+C_{1} C_{2} \varphi\right) d \theta
$$

